\title{
1,2-Selective Hydrosilylation of Conjugated Dienes
}

\section{Citation}

Parker, Sarah Elizabeth. 2014. 1,2-Selective Hydrosilylation of Conjugated Dienes. Doctoral dissertation, Harvard University.

\section{Permanent link}

http://nrs.harvard.edu/urn-3:HUL.InstRepos:12274560

\section{Terms of Use}

This article was downloaded from Harvard University's DASH repository, and is made available under the terms and conditions applicable to Other Posted Material, as set forth at http:// nrs.harvard.edu/urn-3:HUL.InstRepos:dash.current.terms-of-use\#LAA

\section{Share Your Story}

The Harvard community has made this article openly available.

Please share how this access benefits you. Submit a story.

Accessibility 
A dissertation presented

by

Sarah Elizabeth Parker

to

The Department of Chemistry and Chemical Biology

\author{
in partial fulfillment of the requirements \\ for the degree of \\ Doctor of Philosophy \\ in the subject of \\ Chemistry
}

Harvard University

Cambridge, Massachusetts

April 2014 
C 2014 Sarah Elizabeth Parker

All rights reserved 


\title{
1,2-Selective Hydrosilylation of Conjugated Dienes
}

\begin{abstract}
Selective 1,2-hydrosilylation of 1,3-dienes is a challenging problem to solve for transition metal catalysis. Butadiene, specifically, would be a useful substrate because 3-butenylsilane products have promise as superior coupling reagents for hybrid organic/inorganic materials synthesis. In this thesis, we describe the first selective 1,2-hydrosilylation of conjugated dienes, including butadiene.

Chapter 1 describes the relevance of organosilicon compounds to modern applications such as the synthesis of advanced materials and fine chemicals. The history and development of transition metalcatalyzed hydrosilylation methods are discussed, with emphasis on the current state of understanding of the mechanisms of late-transition metal-catalyzed hydrosilylation. Known methodologies for the hydrosilylation of dienes and known 1,2-additions to dienes are discussed, highlighting the mechanistic features of these processes that informed the presented work.

In Chapter 2, novel research on the discovery and development of platinum catalysts for the 1,2selective hydrosilylation of dienes is presented. Discovery of a cyclometallated platinum complex of tritert-butylphosphine as a precatalyst enabled the 1,2-selective hydrosilylation of conjugated dienes, including butadiene, isoprene, myrcene, and 2,3-dimethylbutadiene. Catalysis proceeds through a $\mathrm{Pt}(\mathrm{II}) / \mathrm{Pt}(\mathrm{IV})$ catalytic cycle and selectivity for 1,2-addition arises from coordinative saturation at catalyst intermediates that prevents formation of $\pi$-allyl complexes. Data are presented that support the proposed $\mathrm{Pt}(\mathrm{II}) / \mathrm{Pt}(\mathrm{IV})$ mechanism and exclude more conventional $\mathrm{Pt}(0) / \mathrm{Pt}(\mathrm{II})$ mechansims.
\end{abstract}




\section{Table of Contents}

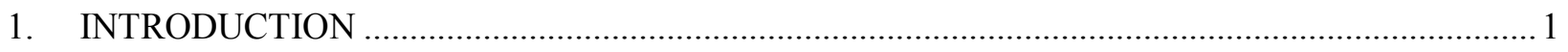

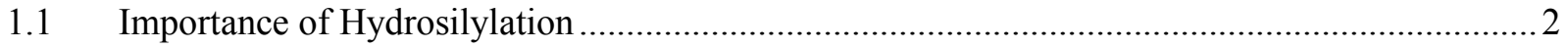

1.1.1. Organosilanes in Sol-Gel Materials Synthesis............................................................... 4

1.1.2. Coupling Agents for Hybrid Materials Synthesis ......................................................... 11

1.1.3. Applications of Organosilanes in Organic Synthesis ..................................................... 17

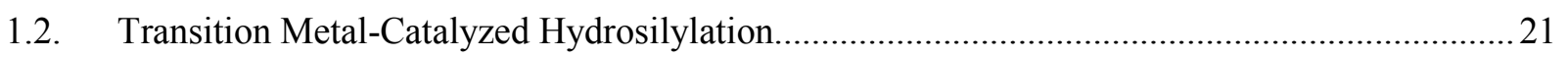

1.2.1. Homogeneous Platinum-Catalyzed Hydrosilylation...................................................... 21

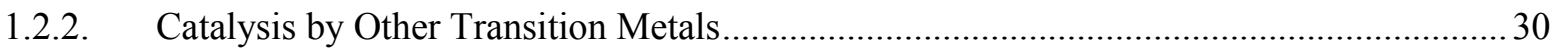

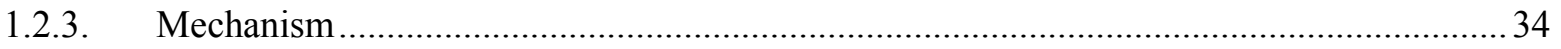

1.3. Transition Metal-Catalyzed Hydrosilylation of Conjugated Dienes......................................... 45

1.3.1. Challenges of Selective Addition to Conjugated Dienes ................................................ 45

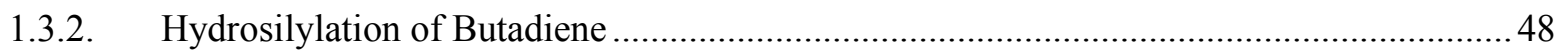

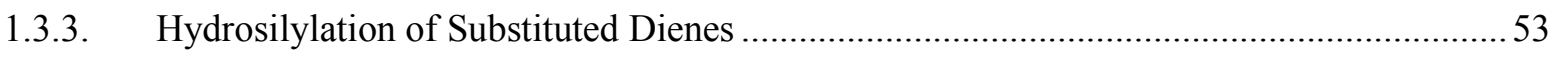

1.3.4. Development of Selective Additions to Conjugated Dienes in the Ritter Research Group 58

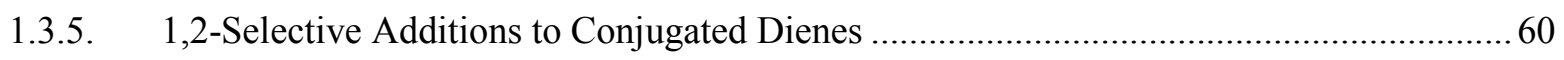

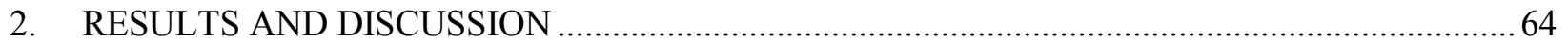

2.1. Strategy for 1,2-Selective Hydrosilylation Catalyzed by Transition Metals ...........................66

2.2. Development of Platinum Catalysts for 1,2-Selective Hydrosilylation of Butadiene................. 68

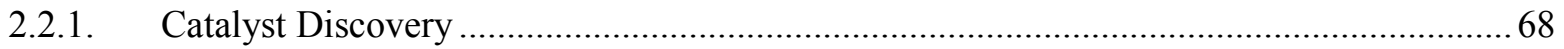

2.2.2. Hydrosilylation of 1,3-Dienes by Cyclometallated Platinum Complexes .........................73

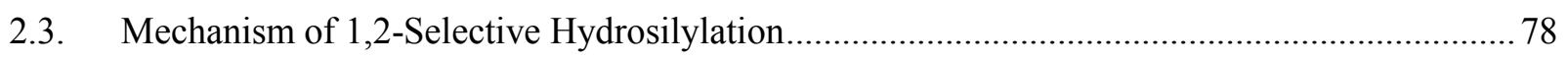

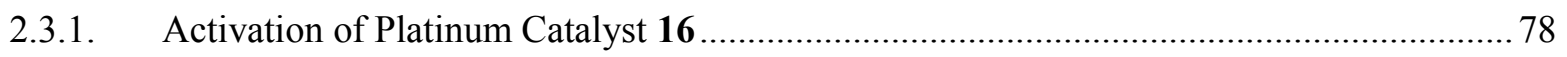

2.3.2. Proposed Mechanism for 1,2-Selective Hydrosilylation................................................. 80

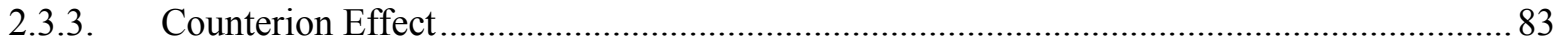

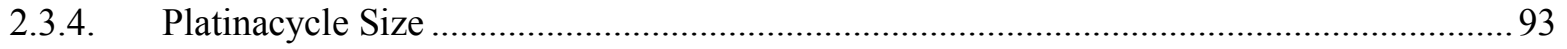

2.3.5. Evidence Against a Chalk-Harrod-Like Pt(0)/Pt(II) Catalytic Cycle ............................... 96

2.3.6. Alternate Mechanism Proposals......................................................................................... 102

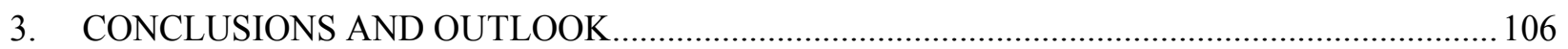

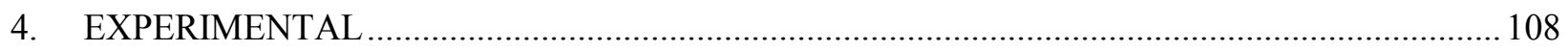

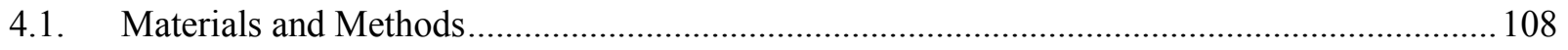




\section{Table of Contents (Continued)}

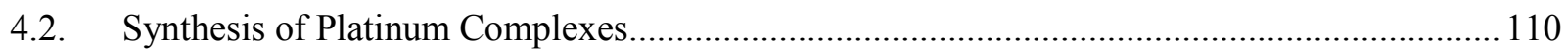

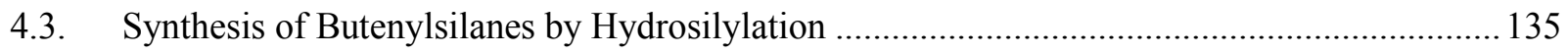

4.4. Synthesis of Reagents, Ligands, and Substrates …............................................................... 143

4.5. Optimization of Hydrosilylation Using Precatalysts 16 and 19 .......................................... 149

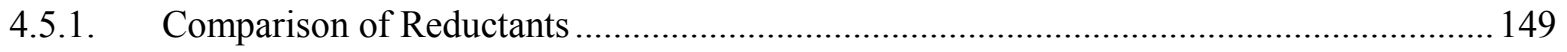

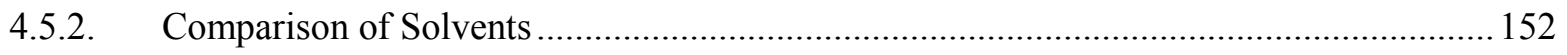

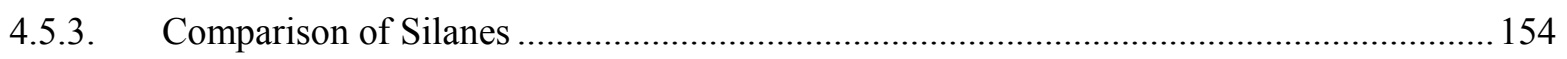

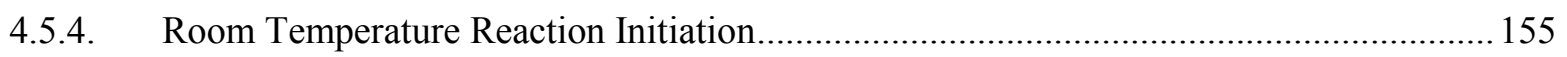

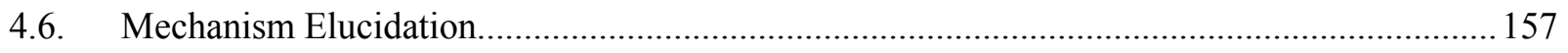

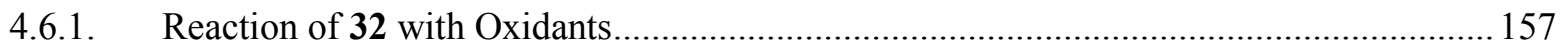

4.6.2. Observation of Catalyst/Ligand Products after Hydrosilylation ...................................... 157

4.6.3. Detection of Methane During Catalyst Activation........................................................ 159

4.6.4. Test of Butenylsilane Reductive Elimination from Precatalyst 36 ............................... 161

4.6.5. Comparison of Platinum Pre-Catalysts ..................................................................... 163

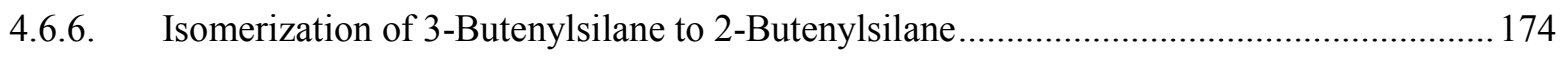

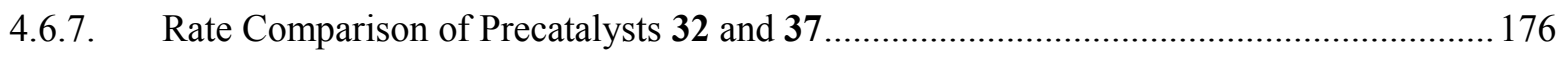

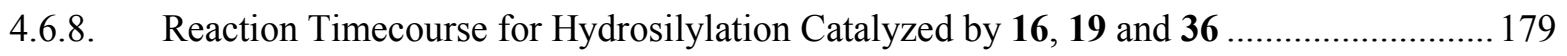




\section{List of Figures and Schemes}

Scheme 1. Synthesis of Hydrophobic Silicate Materials ......................................................................... 9

Scheme 2. Mechanism of Organosilane Condensation on a Silicate Surface …...................................... 13

Scheme 3. Mechanism of Adhesion by Covalent Coupling Reagents .................................................. 13

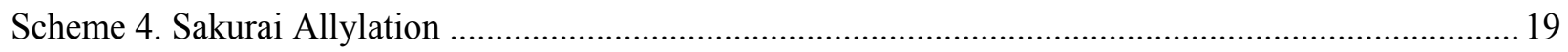

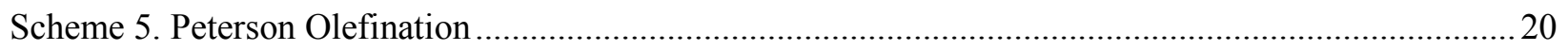

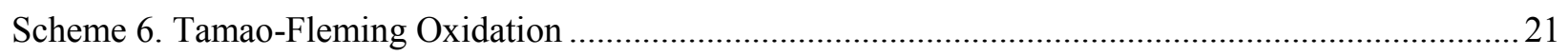

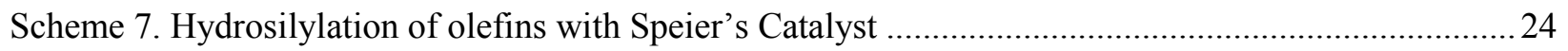

Figure 1. Typical platinum precatalysts for the hydrosilylation of olefins .........................................2

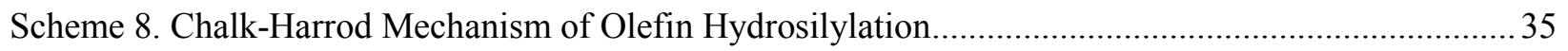

Figure 2. Synthetic analogs of intermediates on the Chalk-Harrod cycle............................................... 38

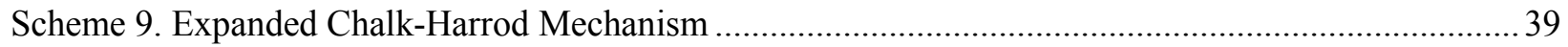

Scheme 10. Common Side-Products of Transition Metal-Catalyzed Hydrosilylation .............................. 40

Scheme 11. Modified Chalk-Harrod Mechanisms for non-Pt catalysts................................................ 42

Scheme 12. Mechanism of Hydrosilylation by Early Transition Metals ...................................................45

Scheme 13. Common Products and Byproducts of 1,3-Diene Hydrosilylation ........................................ 46

Scheme 14. Mechanism of 1,3-Diene Hydrosilylation at Platinum Catalysts ........................................47

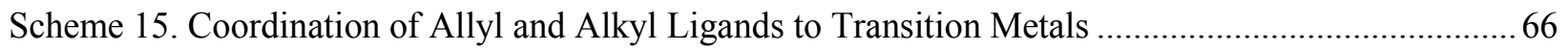

Scheme 16. Migratory Insertion Modes of Conjugated Dienes Bound to Transition Metals ..................... 67

Scheme 17. Strategy for the 1,2-Selective Hydrosilylation of Conjugated Dienes ................................... 68

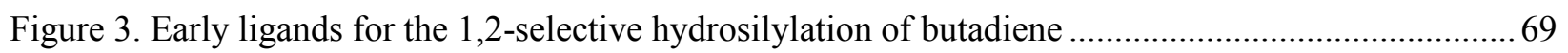

Scheme 18. Mechanism of Cyclometallation at Pt(II) Complexes ......................................................... 71

Scheme 19. Synthesis of Cyclometallated Precatalysts for the Hydrosilylation of Butadiene .................. 73

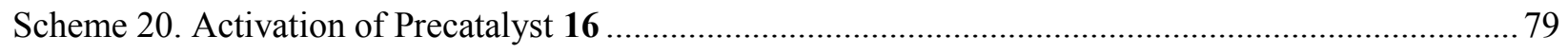

Scheme 21. Proposed Mechanism of Catalyst Activation and Degradation.............................................. 80 


\section{List of Figures and Schemes (Continued)}

Scheme 22. Proposed Mechanism of 1,2-Selective Hydrosilylation ......................................................... 81

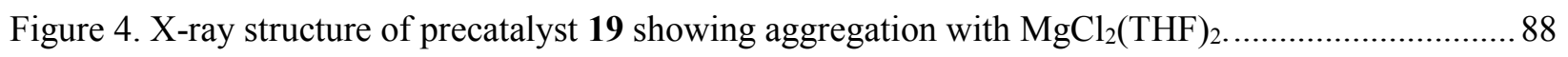

Figure 5. Second $\mathrm{x}$-ray structure of precatalyst 19 showing change in counterion aggregation................. 88

Figure 6. Previously reported anionic platinum(II) complexes. ....................................................... 90

Scheme 23. Synthesis of 5-Membered Platinacycle Precatalysts ......................................................... 94

Scheme 24. Effect of 5-Membered Platinacycle on Catalyst Activation ................................................ 96

Scheme 25. Chalk-Harrod-Like Pt(0)/Pt(II) Mechanism for Hydrosilylation of Butadiene.......................97

Figure 7. Optimized structures for the products of 1,2- vs. 1,4-migratory insertion. ............................. 98

Scheme 26. Attempted Synthesis of $\mathbf{3 6}$ via Dialkenylplatinate Intermediate 43 ..................................99

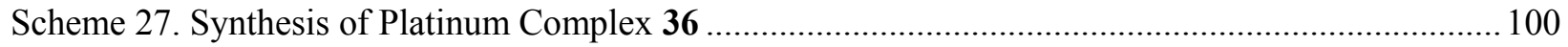

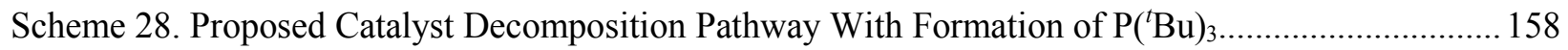

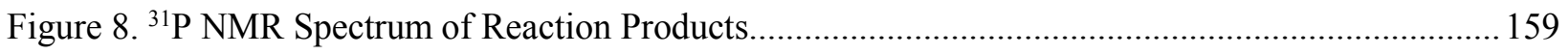

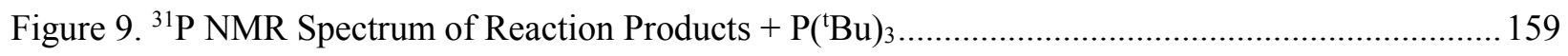

Scheme 29. Proposed Synthesis of Active Catalyst A with Release of Methane ................................... 160

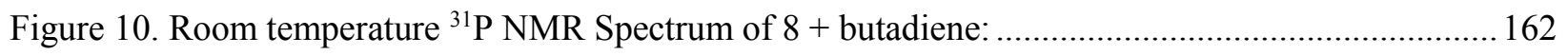

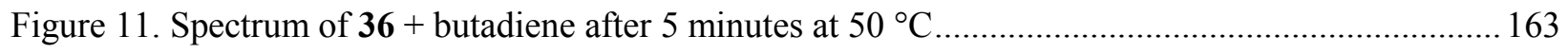

Figure 12. Isomerization of 3-Butenylsilane (18) Using Precatalyst 16 ............................................. 175

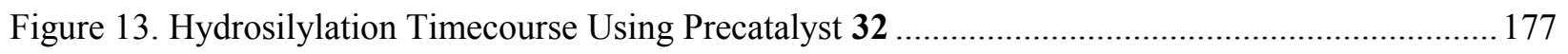

Figure 14. Hydrosilylation Timecourse Using Precatalyst 37 ........................................................ 179

Figure 15. Hydrosilylation Timecourse Using Precatalyst 16 ....................................................... 180

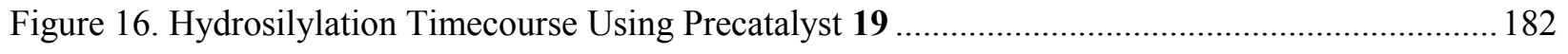

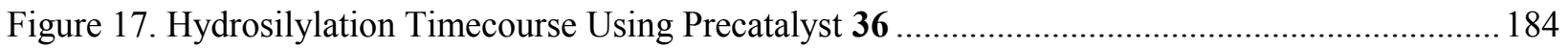




\section{List of Tables}

Table 1. Common Organosilane Coupling Agents ........................................................................... 16

Table 2. Average Monthly Price of Precious Metals .............................................................................. 31

Table 3. Ligands Demonstrating 1,2- vs. 1,4-Selectivity in the Hydrosilylation of Butadiene ..................70

Table 4. 1,2-Selective Hydrosilylation of Substituted Dienes ............................................................. 75

Table 5. Comparison of Precatalysts 16 and 19 for Hydrosilylation of Butadiene..................................76

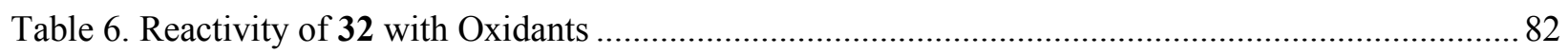

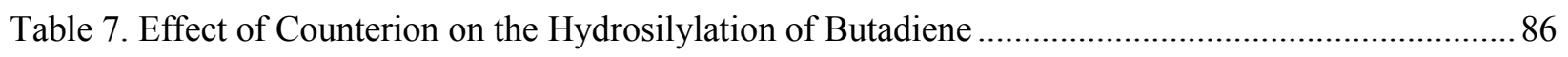

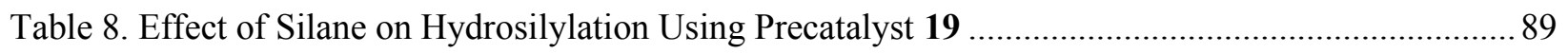

Table 9. Performance of Catalysts Containing Additional Lewis Bases ...............................................92

Table 10. Effect of Solvent on 1,2-Hydrosilylation of Butadiene …....................................................93

Table 11. Comparison of 5-Membered Platinacycle Precatalysts ......................................................... 95

Table 12. Performance of Precatalyst 36 Compared to 16, 19, and 32 .............................................. 101

Table 13. Investigation of $\operatorname{Pt}(0)$ Precatalysts for Hydrosilylation of Butadiene .................................... 103

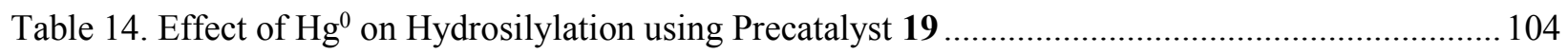

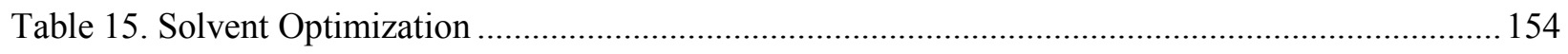

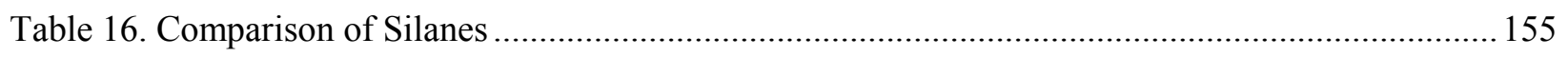

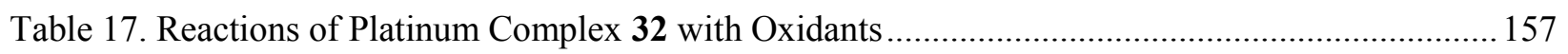

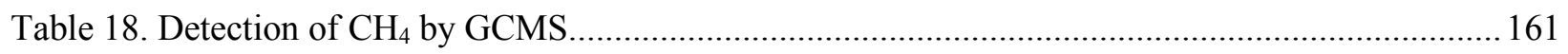




\section{Note}

Portions of this thesis have been taken, with permission, from the following publications:

Parker, S. E.; Börgel, J.; Ritter, T. 1,2-Selective Hydrosilylation of Conjugated Dienes. J. Am. Chem. Soc. 2014, 136, 4857-4860.

Additional work performed while pursuing my Ph.D. that does not appear in this thesis has been published in:

Reynaud, J. B.; Campbell, M. G.; Parker, S. E.; Reese, C. N. A Well-Defined Low-Valent Iron Catalyst for the Practical Synthesis of COD. Submitted. 


\section{Acknowledgements}

The time, science, and effort presented in this thesis would not have been possible without the help, support, friendship, and love of valued coworkers, mentors, friends, and family.

I would like to thank my advisor, Tobias Ritter, for his guidance and support over the past years in the pursuit of science and knowledge. Under his mentorship, I learned how to identify and pursue meaningful goals. I value and appreciate his willingness to engage in honest discussion and his continued attempts to improve himself and his students. I am very grateful to my Graduate Advising Committee members, Eric Jacobsen and Ted Betley, for believing in me and supporting my efforts at every turn. I would especially like to thank Eric Jacobsen for his open-door policy, his advice, and his unwavering belief that I would succeed. I am grateful to Ted Betley for his candid feedback and for his mentorship while teaching the advanced undergraduate inorganic lab course.

I am thankful for the diverse and brilliant coworkers I have had in the Ritter group, who continue to inspire me with their intelligence and drive. During our discussions about science, the world, and life in general, you left a lasting impression that will not swiftly fade. Your ideas and suggestions were indispensable, your companionship and tolerance indispensable. My gratitude extends especially to Nicky Stephenson, Jean Reynaud, Anthony Mazzotti, Kangsang Lee, Eunsung Lee, Filippo Sladojevich, Eric McNeill, Jonas Börgel, Theresa Liang, and Mandeep Virdi for their friendship and support.

Specific thanks are due to Jonas Börgel for his hard work and enthusiasm during his visit to our research group. Jonas was especially helpful in the synthesis of novel platinum complexes of cyclometallated phosphine ligands and in the hydrosilylation of substituted dienes. I would also like to thank Jimmy Sun, who worked with me as an undergraduate researcher in 2012 and 2013. Many thanks as well to Jessica Wu and Mandeep Virdi for their help during my first years in the Ritter group. Although we all had too much to do, they spared time from their own work to teach me laboratory 
techniques and practices. These students also served as valuable role models and I thank them for their independence, drive, and generosity.

My friends and larger community at Harvard were instrumental to my success here. I would like to extend special thanks to Tamara Powers and Libby Hennessey, without whom there would have been many fewer reasons to smile. I cannot express my gratitude for your support and friendship, which has been with me since the first day of new student orientation and will hopefully continue for years to come.

The past few years would have been much less fun without my loving partner Alex Kostadinov. Alex has supported me through some of my most difficult moments and helped me to celebrate some of the best. I am grateful for his love, kindness, and his unwavering commitment to bettering himself and others. I also give the deepest thanks to my parents, Suzanne and Jeff Parker, for their never-ending belief that I would overcome all obstacles and become a successful scientist. You have always been my biggest fans and every success in my life's journey can be attributed to your love and support. I am grateful for everything that you have done, and continue to do, to help me become the best version of myself. 


\section{List of Abbreviations}

*: chiral, single enantiomer

$\delta$ : chemical shift (ppm)

$\mu \mathrm{L}:$ microliter

$\mu$ mol: micromole

Å: Angstrom

A: reactive organic functional group, for example: $-\mathrm{CH}_{2} \mathrm{Cl}$

Ac: acetate

acac: acetylacetonate

Ad: adamantyl

Ar: aryl group or substituent, general

BArF: tetrakis(3,5-

Bn: benzyl

bis(trifluoromethyl)phenyl)borate

Bu: butyl

${ }^{t} \mathrm{Bu}$ : tert-butyl

c: concentration

C: Celsius

calcd: calculated

cat.: catalyst, catalytic amount

COD: 1,5-cyclooctadiene

cont.: continued

$\mathrm{Cp}$ : cyclopentadienyl ligand, bound $\eta^{5}$ unless noted

${ }^{m}$ CPBA: meta-chloroperbenzoic acid

Cy: cyclohexyl

dba: dibenzylideneacetone

DCE: 1,2-dichloroethane

DCM: dichloromethane

$N, N$ '-DMEDA: $N, N$ '-dimethylethylenediamine

DMPF: 1,1'-bis-(dimethylphosphino)ferrocene

dmso: dimethylsulfoxide

DPPE: 1,2-bis(diphenylphosphino)ethane

DPPF: 1,1'-bis(diphenylphosphino)ferrocene

DIPEA: di-iso-propylethylamine

E-: entgegen (olefin isomer)

Eq.: equation

equiv.: equivalent

ESCA: electron spectroscopy for chemical

Et: ethyl analysis

EXAFS: extended x-ray absorption fine structure

g: gram

GC: gas chromatography

GCMS: gas chromatography mass spectrometry
GLYMO: trimethoxy(3-(oxiran-2ylmethoxy)propyl)silane

h: hours

HRMS: high resolution mass spectrometry

Hz: hertz

IR: infrared spectroscopy

$J$ : coupling constant, NMR spectroscopy

L: neutral ligand, general

$L^{*}$ : chiral neutral ligand, single enantiomer

LA: Lewis acid

LUMO: lowest unoccupied molecular orbital

M: metal, general

M: molarity

$[\mathrm{M}+]$ : molecular ion

m: meter

Me: methyl

MEMO: 3-(trimethoxysilyl)propyl methacrylate

Mes: mesityl, 1,3,5-trimethylphenyl

mg: milligram

MHz: megahertz

$\mathrm{mL}$ : milliliter

mmol: millimole

MS: molecular seives

MW: molecular weight

min.: minutes

$\mathrm{n}$ : any integer

n/a: not applicable

NHC: $N$-heterocyclic carbene ligand

NMR: nuclear magnetic resonance spectroscopy

Np: neopentyl

oz.: ounce

$\mathrm{pH}$ : parts hydrogen; measures the acidity of a solution

$\mathrm{Ph}$ : phenyl

$\mathrm{PhH}$ : benzene

PhMe: toluene

Pin: pinacolato-

ppb: parts-per-billion

ppm: parts-per-million

${ }^{i} \mathrm{Pr}$ : iso-propyl

psi: pounds per square inch

py: pyridine

$\mathrm{R}$ : alkyl group or substitutent, general

$\mathrm{R}_{f}$. retention factor (TLC)

$\mathrm{s}$ : seconds

$\mathrm{t}_{R}$ : retention time $(\mathrm{GC}, \mathrm{GCMS})$ 


\section{List of Abbreviations (cont.)}

TBA: tetrabutylammonium

TEM: transmission electron microscopy

Tf: trifluoromethanesulfonyl

THF: tetrahydrofuran

TEOS: tetraethylorthosilicate

TLC: thin-layer chromatography

TMOS: tetramethylorthosilicate

TMS: trimethylsilyl

TOF: turnover frequency

Tol: tolyl

$o$-Tol: ortho-tolyl

TON: turnover number

Tp': hydridotris(3,5-dialkylpyrazolyl)borate

$\mathrm{t}_{R}$ : retention time

UV: ultra-violet light

v/v \%: percent by volume

VTMS: vinyltrimethoxysilane

w/w \%: percent by weight

$\mathrm{X}$ : anionic group or ligand, general

$\mathrm{Y}$ : non-hydrolyzable linking group, such as $\mathrm{CH}_{2} \mathrm{CH}_{2} \mathrm{CH}_{2}-$

$Z$-: zusammen (olefin isomer) 
"The struggle itself toward the heights is enough to fill a man's heart.

One must imagine Sisyphus happy."

$\sim$ Albert Camus 


\section{INTRODUCTION}

Despite the prevalence of transition-metal catalyzed olefin hydrosilylation methods, ${ }^{1}$ no general selective 1,2-hydrosilylation of 1,3-dienes has been reported. Conjugated dienes represent a significant selectivity challenge because various coordination and insertion modes are accessible to a conjugated $\pi$ system at a transition metal catalyst. The rarity of 1,2-selective additions may result from the preference of transition metals to form $\pi$-allyl intermediates upon migratory insertion of dienes into $\mathrm{M}-\mathrm{X}$ bonds, which leads to 1,4 -selectivity. ${ }^{1 \mathrm{~d}, 2}$ Products of 1,2-selective hydrosilylation, specifically 3-butenylsilanes derived from butadiene, would be valuable as superior coupling reagents to link silicate-based materials to olefin polymers in hybrid materials synthesis. ${ }^{1 \mathrm{~d}, 3}$ Previously reported catalysts cannot generate 3-

${ }^{1}$ (a) Speier, J. L.; Webster, J. A.; Barnes, G. H. J. Am. Chem. Soc. 1957, 79, 974. (b) Karstedt, B. D. Ger. Offen. D.E. 2307085 A1, 1973. (c) Harrod, J. F.; Chalk, A. J. In Organic Syntheses via Metal Carbonyls; Wender, I., Pino, P., Eds.; Wiley: 1977; Vol. 2, p 673. (d) Ojima, I.; Kogure, T. Rev. Silicon, Germanium, Tin Lead Compd. 1981, 5, 7. (e) Marciniec, B.; Gulinski, J.; Urbaniak, W.; Kornetka, Z. W. Comprehensive Handbook on Hydrosilylation; Pergamon Press: Oxford, 1992. (f) Brook, M. A. Silicon in Organic Organometallic, and Polymer Chemistry; Wiley-VCH Verlag, 2000. (g) Marciniec, B. Silicon Chem. 2002, 1, 155. (h) Roy, A. K. Adv. Organomet. Chem. 2008, 55, 1. (i) Marciniec, B.; Maciejewski, H.; Pietraszuk, C.; Pawluc, P. In Hydrosilylation: A Comprehensive Review on Recent Advances; Marciniec, B., Ed.; Springer: Dordrecht, 2009; Ch. 1. (j) Troegel, D.; Stohrer, J. Coord. Chem. Rev. 2011, 255,1440 .

2 (a) Shiihara, I.; Hoskyns, W. F.; Post, H. W. J. Org. Chem. 1961, 26, 4000. (b) Mamedaliev, Y. G.; Mamedov, M.; Sadykh-Zade, S. I.; Akhmedov, I. M.; Salimov, M. A. Azerb. Khim. Zh. 1962, 9. (c) Takahashi, S.; Shibano, T.; Hagihara, N. Chem. Commun. 1969, 161. (d) Tsuji, J.; Hara, M.; Ohno, K. Chem. Commun. 1971, 247. (e) Takahashi, S.; Shibano, T.; Kojima, H.; Hagihara, N. Organometal. Chem. Syn. 1971, 1, 193. (f) Belyakova, Z. V.; Pomerantseva, M. G.; Popkov, K. K.; Efremova, L. A.; Golubtsov, S. A. Zh. Obshch. Khim. 1972, 42, 889. (g) Swisher, J. V.; Zullig, C. J. Org. Chem. 1973, 38, 3353. (h) Tsuji, J.; Hara, M.; Ohno, K. Tetrahedron 1974, 30, 2143. (i) Wrighton, M. S.; Schroeder, M. A. J. Am. Chem. Soc. 1974, 96, 6235. (j) Cornish, A. J.; Lappert, M. F. J. Organomet. Chem. 1984, 271, 153. (k) Benkeser, R. A.; Merritt Ii, F. M.; Roche, R. T. J. Organomet. Chem. 1978, 156, 235. (1) Hayashi, T.; Kabeta, K. Tetrahedron Lett. 1985, 26, 3023. (m) Wu, J. Y.; Stanzl, B. N.; Ritter, T. J. Am. Chem. Soc. 2010, 132, 13214.

${ }^{3}$ (a) Arkles, B. CHEMTECH 1977, 7, 766. (b) Plueddemann, E. P. Silane Coupling Agents; 2 ed.; Plenum Press: New York, 1991. (c) Brook, M. A. Silicon in Organic, Oranometallic, and Polymer Chemistry. Wiley-VCH Verlag: 2000; Ch. 10. (d) Ab, R. I.; Padavettan, V. J. Nanomater. 2012, 132424. (e) Cui, S.; Lin, B.; Liu, Y.; Liu, X.; Shen, X.; Han, G.; Zhou, X. Curr. Nanosci. 2012, 8, 797. 
butenyl silanes from an inexpensive and readily-available feedstock, such as butadiene. ${ }^{4}$ This thesis describes the first general 1,2-selective hydrosilylation of conjugated dienes, including butadiene. ${ }^{5}$

This introduction provides a brief history of transition metal-catalyzed hydrosilylation and its relevance as a synthetic method in modern applications. Organosilanes produced by hydrosilylation methods are used as building blocks for advanced silicon-based and hybrid organic/inorganic materials and as tools for fine chemical synthesis. Known methods for the hydrosilylation of 1,3-dienes are reviewed with an emphasis on the hydrosilylation of butadiene. Known 1,2-selective additions to dienes, including hydroboration, diboration, and hydrogenation reactions, are discussed.

\subsection{Importance of Hydrosilylation}

Prior to the discovery of transition metal-catalyzed hydrosilylation, alkyl- and vinylsilanes were obtained by the attack of carbon nucleophiles on silicon halides. The most common method was the addition of a Grignard reagent to a chlorosilane, but other organometallic nucleophiles such as organozinc compounds were also employed. ${ }^{6}$ Although effective for the synthesis of simple organosilanes, nucleophilic displacements were limited in scope by the necessity of using highly reactive carbon-based nucleophiles. Use of Grignard reagents and silyl halides for industrial-scale synthesis is also problematic due to halide-induced reactor corrosion. ${ }^{7}$ The discovery of transition metal-catalyzed hydrosilylation broadened the scope of synthetically accessible alkyl- and vinylsilanes. Currently, hydrosilylation

\footnotetext{
${ }^{4}$ (a) Yamamoto, K.; Nakajima, M.; Shiozawa, M.; Okumura, Y.; Koyama, T. Jpn. Kokai Tokkyo Koho JP 01172391 A, 1989. (b) Brondani, D. J.; Corriu, R. J. P.; El, A. S.; Moreau, J. J. E.; Man, M. W. C. Tetrahedron Lett. 1993, 34, 2111. (c) Deschler, U.; Kleinschmit, P.; Wolff, S.; Tan, E. H. Eur. Pat. Appl. EP 350600 A2, 1990.

${ }^{5}$ Parker, S. E.; Borgel, J.; Ritter, T. J. Am. Chem. Soc. 2014, 136, ASAP.

${ }^{6}$ For reviews of historical methods for Si-C bond formation, see: (a) Corey, J. Y. In The Chemistry of Organic Silicon Compounds; Patai, S., Rappoport, Z., Eds.; John Wiley \& Sons Ltd.: Chichester, 1989; Ch. 1. (b) Birkofer, L.; Stuhl, O. In The Chemistry of Organic Silicon Compounds; Patai, S., Rappoport, Z., Eds.; John Wiley \& Sons Ltd.: Chichester, 1989; Ch. 10.

${ }^{7}$ Lai, G. Y. In High-Temperature Corrosion and Materials Applications; ASM International: 2007; Ch. 6.
} 
processes are used on large scale to synthesize organosilicon compounds for a diverse range of applications, from the synthesis of hybrid materials to organic synthesis. ${ }^{1,8}$

Olefin hydrosilylation is the addition of a silicon-hydride bond across a carbon-carbon double bond, and is among the most important and widely used methods for the synthesis of organosilanes (Eq. $1) \cdot 1,8$

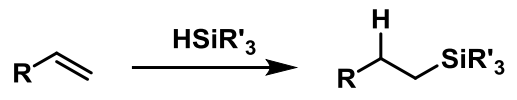

Olefin hydrosilylation was originally discovered by Sommer et al. as a radical-mediated reaction (Eq. 2), ${ }^{9}$ but the transformation was not widely adopted until the discovery of transition metal-catalyzed hydrosilylation by Speier in 1957 (Eq. 3). ${ }^{\text {1a }}$

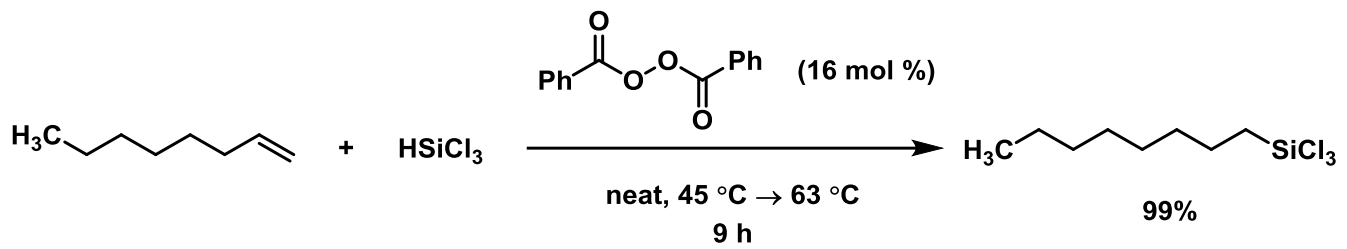

$9 \mathrm{~h}$

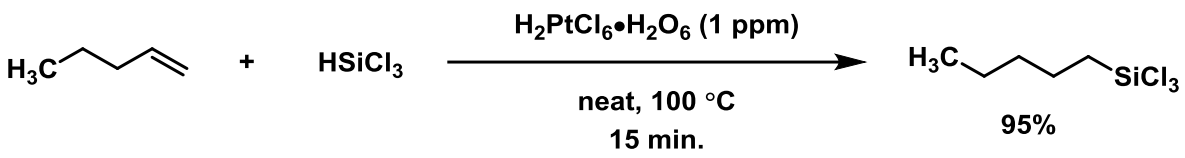

(Eq. 3)

Since then, transition metal catalysts have been developed for the synthesis of a wide variety of organosilanes with remarkable efficiency and selectivity. Carbon-carbon double and triple bonds as well as carbon-heteroatom multiple bonds are now common substrates for hydrosilylation. ${ }^{1}$ Enantioselective

${ }^{8}$ (a) Speier, J. L. Adv. Organomet. Chem. 1979, 17, 407. (b) Ojima, I.; Patai, S., Rappoport, Z., Eds.; Wiley: Chichester, 1989; Ch. 25. (c) Ojima, I.; Li, Z.; Zhu, J. In The Chemistry of Organic Silicon Compounds; Rappoport, Z., Apeloig, Y., Eds.; Wiley: Chichester, 1998; Ch. 29. (d) The Chemistry of Organic Silicon Compounds; Rappoport, Z.; Apeloig, Y., Eds.; John Wiley \& Sons Ltd., 2001; Vol. 3. (e) Marciniec, B. Coord. Chem. Rev. 2005, 249, 2374. (f) Hydrosilylation of Alkenes and Their Derivatives; Marciniec, B.; Maciejewski, H.; Pietraszuk, C.; Pawluc, P., Eds., 2009; Vol. 1.

9 (a) Sommer, L. H.; Pietrusza, E. W.; Whitmore, F. C. J. Am. Chem. Soc. 1947, 69, 188. (b) Speier, J. L.; Zimmerman, R.; Webster, J. J. Am. Chem. Soc. 1956, 78, 2278. 
catalysts with chiral ligands create stereogenic carbon centers through hydrosilylation and provide a facile route to chiral tertiary alcohols. ${ }^{10}$ Hydrosilylation is one of the most mild and functional group-tolerant reactions catalyzed by transition metals and is widely used in the synthesis of fine chemicals and complex organic molecules, as well as on large scale for the production of organosilane building blocks for materials synthesis.

\subsubsection{Organosilanes in Sol-Gel Materials Synthesis}

Silicon-based materials are used in applications ranging from electronics manufacturing to in-vivo drug delivery. ${ }^{11}$ Extensive research in both engineering and chemistry has enabled the precise control of the physical and mechanical properties of silicon-based materials through many synthetic or "bottom up" approaches as well as modification or "top down" methods. Organosilanes are most often used in "bottom up" approaches, specifically in the sol-gel process. Interest in the sol-gel process began with Ebelman ${ }^{12}$ and Graham's ${ }^{13}$ studies of silica gels in the mid-1800's, but was not recognized and developed as a practical method for the synthesis of new and existing inorganic materials until the 1980 's. ${ }^{14}$ In sol-gel synthesis, organosilanes are most commonly incorporated to increase hydrophobicity. Recently, however, functionalized organosilanes called coupling agents that covalently bind to both silicate and organic materials have become widespread. ${ }^{3 b}$ The resulting organofunctionalized silicates are used as reinforced organic polymers (see page 11), for the immobilization of peptides and metal complex catalysts on

${ }^{10}$ For reviews on chiral alcohol synthesis by hydrosilylation, see: (a) Miyake, Y.; Nishibayashi, Y.; Uemura, S. Synlett 2008, 1747. (b) Malacea, R.; Poli, R.; Manoury, E. Coord. Chem. Rev. 2010, 254, 729.

${ }^{11}$ Buriak, J. M. Philos. Trans. R. Soc., A 2006, 364, 217.

${ }^{12}$ (a) Ebelmen, M. Ann. Chimie Phys. 1846, 16, 129. (b) Ebelmen, M. C. R. Acad. Sci. 1847, $25,854$.

${ }^{13}$ Graham, T. J. Chem. Soc., Trans. 1864, 17, 318.

${ }^{14}$ For a review of the principles and methods of sol-gel processing, see: Hench, L. L.; West, J. K. Chem. Rev. 1990, 90, 33. 
inorganic supports, ${ }^{15,16}$ as agents for derivatizing photo electrodes, ${ }^{17}$ as additives for enhancing the mechanical properties of asphalt, ${ }^{18}$ as scratch-resistant surface coatings for polycarbonate lenses, ${ }^{19}$ as hydrophobic glass fibers for reinforcing cement, ${ }^{20}$ as hydrophobic coatings for industrial and automobile glass, ${ }^{3 b}$ as vehicles for pharmaceutical preparations, ${ }^{21}$ and as chromatographic separation solid- and liquid-supports. ${ }^{22}$

The sol-gel process uses readily available siloxanes, such as tetramethylorthosilicate (TMOS), to form highly ordered silicone materials through a hydrolysis/polycondensation mechanism (Eq. 4$){ }^{14}$

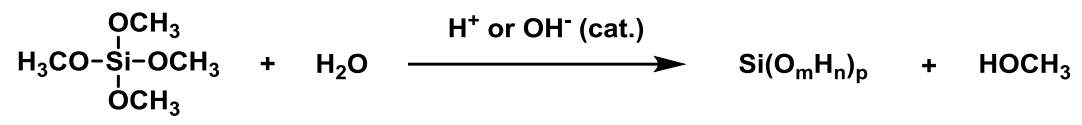

All oxygen atoms in the resulting sol-gel material come from water, and the numbers $m, n$, and $p$ depend on many formulation conditions, including the water/silane ratio, the concentration of acid or base catalyst, the cosolvent, temperature, method and rate of drying, existence of dopants and other additives,

${ }^{15}$ For reviews, see: (a) Schubert, U. New J. Chem. 1994, 18, 1049. (b) Margelefsky, E. L.; Zeidan, R. K.; Davis, M. E. Chem. Soc. Rev. 2008, 37, 1118.

${ }^{16}$ For examples, see: (a) Marciniec, B.; Kornetka, Z. W.; Urbaniak, W. J. Mol. Catal. 1981, 12, 221. (b) Mandoli, A.; Lessi, M.; Pini, D.; Evangelisti, C.; Salvadoria, P. Adv. Synth. Catal. 2008, 350, 375. (c) Puglisi, A.; Annunziata, R.; Benaglia, M.; Cozzi, F.; Gervasini, A.; Bertacche, V.; Sala, M. C. Adv. Synth. Catal. 2009, 351, 219. (d) Puglisi, A.; Benaglia, M.; Annunziata, R.; Chiroli, V.; Porta, R.; Gervasini, A. J. Org. Chem. 2013, 78, 11326.

${ }^{17}$ Bocarsly, A. B.; Walton, E. G.; Bradley, M. G.; Wrighton, M. S. J. Electroanal. Chem. Interfacial Electrochem. 1979, 100, 283.

${ }^{18}$ Crawford, W. C.; Wilson, J. R. U.S. Patent 4173489 A, 1979.

${ }_{19}$ (a) Philipp, G.; Schmidt, H. J. Non-Cryst. Solids 1986, 82, 31. (b) Schmidt, H.; Seiferling, B. Mater. Res. Soc. Symp. Proc. 1986, 73, 739.

${ }^{20}$ Chvalovsky, V. In Carbon-Functional Organosilicon Compounds; Chvalovsky, V., Bellama, J. M., Eds.; Plenum Press: New York, 1984; Ch. 1.

${ }^{21}$ (a) Salonen, J.; Kaukonen, A. M.; Hirvonen, J.; Lehto, V.-P. J. Pharm. Sci. 2008, 97, 632. (b) Santos, H. A.; Hirvonen, J. Nanomedicine 2012, 7, 1281.

${ }^{22}$ Wyndham, K. D.; O'gara, J. E. WO 2008085435 A 1, 2008. 
size and shape of the product, and even the nature of the reaction container or support. ${ }^{23}$ The mechanism of polycondensation is still unknown, but is thought to proceed by a fast hydrolysis to $\mathrm{Si}(\mathrm{OH})_{4}$ followed by slow condensation. ${ }^{24}$ Sol-gel silicates are porous materials with a range of pore sizes centered around the tens of Angstroms and surface areas ranging from a few $\mathrm{m}^{2} \mathrm{~g}^{-1}$ to several hundred $\mathrm{m}^{2} \mathrm{~g}^{-1}$. The surface of the sol-gel material is typically hydrophilic, coated with $\mathrm{Si}-\mathrm{OH}$ groups and adsorbed water molecules. The complex dependence of material properties on the conditions of formation allows the sol-gel process to be extremely versatile. As a result, sol-gel materials can be prepared for many applications. ${ }^{23 g}$

Many silicates contain organic molecules that modify the properties or allow for further modification of the material. ${ }^{25}$ Three general methods have been developed to incorporate organic molecules into silicate materials, including: (a) entrapment of organic molecules in sol-gel matrices, ${ }^{26}$ (b) incorporation of organo- or organofunctionalsilanes as covalently bonded C-Si building blocks, ${ }^{3 \mathrm{~b}}$ and (c)

\footnotetext{
${ }^{23}$ For studies and reviews of factors that influence the physical properties of sol-gel materials, see: (a) Artaki, I.; Bradley, M.; Zerda, T. W.; Jonas, J.; Orcel, G.; Hench, L. L. In Science of Ceramic Chemical Processing; Hench, L. L., Ulrich, D. R., Eds.; Wiley: New York, 1986; p. 73. (b) Hench, L. L.; Orcel, G. J. Non-Cryst. Solids 1986, 82, 1. (c) Orcel, G.; Hench, L. L. In Science of Ceramic Chemical Processing; Hench, L. L., Ulrich, D. R., Eds.; Wiley: New York, 1986; p. 224. (d) Klemperer, W. G.; Mainz, V. V.; Ramamurthi, S. D.; Rosenberg, F. S. In Better Ceramics Through Chemistry III; Brinker, C. J., Clark, D. E., Ulrich, D. R., Eds.; Materials Research Society: Pittsburgh, 1988; p. 15. (e) Klemperer, W. G.; Ramamurthi, S. D. In Better Ceramics Through Chemistry III; Brinker, C. J., Clark, D. E., Ulrich, D. R., Eds.; Materials Research Society: Pittsburgh, 1988; p. 1. (f) Orcel, G.; Hench, L. L.; Artaki, I.; Jonas, J.; Zerda, T. W. J. Non-Cryst. Solids 1988, 105, 223. (g) Brinker, C. J.; Scherer, G. W. Sol-Gel Science : The Physics and Chemistry of Sol-Gel Processing; Academic Press: Boston, 1990.

${ }^{24}$ For NMR and IR studies on the mechanism of sol-gel hydrolysis and condensation, see: (a) Kelts, L. W.; Effinger, N. J.; Melpolder, S. M. J. Non-Cryst. Solids 1986, 83, 353. (b) Zerda, T. W.; Artaki, I.; Jonas, J. J. Non-Cryst. Solids 1986, 81, 365. (c) Smith, D. M.; Glaves, C. L.; Davis, P. J.; Brinker, C. J. In Better Ceramics Through Chemistry III; Brinker, C. J., Clark, D. E., Ulrich, D. R., Eds.; Materials Research Society: Pittsburgh, 1988; p. 657.

${ }^{25}$ For a review of organosilicate sol-gel materials, see: Avnir, D.; Klein, L. C.; Levy, D.; Schubert, U.; Wojcik, A. B. In The Chemistry of Organic Silicon Compounds; Rappoport, Z., Apeloig, Y., Eds.; Wiley: Chichester, 1998; Ch. 40.

${ }^{26}$ For a review, see: Avnir, D. Acc. Chem. Res. 1995, 28, 328.
} 
copolymerization of organic materials with inorganic sol-gel silicates. ${ }^{27}$ All three methods show utility for a variety of applications, but method (b), covalent modification, relies most on the availability of a wide range of organosilane building blocks, many of which are formed by transition metal-catalyzed hydrosilylation reactions.

Synthesis of hybrid organic/inorganic materials by covalent attachment utilizes $\mathrm{R}_{\mathrm{n}} \mathrm{SiX}_{\mathrm{m}}$ building blocks, where $\mathrm{R}$ is a hydrolytically stable organic group and $\mathrm{X}$ is a leaving group such as a halide or alkoxide. The most common precursors used in sol-gel synthesis are trialkoxyorganosilanes, $\mathrm{RSi}(\mathrm{OR})_{3}$, which can be diluted with any desired quantity of $\mathrm{Si}(\mathrm{OR})_{4}$ to control the concentration of organic groups, and resulting properties, of the sol-gel material. The $\mathrm{R}$ group may be any organic group stable to hydrolysis under the condensation conditions, and can be chosen to modify the network structure, introduce organic functionality into the inorganic network, provide a reactive functional group for later modification, or improve the adhesion of hydrophobic materials. ${ }^{20}$

Inclusion of even simple organic functional groups, such as methyl or phenyl groups, into siloxane condensation polymers can impact the properties of the bulk material. ${ }^{28,29}$ When orthosilicates are replaced by organosilanes in the inorganic polymer network, a coordination site is blocked towards hydrolysis and condensation. The incorporation of organosilanes, therefore, lowers the degree of crosslinking in the inorganic network. Because rates of hydrolysis differ for organosilanes and orthosilicates, the composition of the resulting condensation polymer will not be homogeneous.

${ }^{27}$ For reviews describing inorganic/organic copolymers, see: (a) Roy, R. A.; Roy, R. Mater. Res. Bull. 1984, 19, 169. (b) Sanchez, C.; Ribot, F. New J. Chem. 1994, 18, 1007. (c) Mark, J. E. Heterog. Chem. Rev. 1996, 3, 307.

${ }^{28}$ For a review of silsesquioxane properties, see: Baney, R. H.; Itoh, M.; Sakakibara, A.; Suzuki, T. Chem. Rev. 1995, 95, 1409.

${ }^{29}$ For studies and reviews of surface wettability and thermal stability of organosilicon-treated hybrid materials, see: (a) Bascom, W. D.; Romans, J. B. Ind. Eng. Chem., Prod. Res. Develop. 1968, 7, 172. (b) Zisman, W. A. Ind. Eng. Chem., Prod. Res. Develop. 1969, 8, 98. (c) Plueddemann, E. P. J. Adhes. 1970, 2,184 . 
Typically, the orthosilicate will be hydrolyzed faster than the organosilane, ${ }^{30}$ creating a core of crosslinked silicate material that includes little organosilane functionality with a layer of organosilane condensed around the core after the orthosilicate concentration is depleted (Scheme 1) ${ }^{31}$ For this reason, a common effect of organosilane incorporation into silsesquioxane polymers is to increase the surface hydrophobicity of the silsesquioxane. ${ }^{32}$

Reactive organofunctional groups can add complexity to sol-gel materials through chemical modification or post-modification. ${ }^{25,33}$ These building blocks can be described as $(\mathrm{RO})_{3} \mathrm{Si}-\mathrm{Y}-\mathrm{A}$, where $\mathrm{Y}$ is a non-hydrolysable linker such as a propyl hydrocarbon chain and $\mathrm{A}$ is a reactive organic functional group such as an alkyl chloride, alkyne, or olefin. Functional groups on the surface of silicate materials can undergo subsequent reactions to serve many purposes. Among the reactions of functional group A are: crosslinking with existing organofunctional groups, polymerization with added monomer to form a secondary polymeric network, and attachment of new functionality to the surface of the silsesquioxane. The incorporation of organosilanes into silicates and other minerals allows for nearly infinite variation of physical properties, limited only by the availability of new organosilane building blocks

\footnotetext{
${ }^{30}$ For studies of hydrolysis of $\mathrm{Me}_{2} \mathrm{Si}(\mathrm{OEt})_{2}$ and TEOS, see: (a) Babonneau, F.; Thorne, K.; Mackenzie, J. D. Chem. Mater. 1989, 1, 554. (b) Babonneau, F. Polyhedron 1994, 13, 1123.
}

31 For an example in which hydrolysis rate was used to create a multi-layered material, see: (a) Schwertfeger, F.; Glaubitt, W.; Schubert, U. J. Non-Cryst. Solids 1992, 145, 85. (b) Schubert, U.; Schwertfeger, F.; Huesing, N.; Seyfried, E. Mater. Res. Soc. Symp. Proc. 1994, 346, 151. (c) Schwertfeger, F.; Huesing, N.; Schubert, U. J. Sol-Gel Sci. Technol. 1994, 2, 103. (d) Huesing, N.; Schwertfeger, F.; Tappert, W.; Schubert, U. J. Non-Cryst. Solids 1995, 186, 37. (e) Husing, N.; Schubert, U. J. Sol-Gel Sci. Technol. 1997, 8, 807.

32 For examples where fluorinated organosilanes are incorporated into sol-gel materials to increase hydrophobicity, see: (a) Kobayashi, H. Makromol. Chem. 1993, 194, 2569. (b) Kasemann, R.; Schmidt, H. New J. Chem. 1994, 18, 1117. (c) Kron, J.; Amberg-Schwab, S.; Schottner, G. J. Sol-Gel Sci. Technol. 1994, 2, 189.

${ }^{33}$ For reviews of organofunctional silanes in sol-gel materials synthesis, see: (a) Sanchez, C.; Ribot, F. New J. Chem. 1994, 18, 1007. (b) Schubert, U.; Huesing, N.; Lorenz, A. Chem. Mater. 1995, 7, 2010. 

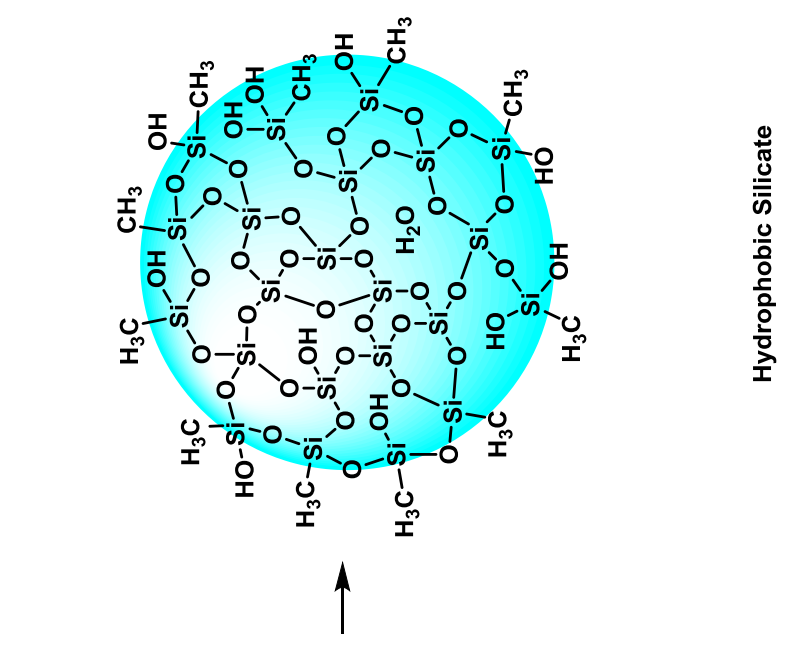


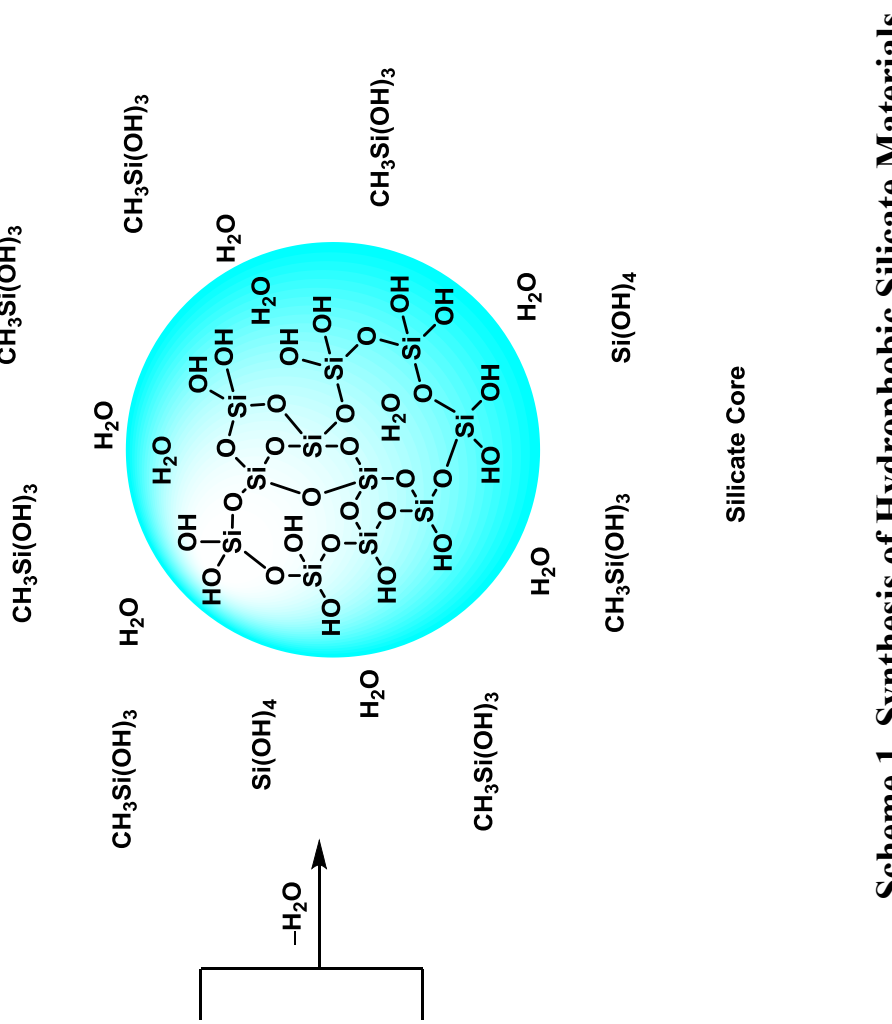

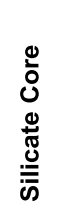

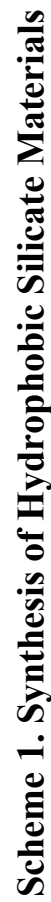

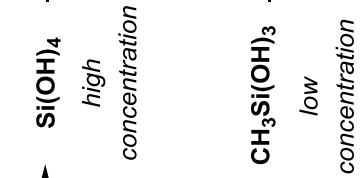

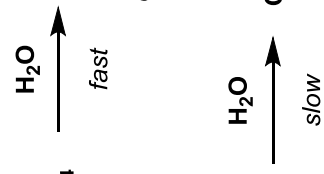

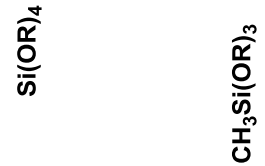


Polymerizable organosilanes are used in combination with orthosilicates for the synthesis of organic/inorganic hybrid materials. ${ }^{25,33}$ Although the components are subjected to sol-gel processing simultaneously, polymerization of the organic group can take place simultaneously or sequentially. A simultaneous polymerization method is used to synthesize a homogeneous, scratch-resistant, transparent coating for optical polycarbonate lenses. ${ }^{19}$ By subjecting trimethoxy(3-(oxiran-2-ylmethoxy)propyl)silane (GLYMO), TMOS, and a metal alkoxide such as $\mathrm{Ti}(\mathrm{OEt})_{4}$ or $\mathrm{Al}(\mathrm{OR})_{3}$ to sol-gel condensation conditions, the construction of silsesquioxane polymer occurs simultaneously with Lewis acid-catalyzed polymerization of the epoxide groups of GLYMO. The result is a homogeneous material comprised of two interpenetrating polymer networks. This approach can also be used with added exogenous organic monomer to increase the conversion of organosilane to organic polymer. ${ }^{34}$ Photochemical polymerization of a curing 3-(trimethoxysilyl)propyl methacrylate (MEMO)/tetrethylorthosilicate (TEOS) gel left approximately $20 \%$ of the methacrylate groups unreacted. Addition of two equivalents of methylmethacrylate during curing, however, resulted in complete polymerization of both the MEMO residues of the gel and the added methylmethacrylate. ${ }^{34 \mathrm{a}}$

An example of co-condensation followed by sequential polymerization is the co-polymerization of multiple organofunctional silanes with TEOS for electronics applications. In this material, the inorganic network is first formed by sol-gel processing a mixture of TEOS, MEMO, vinyltrimethoxysilane (VTMS), and GLYMO. Condensation is followed by UV-initiated polymerization of MEMO in selected areas of the material (photo mask or laser writing). The masked, and therefore nonpolymerized, areas of the coating are then dissolved away before the GLYMO units are thermally polymerized, which sets and strengthens the coating. ${ }^{35}$ Sequential curing methods of this type are useful

\footnotetext{
${ }^{34}$ For examples, see: (a) Delattre, L.; Dupuy, C.; Babonneau, F. J. Sol-Gel Sci. Technol. 1994, 2, 185. (b) Sanchez, C.; Alonso, B.; Chapusot, F.; Ribot, F.; Audebert, P. J. Sol-Gel Sci. Technol. 1994, 2, 161. (c) Sigel, G. A.; Domszy, R. C.; Welch, W. C. Mater. Res. Soc. Symp. Proc. 1994, 346, 135.

${ }^{35}$ Popall, M.; Kappel, J.; Pilz, M.; Schulz, J. Mater. Res. Soc. Symp. Proc. 1992, 264, 353.
} 
for microsystem technology as passivation and encapsulation coatings for electrical components and as protective coatings for thin-film capacitors. ${ }^{36}$

Groups can also be incorporated into sol-gel silicates that remain reactive during the material's end use. For example, materials that function as chemical sensors, ${ }^{37}$ semi-permeable membranes, ${ }^{38}$ chromatographic separation solid phases, ${ }^{22}$ and supports for immobilized transition metal catalysts ${ }^{15,16}$ all require reactive organosilanes at the materials surface to function. For these applications, functional groups such as stimulus-induced chromophores, crown ethers, and amine or phosphine ligands can be used to functionalize the surface of the sol-gel material.

\subsubsection{Coupling Agents for Hybrid Materials Synthesis}

When an organofunctionalsilane is used to increase adhesion of a silicate surface to a dissimilar material, the organofunctionalsilane is called a "coupling agent." ${ }^{3 b}$ Coupling agents are powerful tools used to strengthen the often-weak interface between materials with different physical properties, such as hydrophobic and hydrophilic materials. Organosilane coupling agents were originally developed to increase the adhesion of organic polymers to glass fibers. Glass fibers are incorporated into organic polymers to increase the strength-to-weight ratio of the resulting materials. Although reinforcement with glass fibers produced materials with desirable physical properties, the interface between the organic and inorganic polymers was quickly eroded by water. Sensitivity to water greatly decreased the utility of early

\footnotetext{
${ }^{36}$ Popall, M.; Kappel, J.; Pilz, M.; Schulz, J.; Feyder, G. J. Sol-Gel Sci. Technol. 1994, 2, 157.

${ }^{37}$ For examples, see: (a) Jeng, R. J.; Chen, Y. M.; Jain, A. K.; Kumar, J.; Tripathy, S. K. Chem. Mater. 1992, 4, 972. (b) Nakazumi, H.; Amano, S. Chem. Commun. 1992, 1079. (c) Nosaka, Y.; Tohriiwa, N.; Kobayashi, T.; Fujii, N. Chem. Mater. 1993, 5, 930. (d) Ueda, M.; Kim, H.-B.; Ikeda, T.; Ichimura, K. J. Non-Cryst. Solids 1993, 163, 125. (e) Chambers, R. C.; Haruvy, Y.; Fox, M. A. Chem. Mater. 1994, 6, 1351. (f) Chaput, F.; Boilot, J.-P.; Riehl, D.; Levy, Y. J. Sol-Gel Sci. Technol. 1994, 2, 779. (g) Lebeau, B.; Guermeur, C.; Sanchez, C. Mater. Res. Soc. Symp. Proc. 1994, 346, 315. (h) Tripathy, S. K.; Kumar, J.; Chen, J. I.; Marturunkakul, S.; Jeng, R. J.; Li, L.; Jiang, X. L. Mater. Res. Soc. Symp. Proc. 1994, 346, 531. (i) Yang, Z.; Xu, C.; Wu, B.; Dalton, L. R.; Kalluri, S.; Steier, W. H.; Shi, Y.; Bechtel, J. H. Chem. Mater. 1994, 6, 1899.

${ }^{38}$ Guizard, C.; Lacan, P. New J. Chem. 1994, 18, 1097.
} 
hybrid materials and organosilanes were explored as adhesion promoters to strengthen the interface. Organosilanes were logical adhesion promoters because the organosilane contains properties of both types of materials. Early coupling reagents such as allyltriethoxysilane and vinyltrichlorosilane were very successful. As a result of coupling agent incorporation, hybrid materials could endure hours of incubation in boiling water without eroding the benefits of glass fiber reinforcement. ${ }^{39}$

Most coupling agents are thought to form covalent bonds with both silicates and organic materials, ${ }^{40}$ although examples have been reported in which the formation of covalent bonds is highly unlikely. ${ }^{41}$ For covalently bonded coupling agents, silane hydrolysis to $(\mathrm{HO})_{3} \mathrm{Si}-\mathrm{Y}-\mathrm{A}$ is followed by hydrogen-bonding interaction and finally condensation of the organosilanol group with the silicate surface (Scheme 2). The coupling agent, tethered to the surface, then reacts with organic molecules to form covalent bonds to a preexisting or in situ-generated organic polymer (Scheme 3). Covalent bonding of coupling agents to organic polymers has been demonstrated by FT-IR analysis of styrene-diluted polyester, reinforced by methacrylate- and vinylsilane-coated glass fibers. ${ }^{42}$ Attachment of the organosilanol to the silicate surface was also observed ${ }^{43}$ and these findings were supplemented by model reactions showing quick and efficient bond formation between organosilanol and triethoxysilanol groups. $^{44}$

${ }^{39}$ (a) Erickson, P. W.; Silver, I. U.S. Patent 2776910, 1957. (b) Plueddemann, E. P. J. Paint Technol. 1968, 40,1 .

${ }^{40}$ (a) Wagner, E.; Brunner, H. Angew. Chem. 1960, 72, 744. (b) Anon Eur. Rubber J. 1974, 156, 37. (c) Wagner, M. P. Rubber Chem. Technol. 1976, 49, 703.

${ }^{41}$ For examples, see: (a) Shorr, L. M. J. Am. Chem. Soc. 1954, 76, 1390. (b) Speier, J. L., Jr. U.S. Patent 2723987, 1955. (c) Plueddemann, E. P. Ger. Offen. D.E. 2050467A, 1971. (d) Plueddemann, E. P. Ger. Offen. D.E. 2023968 A, 1971.

${ }^{42}$ Plueddemann, E. P. U.S. Patent 3079361, 1962.

${ }^{43}$ Krahnke, R. H.; Michael, K. W.; Plueddemann, E. P. Ger. Offen. D.E. 2114027 A, 1971.

${ }^{44}$ For model reactions, see: (a) Hertl, W. J. Phys. Chem. 1968, 72, 1248. (b) Dreyfuss, P. Macromolecules 1978, 11, 1031. (c) Dreyfuss, P.; Fetters, L. J.; Gent, A. N. Macromolecules 1978, 11, 1036. 

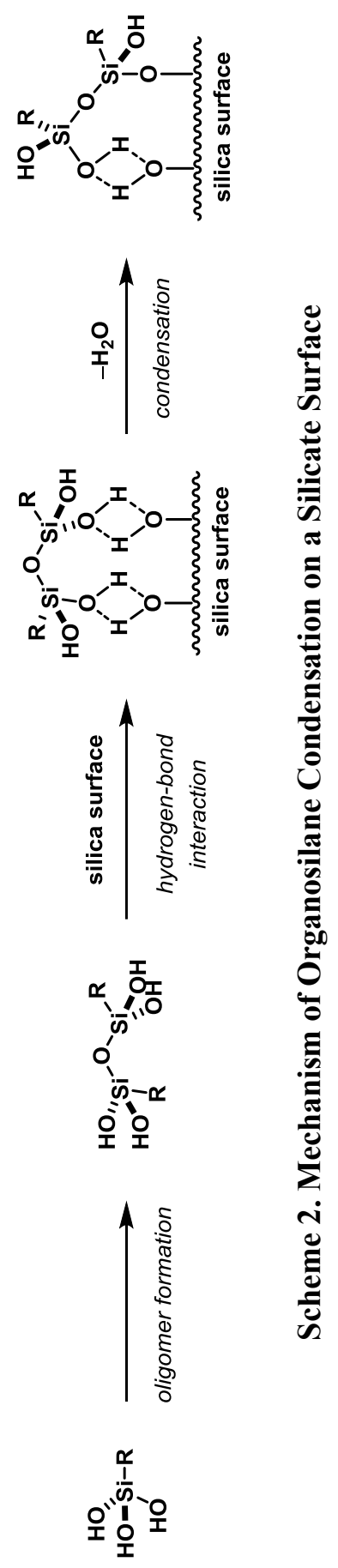
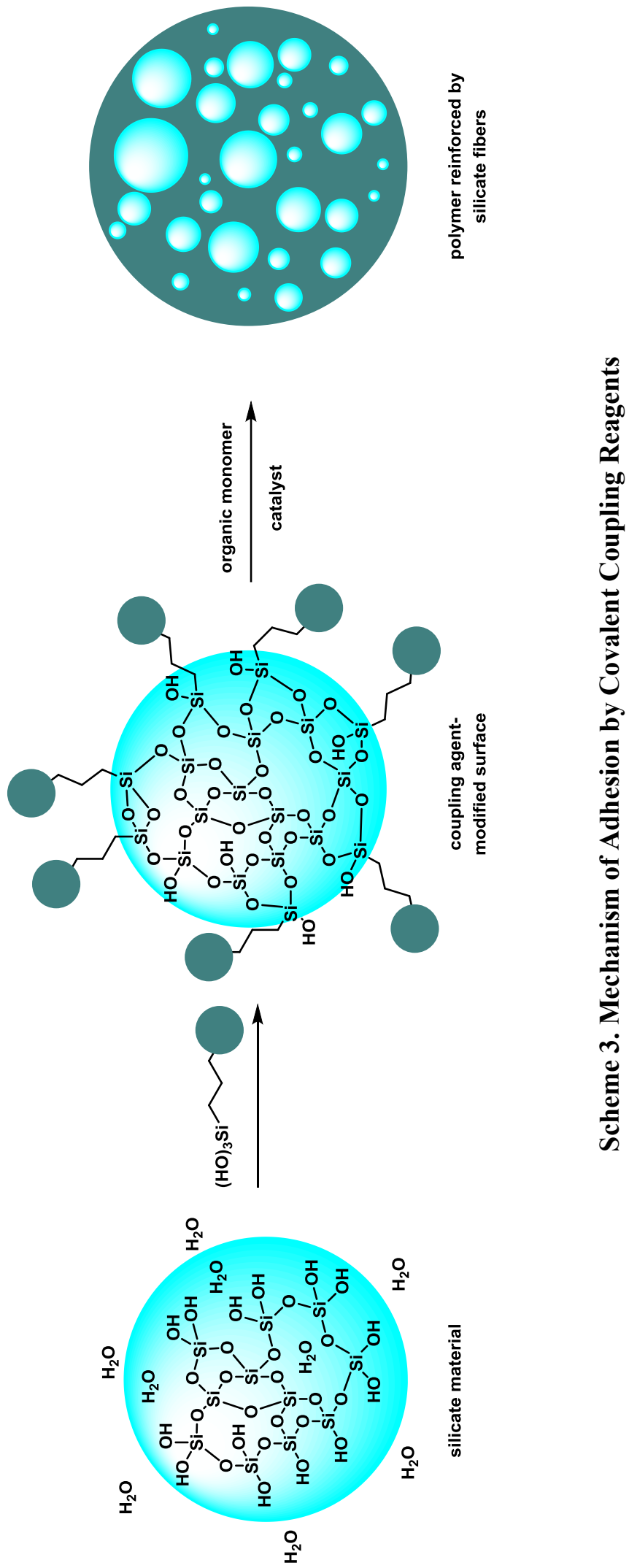
A wide variety of organic functional groups can be incorporated into silane coupling agents for different purposes (Table 1). Functional groups such as alkyl chlorides are useful as coupling reagents for covalent attachment of nucleophilic groups and in polystyrenes ${ }^{45}$ but are more widely used as intermediates in the synthesis of other coupling reagents. ${ }^{3 b}$ The propyl linker in 3-chloropropylsilane coupling agents is necessary to ensure hydrolytic stability of the coupling agent; $\beta$-chlorosilanes are easily cleaved by aqueous base to yield ethylene, $\mathrm{Cl}^{-}$, and a silanol. ${ }^{46}$ Coupling agents bearing unsaturated organofunctional groups such as vinyl, ${ }^{47,48}$ methacryl, ${ }^{48}$ or styrenyl ${ }^{49,50}$ groups are most often used to bond silicates with organic polymers. These coupling agents bear reactive olefins that can participate in either initiation or chain growth of olefin-derived polymers. ${ }^{15,16}$ Nucleophilic $^{51,52,53,54}$ and electrophilic ${ }^{55,56}$ coupling agents

${ }^{45}$ For synthesis of coupling agents from 3-chloropropylsilanes, see: (a) Sommer, L. H.; Strien, R. E. V.; Whitmore, F. C. J. Am. Chem. Soc. 1949, 71, 3056. (b) Speier, J. L., Jr. U.S. Patent 2510148, 1950. (c) Plueddemann, E. P. Fr. Patent F.R. 1509761, 1968. (d) Thomson, J. B. Ger. Offen. D.E. 1965514 A, 1970. (e) Speier, J. L.; Roth, C. A.; Ryan, J. W. J. Org. Chem. 1971, 36, 3120. (f) Isquith, A. J.; Abbott, E. A.; Walters, P. A. Appl. Microbiol. 1972, 24, 859. (g) Berger, A. U.S. Patent 3821218 A, 1974. (h) Speier, J. L. Ger. Offen. D.E. 2543639 A1, 1976. (i) Plueddemann, E. P. U.S. Patent 4093641 A, 1978.

${ }^{46}$ Sommer, L. H.; Whitmore, F. C. J. Am. Chem. Soc. 1946, 68, 485.

${ }^{47}$ Louthan, R. P. U.S. Patent 3890213 A, 1975.

48 (a) Translink 37, Tech. Bull., Freeport Kaolin Co., 1969. (b) Buening, R.; Hanisch, H.; Hass, H. J.; Roedder, K. M. Ger. Offen. D.E. 2730008 A1, 1979.

${ }^{49}$ Plueddemann, E. P. SPI, 27th Ann. Tech. Conf. Reinf. Plast. 1972, 11.

${ }^{50}$ Plueddemann, E. P. SPI, 35th Ann. Tech. Conf. Reinf. Plast. 1980, 20.

${ }^{51}$ (a) Plueddemann, E. P. B.E. 824644 A1, 1975. (b) Le Grand, D. G.; Vitale, G. G. Ger. Offen. D.E. 2643243 A1, 1977. (c) Pepe, E. J.; Marsden, J. G. U.S. Patent 4046794 A, 1977. (d) Plueddemann, E. P. U.S. Patent 4071546 A, 1978.

52 (a) Plueddemann, E. P.; Stark, G. L. Mod. Plast. 1977, 54, 76. (b) Plueddemann, E. P. U.S. Patent 4617057 A, 1986.

${ }^{53}$ (a) Hurd, C. D.; Gershbein, L. L. J. Am. Chem. Soc. 1947, 69, 2328. (b) Patent N.L. 6516641, 1966.

${ }^{54}$ Plueddemann, E. P. J. Adhes. Sci. Technol. 1989, 3, 131.

${ }^{55}$ Plueddemann, E. P.; Fanger, G. J. Am. Chem. Soc. 1959, 81, 2632. 
are often used as synthetic intermediates to form more complex organofunctional silanes, but can also be used in reinforced epoxide polymers.

Many of the coupling agents shown in Table 1 are synthesized efficiently and in a cost-effective manner by transition metal-catalyzed hydrosilylation. Despite extensive research in the field of hydrosilylation, however, substantial challenges remain that directly affect the availability of desirable coupling agents. The synthesis of 3-chloropropylsilanes from allyl chloride, for example, remains challenging because of facile allylic rearrangements at the transition metal catalyst. ${ }^{1 j}$ Hydrosilylation of the allyl group in allylacrylate also yields a useful coupling agent, but allyl acrylate is a challenging hydrosilylation product because of the multiple unsaturated sites available for hydrosilylation. ${ }^{1 \mathrm{j}}$ The hydrosilylation of butadiene to generate 3-butenylsilanes is a third process that, although potentially very useful, is challenging with current transition metal catalysts.

3-Butenylsilanes are potentially valuable as coupling agents but cannot be synthesized in a costeffective manner. Hydrosilylation of butadiene in a 1,2-selective fashion could be a facile route to such coupling agents, but known transition metal catalysts cannot generate the 1,2-hydrosilylation product. Often, transition metal-catalyzed hydrosilylation of 1,3-dienes is unselective, yielding mixtures of isomeric products and mono- and di-addition products (see page 49). When selective for a single product, known transition metal catalysts add $\mathrm{Si}-\mathrm{H}$ bonds to dienes in a 1,4-fashion, generating allylsilane products. Although valuable for a number of applications, ${ }^{57}$ allylsilanes are not effective coupling agents for reinforced olefin-derived polymers because of their low activity as initiation sites for olefin polymerization. Terminal olefins generally show much higher activity as initiation sites, but hydrosilylation to form terminal olefins requires non-conjugated diene starting materials which are expensive relative to butadiene. Currently, vinylsilanes are most often used as coupling reagents for

\footnotetext{
${ }^{56}$ Plueddemann, E. P. U.S. Patent 3057901, 1962.

${ }^{57}$ Luh, T.-Y.; Liu, S.-T. In The Chemistry of Organic Silicon Compounds; Rappoport, Z., Apeloig, Y., Eds.; Wiley: Chichester, 1998; Ch. 30.
} 
olefin-derived polymers despite their low activity towards olefin polymerization, because vinylsilanes can be inexpensively synthesized by hydrosilylation of acetylene. ${ }^{3 \mathrm{~b}}$

Table 1. Common Organosilane Coupling Agents

Coupling Agent

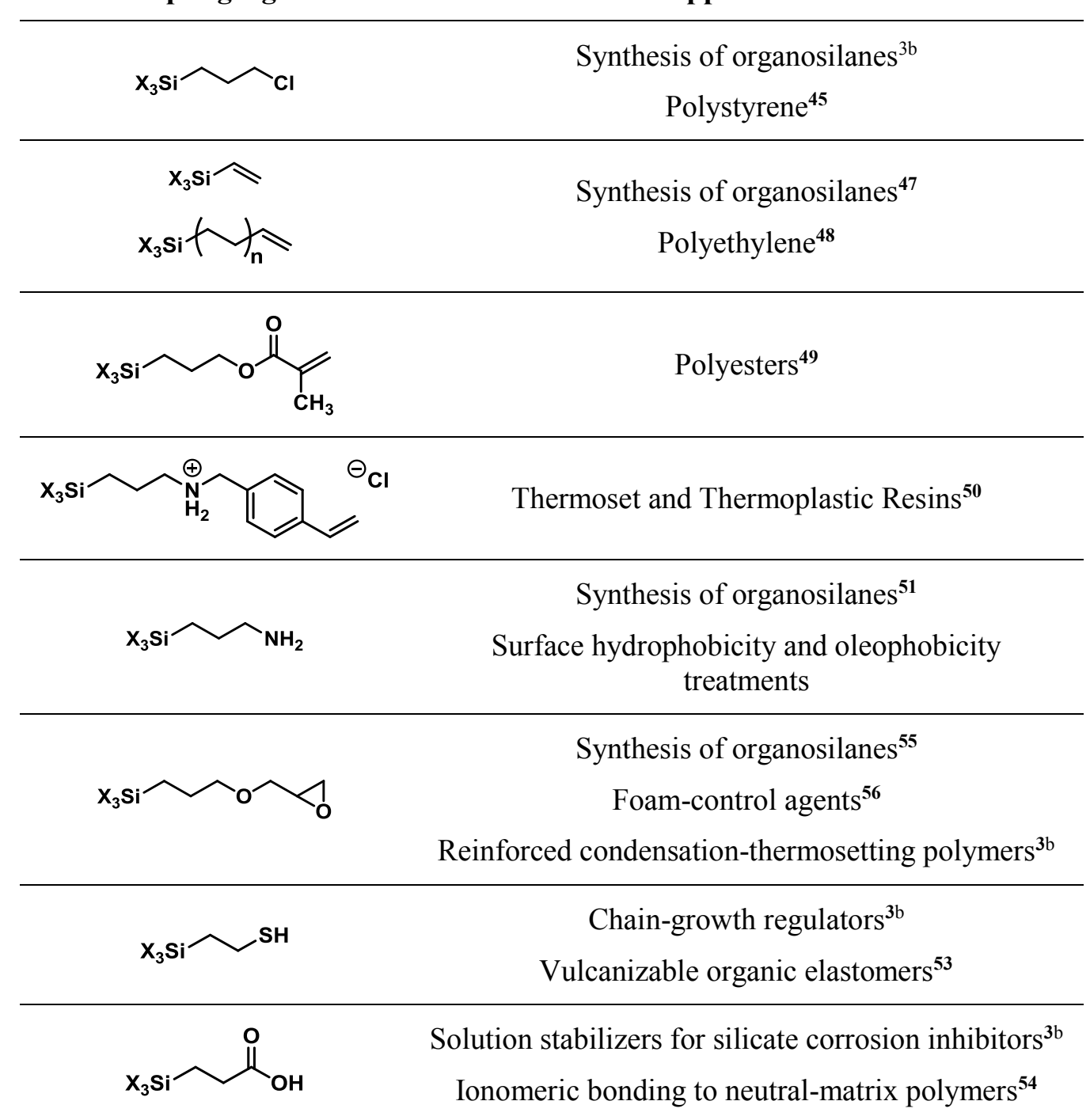




\subsubsection{Applications of Organosilanes in Organic Synthesis}

Although natural products do not contain silicon, methods that utilize organosilanes have become invaluable in organic synthesis ${ }^{58}$ for the introduction of $\mathrm{C}-\mathrm{C}$ and $\mathrm{C}-\mathrm{O}$ bonds, the reduction of ketones to chiral secondary alcohols, protection of polar functional groups, and more. ${ }^{1 \mathrm{f}}$ Silicon(IV) is relatively inert towards many reagents but forms strong bonds to electronegative elements such as oxygen and fluorine. This combination of properties allows $\mathrm{Si}-\mathrm{C}$ and $\mathrm{Si}-\mathrm{O}$ bonds to be robust functional groups that react chemoselectively under appropriate conditions. Silicon also participates in a number of unique rearrangements driven by the strength of silicon-oxygen bonds. Many of the organosilanes utilized in organic transformations are synthesized by transition metal-catalyzed hydrosilylation. The expansion of hydrosilylation processes in recent decades has provided a wealth of new organosilanes and facilitated the growth of organosilicon chemistry in organic synthesis. It is beyond the scope of this introduction to provide a comprehensive review of all organic methods that utilize organosilanes; however, to emphasize the importance of organosilane reagents in organic synthesis, select methods will be described briefly in this section.

Organosilicon reagents are widely utilized for $\mathrm{C}-\mathrm{C}$ bond formation by a variety of transformations including transition metal-catalyzed cross-couplings, ${ }^{59}$ olefinations, ${ }^{60}$ allylations, ${ }^{61}$

\footnotetext{
${ }^{58}$ For reviews on the uses of silicon reagents in organic synthesis, see: (a) Colvin, E. W. Silicon Reagents in Organic Synthesis; Academic Press Limited: San Diego, 1988. (b) Colvin, E. W. In The Chemistry of Organic Silicon Compounds; Rappoport, Z., Apeloig, Y., Eds.; Wiley: Chichester, 1998; Ch. 28.

${ }^{59}$ For seminal work and subsequent reviews of Hiyama coupling, see: (a) Hatanaka, Y.; Hiyama, T. $J$. Org. Chem. 1988, 53, 918. (b) Hiyama, T. J. Organomet. Chem. 2002, 653, 58. (c) Denmark, S. E.; Regens, C. S. Acc. Chem. Res. 2008, 41, 1486.

${ }^{60}$ For seminal work on the Peterson olefination, see: Peterson, D. J. J. Org. Chem. 1968, 33, 780.

${ }^{61}$ For seminal work on the Sakurai allylation, see: (a) Hosomi, A.; Endo, M.; Sakurai, H. Chem. Lett. 1976, 941. (b) Hosomi, A.; Sakurai, H. Tetrahedron Lett. 1976, 1295.
} 
rearrangements, ${ }^{62}$ and cycloadditions. ${ }^{57,63}$ Among the useful C-C bond forming reactions of organosilicon reagents are the Sakurai allylation ${ }^{64}$ and the Peterson olefination reactions. ${ }^{65}$

The Sakurai allylation is a powerful method for the diastereoselective formation of allylic alcohols from ketones and aldehydes. ${ }^{61,64}$ Allyl silanes are readily available by the regioselective $1,4-$ hydrosilylation of 1,3-dienes (see section 1.3). In the Sakurai allylation, Lewis acid activation of a ketone or aldehyde induces addition of the allyl silane to the carbonyl group (Scheme 4). The resulting $\beta$ silylcarbocation is stabilized by hyperconjugation and undergoes elimination by attack of an anion on the silicon center to form the product homoallylic alcohol. The Sakurai allylation has been used in the synthesis of many natural products including furaquinocins, ${ }^{66}(-)$-amphidinolide $\mathrm{P},{ }^{67}$ and $(-)$ laulimalide. $^{68}$

${ }^{62}$ For seminal work and subsequent reviews on the Brook rearrangement, see: (a) Brook, A. G. J. Am. Chem. Soc. 1958, 80, 1886. (b) Brook, A. G.; Warner, C. M.; Mcgriskin, M. E. J. Am. Chem. Soc. 1959, 81, 981. (c) Brook, A. G.; Schwartz, N. V. J. Am. Chem. Soc. 1960, 82, 2435. (d) Schwartz, N. V.; Brook, A. G. J. Am. Chem. Soc. 1960, 82, 2439. (e) Brook, A. G.; Iachia, B. J. Am. Chem. Soc. 1961, 83, 827. (f) Brook, A. G. Acc. Chem. Res. 1974, 7, 77.

${ }^{63}$ For reviews on the use of organosilanes for $\mathrm{C}-\mathrm{C}$ bond formation in organic synthesis, see: (a) Larson, G. L. In The Chemistry of Organic Silicon Compounds; Patai, S., Rappoport, Z., Eds.; John Wiley \& Sons Ltd.: Chichester, 1989; Ch. 11. (b) Larson, G. L. PharmaChem 2010, 9, 26.

${ }^{64}$ For reviews on the Sakurai allylation, see: (a) Schinzer, D. Synthesis 1988, 263. (b) Fleming, I.; Dunogues, J.; Smithers, R. Org. React. 1989, 37, 57. (c) Dai, L.-X.; Lin, Y.-R.; Hou, X.-L.; Zhou, Y.-G. Pure Appl. Chem. 1999, 71, 1033.

${ }^{65}$ For reviews on the Peterson olefination, see: (a) Chan, T.-H. Acc. Chem. Res. 1977, 10, 442. (b) Ager, D. J. Org. React. 1990, 38. (c) Barrett, A. G. M.; Hill, J. M.; Wallace, E. M.; Flygare, J. A. Synlett 1991, 764. (d) Van Staden, L. F.; Gravestock, D.; Ager, D. J. Chem. Soc. Rev. 2002, 31, 195. (e) Ager, D. J. Sci. Synth. 2010, $47 a, 85$.

${ }^{66}$ Trost, B. M.; Thiel, O. R.; Tsui, H.-C. J. Am. Chem. Soc. 2003, 125, 13155.

${ }^{67}$ Williams, D. R.; Myers, B. J.; Mi, L. Org. Lett. 2000, 2, 945.

${ }^{68}$ Wender, P. A.; Hegde, S. G.; Hubbard, R. D.; Zhang, L. J. Am. Chem. Soc. 2002, 124, 4956. 

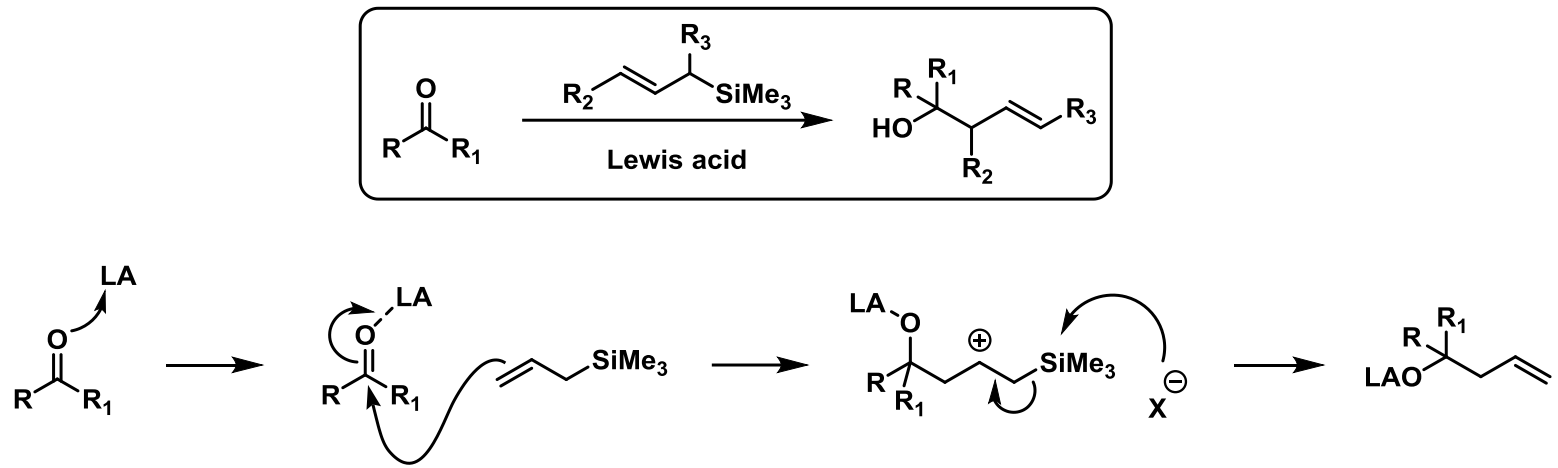

Scheme 4. Sakurai Allylation

The Peterson olefination is a powerful method for the regioselective synthesis of olefins from $\beta$ hydroxysilanes, which are formed from silyl ylides and carbonyl groups ${ }^{60,65}$ For relatively electron-rich substrates, the $\beta$-hydroxysilane intermediate is long-lived and can be isolated. Regioselectivity for longlived $\beta$-hydroxysilane elimination is controlled by the reaction $\mathrm{pH}$, allowing the synthesis of either $E$ - or $Z$-olefins from a single $\beta$-hydroxysilane precursor (Scheme 5). Under basic conditions, a stereospecific concerted syn-elimination is thought to proceed through a 4-membered cyclic intermediate and generates a cis- or Z-olefin. Under acidic conditions, an E2-elimination pathway operates and generates the transor $E$-olefin. Electron-poor $\beta$-hydroxysilanes, however, eliminate quickly and only the $Z$-olefin products can be isolated. The Peterson olefination has been used in the synthesis of several natural products, including the synthesis of $(+)$-maritimol, ${ }^{69}$ the macrocyclic core of roseophilin, ${ }^{70}(+)$-brasilenyne, ${ }^{71}$ and lancifolol. ${ }^{72}$

${ }^{69}$ Toró, A.; Nowak, P.; Deslongchamps, P. J. Am. Chem. Soc. 2000, 122, 4526.

${ }^{70}$ Harrington, P. E.; Tius, M. A. Org. Lett. 1999, 1, 649.

${ }^{71}$ Denmark, S. E.; Yang, S.-M. J. Am. Chem. Soc. 2002, 124, 15196.

${ }^{72}$ Galano, J. M.; Audran, G.; Monti, H. Tetrahedron Lett. 2001, 42, 6125. 


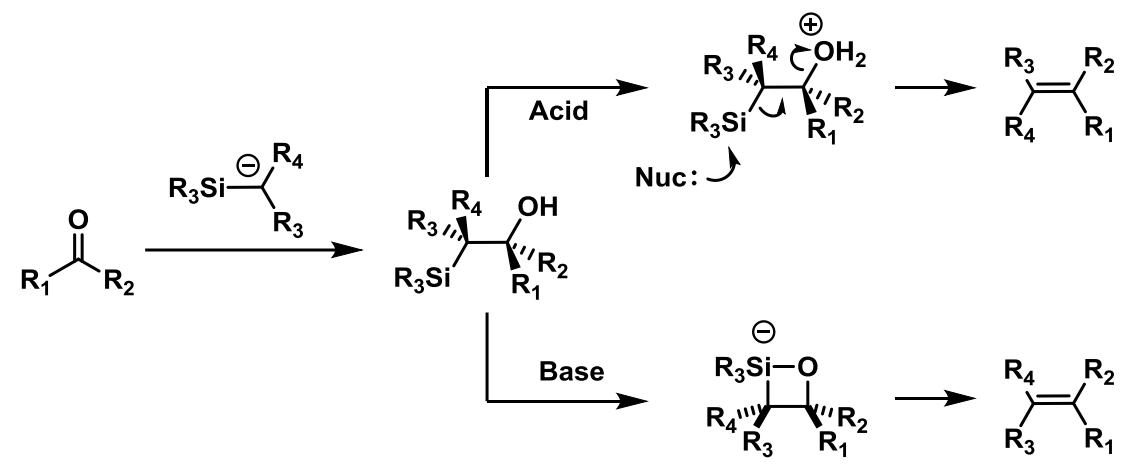

Scheme 5. Peterson Olefination

The Tamao-Fleming oxidation transforms a $\mathrm{C}-\mathrm{Si}$ bond into a $\mathrm{C}-\mathrm{O}$ bond through peroxyacid or peroxide oxidation. ${ }^{73,74}$ The Tamao-Fleming oxidation is useful for the preparation of enantiomerically enriched alcohols because the oxidation proceeds stereoselectively with retention of configuration at carbon. Carbon-silicon bonds can be formed enantioselectively by asymmetric hydrosilylation methods and are relatively stable under a variety of reaction conditions, which allows a wide variety of chiral alcohols to be prepared by this method. The Tamao-Fleming oxidation has been used in the synthesis of many complex molecules, including syntheses of hydroxylated quinolizidines, ${ }^{75}( \pm)$-lepadiformine,${ }^{76}$ the core of garsubellin $\mathrm{A},{ }^{77}$ and a subunit of tautomycin. ${ }^{78}$

${ }^{73}$ For seminal work on the Tamao-Kumada-Fleming Oxidation, see: (a) Tamao, K.; Ishida, N.; Tanaka, T.; Kumada, M. Organometallics 1983, 2, 1694. (b) Tamao, K.; Kumada, M.; Maeda, K. Tetrahedron Lett. 1984, 25, 321. (c) Fleming, I.; Sanderson, P. E. J. Tetrahedron Lett. 1987, $28,4229$.

${ }^{74}$ For reviews of Tamao-Kumada-Fleming Oxidation, see: (a) Fleming, I.; Henning, R.; Parker, D. C.; Plaut, H. E.; Sanderson, P. E. J. J. Chem. Soc., Perkin Trans. 1 1995, 317. (b) Jones, G. R.; Landais, Y. Tetrahedron 1996, 52, 7599.

${ }^{75}$ Vanecko, J. A.; West, F. G. Org. Lett. 2002, 4, 2813.

${ }^{76}$ Sun, P.; Sun, C.; Weinreb, S. M. J. Org. Chem. 2002, 67, 4337.

${ }^{77}$ Usuda, H.; Kanai, M.; Shibasaki, M. Org. Lett. 2002, 4, 859.

${ }^{78}$ Marshall, J. A.; Yanik, M. M. J. Org. Chem. 2001, 66, 1373. 

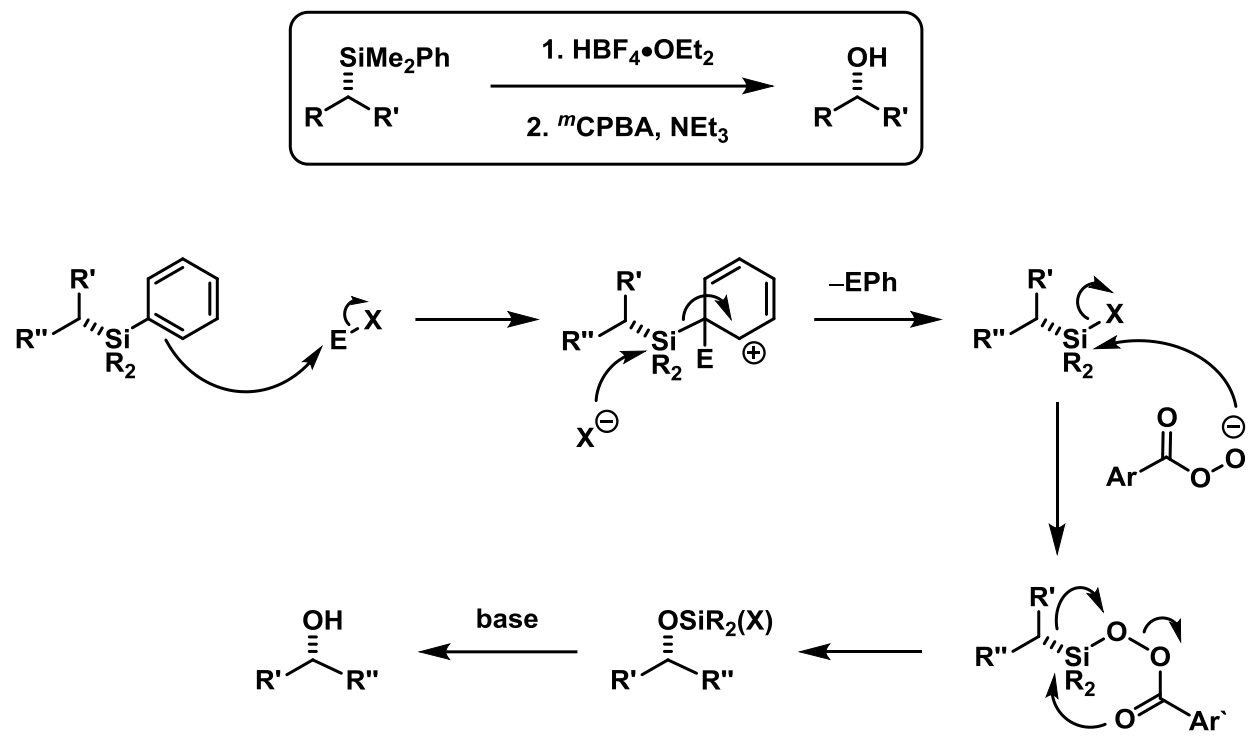

\section{Scheme 6. Tamao-Fleming Oxidation}

Many other organic methods utilize organosilanes as starting materials, reagents, or intermediates to accomplish bond formations and isomerizations. These reactions take advantage of the low polarity of the $\mathrm{C}-\mathrm{Si}$ bond, the ability of the silicon atom to exist as a pentacoordinate silicate, and the thermodynamic driving force of $\mathrm{Si}-\mathrm{O}$ and $\mathrm{Si}-\mathrm{F}$ bond formation. However, the use of organosilicon reagents in fine chemical synthesis cannot begin to compete, in terms of scale or global impact, with the industrial production of silicon-based and hybrid organic/inorganic materials. Both areas of application, however, have greatly benefitted from the development of diverse methods for hydrosilylation.

\subsection{Transition Metal-Catalyzed Hydrosilylation}

\subsubsection{Homogeneous Platinum-Catalyzed Hydrosilylation}

In 1957, Speier, Webster, and Barnes reported the first example of homogeneous transition metalcatalyzed hydrosilylation (Eq. 3). ${ }^{1 \text { a }}$ Previously only free radicals generated by peroxide decomposition, ${ }^{9}$ and heterogeneous metal catalysts such as $\mathrm{Pt} / \mathrm{C},{ }^{79}$ had been shown to catalyze the addition of $\mathrm{Si}-\mathrm{H}$ bonds

${ }^{79}$ (a) Mackenzie, C. A.; Spialter, L.; Schoffman, M. Fr. Patent F.R. 961876, 1947. (b) Wagner, G. H. U.S. Patent 2632013, 1953. (c) Wagner, G. H. U.S. Patent 2637738, 1953. 
to olefins. Speier et al. discovered that hexachloroplatinic acid was more active and selective in the hydrosilylation of olefins than any previously reported catalyst. ${ }^{1 a}$ In subsequent decades, transition metal catalysis has become the method of choice for the synthesis of alkyl- and vinylsilanes, surpassing in scale all methods except the direct process. ${ }^{1 \mathrm{c}-1 \mathrm{i}, 80}$ Although platinum catalysts are the most commonly reported catalysts on large scale, many other metals can catalyze hydrosilylation. Metal catalysts including noble metals such as palladium, ${ }^{81}$ rhodium ${ }^{82}$ ruthenium, ${ }^{83}$ and iridium, ${ }^{84}$ terrestrially abundant metals like cobalt, ${ }^{85}$ nickel, ${ }^{86}$ and iron, ${ }^{2 \mathrm{~m}, 87}$ electrophilic early transition metals, ${ }^{88}$ and lanthanides and actinides ${ }^{89}$ all

${ }^{80}$ The direct process is the copper-catalyzed reaction of alkyl halides with silicon metal, conducted at 300 ${ }^{\circ} \mathrm{C}$, to yield alkyl- or arylsilanes. For seminal work, see: (a) Muller, R. DRP C57411, 1942. (b) Rochow, E. G. J. Am. Chem. Soc. 1945, 67, 963. (c) Hurd, D. T.; Rochow, E. G. J. Am. Chem. Soc. 1945, 67, 1057. (d) Rochow, E. G. U.S. Patent 2380995, 1945. For a general overview of the direct process and its impact on silicon chemistry, see: (e) Brook, M. A.; Wiley-VCH Verlag: 2000; Ch. 12. (f) Seyferth, D. Organometallics 2001, 20, 4978.

${ }^{81}$ For examples, see: (a) Gulinski, J.; James, B. R. J. Mol. Catal. 1992, 72, 167. (b) Widenhoefer, R. A.; Vadehra, A. Tetrahedron Lett. 1999, 40, 8499. (c) Motoda, D.; Shinokubo, H.; Oshima, K. Synlett 2002, 1529.

${ }^{82}$ For examples, see: (a) Millan, A.; Towns, E.; Maitlis, P. M. Chem. Commun. 1981, 673. (b) Onopchenko, A.; Sabourin, E. T.; Beach, D. L. J. Org. Chem. 1984, 49, 3389. (c) Ojima, I.; Clos, N.; Donovan, R. J.; Ingallina, P. Organometallics 1990, 9, 3127. (d) Takeuchi, R.; Tanouchi, N. J. Chem. Soc., Perkin Trans. 1 1994, 2909. (e) Mori, A.; Kato, T. Synlett 2002, 1167. (f) Comte, V.; Le Gendre, P.; Richard, P.; Moïse, C. Organometallics 2005, 24, 1439.

${ }^{83}$ For examples, see: (a) Esteruelas, M. A.; Herrero, J.; Oro, L. A. Organometallics 1993, 12, 2377. (b) Na, Y.; Chang, S. Org. Lett. 2000, 2, 1887. (c) Yardy, N. M.; Lemke, F. R. Main Group Chem. 2000, 3, 143. (d) Trost, B. M.; Ball, Z. T. J. Am. Chem. Soc. 2001, 123, 12726. (e) Martín, M.; Sola, E.; Lahoz, F. J.; Oro, L. A. Organometallics 2002, 21, 4027. (f) Trost, B. M.; Machacek, M. R.; Ball, Z. T. Org. Lett. 2003, 5, 1895.

${ }^{84}$ For examples, see: (a) Esteruelas, M. A.; Olivan, M.; Oro, L. A.; Tolosa, J. I. J. Organomet. Chem. 1995, 487, 143. (b) Klei, S. R.; Tilley, T. D.; Bergman, R. G. Organometallics 2002, 21, 4648. (c) Field, L. D.; Messerle, B. A.; Wren, S. L. Organometallics 2003, 22, 4393. (d) Martín, M.; Sola, E.; Torres, O.; Plou, P.; Oro, L. A. Organometallics 2003, 22, 5406.

${ }^{85}$ For examples, see: (a) Ojima, I.; Clos, N.; Donovan, R. J.; Ingallina, P. Organometallics 1990, 9, 3127. (b) Ojima, I.; Donovan, R. J.; Clos, N. Organometallics 1991, 10, 2606. (c) Hilt, G.; Lueers, S.; Schmidt, F. Synthesis 2004, 634.

${ }^{86}$ For examples, see: (a) Bartik, T.; Nagy, G.; Kvintovics, P.; Happ, B. J. Organomet. Chem. 1993, 453, 29. (b) Tillack, A.; Pulst, S.; Baumann, W.; Baudisch, H.; Kortus, K.; Rosenthal, U. J. Organomet. Chem. 
show activity and unique patterns of selectivity in the hydrosilylation of multiple bonds. Today, transition metal catalysts with a variety of supporting ligands can carry out the selective addition of hydrosilanes to olefins, alkynes, carbonyl groups, imines, nitriles, and more. ${ }^{1 \mathrm{c}-1 \mathrm{i}}$ Despite decades of development, however, significant challenges remain, such as the selective hydrosilylation of allylic electrophiles, conjugated dienes, styrenes, and a variety of other potentially useful substrates..$^{1 \mathrm{j}, 90}$

In 1957, Speier, Webster, and Barnes reported that hexachloroplatinic acid (Speier's catalyst, 1) catalyzes the addition of hydrosilanes to carbon-carbon double bonds. ${ }^{\text {la }}$ Speier's catalyst carries out the anti-Markovnikov addition of hydrosilanes to a variety of simple olefins (Scheme 7).

1997, 532, 117. (c) Maciejewski, H.; Marciniec, B.; Kownacki, I. J. Organomet. Chem. 2000, 597, 175. (d) Fontaine, F.-G.; Nguyen, R.-V.; Zargarian, D. Can. J. Chem. 2003, 81, 1299. (e) Groux, L. F.; Zargarian, D. Organometallics 2003, 22, 4759. (f) Chen, Y.; Sui-Seng, C.; Boucher, S.; Zargarian, D. Organometallics 2004, 24, 149.

${ }^{87}$ For examples, see: (a) Bart, S. C.; Lobkovsky, E.; Chirik, P. J. J. Am. Chem. Soc. 2004, 126, 13794. (b) Tondreau, A. M.; Atienza, C. C. H.; Weller, K. J.; Nye, S. A.; Lewis, K. M.; Delis, J. G. P.; Chirik, P. J. Science 2012, 335, 567.

${ }^{88}$ For examples, see: (a) Corey, J. Y.; Zhu, X. H. Organometallics 1992, 11, 672. (b) Kesti, M. R.; Waymouth, R. M. Organometallics 1992, 11, 1095. (c) Hao, L.; Harrod, J. F.; Lebuis, A.-M.; Mu, Y.; Shu, R.; Samuel, E.; Woo, H.-G. Angew. Chem., Int. Ed. 1998, 37, 3126. (d) Jia, L.; Zhao, J.; Ding, E.; Brennessel, W. W. J. Chem. Soc., Dalton Trans. 2002, 2608. (e) Takahashi, T.; Bao, F.; Gao, G.; Ogasawara, M. Org. Lett. 2003, 5, 3479. (f) Ura, Y.; Gao, G.; Bao, F.; Ogasawara, M.; Takahashi, T. Organometallics 2004, 23, 4804.

${ }^{89}$ For examples, see: (a) Beletskaya, I. P.; Voskoboynikov, A. Z.; Parshina, I. N.; Magomedov, G. K.-I. Bull. Acad. Sci. USSR Div. Chem. Sci. 1990, 39, 613. (b) Sakakura, T.; Lautenschlager, H. J.; Tanaka, M. Chem. Commun. 1991, 40. (c) Molander, G. A.; Romero, J. a. C. Chem. Rev. 2002, 102, 2161.

${ }^{90}$ (a) Smith, A. G.; Ryan, J. W.; Speier, J. L. J. Org. Chem. 1962, 27, 2183. (b) Ryan, J. W.; Speier, J. L. J. Am. Chem. Soc. 1964, 86, 895. 

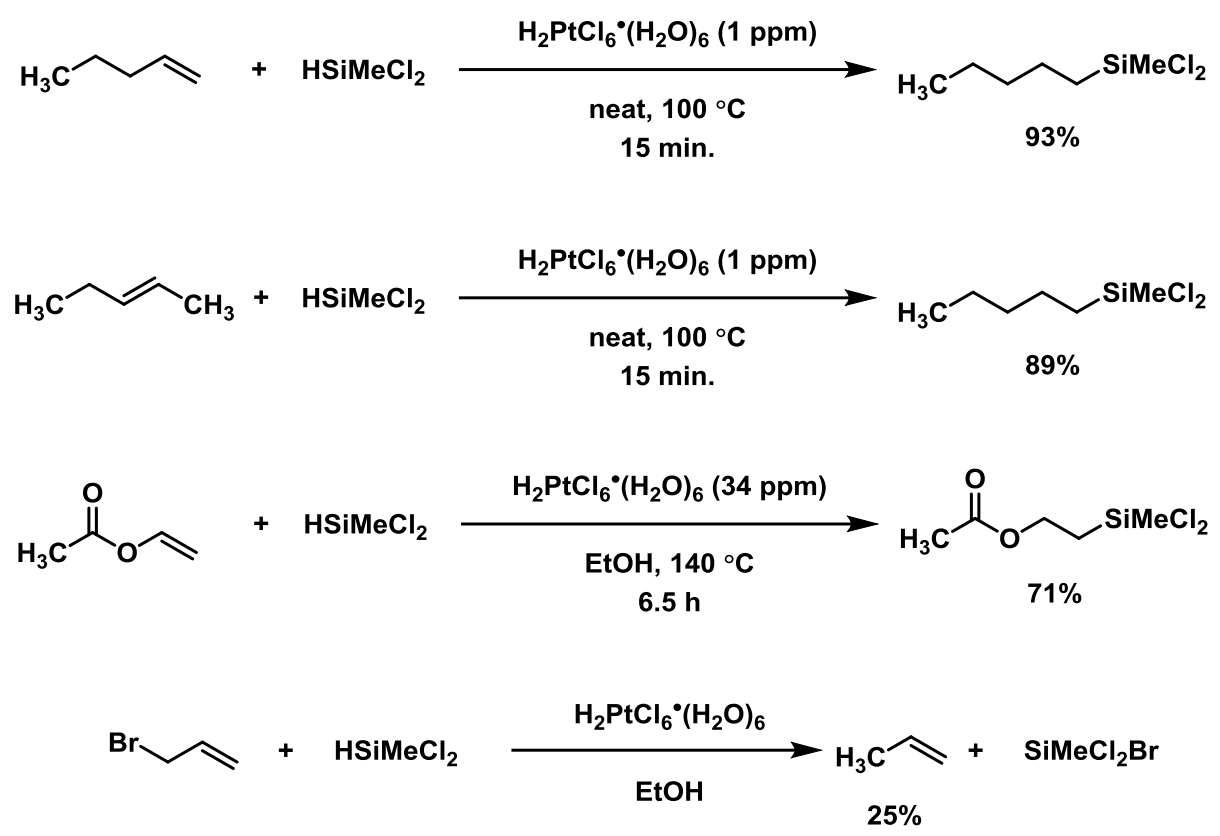

Scheme 7. Hydrosilylation of Olefins With Speier's Catalyst ${ }^{1 \mathrm{a}}$

Although many olefins were competent substrates for hydrosilylation reactions, side reactions were observed in some cases. ${ }^{91}$ Internal olefins, such as 2-pentene, were isomerized to the terminal olefin prior to hydrosilylation, yielding 1-silylpentane in high yields. ${ }^{\text {a,91a,92 }}$ Allylic electrophiles, such as allyl bromide, underwent an exchange reaction with the hydrosilane reagent to yield propene and a silyl halide. ${ }^{91}$ Some of these challenges, such as the hydrosilylation of internal olefins, have been surmounted by platinum catalysts with added ligands or by catalysts of other metals, such as iridium and rhodium. ${ }^{93}$ Other side-reactions have been more difficult to suppress. For example, the reduction of allyl chloride to propene is still problematic in industrial hydrosilylation reactions. The best catalysts for hydrosilylation of allyl chloride can generate $95 \%$ yield of hydrosilylation products with purities of $99 \%$, but the catalysts

${ }^{91}$ (a) Saam, J. C.; Speier, J. L. J. Am. Chem. Soc. 1958, 80, 4104. (b) Saam, J.; Speier, J. J. Am. Chem. Soc. 1961, 83, 1351.

92 Bank, H. M.; Saam, J. C.; Speier, J. L. J. Org. Chem. 1964, 29, 792.

93 Marciniec, B.; Maciejewski, H.; Pietraszuk, C.; Pawluc, P. In Hydrosilylation: A Comprehensive Review on Recent Advances; Marciniec, B., Ed.; Springer: Dordrecht, 2009; Ch. 1. 
are not stable for long periods under the reaction conditions and rapidly lose activity as the reaction progresses. ${ }^{1 \mathrm{j}, 94}$

Catalyst loadings for hydrosilylation reactions using Speier's catalyst can drop as low as $10^{-5}-10^{-8}$ moles catalyst per mole of substrate. Despite the high cost of platinum, Speier's catalyst is used on large scale as a disposable hydrosilylation catalyst because it exhibits extremely high TON (turnover number) and TOF (turnover frequency). ${ }^{1 \mathrm{c}}$ However, Speier's catalyst can be dangerous when used on large scale. In situ reduction of hexachloroplatinic acid to the active $\operatorname{Pt}(0)$ catalyst is unpredictable, which causes reactions catalyzed by Speier's catalyst to exhibit a long induction period followed by a sudden and potentially dangerous exotherm. ${ }^{1 \mathrm{c}, 95}$ Because heat dissipation is inefficient in large reactors, a sudden exotherm can lead to an explosion or to other undesirable outcomes such as the thermal degradation of the hydrosilylation catalyst. ${ }^{1 \mathrm{c}, 96}$

Various cocatalysts and ligands have been added to Speier's catalyst to modify its reactivity and selectivity in the hydrosilylation of olefin substrates. ${ }^{\text {le }}$ Because of the long induction periods during the activation of Speier's catalyst, cocatalysts such as orthotitanates ${ }^{97}$ or metal chloride salts ${ }^{98}$ are sometimes added to facilitate reduction of the precatalyst to $\operatorname{Pt}(0)$. Silanes and unsaturated organic molecules can

${ }^{94}$ Pannetier, G.; Fougeroux, P.; Bonnaire, R. J. Organometal. Chem. 1972, 38, 421.

${ }^{95}$ For a risk assessment of a hydrosilylation reaction employing Speier's catalyst, see: Katoh, K.; Ito, S.; Wada, Y.; Higashi, E.; Suzuki, Y.; Kubota, K.; Nakano, K.; Wada, Y. J. Chem. Thermodyn. 2011, 43, 1229.

${ }^{96}$ For reviews of thermal runaway and related risks in industrial reactors, see: (a) Stoessel, F.; Fierz, H.; Lerena, P.; Kille, G. Org. Process Res. Dev. 1997, 1, 428. (b) Wedlich, R. C. Chem. Eng. Prog. 2001, 97, 60. (c) Wang, Q.; Rogers, W. J.; Mannan, M. S. J. Therm. Anal. Calorim. 2009, 98, 225.

${ }^{97}$ Podol'skii, A. V.; Suvorov, A. L.; Cherezova, T. G.; Fridman, L. I. Zh. Obshch. Khim. 1977, 47, 1532.

${ }^{98}$ For representative examples, see: (a) Warrick, E. L.; Pierce, O. R.; Polmanteer, K. E.; Saam, J. C. Rubber Chem. Technol. 1979, 52, 437. (b) Reichel, S.; Gottfried, R. Ger. Offen. D.E. 2601913 A1, 1976. (c) Voronkov, M. G.; Sushchinskaya, S. P.; Pukhnarevich, V. B. Zh. Obshch. Khim. 1979, 49, 1171. (d) Voronkov, M. G.; Sushchinskaya, S. P.; Pukhnarevich, V. B. Zh. Obshch. Khim. 1981, 51, 242. (e) Voronkov, M. G.; Sushchinskaya, S. P. Zh. Obshch. Khim. 1986, 56, 627. 
also facilitate reduction of the precatalyst. ${ }^{99}$ L-type ligands such as phosphines or amines are added to stabilize the active species toward reductive decomposition or to modify the regioselectivity of hydrosilylation. ${ }^{100}$ For other applications, inhibitors can be added to modify the rate of curing or ageing of hydrosilylation polymers. ${ }^{101}$

Since early mechanistic investigations implicated a $\mathrm{Pt}(0 / \mathrm{II})$ or $\mathrm{Pt}(\mathrm{II} / \mathrm{IV})$ catalytic cycle for hydrosilylation using Speier's catalyst (see section 1.2.3, page 35), many new $\mathrm{Pt}(\mathrm{II})$ and $\mathrm{Pt}(0)$ precatalysts have been developed (Figure 1). Platinum(II) catalysts generally take the form $\mathrm{LPtCl}_{2}$, where $\mathrm{L}$ is an unsaturated $\pi$-accepting ligand such as an olefin, ${ }^{102,103}$ an L-type $\sigma$-donating ligand such as a phosphine or

${ }^{99}$ For representative examples, see: (a) N.L. Patent 6606771, 1966. (b) Kreis, G.; Wegehaupt, K. H. Ger. Offen. D.E. 2846621 A1, 1980. (c) Cavezzan, J. Fr. Patent F.R. 2575086 A1, 1986. (d) Licchelli, M.; Greco, A. Tetrahedron Lett. 1987, 28, 3719. (e) Saruyama, T. Eur. Pat. Appl. E.P. 249944 A2, 1987.

${ }^{100}$ For representative examples, see: (a) Plueddemann, E. P. Fr. Patent F.R. 1509761, 1968. (b) Capka, M.; Svoboda, P.; Hetflejs, J. Collect. Czech. Chem. Commun. 1973, 38, 3830. (c) Brovko, V. S.; Skvortsov, N. K.; Reikhsfel'd, V. O. Zh. Obshch. Khim. 1981, 51, 487. (d) Vaisarova, V.; Hetflejs, J. Synth. React. Inorg. Met.-Org. Chem. 1983, 13, 977. (e) Iovel, I. G.; Gol'dberg, Y. S.; Shimanskaya, M. V.; Lukevics, E. Chem. Commun. 1987, 31. (f) Li, C.; Yuan, G.; Yan, S.; Xie, Z. Yingyong Huaxue 1988, $5,96$.

${ }^{101}$ For a representative example, see: Warrick, E. L.; Pierce, O. R.; Polmanteer, K. E.; Saam, J. C. Rubber Chem. Technol. 1979, 52, 437.

${ }^{102}$ For a review of Pt-alkynyl and Pt-alkyne complexes, see: Belluco, U.; Bertani, R.; Michelin, R. A.; Mozzon, M. J. Organomet. Chem. 2000, 600, 37.

${ }^{103}$ For representative examples, see: (a) Speier, J. L., Jr.; Hook, D. E. U.S. Patent 2823218, 1958. (b) Ashby, B. A. Fr. Patent F.R. 1361705, 1964. (c) Ashby, B. A. Fr. Patent F.R. 1361706, 1964. (d) Koerner, G. Ger. Offen. D.E. 1165028, 1964. (e) Zisman, W. A. Ind. Eng. Chem. 1965, 57, 26. (f) Fr. Patent F.R. 1437798, 1966. (g) N.L. Patent 6606771, 1966. (h) N.L. Patent 6510184, 1966. (i) Koerner, G. Ger. Offen. D.E. 1210844, 1966. (j) Andrianov, K. A.; Petrashko, A. I.; Asnovich, L. Z.; Gashnikova, N. P. Izv. Akad. Nauk SSSR, Ser. Khim. 1967, 1267. (k) Karstedt, B. D. Fr. Patent F.R. 1548775, 1968. (1) Fr. Patent F.R. 1558735, 1969. (m) Fort, G. Ger. Offen. D.E. 1964609, 1970. (n) Joy, J. R. Ger. Offen. D.E. 2152286 A, 1972. (o) Atherton, J. H. Ger. Offen. D.E. 2311879 A1, 1973. (p) Baney, R. H. U.S. Patent 3723497 A, 1973. (q) Baschant, V.; Tschapka, M.; Tscherny, M.; Hetflejsch, J.; Kraus, M.; Svoboda, P. Ger. Offen. D.E. 2245187 A1, 1973. (r) Karstedt, B. D. Ger. Offen. D.E. 2307085 A1, 1973. (s) Plumb, J. B. Ger. Offen. D.E. 2317985 A1, 1973. (t) Hittmair, P.; Hechtl, W.; Eckhart, L.; Wohlfarth, E. Ger. Offen. D.E. 2249822 A1, 1974. (u) Rudolph, K. H.; Buechner, W.; Noll, W. Ger. Offen. D.E. 2308238 A1, 1974. (v) Atherton, J. H.; Plumb, J. B.; Stuart, R. S. G.B. Patent 1406356 A, 1975. (w) Chandra, G.; Griffiths, B. J.; Westall, S. Ger. Offen. D.E. 2607714 A1, 1976. (x) Fort, G. G.B. Patent 1473335 A, 1977. (y) Belyakova, Z. V.; Knyazeva, L. K.; Chernyshev, E. A. Zh. Obshch. Khim. 1978, 48, 1373. (z) 
amine, ${ }^{102 a c, 102 a e, 102 a g-102 a h, 104}$ or a chelating L-type ligand such as 1,5 -cyclooctadiene. ${ }^{105}$ Platinum(II) precatalysts are reduced in situ to the active catalyst by a similar mechanism to the activation of Speier's catalyst. ${ }^{1 \mathrm{1}, 106}$ Platinum(0) precatalysts typically bear stabilizing phosphine, ${ }^{107}$ olefin, ${ }^{108}$ or $N$-heterocyclic

Valles, E. M.; Macosko, C. W. Macromolecules 1979, 12, 521. (aa) Vybiral, V.; Svoboda, P.; Hetflejs, J. Collect. Czech. Chem. Commun. 1979, 44, 866. (ab) Kreis, G.; Wegehaupt, K. H. Ger. Offen. D.E. 2846621 A1, 1980. (ac) Weitemeyer, C.; Koerner, G. Ger. Offen. D.E. 2918254 A1, 1980. (ad) Kopylova, L. I.; Ivanova, N. D.; Voropaev, V. N.; Domnina, E. S.; Skvortsova, G. G.; Voronkov, M. G. Zh. Obshch. Khim. 1985, 55, 1036. (ae) Albinati, A.; Caseri, W. R.; Pregosin, P. S. Organometallics 1987, 6, 788. (af) Caseri, W.; Pregosin, P. S. Organometallics 1988, 7, 1373. (ag) Caseri, W.; Pregosin, P. S. J. Organomet. Chem. 1988, 356, 259. (ah) Skoda-Foldes, R.; Kollar, L.; Heil, B. J. Organomet. Chem. 1989, 366, 275. (ai) Coqueret, X.; Wegner, G. Organometallics 1991, 10, 3139. (aj) Friedmann, G.; Shreim, Y.; Brossas, J. Eur. Polym. J. 1992, 28, 271. (ak) Steffanut, P.; Osborn, J. A.; Decian, A.; Fisher, J. Chem. - Eur. J. 1998, 4, 2008. (al) Hopf, A.; Dotz, K. H. J. Mol. Catal. A: Chem. 2000, 164, 191.

${ }^{104}$ For representative examples, see: (a) Koerner, G. Ger. Offen. D.E. 1165028, 1964. (b) Chalk, A. J. U.S. Patent 3188300, 1965. (c) Flatt, D. V. U.S. Patent 3453233 A, 1969. (d) Yamamoto, K.; Hayashi, T.; Kumada, M. J. Am. Chem. Soc. 1971, 93, 5301. (e) Yamamoto, K.; Hayashi, T.; Kumada, M. J. Organometal. Chem. 1971, 28, C37. (f) Yamamoto, K.; Hayashi, T.; Kumada, M. J. Organometal. Chem. 1972, 46, C65. (g) Mink, A. E.; Mitchell, D. D. U.S. Patent 3944519 A, 1976. (h) Podol'skii, A. V.; Suvorov, A. L.; Cherezova, T. G.; Fridman, L. I. Zh. Obshch. Khim. 1977, 47, 1532. (i) Hafner, W.; Markl, E.; Eibel, L.; Kreis, G.; Samrowski, D.; Wick, M.; Deubzer, B.; Friedrich, W. Ger. Offen. D.E. 2809874 A1, 1979. (j) Kreis, G.; August, P. Ger. Offen. D.E. 2809875 A1, 1979. (k) Warrick, E. L.; Pierce, O. R.; Polmanteer, K. E.; Saam, J. C. Rubber Chem. Technol. 1979, 52, 437. (1) Legrow, G. E. U.S. Patent 4234713 A, 1980. (m) Brovko, V. S.; Skvortsov, N. K.; Reikhsfel'd, V. O. Zh. Obshch. Khim. 1981, $51,487$.

${ }^{105}$ For representative examples, see: (a) Knorre, H.; Rothe, W. Ger. Offen. D.E. 1271712, 1968. (b) Vahlensieck, H. J.; Seiler, C. D.; Koetzsch, H. J. Ger. Offen. D.E. 1937904 A, 1971. (c) Baney, R. H. U.S. Patent 3723497 A, 1973.

${ }^{106}$ For a mechanism study of (COD)PtR 2 activation, see: Jagadeesh, M. N.; Thiel, W.; Köhler, J.; Fehn, A. Organometallics 2002, 21, 2076.

${ }^{107}$ For representative examples, see: (a) Yamamoto, K.; Hayashi, T.; Kumada, M. J. Organometal. Chem. 1971, 28, C37. (b) Andrianov, K. A.; Soucek, J.; Hetflejs, J.; Khananashvili, L. M. Zh. Obshch. Khim. 1975, 45, 2215. (c) Reikhsfel'd, V. O.; Khvatova, T. P.; Astrakhanov, M. I. Zh. Obshch. Khim. 1977, 47, 2625. (d) Watanabe, H.; Asami, M.; Nagai, Y. J. Organomet. Chem. 1980, 195, 363. (e) Belyakova, Z. V.; Dmitriev, A. A.; Savushkina, V. T.; Bochkarev, V. N.; Chernishev, E. A. Zh. Obshch. Khim. 1985, 55, 627. (f) Zhang, X.; Lu, X.; Zhang, R.; Duan, H. Cuihua Xuebao 1986, 7, 378. (g) Marciniec, B.; Gulinski, J.; Urbaniak, W.; Nowicka, T.; Mirecki, J. Appl. Organomet. Chem. 1990, 4, 27.

${ }^{108}$ For representative examples, see: (a) Yamamoto, K.; Hayashi, T.; Kumada, M. J. Organometal. Chem. 1971, 28, C37. (b) Cavezzan, J. Fr. Patent F.R. 2575086 A1, 1986. (c) Chandra, G.; Yin, K. L. P. Eur. Pat. Appl. E.P. 173512 A2, 1986. (d) Chandra, G.; Lo, P. Y.; Hitchcock, P. B.; Lappert, M. F. Organometallics 1987, 6, 191. (e) Saruyama, T. Eur. Pat. Appl. E.P. 249944 A2, 1987. (f) Marciniec, B.; 
carbene (NHC) $)^{109,110}$ ligands. Platinum(0) precatalysts avoid the need for reduction in situ and are activated by simple exchange of a stabilizing ligand for a molecule of the unsaturated substrate.

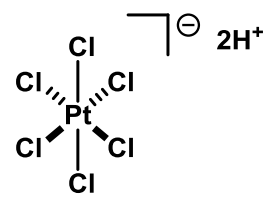

Speier's Catalyst, 1957

1

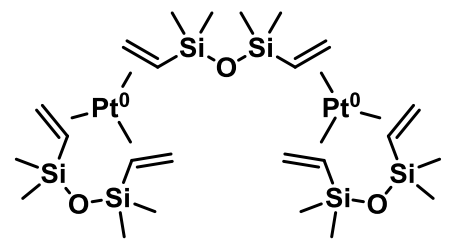

Karstedt's Catalyst, 1973

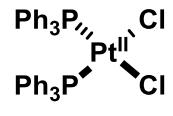

3

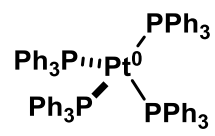

6

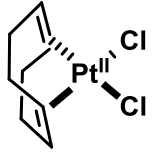

4

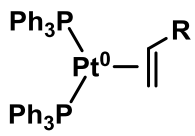

7

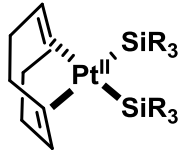

5

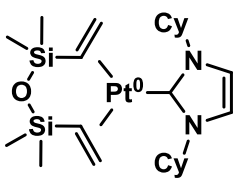

8

Figure 1. Typical platinum precatalysts for the hydrosilylation of olefins

Gulinski, J.; Urbaniak, W.; Nowicka, T.; Mirecki, J. Appl. Organomet. Chem. 1990, 4, 27. (g) Lewis, L. N.; Sumpter, C. A. J. Mol. Catal. A: Chem. 1996, 104, 293. (h) Steffanut, P.; Osborn, J. A.; Decian, A.; Fisher, J. Chem. - Eur. J. 1998, 4, 2008. (i) Huber, C.; Kokil, A.; Caseri, W. R.; Weder, C. Organometallics 2002, 21, 3817. (j) Sprengers, J. W.; De Greef, M.; Duin, M. A.; Elsevier, C. J. Eur. J. Inorg. Chem. 2003, 3811. (k) Yamamoto, Y.; Ohno, T.; Itoh, K. Organometallics 2003, 22, 2267. (1) Sprengers, J. W.; Agerbeek, M. J.; Elsevier, C. J.; Kooijman, H.; Spek, A. L. Organometallics 2004, 23, 3117.

${ }^{109}$ For reviews of $\operatorname{Pt}(0)$ complexes of NHC ligands in hydrosilylation, see: (a) Marko, I. E.; Sterin, S.; Buisine, O.; Mignani, G.; Branlard, P.; Tinant, B.; Declercq, J.-P. Science 2002, 298, 204. (b) BerthonGelloz, G.; Markó, I. E. In N-Heterocyclic Carbenes in Synthesis; Nolan, S. P., Ed.; Wiley-VCH: Weinheim, 2006; p. 119.

${ }^{110}$ For representative examples, see: (a) Chandra, G.; Lo, P. Y.; Hitchcock, P. B.; Lappert, M. F. Organometallics 1987, 6, 191. (b) Marko, I.; Sterin, S. W.O. Patent 2001042258 A1, 2001. (c) Buisine, O.; Marko, I.; Sterin, S. W.O. Patent 2002098888 A1, 2002. (d) Marko, I. E.; Sterin, S.; Buisine, O.; Berthon, G.; Michaud, G.; Tinant, B.; Declercq, J.-P. Adv. Synth. Catal. 2004, 346, 1429. (e) Buisine, O.; Berthon-Gelloz, G.; Briere, J.-F.; Sterin, S.; Mignani, G.; Branlard, P.; Tinant, B.; Declercq, J.-P.; Marko, I. E. Chem. Commun. 2005, 3856. (f) Berthon-Gelloz, G.; Buisine, O.; Briere, J.-F.; Michaud, G.; Sterin, S.; Mignani, G.; Tinant, B.; Declercq, J.-P.; Chapon, D.; Marko, I. E. J. Organomet. Chem. 2005, 690, 6156. (g) De Bo, G.; Berthon-Gelloz, G.; Tinant, B.; Markó, I. E. Organometallics 2006, 25, 1881. (h) Berthon-Gelloz, G.; Schumers, J.-M.; Lucaccioni, F.; Tinant, B.; Wouters, J.; Markó, I. E. Organometallics 2007, 26, 5731. (i) Larson, G. L.; Arkles, B. C.; Cameron, R. A. U.S. Patent 20100280266 A1, 2010. 
Today, the most industrially-relevant hydrosilylation catalyst is the olefin-stabilized $\operatorname{Pt}(0)$ complex developed by Karstedt in 1973 (2). ${ }^{1 b}$ Karstedt's catalyst (Figure 1) does not require in situ reduction, is more soluble than Speier's catalyst in vinylsiloxane polymers and in the alkyl silane products of hydrosilylation, and shows higher activity than Speier's catalyst in the hydrosilylation of olefins. ${ }^{1 j, 91,111}$ However, Karstedt's catalyst decomposes upon dissociation of the weakly-bound vinylsiloxane ligands to form platinum( 0 ) colloids, which reduces catalyst TON and increases the cost of hydrosilylation. ${ }^{1 \mathrm{i}, 1 \mathrm{j}, 112}$ L-type ligands such as phosphines and NHCs can protect against catalyst degradation and colloid formation, which increases the TON, but these $\sigma$-donating ligands dramatically reduce the TOF. ${ }^{113}$ Modification of the dienyl ligand (Figure 1) can increase the activity of the catalyst, but often does not protect against colloid formation. ${ }^{114}$ Induction periods are still observed using Karstedt's catalyst because the vinylsiloxane supporting ligands often bind more strongly to platinum than the unsaturated substrate. During the induction period, the supporting olefin ligands are removed by hydrosilylation or ligand exchange. ${ }^{112}$ Although transition metal catalysts bearing a wide variety of ligands have been reported in recent decades, Karstedt's catalyst is still the most general and widely used catalyst for the hydrosilylation of olefins. ${ }^{1,11}$

${ }^{111}$ Lewis, L. N.; Stein, J.; Gao, Y.; Colborn, R. E.; Hutchins, G. Platinum Met. Rev. 1997, 41, 66.

${ }^{112}$ Stein, J.; Lewis, L. N.; Gao, Y.; Scott, R. A. J. Am. Chem. Soc. 1999, 121, 3693.

${ }^{113}$ For representative examples of $\operatorname{Pt}(0)$ precatalysts supported by phosphine ligands, see: (a) Yamamoto, K.; Hayashi, T.; Kumada, M. J. Organometal. Chem. 1971, 28, C37. (b) Andrianov, K. A.; Soucek, J.; Hetflejs, J.; Khananashvili, L. M. Zh. Obshch. Khim. 1975, 45, 2215. (c) Reikhsfel'd, V. O.; Khvatova, T. P.; Astrakhanov, M. I. Zh. Obshch. Khim. 1977, 47, 2625. (d) Watanabe, H.; Asami, M.; Nagai, Y. J. Organomet. Chem. 1980, 195, 363. (e) Zhang, X.; Lu, X.; Zhang, R.; Duan, H. Cuihua Xuebao 1986, 7, 378. (f) Marciniec, B.; Gulinski, J.; Urbaniak, W.; Nowicka, T.; Mirecki, J. Appl. Organomet. Chem. 1990, 4, 27.

${ }^{114}$ For examples of $\operatorname{Pt}(0)$ alkene and alkyne precatalysts, see: (a) Chandra, G.; Lo, P. Y.; Hitchcock, P. B.; Lappert, M. F. Organometallics 1987, 6, 191. (b) Lewis, L. N.; Sumpter, C. A. J. Mol. Catal. A: Chem. 1996, 104, 293. (c) Steffanut, P.; Osborn, J. A.; Decian, A.; Fisher, J. Chem. - Eur. J. 1998, 4, 2008. (d) Huber, C.; Kokil, A.; Caseri, W. R.; Weder, C. Organometallics 2002, 21, 3817. (e) Sprengers, J. W.; De Greef, M.; Duin, M. A.; Elsevier, C. J. Eur. J. Inorg. Chem. 2003, 3811. (f) Yamamoto, Y.; Ohno, T.; Itoh, K. Organometallics 2003, 22, 2267. 


\subsubsection{Catalysis by Other Transition Metals}

Metal catalysts including noble metals such as palladium, rhodium, ruthenium, and iridium, terrestrially abundant metals like cobalt, nickel, and iron, electrophilic early transition metals, and lanthanides and actinides all show activity and unique patterns of selectivity in the hydrosilylation of multiple bonds. ${ }^{1}$ Despite the high cost of noble metals (Table 2), most industrial hydrosilylation processes still utilize noble metal catalysts for hydrosilylation because of their unsurpassed efficiency and long life. Platinum catalysts are the most commonly used for the hydrosilylation of olefins, ${ }^{1 \mathrm{j}}$ while rhodium catalysts are more efficient for alkyne $\mathrm{e}^{115}$ and carbonyl ${ }^{116}$ hydrosilylation, palladium is active in the hydrosilylation of conjugated dienes, ${ }^{117}$ and iridium is most selective in the hydrosilylation of allyl electrophiles. ${ }^{1 \mathrm{j}}$

${ }^{115}$ For examples, see: (a) Ojima, I.; Kumagai, M.; Nagai, Y. J. Organometal. Chem. 1974, 66, C14. (b) Watanabe, H.; Kitahara, T.; Motegi, T.; Nagai, Y. J. Organomet. Chem. 1977, 139, 215. (c) Brady, K. A.; Nile, T. A. J. Organomet. Chem. 1981, 206, 299. (d) Lappert, M. F.; Maskell, R. K. J. Organomet. Chem. 1984, 264, 217. (e) Takeuchi, R.; Tanouchi, N. J. Chem. Soc., Perkin Trans. 1 1994, 2909. (f) Takeuchi, R.; Nitta, S.; Watanabe, D. J. Org. Chem. 1995, 60, 3045. (g) Muraoka, T.; Matsuda, I.; Itoh, K. Tetrahedron Lett. 1998, 39, 7325. (h) Faller, J. W.; D'alliessi, D. G. Organometallics 2002, 21, 1743.

${ }^{116}$ For representative examples, see: (a) Ojima, I.; Nihonyanagi, M.; Nagai, Y. Chem. Commun. 1972, 938. (b) Nagata, Y.; Dohmaru, T.; Tsurugi, J. J. Org. Chem. 1973, 38, 795. (c) Benes, J.; Hetflejs, J. Collect. Czech. Chem. Commun. 1976, 41, 2264. (d) Hayashi, T.; Yamamoto, K.; Kasuga, K.; Omizu, H.; Kumada, M. J. Organomet. Chem. 1976, 113, 127. (e) Ojima, I.; Kogure, T.; Kumagai, M.; Horiuchi, S.; Sato, T. J. Organomet. Chem. 1976, 122, 83. (f) Brunner, H.; Reiter, B.; Riepl, G. Chem. Ber. 1984, 117 , 1330. (g) Brunner, H.; Fisch, H. J. Organomet. Chem. 1987, 335, 15.

117 For reviews discussing the utility of Pd in hydrosilylation see: (a) Marciniec, B.; Gulinski, J.; Urbaniak, W.; Kornetka, Z. W.; Pergamon Press: Oxford, 1992; Ch. (b) Roy, A. K. Adv. Organomet. Chem. 2008, 55, 12. 
Table 2. Average Monthly Price of Precious Metals (US\$/troy oz.) ${ }^{118}$

\begin{tabular}{cc} 
Metal & Average Price \\
\hline Platinum & $\$ 870.69$ \\
Palladium & $\$ 358.75$ \\
Rhodium & $\$ 1823.98$ \\
Iridium & $\$ 384.34$ \\
Ruthenium & $\$ 115.75$
\end{tabular}

After platinum, rhodium is the most common metal used in hydrosilylation catalysts, despite its even higher average cost (Table 2). Rhodium catalysts, while acceptable catalysts for the hydrosilylation of olefins, ${ }^{119}$ surpass the efficiency and selectivity of platinum catalysts in the hydrosilylation of alkynes to form cis- or trans-alkenylsilanes (Eq. 5). ${ }^{115}$ Rhodium catalysts are also excellent for the hydrosilylation of aldehydes and ketones (Eq. 6). ${ }^{116}$ In addition, chiral rhodium catalysts of phosphorus- ${ }^{120}$ and nitrogenbased $^{121}$ chelating ligands provide high levels of enantioinduction and are used to synthesize chiral

118 Data are based on Johnson Matthey monthly average prices, obtained from: http://www.platinum.matthey.com/pgm/prices/, March 15, 2014.

${ }^{119}$ For representative examples, see: (a) Millan, A.; Fernandez, M. J.; Bentz, P.; Maitlis, P. M. J. Mol. Catal. 1984, 26, 89. (b) Baruah, J. B.; Osakada, K.; Yamamoto, T. J. Mol. Catal. A: Chem. 1995, 101, 17. (c) Ganicz, T.; Mizerska, U.; Moszner, M.; O'brien, M.; Perry, R.; Stanczyk, W. A. Appl. Catal., A 2004, 259, 49. (d) Rivera, G.; Crabtree, R. H. J. Mol. Catal. A: Chem. 2004, 222, 59.

${ }^{120}$ For representative examples, see: (a) Hayashi, T.; Yamamoto, K.; Kumada, M. Tetrahedron Lett. 1974, 4405. (b) Corriu, R.; Moreau, J. J. E. J. Organomet. Chem. 1975, 85, 19. (c) Corriu, R. J. P.; Moreau, J. J. E. J. Organomet. Chem. 1975, 91, C27. (d) Johnson, T. H.; Klein, K. C.; Thomen, S. J. Mol. Catal. 1981, 12, 37. (e) Brunner, H.; Knott, A. Z. Naturforsch., B: Anorg. Chem., Org. Chem. 1985, 40B, 1243. (f) Karim, A.; Mortreux, A.; Petit, F. Tetrahedron Lett. 1986, 27, 345. (g) Gondos, G.; Gera, L.; Bartok, M.; Orr, J. C. J. Organomet. Chem. 1989, 373, 365.

${ }^{121}$ For representative examples, see: (a) Payne, N. C.; Stephan, D. W. Inorg. Chem. 1982, 21, 182. (b) Brunner, H.; Becker, R.; Riepl, G. Organometallics 1984, 3, 1354. (c) Brunner, H.; Weber, H. Chem. Ber. 1985, 118, 3380. (d) Brunner, H.; Kuerzinger, A. J. Organomet. Chem. 1988, 346, 413. (e) Botteghi, C.; Schionato, A.; Chelucci, G.; Brunner, H.; Kuerzinger, A.; Obermann, U. J. Organomet. Chem. 1989, 370, 17. (f) Nishiyama, H.; Sakaguchi, H.; Nakamura, T.; Horihata, M.; Kondo, M.; Itoh, K. Organometallics 1989, 8,846 . 
secondary alcohols. ${ }^{122}$ Typically, rhodium hydrosilylation catalysts are complexes of $\sigma$-donating ligands in the $+\mathrm{I}$ or + III oxidation state and the modified Chalk-Harrod cycle utilizing the $\mathrm{Rh}(\mathrm{I} / \mathrm{III})$ redox couple is generally accepted as the mechanism of hydrosilylation catalyzed by rhodium complexes (see section 1.2.3, Scheme 11). ${ }^{138,143}$
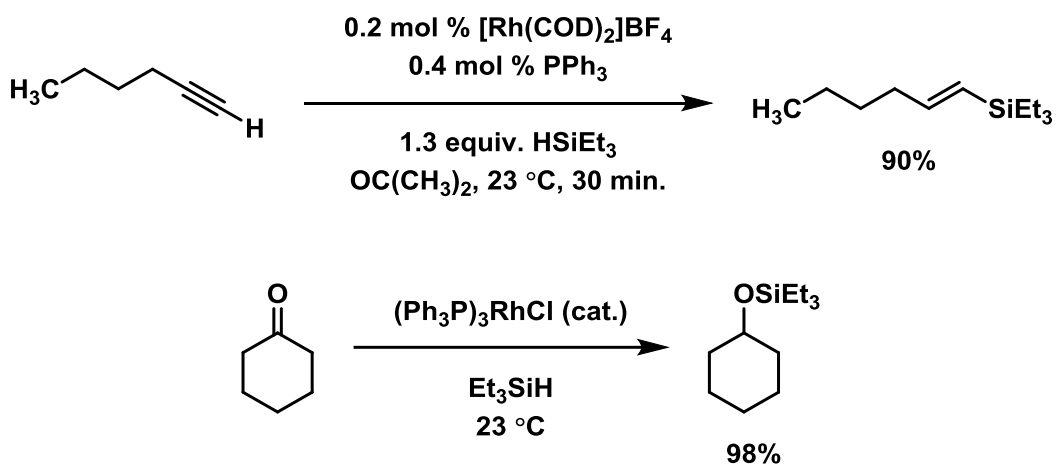

(Eq. 6)

Palladium complexes are poor catalysts for the hydrosilylation of olefins, ${ }^{123}$ which can be attributed to the facile reduction of Pd(II) complexes to Pd metal by hydrosilanes. ${ }^{117}$ Dienes, however, are better ligands for palladium( 0 ) complexes and are good substrates for palladium-catalyzed hydrosilylation (Eq. 7, see section 1.3). ${ }^{2 \mathrm{~d}, 2 \mathrm{e}, 124}$ Palladium hydrosilylation precatalysts are often $\mathrm{Pd}(\mathrm{II})$ complexes of

${ }^{122}$ For reviews of rhodium-catalyzed asymmetric hydrosilylation, see: (a) Kagan, H. B. Pure Appl. Chem. 1975, 43, 401. (b) Brunner, H. Angew. Chem. 1983, 95, 921. (c) Brunner, H.; Reiter, B.; Riepl, G. Chem. Ber. Recl. 1984, 117, 1330. (d) Ojima, I. Pure Appl. Chem. 1984, 56, 99. (e) Blystone, S. L. Chem. Rev. 1989, 89, 1663.

${ }^{123}$ For representative examples, see: (a) Gulinski, J.; James, B. R. J. Mol. Catal. 1992, 72, 167. (b) Widenhoefer, R. A.; Vadehra, A. Tetrahedron Lett. 1999, 40, 8499. (c) Widenhoefer, R. A. Acc. Chem. Res. 2002, 35, 905.

${ }^{124}$ For representative examples, see: (a) Hayashi, T.; Kabeta, K.; Yamamoto, T.; Tamao, K.; Kumada, M. Tetrahedron Lett. 1983, 24, 5661. (b) Hayashi, T.; Kabeta, K. Tetrahedron Lett. 1985, 26, 3023. (c) Hayashi, T.; Hengrasmee, S.; Matsumoto, Y. Chem. Lett. 1990, 1377. (d) Gulinski, J.; James, B. R. J. Mol. Catal. 1992, 72, 167. (e) Hatanaka, Y.; Goda, K.-I.; Yamashita, F.; Hiyama, T. Tetrahedron Lett. 1994, 35, 7981. 
phosphine or nitrogen-based ligands and may proceed via either the Chalk-Harrod or modified ChalkHarrod mechanism. ${ }^{125}$

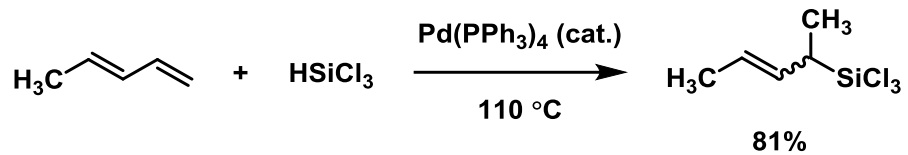

First-row transition metals are known to catalyze hydrosilylation of a variety of olefins and other carbon-carbon multiple bonds. Most, however, do so with lower TON and/or TOF than the noble metal catalysts. Notable examples of first-row transition metal catalyzed hydrosilylation include the hydrosilylation of styrenes and olefins with bisiminopyridine iron complexes (Eq. 8). ${ }^{87 \mathrm{~b}}$ Well-defined, low-valent iron complexes supported by redox-active ligands also catalyze the hydrosilylation of conjugated dienes (see sections 1.3.3 and 1.3.4). ${ }^{2 \mathrm{~m}}$

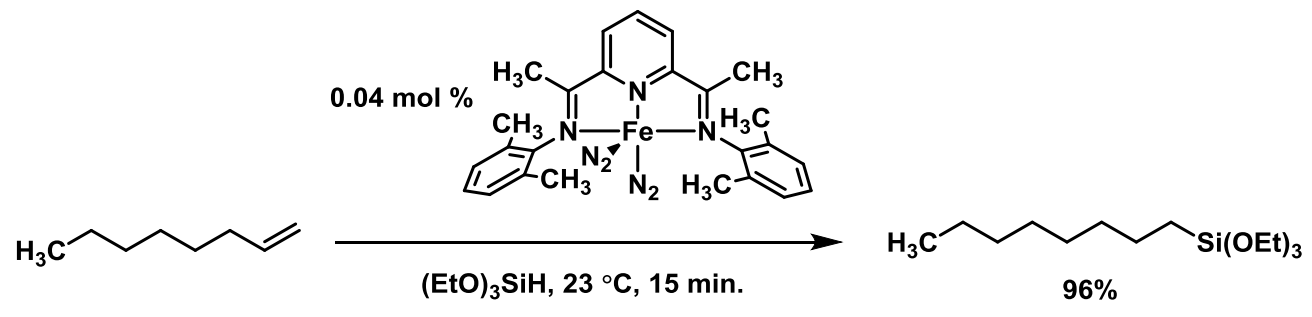

(Eq. 8)

Early, electrophilic transition metal complexes, as well as lanthanide and actinide metals, are known to catalyze hydrosilylation through a different mechanism than late transition metal catalysts (see section 1.2.3, Scheme 12). Early transition metals can catalyze the addition of primary silanes to ketones, olefins, and alkynes, but are not as active with more practical tertiary silanes (Eq. 9). ${ }^{88 \mathrm{~b}}$

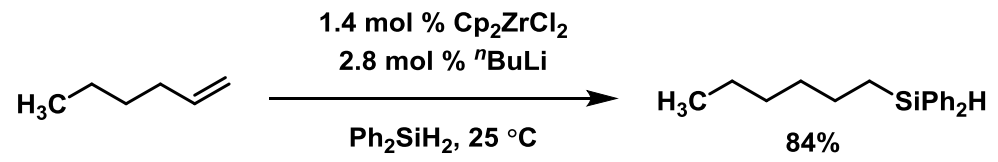

(Eq. 9)

125 For mechanistic studies of Pd-catalyzed hydrosilylation, see: (a) Lapointe, A. M.; Rix, F. C.; Brookhart, M. J. Am. Chem. Soc. 1997, 119, 906. (b) Magistrato, A.; Woo, T. K.; Togni, A.; Rothlisberger, U. Organometallics 2004, 23, 3218. 


\subsubsection{Mechanism}

The study of mechanism in transition metal-catalyzed hydrosilylation is riddled with challenges. Catalyst loadings are typically extremely low, in the ppm or ppb range with respect to substrates, and catalytic intermediates are highly reactive, short-lived, and unstable. In addition, the turnover-limiting step for many catalysts is ligand dissociation prior to the interaction of the catalyst with substrate, which limits use of kinetic analysis to study the elementary steps of the catalytic cycle. ${ }^{\text {h }}$ Despite these challenges, the broad utility of olefin hydrosilylation has motivated a number of investigations into hydrosilylation mechanisms. Today, several mechanisms at different types of transition metal catalysts are widely accepted. Platinum complexes catalyze hydrosilylation by the Chalk-Harrod mechanism, Rhodium catalysts proceed by a modified version of this mechanism, Ruthenium and Palladium can proceed by either one, and $d^{0}$ catalysts by a mechanism involving $\sigma$-bond metathesis.

In 1965, Chalk and Harrod proposed a mechanism for platinum-catalyzed hydrosilylation based on similarities they observed to homogeneous hydrogenation reactions (Scheme 8). ${ }^{126}$ The Chalk-Harrod mechanism was proposed based on work with Speier's catalyst, but is still widely accepted as the mechanism of hydrosilylation using most platinum precatalysts. ${ }^{1}$ In the Chalk-Harrod mechanism, lowvalent complex A oxidatively adds the relatively weak $\mathrm{Si}-\mathrm{H}$ bond to form silyl hydride complex $\mathbf{B}$. Migratory insertion of the coordinated olefin into the $\mathrm{Pt}-\mathrm{H}$ bond forms alkyl complex $\mathbf{C}$, which is poised to reductively eliminate the alkyl silane product and regenerate complex $\mathbf{A}$. The Chalk-Harrod mechanism explains many of the authors' and previous researchers' observations about the selectivity and rate of hydrosilylation using various substrates. ${ }^{1,91,126}$ Two key questions they considered are whether the cycle proceeds via a $\mathrm{Pt}(0 / \mathrm{II})$ cycle or a $\mathrm{Pt}(\mathrm{II} / \mathrm{IV})$ cycle and whether insertion of the olefin occurs first into the $\mathrm{Pt}-\mathrm{H}$ or Pt-Si bond of intermediate $\mathbf{C}$.

${ }^{126}$ Chalk, A. J.; Harrod, J. F. J. Am. Chem. Soc. 1965, 87, 16. 
Chalk and Harrod postulated that reduction of hexachloroplatinic acid to the tetrachloroplatinum(II) dianion activated the precatalyst and proposed a $\mathrm{Pt}(\mathrm{II} / \mathrm{IV})$ catalytic cycle. In the $\mathrm{Pt}(\mathrm{II} / \mathrm{IV})$ cycle, it was thought that the purpose of catalyst activation was to reduce the number of chloride ligands on the precatalyst to a $1: 1 \mathrm{Pt}: \mathrm{Cl}$ ratio. ${ }^{126}$ Since this original proposal, careful study of a number of $\mathrm{Pt}(0), \mathrm{Pt}(\mathrm{II})$, and $\mathrm{Pt}(\mathrm{IV})$ precatalysts has established the existence of both $\mathrm{Pt}(0 / \mathrm{II})^{127}$ and $\mathrm{Pt}(\mathrm{II} / \mathrm{IV})^{126,128}$ catalytic cycles using different precatalysts. ${ }^{1 \mathrm{~h}}$

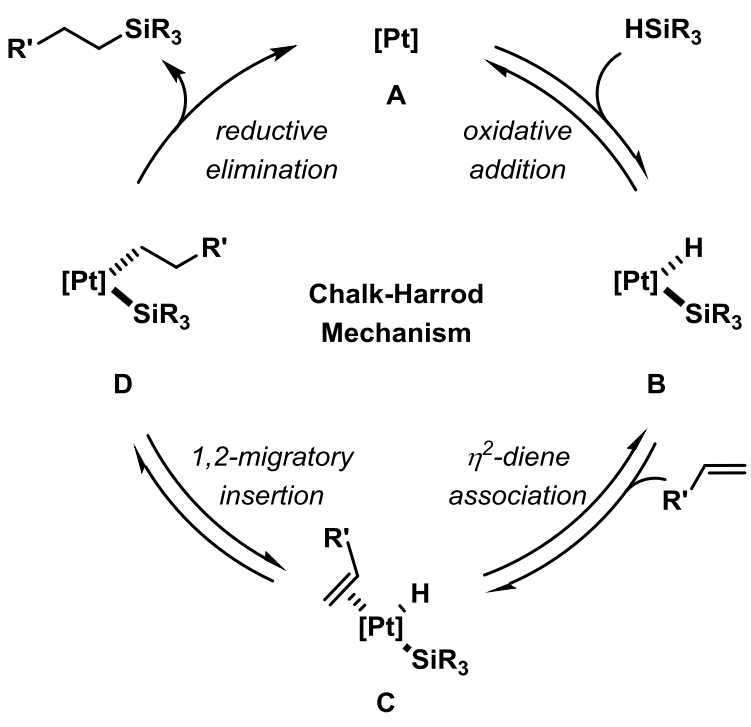

\section{Scheme 8. Chalk-Harrod Mechanism of Olefin Hydrosilylation}

Insertion of the coordinated olefin into the $\mathrm{Pt}-\mathrm{H}$ bond of $\mathbf{C}$ rather than the $\mathrm{Pt}-\mathrm{Si}$ bond can be inferred from several experimental observations and has been supported by theoretical studies. ${ }^{127}$ In most hydrosilylation reactions catalyzed by Speier's and Karstedt's catalysts, hydrosilylation generates

${ }^{127}$ For a detailed theoretical discussion of $\mathrm{Pt}(0 / \mathrm{II})$ hydrosilylation, see (a) Sakaki, S.; Ogawa, M.; Musashi, Y.; Arai, T. J. Am. Chem. Soc. 1994, 116, 7258. (b) Ozawa, F.; Hikida, T.; Hayashi, T. J. Am. Chem. Soc. 1994, 116, 2844. (c) Sakaki, S.; Mizoe, N.; Sugimoto, M. Organometallics 1998, 17, 2510. (d) Sakaki, S.; Mizoe, N.; Musashi, Y.; Biswas, B.; Sugimoto, M. J. Phys. Chem. A 1998, 102, 8027. (e) Sakaki, S.; Mizoe, N.; Musashi, Y.; Sugimoto, M. J. Mol. Struct.: THEOCHEM 1999, 461-462, 533. (f) Sakaki, S.; Mizoe, N.; Sugimoto, M.; Musashi, Y. Coord. Chem. Rev. 1999, 190-192, 933. (g) Giorgi, G.; De Angelis, F.; Re, N.; Sgamellotti, A. J. Mol. Struct.: THEOCHEM 2003, 623, 277.

${ }^{128}$ Roy, A. K.; Taylor, R. B. J. Am. Chem. Soc. 2002, 124, 9510. 
exclusively the product of anti-Markovnikov addition. ${ }^{1}$ To form the anti-Markovnikov product, insertion of the olefin into the Pt-H bond would generate a terminally-bound alkyl complex (D), whereas insertion into the Pt-Si bond would form an intermediate in which Pt is bound to a more sterically hindered carbon ligand. Additionally, generation of 1-silylalkane products in reactions of internal olefins suggested a metal hydride intermediate that reacts rapidly and reversibly by migratory insertion and $\beta$-hydride elimination to form the most stable metal-alkyl complex. ${ }^{1 a, 91}$ Isotope labeling studies show rapid hydrogen/deuterium scrambling into the olefin substrate, which provides further support for the rapid and reversible migratory insertion of olefins into the $\mathrm{Pt}-\mathrm{H}$ bond prior to reductive elimination. ${ }^{90 \mathrm{~b}}$

The intermediates on the Chalk-Harrod cycle are reactive and short-lived, and although a wide variety of stable platinum complexes can be isolated and characterized, isolation of intermediates on the Chalk-Harrod cycle is difficult (Figure 2). Complexes of type A in the Chalk-Harrod cycle (Scheme 8) are still not well-defined. Although many catalytically active $\operatorname{Pt}(0)$ complexes are reported that resemble the $\operatorname{Pt}(0)$ intermediate on the Chalk-Harrod cycle, ${ }^{102-108}$ all show induction periods in hydrosilylation reactions that exclude these complexes as true catalytic intermediates. ${ }^{128}$ In contrast, numerous examples of complexes of type B have been isolated and characterized (Figure 2), including a variety of complexes bearing phosphine ligands $(\mathbf{9}, \mathbf{1 0}),{ }^{129,130}$ a series of complexes of $\eta^{5}$-cyclopentadienyl ligands $(\mathbf{1 1}),{ }^{131}$ and

${ }^{129}$ For a review of transition-metal silyl complexes, see: Corey, J. Y.; Braddock-Wilking, J. Chem. Rev. 1998, 99, 175.

${ }^{130}$ For examples of $\mathrm{L}_{\mathrm{n}} \mathrm{Pt}(\mathrm{H})\left(\mathrm{SiR}_{3}\right)_{\mathrm{m}}$ complexes, see (a) Ebsworth, E. a. V.; Marganian, V. M.; Reed, F. J. S.; Gould, R. O. J. Chem. Soc., Dalton Trans. 1978, 1167. (b) Paonessa, R. S.; Prignano, A. L.; Trogler, W. C. Organometallics 1985, 4, 647. (c) Packett, D. L.; Syed, A.; Trogler, W. C. Organometallics 1988, 7, 159. (d) Mullica, D. F.; Sappenfield, E. L.; Hampden-Smith, M. J. Polyhedron 1991, 10, 867. (e) Heyn, R. H.; Tilley, T. D. J. Am. Chem. Soc. 1992, 114, 1917. (f) Grumbine, S. D.; Tilley, T. D.; Arnold, F. P.; Rheingold, A. L. J. Am. Chem. Soc. 1993, 115, 7884. (g) Abdol Latif, L.; Eaborn, C.; Pidcock, A. P.; Ng, S. W. J. Organomet. Chem. 1994, 474, 217. (h) Shimada, S.; Tanaka, M.; Honda, K. J. Am. Chem. Soc. 1995, $117,8289$.

${ }^{131}$ Boardman, L. D. Organometallics 1992, 11, 4194. 
a few examples with nitrogen-based $\sigma$-donor ligands $(\mathbf{1 2}, \mathbf{1 3}) \cdot{ }^{132}$ Although a few examples of iridium and rhodium analogs of intermediate $\mathbf{C}$ have been reported, ${ }^{133}$ platinum analogs are not known. A select few complexes of type D bearing phosphine ancillary ligands have been reported in the context of hydrosilylation. ${ }^{127 \mathrm{~b}, 134}$

In 2002, Roy and Taylor synthesized the first examples of characterized platinum (II) complexes bearing both olefin and silyl ligands $\left(\mathbf{1 4}, \mathbf{1 5}\right.$, Figure 2). ${ }^{128}$ Hydrosilylation catalyzed by platinum complex $\mathbf{1 4}$ does not exhibit an induction period, which suggests that $\mathbf{1 4}$ may be an intermediate on the catalytic cycle. In addition, complex $\mathbf{1 4}$ is observed unchanged during catalysis. The authors conclude that $\mathbf{1 4}$ is likely to be the resting state of the catalyst. Complex $\mathbf{1 4}$ could be of type $\mathbf{C}$ or $\mathbf{A}$ in the Chalk-Harrod cycle (Scheme 8), depending on the redox couple employed in the catalytic cycle. Roy and Taylor showed that complexes 14 and 15 did not undergo migratory insertion of the coordinated COD or other olefins into the $\mathrm{Pt}-\mathrm{Si}$ bond. Because $\mathbf{1 4}$ is the resting state and migratory insertion is not facile at $\mathbf{1 4}$, oxidative addition must be the first step in the catalytic cycle, which implicates a $\mathrm{Pt}(\mathrm{II}) / \mathrm{Pt}(\mathrm{IV})$ mechanism. The existence and reactions of $\mathbf{1 4}$ and $\mathbf{1 5}$ also provide the first experimental evidence that migratory insertion of olefin ligands into $\mathrm{Pt}-\mathrm{Si}$ bonds is not facile. ${ }^{128}$

132 For a complex supported by a Tp' ligand, see: (a) Reinartz, S.; White, P. S.; Brookhart, M.; Templeton, J. L. Organometallics 2000, 19, 3748. For a complex supported by a bisimine ligand, see: (b) Fang; Scott, B. L.; Watkin, J. G.; Kubas, G. J. Organometallics 2000, 19, 4193.

${ }^{133}$ For examples, see: (a) Auburn, M. J.; Stobart, S. R. Inorg. Chem. 1985, 24, 318. (b) Fernandez, M. J.; Esteruelas, M. A.; Jimenez, M. S.; Oro, L. A. Organometallics 1986, 5, 1519. (c) Fernandez, M. J.; Esteruelas, M. A.; Oro, L. A.; Apreda, M. C.; Foces-Foces, C.; Cano, F. H. Organometallics 1987, 6, 1751. (d) Ruiz, J.; Bentz, P. O.; Mann, B. E.; Spencer, C. M.; Taylor, B. F.; Maitlis, P. M. J. Chem. Soc., Dalton Trans. 1987, 2709. (e) Duckett, S. B.; Haddleton, D. M.; Jackson, S. A.; Perutz, R. N.; Poliakoff, M.; Upmacis, R. K. Organometallics 1988, 7, 1526. (f) Fernandez, M. J.; Esteruelas, M. A.; Covarrubias, M.; Oro, L. A.; Apreda, M. C.; Foces-Foces, C.; Cano, F. H. Organometallics 1989, 8, 1158. (g) Tanke, R. S.; Crabtree, R. H. Organometallics 1991, 10, 415. (h) Duckett, S. B.; Perutz, R. N. Organometallics 1992, 11, 90. (i) Duczmal, W.; Maciejewska, B.; Sliwinska, E.; Marciniec, B. Transition Met. Chem. 1995, 20, 162. (j) Auburn, M. J.; Holmes-Smith, R. D.; Stobart, S. R.; Bakshi, P. K.; Cameron, T. S. Organometallics 1996, 15, 3032. (k) Marciniec, B.; Krzyzanowski, P.; Kubicki, M. Polyhedron 1996, 15, 4233. (1) Carlton, L.; Molapisi, J. J. J. Organomet. Chem. 2000, 609, 60.

${ }^{134}$ Ozawa, F. J. Organomet. Chem. 2000, 611, 332. 


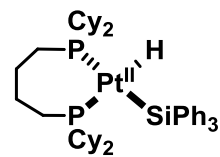

9

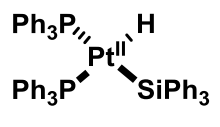

10

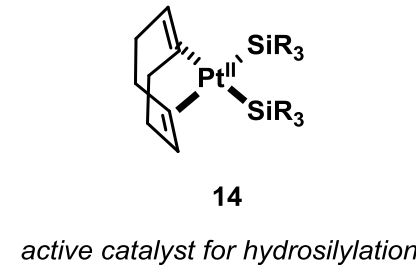

14

active catalyst for hydrosilylation

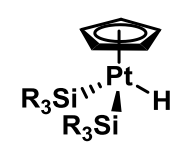

11

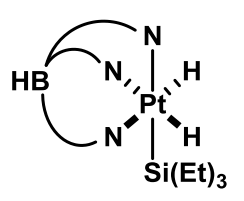

12

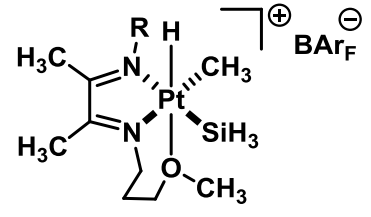

13

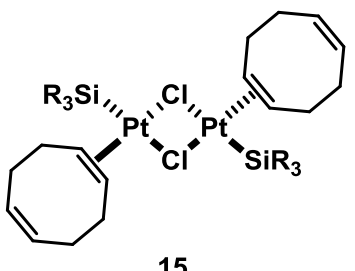

15

Figure 2. Synthetic Analogs of Intermediates on the Chalk-Harrod Cycle

A number of theoretical studies of platinum-catalyzed hydrosilylation have confirmed and expanded on the proposed Chalk-Harrod mechanism (Scheme 9). ${ }^{127}$ Oxidative addition of $\mathrm{Si}-\mathrm{H}$ to $\mathrm{Pt}(0)$ has been shown to proceed through a planar transition state that is unlike the asymmetric transition state for $\mathrm{C}-\mathrm{Si}$ reductive elimination. ${ }^{127 \mathrm{e}}$ Reductive elimination was predicted to be accelerated by addition of a second equivalent of olefin, which is attributed to transition state stabilization from back-donation into the $\pi^{*}$ orbital of the olefin ligand. Although reductive elimination has often been assumed to be turnoverlimiting, ${ }^{135}$ recent computational results argue that the highest energy barrier may be for a rotation of the alkyl ligand preceding reductive elimination rather than reductive elimination itself. ${ }^{136}$

${ }^{135}$ Tsipis, C. A.; Kefalidis, C. E. J. Organomet. Chem. 2007, 692, 5245.

${ }^{136}$ Tsipis, C. A.; Kefalidis, C. E. Organometallics 2006, 25, 1696. 


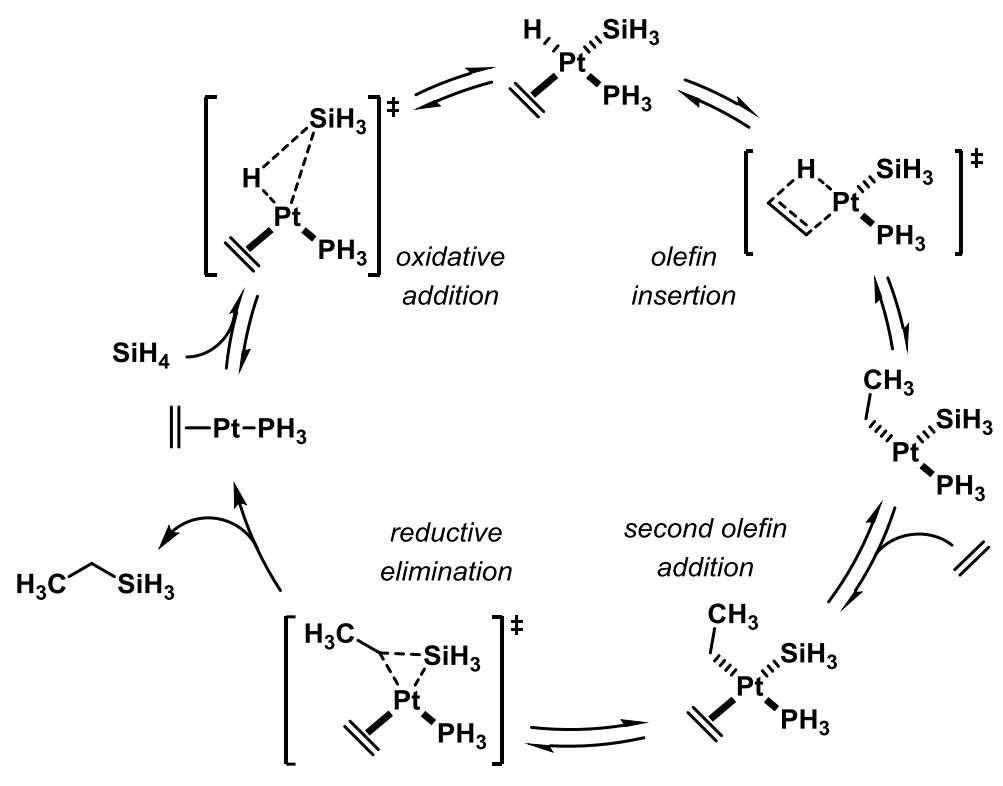

\section{Scheme 9. Expanded Chalk-Harrod Mechanism}

Transition metal-catalyzed hydrosilylation is often accompanied by side-reactions such as olefin isomerization, oligomerization, polymerization, hydrogenation, and dehydrogenative silylation, as well as the dehydrogenative oligomerization of silyl hydrides. ${ }^{\text {lh }}$ The types of side-products observed depend on the identity of the catalyst and substrates as well as the reaction conditions. Catalysis by the Chalk-Harrod mechanism cannot explain all of these byproducts. One that has been especially problematic to rationalize is the formation of vinylsilanes, often observed when reactions are catalyzed by metals other than platinum. ${ }^{137}$

${ }^{137}$ (a) Schroeder, M. A.; Wrighton, M. S. J. Organomet. Chem. 1977, 128, 345. (b) Mitchener, J. C.; Wrighton, M. S. J. Am. Chem. Soc. 1981, 103, 975. 


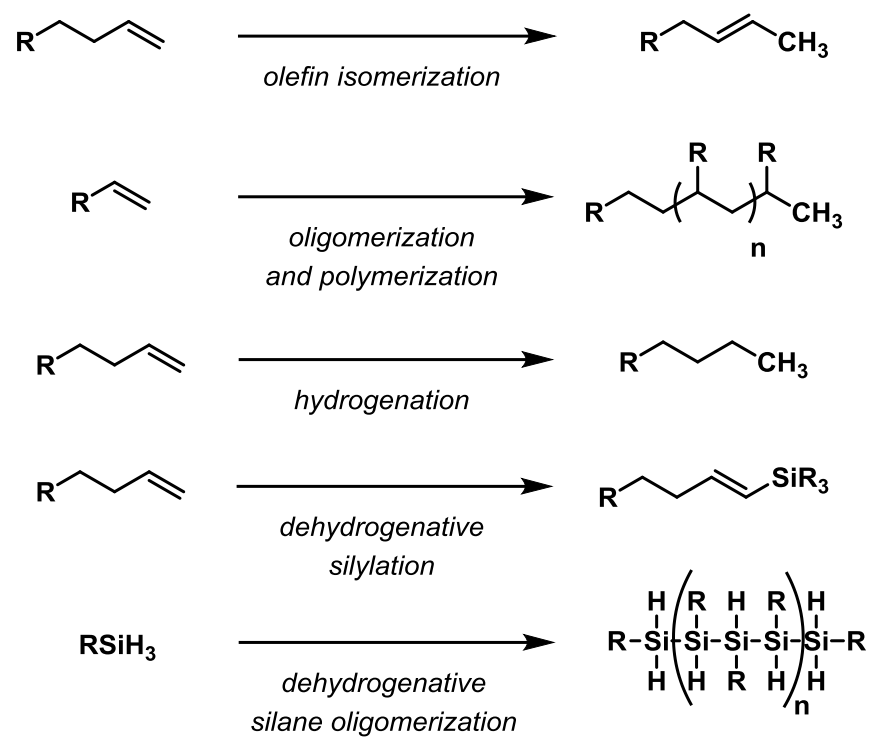

\section{Scheme 10. Common Side-Products of Transition Metal-Catalyzed Hydrosilylation}

The modified Chalk-Harrod mechanism was proposed in 1977 by Wrighton et al. to explain the formation of vinylsilanes in hydrosilylation catalyzed by $\mathrm{Fe}(\mathrm{CO})_{5} \cdot{ }^{137}$ Wrighton et al postulated that migratory insertion into the $\mathrm{M}-\mathrm{Si}$ bond could be faster than migratory insertion into $\mathrm{M}-\mathrm{H}$ ("Modified Chalk-Harrod Mechanism", Scheme 11). Duckett et al. later suggested that the M-H bond could be removed in catalyst activation and M-Si migratory insertion could proceed in the absence of a hydride ligand on the metal ("Two-Silicon Mechanism", Scheme 11). ${ }^{133 \mathrm{~h}}$ Subsequent studies of rhodium-, ${ }^{138}$ iridium-, ${ }^{139}$ cobalt-, ${ }^{140}$ and iron-catalyzed ${ }^{141}$ hydrosilylation reactions have supported Wrighton and

138 (a) Millan, A.; Towns, E.; Maitlis, P. M. Chem. Commun. 1981, 673. (b) Onopchenko, A.; Sabourin, E. T.; Beach, D. L. J. Org. Chem. 1983, 48, 5101. (c) Millan, A.; Fernandez, M. J.; Bentz, P.; Maitlis, P. M. J. Mol. Catal. 1984, 26, 89. (d) Onopchenko, A.; Sabourin, E. T.; Beach, D. L. J. Org. Chem. 1984, 49, 3389. (e) Ojima, I.; Fuchikami, T.; Yatabe, M. J. Organomet. Chem. 1984, 260, 335. (f) Ojima, I.; Clos, N.; Donovan, R. J.; Ingallina, P. Organometallics 1990, 9, 3127. (g) Bergens, S. H.; Noheda, P.; Whelan, J.; Bosnich, B. J. Am. Chem. Soc. 1992, 114, 2128.

${ }^{139}$ Oro, L. A.; Fernandez, M. J.; Esteruelas, M. A.; Jimenez, M. S. J. Mol. Catal. 1986, 37, 151.

${ }^{140}$ (a) Reichel, C. L.; Wrighton, M. S. Inorg. Chem. 1980, 19, 3858. (b) Seitz, F.; Wrighton, M. S. Angew. Chem. 1988, 100, 281. (c) Brookhart, M.; Grant, B. E. J. Am. Chem. Soc. 1993, 115, 2151. (d) Chatani, N.; Kodama, T.; Kajikawa, Y.; Murakami, H.; Kakiuchi, F.; Ikeda, S.-I.; Murai, S. Chem. Lett. 2000, 14. 
Duckett's mechanism proposals. Notably, catalysts based on ruthenium have been reported that can undergo both the Chalk-Harrod and the modified Chalk-Harrod mechanisms depending on the steric environment created by the supporting ligands. ${ }^{142}$

A computational study of ethylene hydrosilylation by $\left[\mathrm{RhCl}\left(\mathrm{PH}_{3}\right)_{3}\right]$ predicts that the modified Chalk-Harrod mechanism is preferred over the Chalk-Harrod mechanism. By comparing the activation barriers for each elementary step in the two mechanisms, the authors found that the $\mathrm{C}-\mathrm{Si}$ reductive elimination step in the Chalk-Harrod mechanism is predicted to exhibit a higher energy barrier than either of the two potentially turnover-limiting steps in the modified Chalk-Harrod mechanism, olefin insertion into $\mathrm{Rh}-\mathrm{Si}$ or oxidative addition of $\mathrm{H}-\mathrm{Si}^{143}$ Despite the elegant mechanism work described in these studies, there is no fundamental understanding of the reasons why platinum catalysts proceed via the Chalk-Harrod mechanism and other late transition metals by a modified Chalk-Harrod mechanism. ${ }^{143}$

141 (a) Mitchener, J. C.; Wrighton, M. S. J. Am. Chem. Soc. 1981, 103, 975. (b) Randolph, C. L.; Wrighton, M. S. J. Am. Chem. Soc. 1986, 108, 3366.

142 (a) Maruyama, Y.; Yamamura, K.; Nakayama, I.; Yoshiuchi, K.; Ozawa, F. J. Am. Chem. Soc. 1998, 120, 1421. (b) Maruyama, Y.; Yamamura, K.; Sagawa, T.; Katayama, H.; Ozawa, F. Organometallics 2000, 19, 1308.

${ }^{143}$ Sakaki, S.; Sumimoto, M.; Fukuhara, M.; Sugimoto, M.; Fujimoto, H.; Matsuzaki, S. Organometallics 2002, 21, 3788 . 

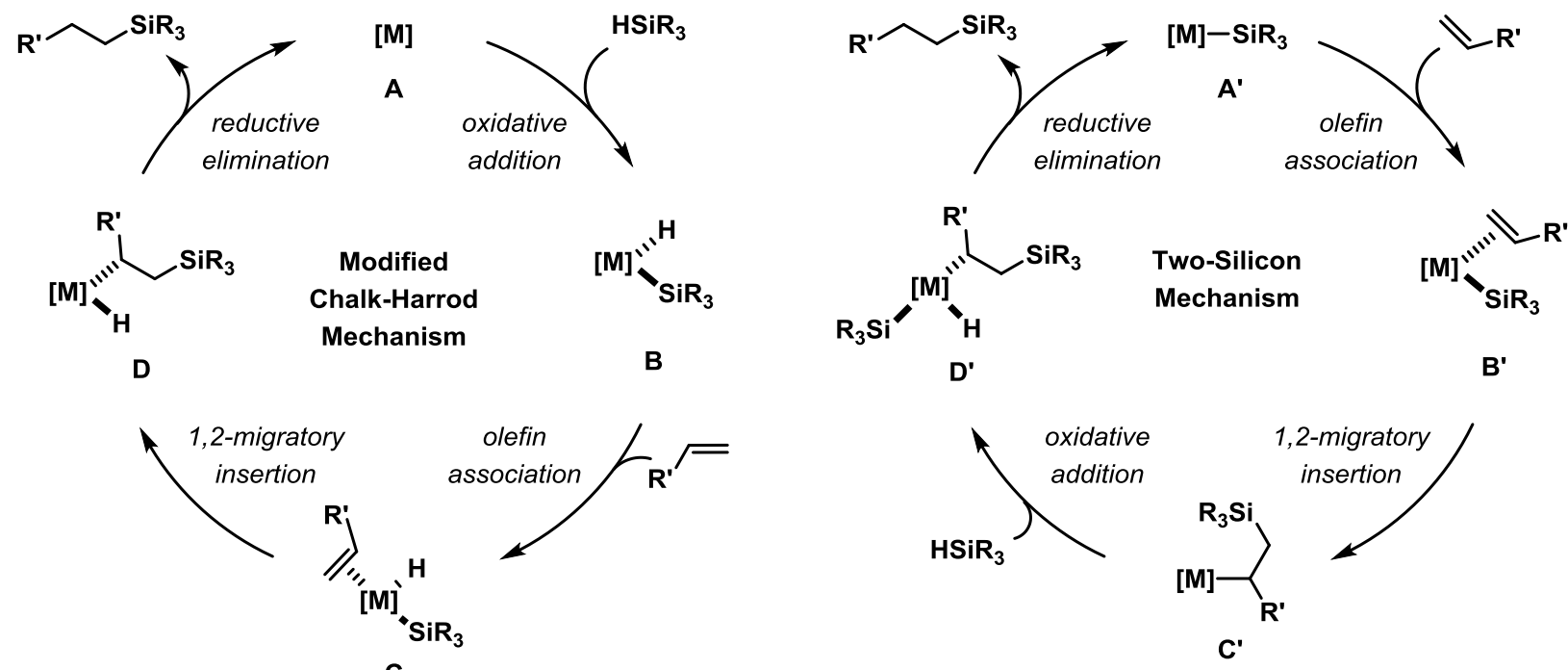

Scheme 11. Modified Chalk-Harrod Mechanisms for non-Pt catalysts ${ }^{137}$

In 1986, light scattering, TEM, and ESCA data gathered by Lewis et al. in 1986 suggested that molecular precatalysts such as Speier's and Karstedt's catalysts generate metal colloids in the presence of hydrosilanes, and that these colloids act as heterogeneous catalysts for hydrosilylation. ${ }^{144}$ Hydrosilylation was inhibited by the addition of metallic mercury, which is a well-accepted test for heterogeneous contributions to catalysis. ${ }^{145}$ Colloidal platinum, rhodium, and other metals were active catalysts for hydrosilylation, which further supported the active colloid hypothesis. ${ }^{146}$ Additionally, metals on solid supports such as $\mathrm{Pt} / \mathrm{C}$ have long been known to catalyze hydrosilylation. ${ }^{147}$ The accumulated evidence

144 (a) Lewis, L. N.; Lewis, N. J. Am. Chem. Soc. 1986, 108, 7228. For a review of catalysis by metal colloids, see: (b) Lewis, L. N. Chem. Rev. 1993, 93, 2693.

${ }^{145}$ Whitesides, G. M.; Hackett, M.; Brainard, R. L.; Lavalleye, J. P. P. M.; Sowinski, A. F.; Izumi, A. N.; Moore, S. S.; Brown, D. W.; Staudt, E. M. Organometallics 1985, 4, 1819.

${ }^{146}$ (a) Lewis, L. N.; Uriarte, R. J. Organometallics 1990, 9, 621. (b) Lewis, L. N. J. Am. Chem. Soc. 1990, 112, 5998. (c) Lewis, L. N.; Uriarte, R. J.; Lewis, N. J. Mol. Catal. 1991, 66, 105.

147 Fiedorow, R.; Wawrzynczak, A. In Education in Advanced Chemistry; Marciniec, B., Ed.; Wydawnictwo Poznanskie: Poznan, 2006; p. 327. 
suggested that colloidal platinum could be the active catalyst in olefin hydrosilylation using Speier's and Karstedt's catalysts.

However, Lewis' later studies using Karstedt's catalyst refuted their earlier conclusions by providing evidence that metal colloids formed in hydrosilylation reactions only after the depletion of the olefin substrate. ${ }^{12,148}$ This revision was based on EXAFS data showing that the types of Pt-X bonds present in metal colloids after the reaction depended on the stoichiometry of reactants, and that Pt-Pt bonds were not observed during the reaction. Additionally, NMR kinetics experiments showed that the rate of hydrosilylation was inversely proportional to the rate of colloid formation. In combination with previous work, these data elegantly demonstrated that the most active catalyst in hydrosilylation starting from Karstedt's catalyst is a homogeneous molecular species that decomposes to form platinum colloids at the completion of the hydrosilylation reaction. ${ }^{112}$

Further support for Lewis's revised conclusions came from the studies of Osborn et al. in which electron-withdrawing olefin ligands increased both the stability and activity of Karstedt's catalyst. ${ }^{149}$ Electron-poor olefins are good ligands Karstedt's catalyst because the electron-deficient olefin is able to accept electron density through $\pi$-back-bonding, which reduces the electron density at the metal center and stabilizes the electron-rich complex. Prior to Osborn et al.'s work, electron-poor olefins were thought to inhibit hydrosilylation using $\operatorname{Pt}(0)$ catalysts, but Osborn et al. demonstrated that at higher temperatures the reaction was actually accelerated in the presence of good $\pi$-accepting ligands. The effect of the $\pi$ acceptors is attributed to stabilization of the homogeneous catalyst towards metal colloid formation, which would lead to higher TOF and TON because a larger proportion of the platinum catalyst remained active during the course of hydrosilylation. ${ }^{\text {h }}$

\footnotetext{
${ }^{148}$ Lewis, L. N.; Stein, J.; Gao, Y.; Colborn, R. E.; Hutchins, G. Platinum Met. Rev. 1997, 41, 66.

149 (a) Steffanut, P.; Osborn, J. A.; Decian, A.; Fisher, J. Chem. - Eur. J. 1998, 4, 2008. (b) Hopf, A.; Dotz, K. H. J. Mol. Catal. A: Chem. 2000, 164, 191. (c) Sprengers, J. W.; Agerbeek, M. J.; Elsevier, C. J.; Kooijman, H.; Spek, A. L. Organometallics 2004, 23, 3117.
} 
Early transition metals often exist in high oxidation states with a $d^{0}$ electron count and cannot, therefore, undergo oxidative addition. Without facile two-electron oxidative addition and reductive elimination processes early transition metals, instead, catalyze hydrosilylation by yet other mechanisms (Scheme 12). ${ }^{1}$ Mechanisms based on $\sigma$-bond metathesis have been proposed, but comparatively little work has been devoted to the elucidation of mechanism using early transition metal catalysts. ${ }^{150}$ Computational $^{151}$ and some experimental ${ }^{88 b, 152}$ results suggest that more complicated mechanisms may operate for specific catalysts that involve new elementary steps to explain the interaction of a hydrosilane with a metal-alkene complex.

${ }^{150}$ Gountchev, T. I.; Tilley, T. D. Organometallics 1999, 18, 5661.

${ }^{151}$ Sakaki, S.; Takayama, T.; Sumimoto, M.; Sugimoto, M. J. Am. Chem. Soc. 2004, 126, 3332.

152 (a) Takahashi, T.; Hasegawa, M.; Suzuki, N.; Saburi, M.; Rousset, C. J.; Fanwick, P. E.; Negishi, E. J. Am. Chem. Soc. 1991, 113, 8564. (b) Corey, J. Y.; Zhu, X. H. Organometallics 1992, 11, 672. (c) Ura, Y.; Hara, R.; Takahashi, T. Chem. Lett. 1998, 195. 

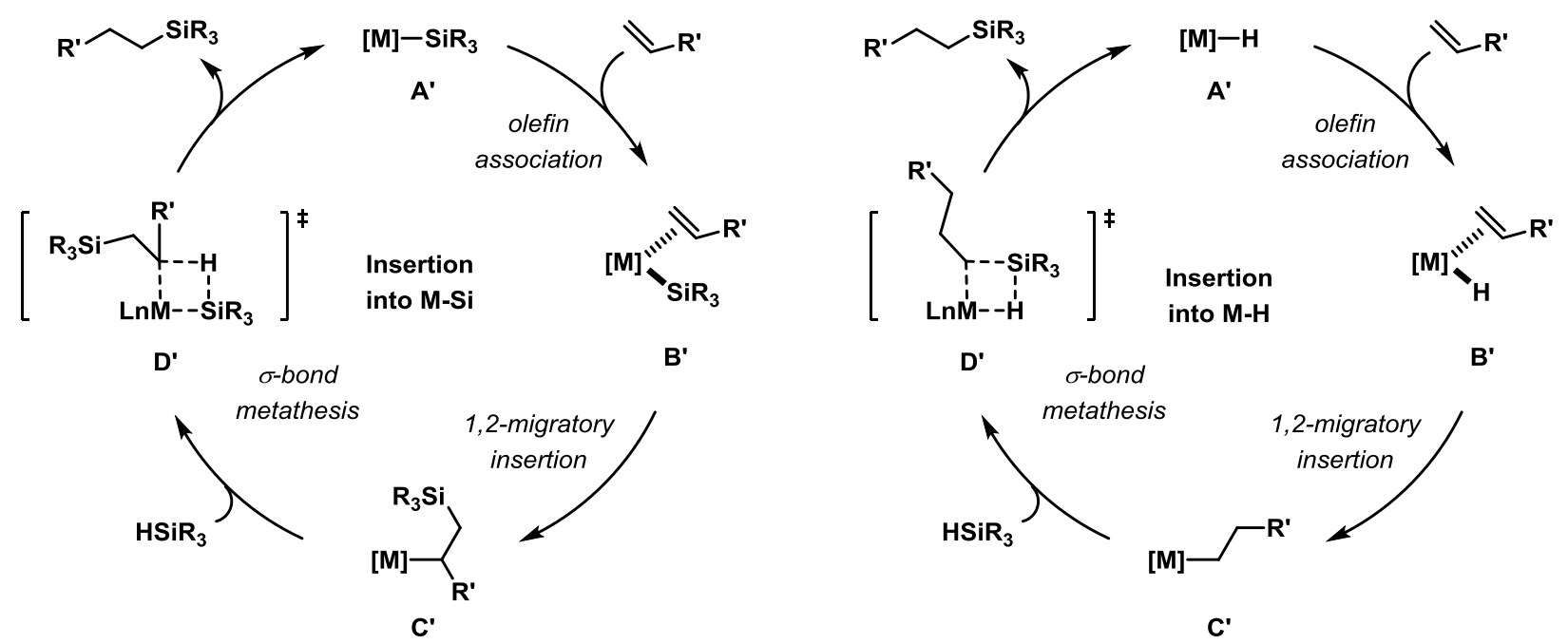

Scheme 12. Mechanism of Hydrosilylation by Early Transition Metals

\subsection{Transition Metal-Catalyzed Hydrosilylation of Conjugated Dienes}

\subsubsection{Challenges of Selective Addition to Conjugated Dienes}

Conjugated dienes, such as 1,3-butadiene, are potentially desirable substrates for hydrosilylation because butenylsilanes can be used for a variety of applications (see section 1.1). However, hydrosilylation of dienes is less studied than the hydrosilylation of other unsaturated substrates. ${ }^{1 \mathrm{~d}, \mathrm{lh}}$ In hydrosilylation reactions, 1,3-dienes can produce a wider range of products than isolated olefins. Selectivity is both unpredictable and highly dependent on the catalyst, substrate, and reaction conditions. Although a number of selective additions to dienes have been reported, most catalysts generate mixtures of isomers that are difficult to separate. The most common products of diene hydrosilylation are 1,2- and 1,4-addition products, but side reactions can generate a variety of others, including diene oligomers and saturated disilanes (Scheme 13) ${ }^{\text {ld }}$ In order for dienes to reach their potential as useful substrates for hydrosilylation reactions, methods for predictable and selective hydrosilylation must be developed. This section presents an overview of reported hydrosilylation reactions of 1,3-dienes. 


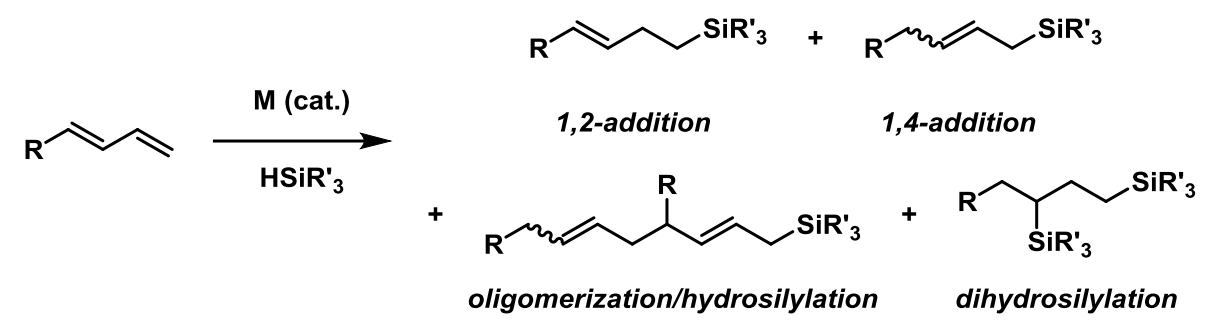

\section{Scheme 13. Common Products and Byproducts of 1,3-Diene Hydrosilylation}

Although 1,3-dienes have been studied as substrates for transition metal-catalyzed addition reactions for decades, selective transformations are relatively rare. The mechanism of diene hydrosilylation is expected to be similar to the Chalk-Harrod mechanism, but achieving selectivity is more difficult with conjugated diene substrates than with isolated olefins because a diene can interact with transition metals through $\eta^{2}$ - or $\eta^{4}$-coordination. If the diene binds to the catalyst as an $\eta^{2}$-ligand, we expect to observe similar outcomes to olefin hydrosilylation. However, if the diene binds as an $\eta^{4}$-ligand, new reaction pathways become available. In addition, relative rates of steps in the catalytic cycle can differ in reactions of dienes (compared to reactions of olefins), which can alter product distributions. 


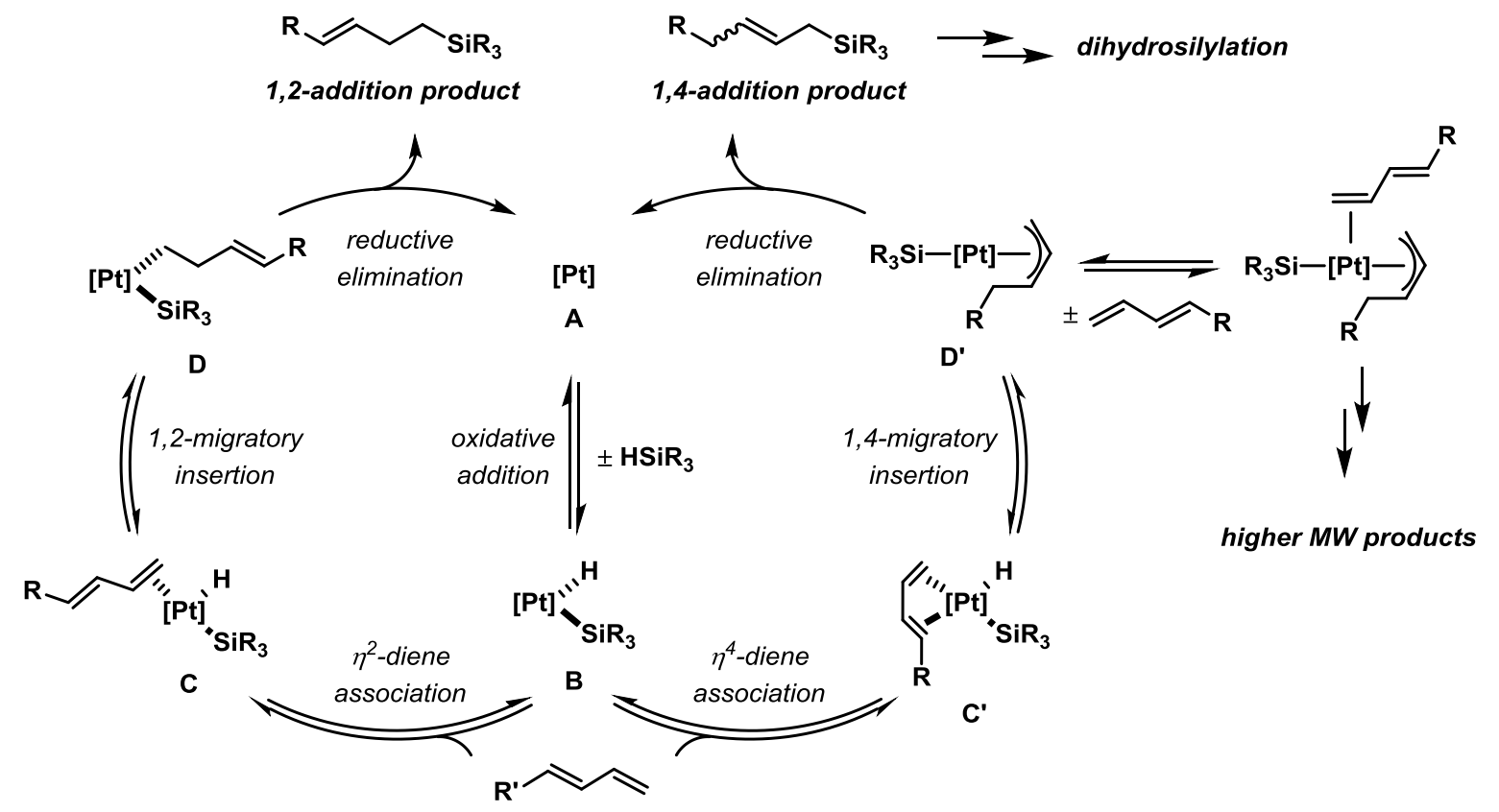

Scheme 14. Mechanism of 1,3-Diene Hydrosilylation at Platinum Catalysts

Selectivity for 1,2- or 1,4-addition may rely on the selectivity of the migratory insertion step in the Chalk-Harrod mechanism (Scheme 8). In migratory insertion, an isolated olefin can undergo 1,2- or 2,1-insertion and forms a $\sigma$-bound alkyl ligand from either of the available insertion modes. A conjugated diene can form the same $\sigma$-bound alkyl complexes, but can also form a stable $\pi$-allyl complex upon 2,1 or 1,4-insertion (Scheme 14). Because metal complexes of $\pi$-allyl ligands are often thermodynamically preferred over their $\sigma$-alkyl isomers, it is unsurprising that selective 1,4-additions to dienes are the most frequently reported. As will be discussed in Chapter 2 (see section 2.1), 1,2-addition may result from steric hindrance or other factors that prevent the formation of $\pi$-allyl intermediates.

Other side-reactions observed in the hydrosilylation of 1,3-dienes, such as oligomerization, may result from a change in the relative rates of the steps on the Chalk-Harrod cycle. Dienes have lowerenergy LUMOs than those of isolated olefins and, therefore, accept electron density through back donation $\left(d_{\mathrm{M}} \rightarrow \pi^{*}\right)$ more readily. The diene can also bind $\eta^{4}$ - as a bidentate ligand. ${ }^{153}$ Both characteristics

${ }^{153}$ Crabtree, R. H. In The Organometallic Chemistry of the Transition Metals; Wiley: Hoboken, 2005; Ch. 5. 
allow dienes (compared to olefins) to form stronger bonds with electron-rich transition metals. The rate of diene dissociation is likely to decrease upon strengthening of the metal-diene bond, which increases the concentration of the diene-bound intermediates that are implicated in side reactions such as diene dimerization and the formation of 2:1 adducts of olefin:diene (Scheme 14).

Formation of dihydrosilylated products results from competitive hydrosilylation of the reaction products. Many diene hydrosilylation catalysts are also active catalysts for the hydrosilylation of olefins. Because the product of diene hydrosilylation contains a residual carbon-carbon double bond, active catalysts may hydrosilylate the products competitively with the diene substrate. In the Chalk-Harrod mechanism, reductive elimination is likely to be the turnover-limiting step. If binding of the unsaturated substrate and migratory insertion are rapid and reversible then the product ratio would be determined by the rate of reductive elimination, which may be similar for the hydrosilylation of olefins and dienes.

As described in this section, conjugated dienes present a series of unique challenges in the development of selective addition reactions. Although a number of selective additions to dienes have been reported, the mechanism of addition and the mechanism by which selectivity is generated are not understood. The products of 1,3-diene hydrosilylation are versatile organosilanes with potential for use in many applications; however, the utilization of these products in applications demands selective and efficient synthetic methods.

\subsubsection{Hydrosilylation of Butadiene}

The first hydrosilylation reactions of butadiene were mediated by peroxides, UV-irradiation, or supported metal catalysts. ${ }^{154}$ Homogeneous metal-catalyzed hydrosilylation of butadiene was reported soon after olefin hydrosilylation, but most catalysts reported in early work are unselective and generate complex mixtures of hydrosilylation products as well as 1:2 and 2:1 adducts of diene:silane. In the

${ }^{154}$ For a comprehensive review of early hydrosilylation reactions, including hydrosilylation of dienes, see: Lukevics, E.; Belyakova, Z. V.; Pomerantseva, M. G.; Voronkov, M. G. J. Organomet. Chem. Libr. 1977, 5,1 . 
decades since these early reports, many catalyst have been reported for the hydrosilylation of conjugated dienes that generate either the 1,4-cis- or 1,4-trans-hydrosilylation products, but to our knowledge, no reported catalyst can hydrosilylate butadiene in a 1,2-selective fashion. In Chapter 2, we present the first 1,2-selective hydrosilylation of butadiene.

Early examples of butadiene hydrosilylation reactions were catalyzed by olefin hydrosilylation catalysts such as Speier's catalyst and were not optimized for yield or selectivity. Hexachloroplatinic acid catalyzes the addition of dimethylchlorosilane to butadiene to give mixtures of mono- and dihydrosilylation products (Eq. 10). Of 1:1 diene:silane adducts, the 1,4-addition product was generated in preference to the 1,2 -addition product. ${ }^{2 f, 155}$

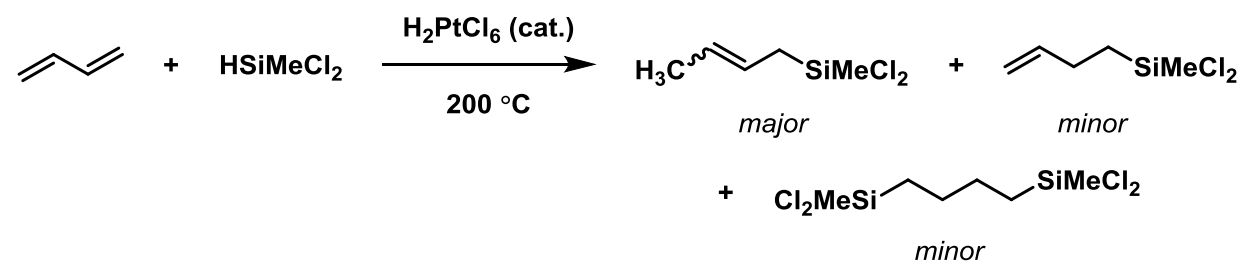

(Eq. 10)

The first palladium catalyzed hydrosilylation of butadiene was reported in 1969 . Triphenylphosphine complexes of palladium generated a 2:1 adduct of butadiene:trimethylsilane in nearly quantitative yield (Eq. 11). ${ }^{2 \mathrm{c}, 2 \mathrm{e}, 156}$ With trichlorosilane and a mixture of metallic palladium and triphenylphosphine as catalyst, a 1:1 adduct with selectivity for 1,4-addition was obtained as a mixture of cis- and trans-isomers (Eq. 12). ${ }^{2 \mathrm{~d}, 2 \mathrm{~h}}$ In both cases, the active catalytic species was proposed to be $\left(\mathrm{Ph}_{3} \mathrm{P}\right)_{2} \mathrm{Pd}(0)$, formed in situ from the added precatalysts.

${ }^{155}$ Bailey, D. L.; Pines, A. N. Ind. Eng. Chem. 1954, 46, 2363.

${ }^{156}$ Tsuji, J. Acc. Chem. Res. 1973, 6, 8. 


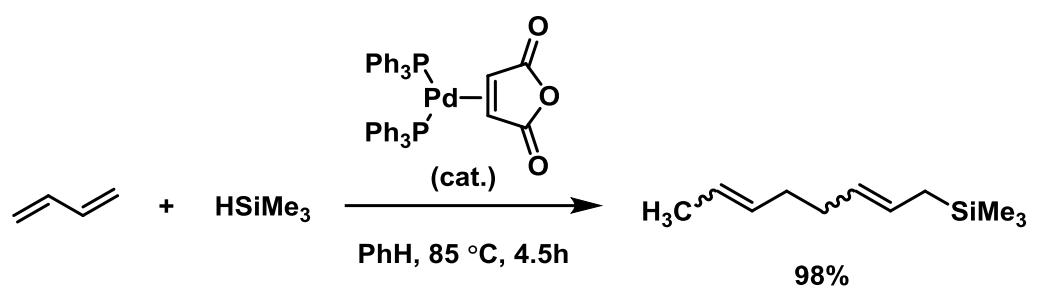

(Eq. 11)

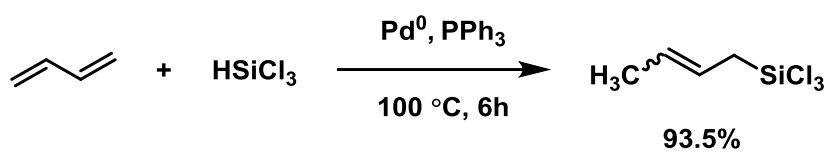

(Eq. 12)

Palladium catalysts are known to carry out the linear dimerization and oligomerization of butadiene, which suggests that the association and migratory insertion of a dienes is facile at palladium(II) catalysts. ${ }^{156,157}$ If catalysis proceeds by the Chalk-Harrod mechanism, we would expect selectivity for the formation of 1:1 vs. $2: 1$ adducts to be determined by the relative rates of $\mathrm{C}-\mathrm{Si}$ reductive elimination $(1: 1$ adducts) to the association and migratory insertion of a second molecule of diene (2:1 adducts). We expect reductive elimination to proceed more quickly at a more electron-poor metal complex. Consistent with this prediction, the formation of $1: 1$ adducts is observed in reactions of a more electron-withdrawing silane (trichlorosilane) and the formation of 2:1 adducts is observed in reactions of an electron-rich silane (trimethylsilane). These early examples demonstrate that selectivity is not only challenging in the hydrosilylation of 1,3-dienes, but also depends on a number of variables including the catalyst, substrate, silane, and reaction conditions.

Although early palladium catalysts were rarely selective for cis- or trans-products, notable exceptions were reported by Capka and Hetflejs, as well as Vaisarova et al. in 1974. Hydrosilylation

${ }^{157}$ For examples of butadiene dimerization by Pd catalysts, see: (a) Takahashi, S.; Shibano, T.; Hagihara, N. Bull. Chem. Soc. Jap. 1968, 41, 454. (b) Takahashi, S.; Yamazaki, H.; Hagihara, N. Bull. Chem. Soc. Jap. 1968, 41, 254. (c) Takahashi, S.; Yamazaki, H.; Hagihara, N. Mem. Inst. Sci. Ind. Res., Osaka Univ. 1968, 25, 125. (d) Beger, J.; Reichel, H. J. Prakt. Chem. 1973, 315, 1067. 
catalyzed by supported or polymeric palladium catalysts generated exclusively cis-1-silyl-2-butenes. ${ }^{158}$ However, although the selectivity observed in these reactions is high, the catalysts were not stable enough to be recycled after the reaction was complete (Eq. 13).

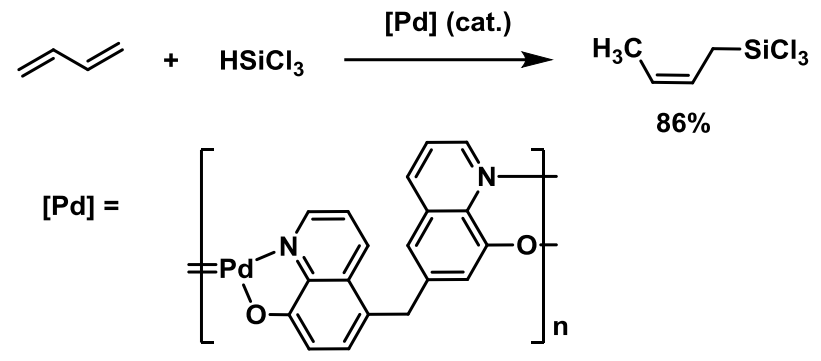

(Eq. 13)

Early examples of nickel-catalyzed diene hydrosilylation employed $\mathrm{Ni}(0)$ complexes bearing carbonyl, olefin, and/or phosphine ligands. $\mathrm{Ni}(0)$ catalysts for hydrosilylation generally favored 1,4addition, though side products from oligomerization or diene dimerization were also observed. In general, selectivity is lower than in many palladium-catalyzed reactions. Using $\mathrm{Ni}(0)$ catalysts, trimethylsilane can be added to butadiene to form a mixture of allylsilane products, 2:1 diene:silane adducts, and cyclooctadiene (Eq. 14). ${ }^{2 e}$ Because $\mathrm{Ni}(0)$ catalysts are also known to dimerize and oligomerize 1,3dienes ${ }^{159}$ production of cyclooctadiene in this example shows that the rate of dimerization is competitive with that of hydrosilylation at nickel catalysts.

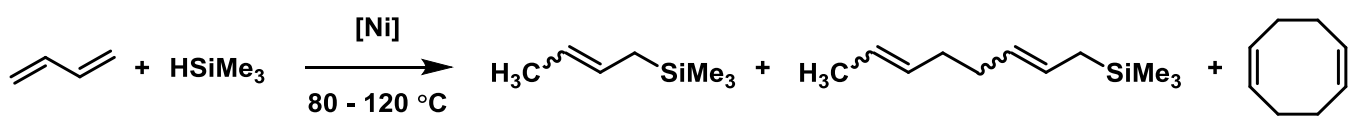

When the $\mathrm{Ni}(0)$ catalyst is prepared from a $\mathrm{Ni}(\mathrm{II})$ precursor and a reductant such as a trialkylaluminum or aluminum hydride reagent, significantly higher activity and selectivity are observed

158 (a) Capka, M.; Hetflejs, J. Collect. Czech. Chem. Commun. 1974, 39, 154. (b) Vaisarova, V.; Hetflejs, J.; Krause, H. W.; Pracejus, H. Z. Chem. 1974, 14, 105.

159 For the development of Ni-catalyzed dimerization and oligomerization of 1,3-dienes, see: (a) Bogdanovic, B.; Heimbach, P.; Kroener, M.; Wilke, G.; Hoffmann, E. G.; Brandt, J. Justus Liebigs Ann. Chem. 1969, 727, 143. (b) Brenner, W.; Heimbach, P.; Hey, H.; Mueller, E. W.; Wilke, G. Justus Liebigs Ann. Chem. 1969, 727, 161. (c) Wilke, G. Angew. Chem. Int. Ed. Engl. 1988, 27, 185. 
in the hydrosilylation of butadiene with trimethylsilane. Depending on the stoichiometry employed in the reaction, up to $95 \%$ yield of cis-but-2-enylsilanes (Eq. 15) or $45 \%$ cis,cis-octa-2,6-dienylsilanes were achieved (Eq. 16). ${ }^{160}$ Similar to the trend observed in palladium-catalyzed diene hydrosilylation, the use of electron-poor chlorosilanes increased the ratio of hydrosilylation products to side products in nickelcatalyzed reactions.
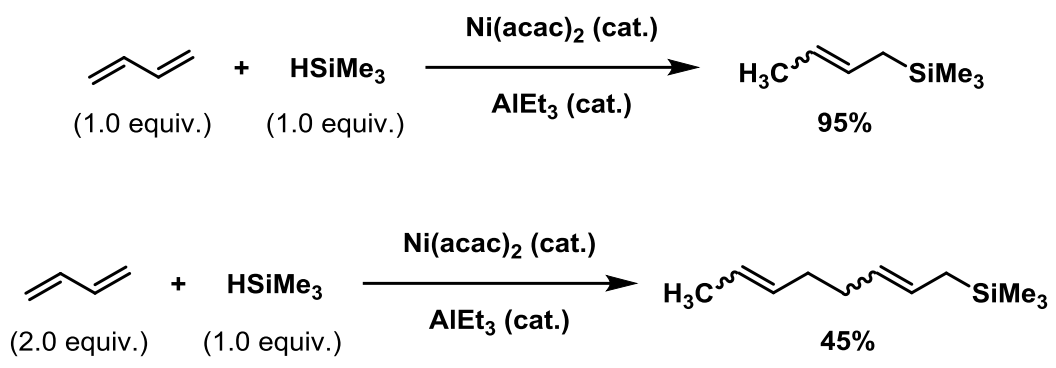

Early rhodium-catalyzed hydrosilylation of butadiene also produced mixtures that depended on the electronic properties of the silane. With trimethylsilane, $\mathrm{RhCl}\left(\mathrm{PPh}_{3}\right)_{3}$ (Wilkinson's catalyst) produced a mixture of 1,2- and 1,4-addition products along with bissilylbut-2-ene (Eq. 17). ${ }^{2 \mathrm{e}, 161}$ The bissilylbut-2ene could result from a combination of hydrosilylation and dehydrogenative silylation. Triethoxysilane, however, generated a mixture of but-2-enylsilanes as the sole products (Eq. 18). ${ }^{161}$

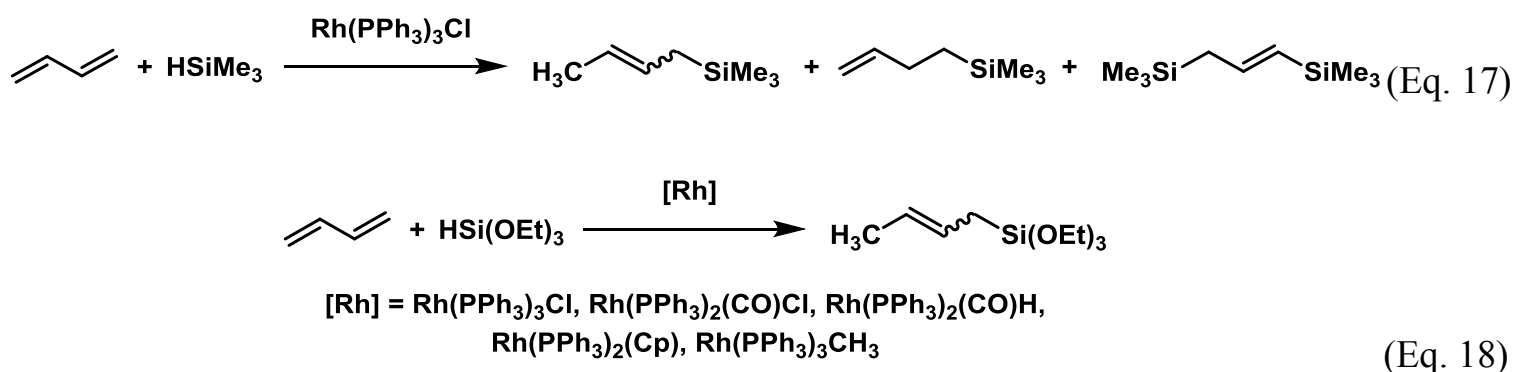

${ }^{160}$ (a) Yurev, V. P.; Salimgareeva, I. M.; Tolstikov, G. A.; Zhebarov, O. Z. Zh. Obshch. Khim. 1975, 45, 955. (b) Capka, M.; Hetflejs, J. Collect. Czech. Chem. Commun. 1975, 40, 3186. (c) Yur'ev, V. P.; Salimgareeva, I. M.; Zhebarov, O. Z.; Tolstikov, G. A. Izv. Akad. Nauk SSSR, Ser. Khim. 1975, 1888. (d) Cornish, A. J.; Lappert, M. F.; Nile, T. A. J. Organomet. Chem. 1977, 132, 133.

${ }^{161}$ Rejhon, J.; Hetflejs, J. Collect. Czech. Chem. Commun. 1975, 40, 3190. 
Cobalt catalysts such as $\mathrm{Co}_{2}(\mathrm{CO})_{8}{ }^{2 \mathrm{e}}$ (Eq. 19) and $\mathrm{CoX}_{2}$ activated by $\mathrm{NaAlH}_{2}\left(\mathrm{OCH}_{2} \mathrm{CH}_{2} \mathrm{OCH}_{3}\right)_{2}{ }^{162}$ produced complex mixtures with both trimethyl- and triethoxysilane (Eq. 20). ${ }^{2 e, 160 a}$

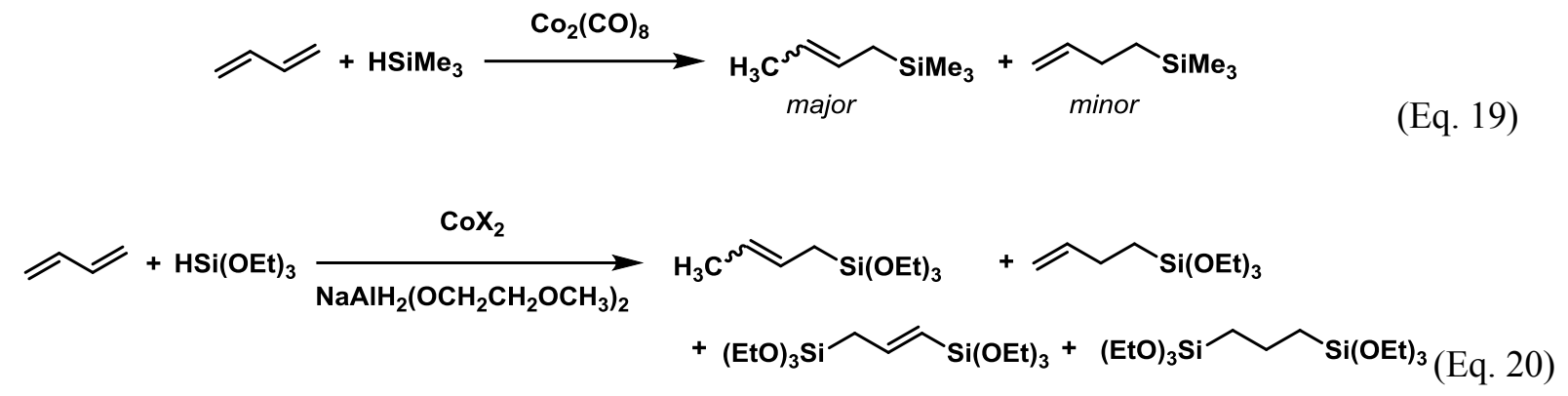

\subsubsection{Hydrosilylation of Substituted Dienes}

A wide variety of dienes are interesting substrates for hydrosilylation. However, many reported examples are limited to the few commercially available substrates including isoprene, myrcene, ocimene, 1,3-pentadiene, cyclopentadiene, 1,3-cyclohexadiene, and a handful of others. Of these, isoprene is by far the best-studied substrate for hydrosilylation. Because few functionalized dienes are commercially available, and because dienes are relatively challenging to synthesize, the substrate scope and functional group tolerance of many diene hydrosilylation reactions has not been evaluated. Instead, attention is often on the activity and/or regioselectivity of various transition metal catalysts.

Because substitution on the diene backbone creates a more hindered dienyl ligand at the metal prior to migratory insertion, substituted dienes are less likely to generate 2:1 diene:silane adducts than butadiene; however, 1:2 diene:silane adducts are still observed with some catalysts. Substitution also desymmetrizes the diene such that a wider range of constitutional isomers may be formed. Although more catalysts have been reported that generate single products in the hydrosilylation of substituted dienes than of butadiene, mixtures are still the most common result of diene hydrosilylation. Regioselective reactions predominantly form allylsilane products. Few isolated examples of 1,2-selective addition to substituted dienes have been reported and are discussed in section 1.3.5.

${ }^{162}$ Capka, M.; Hetflejs, J. Collect. Czech. Chem. Commun. 1976, 41, 1024. 
The first transition metal-catalyzed hydrosilylation of 1,3-dienes reported the addition of trichloro- and trimethylsilane to isoprene using Speier's catalyst. ${ }^{2 \mathrm{a}, 2 \mathrm{~b}, 2 \mathrm{~g}}$ The 1,4 -addition products were obtained when trichlorosilane was used (Eq. 21), but trimethylsilane produced a complex mixture (Eq. 22). Early examples using Speier's catalyst for the hydrosilylation of pentadiene ${ }^{2 g}$ (Eq. 23) also selected for 1,4-addition.
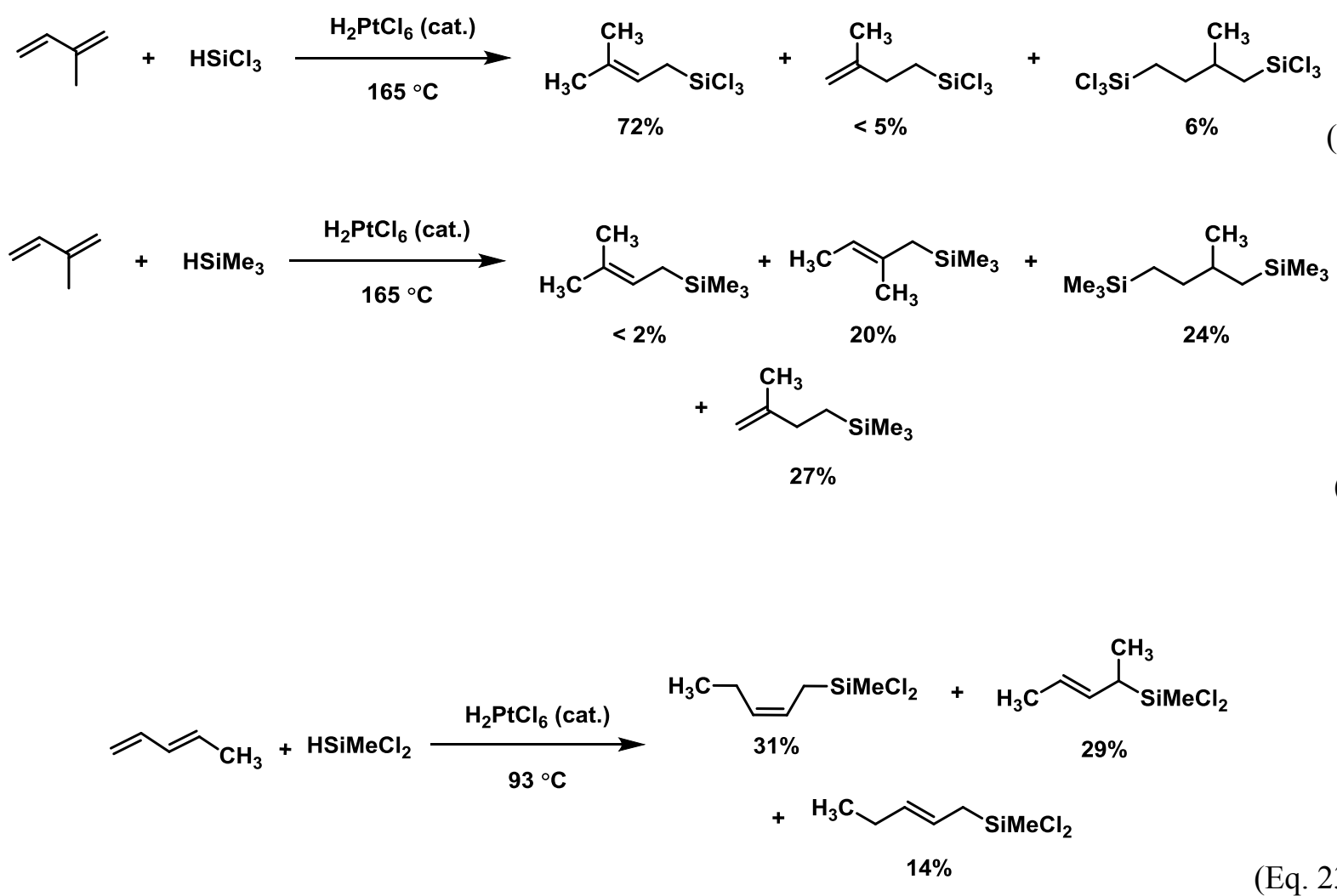

(Eq. 23)

Because of the high temperatures needed to hydrosilylate dienes with Speier's catalyst, side products from Diels-Alder addition were sometimes observed (Eq. 24). ${ }^{163}$

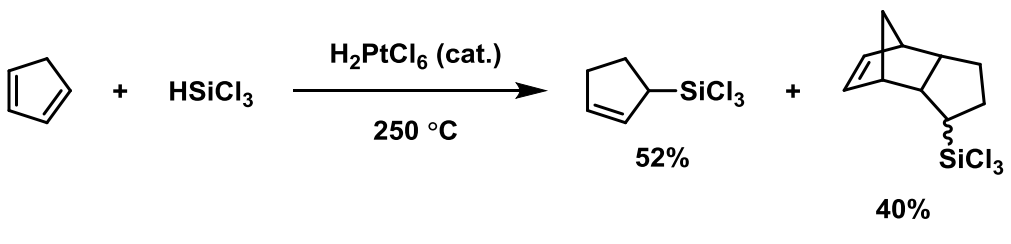

${ }^{163}$ Mironov, V. F.; Maksimova, N. G.; Nepomnina, V. V. Izv. Akad. Nauk SSSR, Ser. Khim. 1967, 329. 
Using palladium-phosphine catalysts, substituted dienes such as 1,3-pentadiene (Eq. 25), ${ }^{2 \mathrm{~h}}$ isoprene (Eq. 26), ${ }^{164}$ myrcene (Eq. 28), and ocimene ${ }^{164 b}$ (Eq. 29) gave 1:1 diene:silane adducts, with selectivity for 1,4-addition in most cases. In the case of ocimene, a mixture of products is isolated, perhaps due to the increased steric hindrance at the trisubstituted olefin of the 1,3-diene, reducing the proportion of $\eta^{4}$-bound vs. $\eta^{2}$-bound diene.
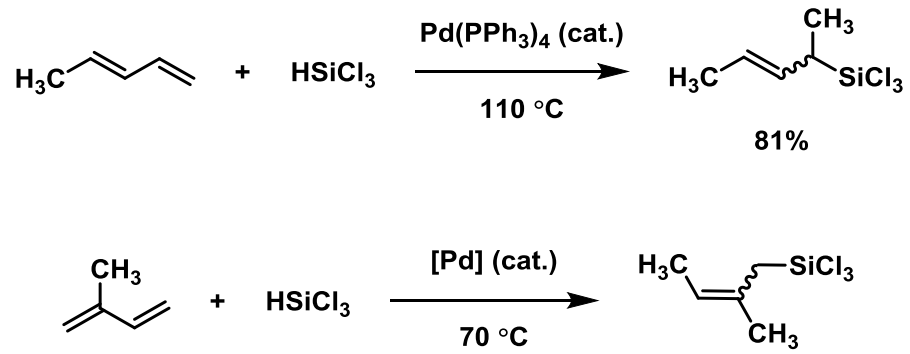

$[\mathrm{Pd}]=\mathrm{Pd}(\mathrm{PhCN})_{2} \mathrm{Cl}_{2}, \mathrm{Ph}_{3} \mathrm{P}+\mathrm{Pd}(\mathrm{PhCN})_{2} \mathrm{Cl}_{2}$
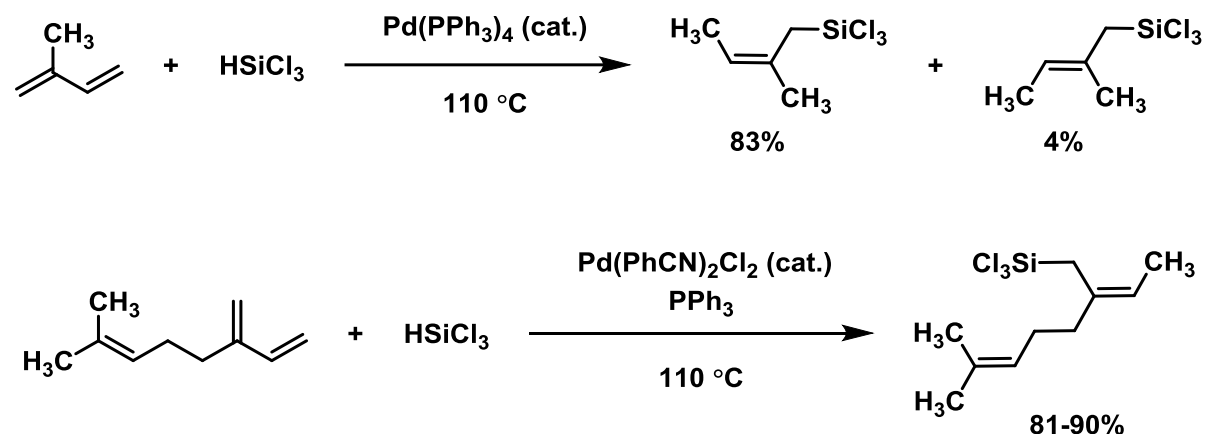

(Eq. 28)

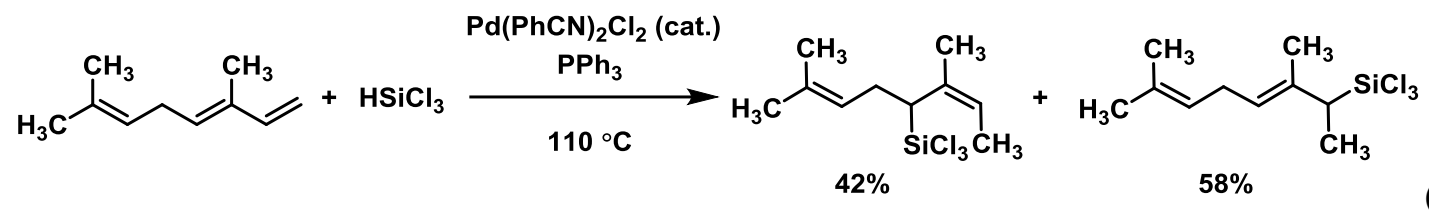

(Eq. 29)

Palladium catalysts also achieved the first asymmetric hydrosilylation of 1,3-dienes in 1972. In this case, the chiral ligand employed was either menthyldiphenylphosphine or neomenthyldiphenylphosphine and both cyclopentadiene and 1,3-cyclohexadiene were hydrosilylated using trichlorosilane to give optically

164 (a) Vaisarova, V.; Capka, M.; Hetflejs, J. Syn. Inorg. Metal-Org. Chem. 1972, 2, 289. (b) Ojima, I.; Kumagai, M. J. Organomet. Chem. 1978, 157, 359. 
active products (Eq. 30). ${ }^{165}$ Since this report, many examples asymmetric diene hydrosilylation have been reported using palladium complexes of chiral monodentate or chiral bidentate phosphine ligands. ${ }^{166}$

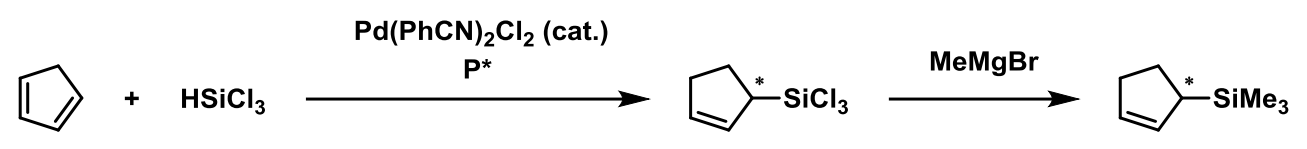

(Eq. 30)

While $\mathrm{Ni}$ (II) complexes such as $\mathrm{Cp}_{2} \mathrm{Ni}, \mathrm{NiCl}_{2}$, and $\mathrm{Ni}(\mathrm{acac})_{2}$ did not catalyze the addition of trimethylsilane to butadiene, ${ }^{2 \mathrm{~h}}$ related $\mathrm{CpNiPh}\left(\mathrm{PPh}_{3}\right)$ and $\left(\mathrm{Ph}_{3} \mathrm{P}\right)_{2} \mathrm{NiCl}_{2}$ catalyze the addition of electrophilic silanes such as trichloro- and triethoxysilanes to substituted 1,3-dienes (Eq. 31). ${ }^{167}$ Many other examples demonstrate that electron-poor silanes are necessary for hydrosilylation using $\mathrm{Ni}(0)$ or $\mathrm{Ni}(\mathrm{II})$ precatalysts, which suggests that either oxidative addition or reductive elimination should be the turnover-limiting step for nickel catalysis. ${ }^{160 a, 168}$

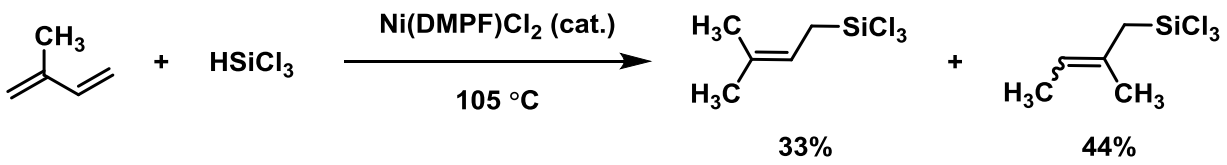

(Eq. 31)

Catalysts of rhodium and cobalt also show poor selectivity in many examples of diene hydrosilylation. When selectivity is observed using rhodium and cobalt complexes, 1,4-addition predominates and often mixtures of $c i s$ - and trans-olefins are obtained. In reactions of unsymmetrically

${ }^{165}$ Kiso, Y.; Yamamoto, K.; Tamao, K.; Kumada, M. J. Am. Chem. Soc. 1972, 94, 4373.

${ }^{166}$ For representative examples, see: (a) Hayashi, T.; Kabeta, K. Tetrahedron Lett. 1985, 26, 3023. (b) Marinetti, A.; Ricard, L. Organometallics 1994, 13, 3956. (c) Kitayama, K.; Tsuji, H.; Uozumi, Y.; Hayashi, T. Tetrahedron Lett. 1996, 37, 4169. (d) Han, J. W.; Hayashi, T. Tetrahedron: Asymmetry 2002, 13, 325. (e) Han, J. W.; Hayashi, T. Tetrahedron: Asymmetry 2010, 21, 2193.

167 Capka, M.; Hetflejs, J. Collect. Czech. Chem. Commun. 1975, 40, 3186.

168 (a) Kiso, Y.; Kumada, M.; Tamao, K.; Umeno, M. J. Organometal. Chem. 1973, 50, 297. (b) Cornish, A. J.; Lappert, M. F.; Nile, T. A. J. Organomet. Chem. 1977, 132, 133. 


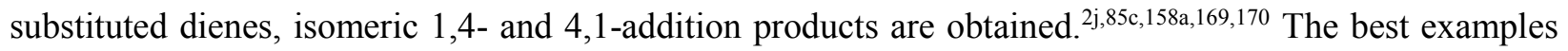
of regioselective rhodium-catalyzed hydrosilylation of isoprene are shown in Eq. 32 and Eq. $33 .{ }^{171}$
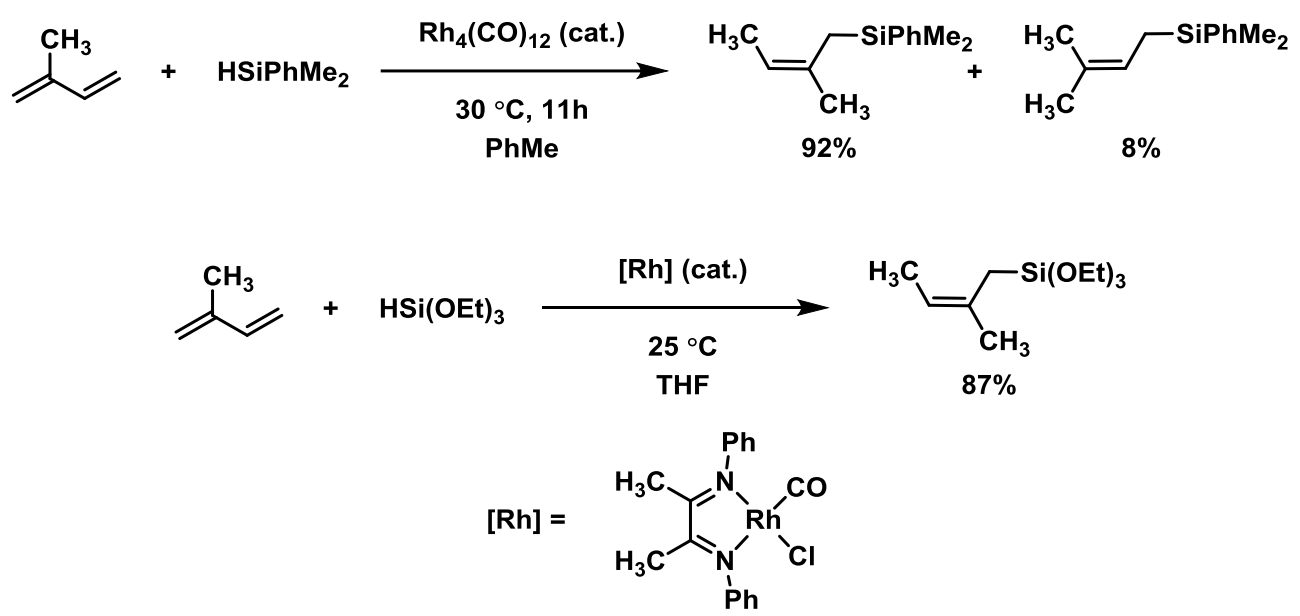

(Eq. 33)

A notable example of selective 1,4-hydrosilylation of conjugated dienes was developed recently by $\mathrm{Wu}$ et al. using a low-valent iron catalyst. The iron catalyst, supported by redox-active iminopyridine ligands, adds triethoxy-, triethyl-, and methyldiethoxysilane in a 1,4-fashion to a variety of substituted 1,3- diene substrates (Eq. 34). ${ }^{2 \mathrm{~m}}$

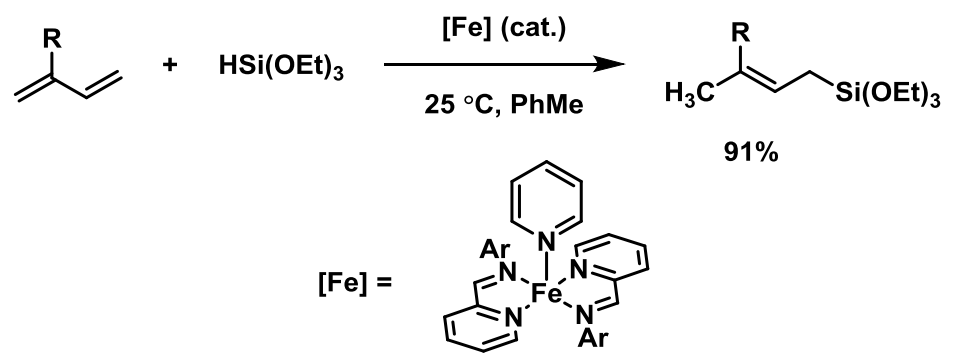

(Eq. 34)

169 For representative examples of Rh-catalyzed hydrosilylation of 1,3-dienes, see: (a) Ojima, I.; Kumagai, M. J. Organomet. Chem. 1977, 134, C6. (b) Cornish, A. J.; Lappert, M. F.; Filatovs, G. L.; Nile, T. A. J. Organomet. Chem. 1979, 172, 153. (c) Gustafsson, M.; Frejd, T. J. Chem. Soc., Perkin Trans. 1 2002, 102. (d) Gustafsson, M.; Frejd, T. J. Organomet. Chem. 2004, 689, 438.

${ }^{170}$ For a representative example of Co-catalyzed hydrosilylation of 1,3-dienes, see: Cornish, A. J.; Lappert, M. F.; Nile, T. A. J. Organomet. Chem. 1977, 136, 73.

171 (a) Brockmann, M.; Dieck, H. T.; Kleinwachter, I. J. Organomet. Chem. 1986, 309, 345,Ojima, I.; Donovan, R. J.; Clos, N. Organometallics 1991, 10, 2606. 


\subsubsection{Development of Selective Additions to Conjugated Dienes in the Ritter Research Group}

Several selective transformations of 1,3-dienes catalyzed by low-valent iron complexes have been developed in the Ritter research group, including the addition of $\alpha$-olefins, hydroboration, hydrosilylation, and polymerization. All of these transformations are selective for 1,4-addition and a defining feature is catalyst-controlled regioselectivity that can be modified by variation of the iminopyridine ligand of the catalyst. Recent work on the polymerization of isoprene led to interest in the 1,2-addition mode, which has been pursued in the work described in Chapter 2 .

Addition of $\alpha$-olefins to conjugated dienes was the first reaction of 1,3-dienes reported by the Ritter group. In this transformation, an iron (II) precatalyst was reduced in situ to form a low-valent iron catalyst in the presence of substrates (Eq. 35). Olefination of dienes proceeded with $>99: 1$ regioselectivity for olefin $\mathrm{E} / \mathrm{Z}$ configuration and selectivity for 1,4-addition was as high as $>98: 2$ for some substrates. ${ }^{172}$

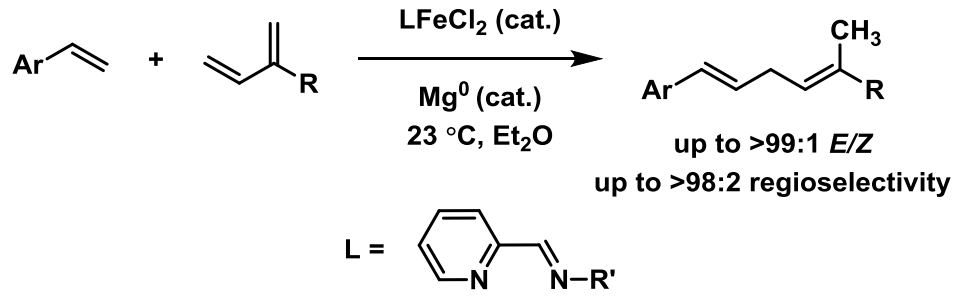

1,4-Selective hydroboration of 1,3-dienes was subsequently developed using a similar catalyst system. Variations of the substituents on the iminopyridine ligand gave either 1,4-addition (Eq. 36) or 4,1-addition (Eq. 37) with $>99: 1$ selectivity in both examples. Selectivity may be controlled by steric hindrance between substituents on the diene substrate and the ligand. Although basic functional groups arrested the reaction, ester, acetal, and ether groups were tolerated. ${ }^{173}$

\footnotetext{
${ }^{172}$ Moreau, B. T.; Wu, J. Y.; Ritter, T. Org. Lett. 2008, 11, 337.

${ }^{173}$ Wu, J. Y.; Moreau, B. T.; Ritter, T. J. Am. Chem. Soc. 2009, 131, 12915.
} 

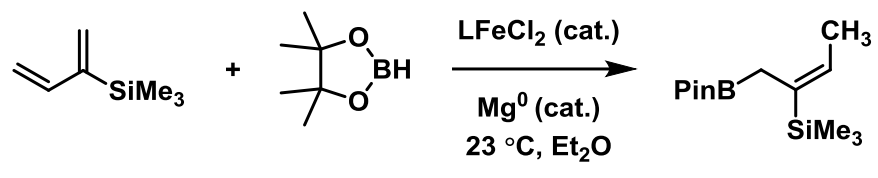

$73 \%$ yield

$>99: 14,1$-addition

$>99: 1 \mathrm{E} / \mathrm{Z}$

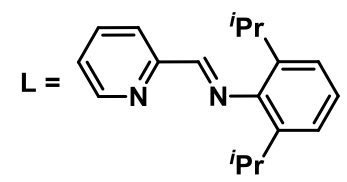

(Eq. 36)

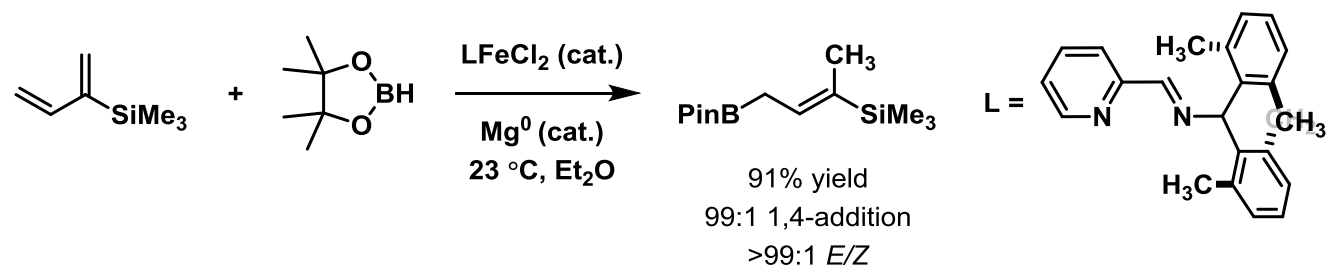

(Eq. 37)

Hydrosilylation of 1,3-dienes was developed soon after, as discussed above on page 58. In this report, $\mathrm{Wu}$ et al. demonstrate not only catalyst-controlled selectivity in the 1,4-hydrosilylation of 1,3dienes, but also utilize a well-defined low-valent iron catalyst. With this catalyst, Wu et al. were able to investigate the mechanism of 1,4 -hydrosilylation. $^{2 \mathrm{~m}}$

Most recently, a 1,4-selective polymerization of isoprene was developed by the Ritter research group using a similar iminopyridine iron catalyst. ${ }^{174}$ Selectivity for 1,4 -insertion to form either the cis(Eq. 38) or trans-olefin (Eq. 39) in the growing polymer chain was controlled by variation of the ligand on the iron catalyst. Selectivity was also observed for 3,4-insertion with some ligands (Eq. 40). Because 3,4-insertion of isoprene into a growing polymer chain represents the addition of $\mathrm{C}-\mathrm{C}$ bonds in a 1,2 selective fashion, we were inspired to investigate similar catalysts and, ultimately, new strategies for achieving 1,2-addition to conjugated dienes.
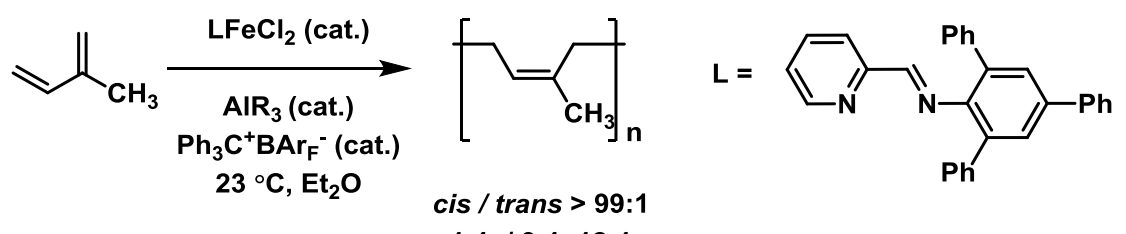

1,4- / 3,4- 12:1

${ }^{174}$ Raynaud, J.; Wu, J. Y.; Ritter, T. Angew. Chem., Int. Ed. 2012, 51, 11805. 

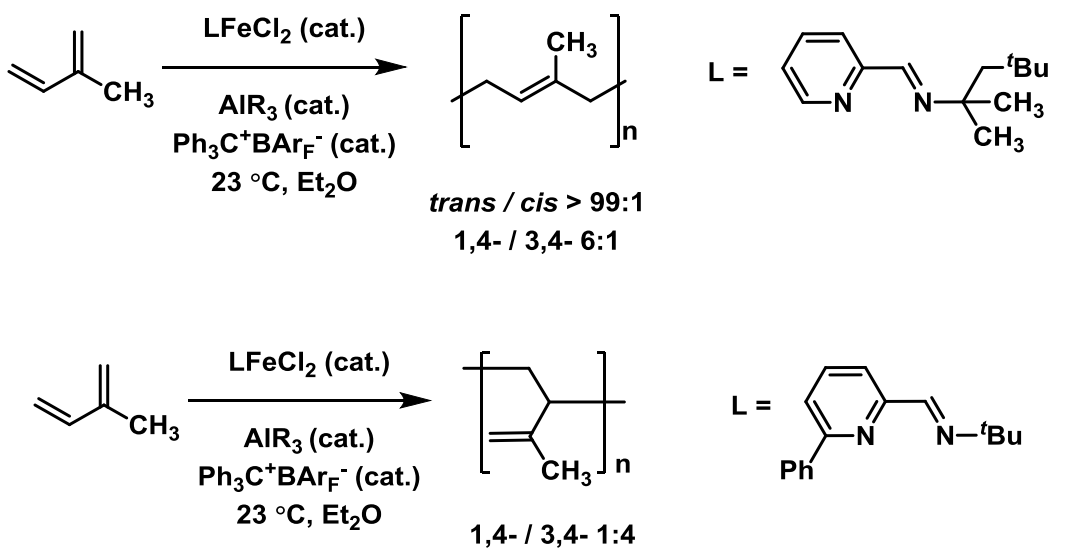

(Eq. 39)

(Eq. 40)

\subsubsection{1,2-Selective Additions to Conjugated Dienes}

Of the many transition metal-catalyzed addition reactions of 1,3-dienes, very few demonstrate selectivity for 1,2-addition. Most yield primarily 1,4-addition products, likely due to the thermodynamic favorability of $\pi$-allyl metal complexes over $\sigma$-alkyl isomers. ${ }^{153}$ Examples of 1,2 -selective addition reactions include transition metal-catalyzed hydroboration, diboration, hydrogenation, and hydrosilylation, but only three examples show generality for 1,2-selective addition; in all other reported examples, selectivity for 1,2-addition is isolated to a single substrate.

Transition metal catalysts may be more likely to generate 1,4-addition products of 1,3-dienes because $\pi$-allyl metal complexes are thermodynamically preferred over $\sigma$-alkyl complexes for many transition metals. ${ }^{153}$ Because reductive elimination is turnover limiting, intermediates $\mathbf{D}$ and D' (Scheme 14) should reach an equilibrium that reflects the relative stability of the two metal complexes. The concentration of intermediates $\mathbf{D}$ and D', which immediately precede reductive elimination, are likely to influence the product ratio unless the reaction operates under Curtin-Hammet conditions. ${ }^{175}$ Because most transition metal catalysts thermodynamically prefer a $\pi$-allyl (intermediate D') over a $\sigma$-alkyl ligand

${ }^{175}$ For seminal work on Curtin-Hammet kinetics, see: (a) Curtin, D. Y. Rec. Chem. Prog. 1954, 15, 111. For a review, see: (b) Seeman, J. I. Chem. Rev. 1983, 83, 83. 
(intermediate D), the relative proportion of D' in solution should be much greater, which can explain the selectivity for 1,4-addition at most transition metal catalysts.

1,4-Hydrosilylation predominates in addition reactions catalyzed by palladium and rhodium catalysts, while the few examples of 1,2-hydrosilylation are catalyzed by platinum complexes. The only example of 1,2-selective hydrosilylation that produces a single product utilizes $\mathrm{Pt}\left(\mathrm{PPh}_{3}\right)_{4}$ and adds $\mathrm{Cl}_{2} \mathrm{MeSiH}$ to 1,3-pentadiene to generate 3-pentenylsilane in in 86\% yield (Eq. 41). ${ }^{176}$

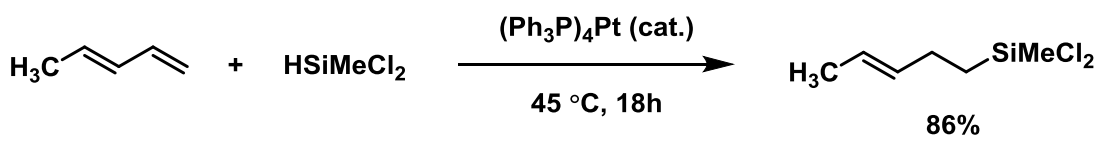

(Eq. 41)

Limited 1,2-selective addition of trichlorosilane to butadiene has been described by Rericha and Capka using the same platinum-phosphine catalyst (Eq. 42). This example is notable as higher selectivity for 1,2addition is observed than in other reported reactions of butadiene, but the low overall yield (5\%) after 5 hours at $95{ }^{\circ} \mathrm{C}$ renders this method impractical for preparation of 3-butenylsilanes.

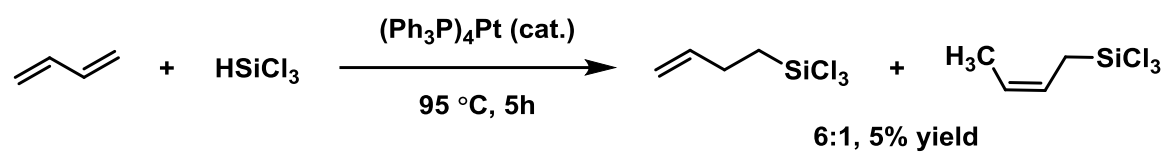

Other catalysts can also produce mixtures in which the 1,2-hydrosilylation product is the major component, but isomers are also generated in significant quantities. A similar precatalyst to that from Eq. 41 and Eq. 42, which is likely to generate the same active species in situ, forms both the 1,2-mono- and the dihydrosilylation products of isoprene, but the product of 1,4-hydrosilylation is not observed (Eq. $43) .^{2 g}$

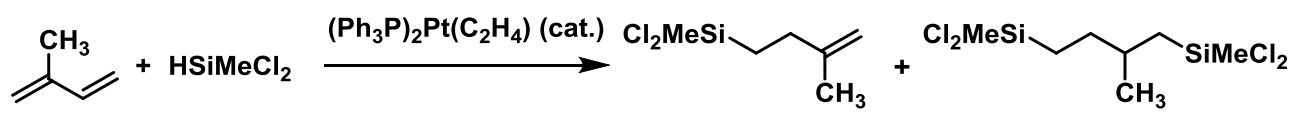

\footnotetext{
${ }^{176}$ Fink, W. Helv. Chim. Acta 1971, 54, 1304.
} 
In the case of Speier's catalyst, hydrosilylation of myrcene with trichlorosilane is partially selective for the 1,2-addition product, but also produces over-silylated products (Eq. 44$).{ }^{177}$ In contrast, other silanes and dienes show 1,4-selectivity with Speier's catalyst, indicating that regioselectivity is substratedependent.

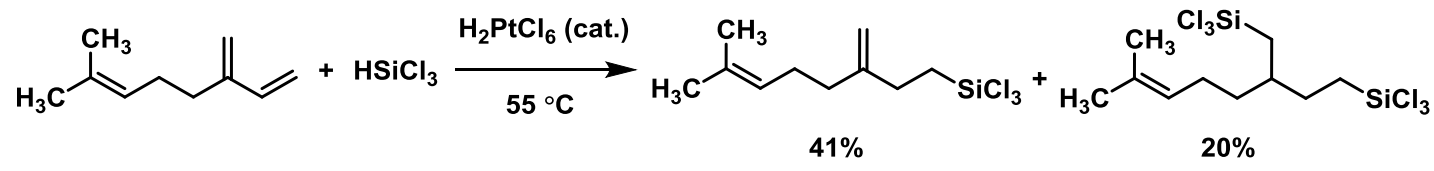

(Eq. 44)

The most general 1,2-selective addition reported to date is the diboration of conjugated dienes by an olefin-supported platinum(0) catalyst, originally reported in 1997 by Ishiyama et al. as the 1,2diboration of pentadiene. ${ }^{178}$ In this report, while $\mathrm{Pt}(\mathrm{dba})_{2}$ catalyzed the 1,2-addition of bispinacolatodiboron to pentadiene (Eq. 45), the platinum phosphine precatalyst $\mathrm{Pt}\left(\mathrm{PPh}_{3}\right)_{4}$ generated exclusively 1,4-addition products (Eq. 46).

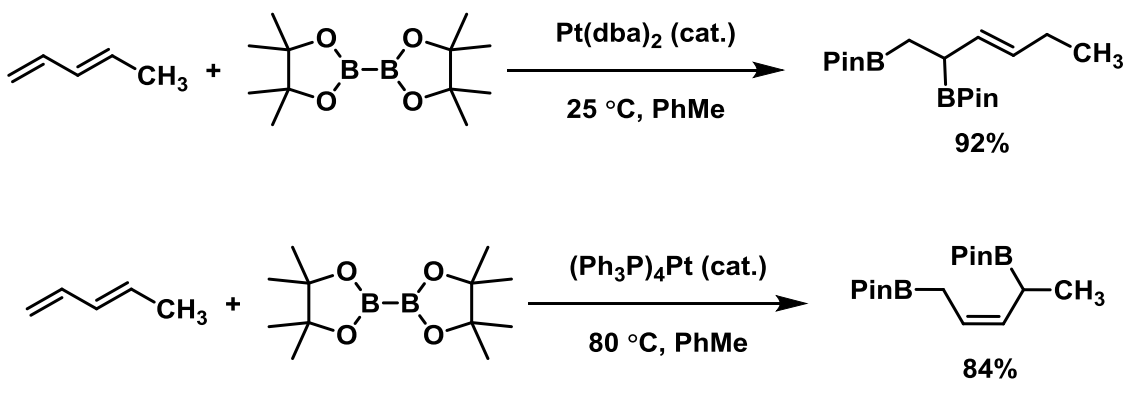

An enantioselective variation was later developed by Kliman et al. that produced enantioenriched 1,2addition products of a variety of diene substrates with pinicolatodiboron (Eq. 47), which can be further elaborated through reactions of the C-B bonds to form diols, alcohols, and other synthetically useful building blocks. ${ }^{179}$

\footnotetext{
${ }^{177}$ Nasiak, L. D.; Post, H. W. J. Organomet. Chem. 1970, 23, 91.

${ }^{178}$ Ishiyama, T.; Yamamoto, M.; Miyaura, N. Chem. Commun. 1997, 689.

${ }^{179}$ Kliman, L. T.; Mlynarski, S. N.; Ferris, G. E.; Morken, J. P. Angew. Chem. Int. Ed. Engl. 2012, 51, 521.
} 


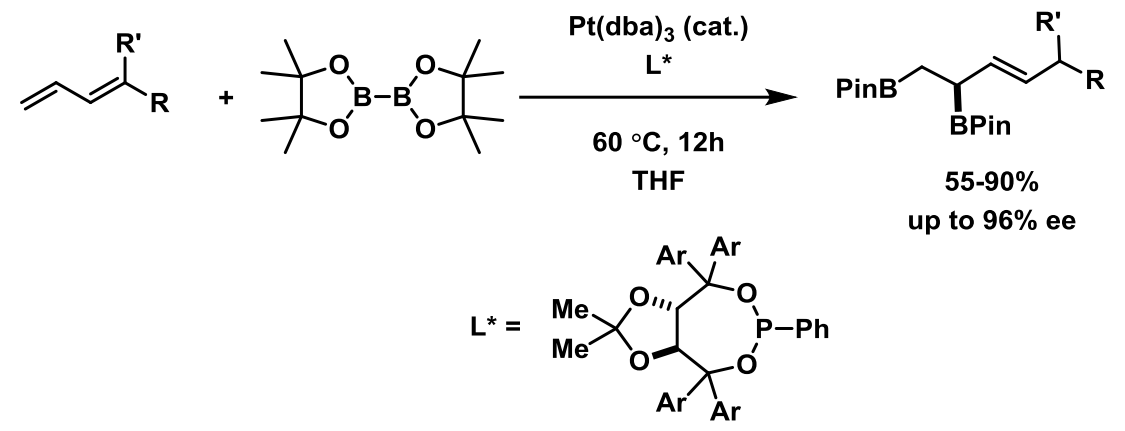

(Eq. 47)

A single example of 1,2-selective hydroboration has been reported using a nickel-phosphine catalyst and catecholborane (Eq. 48). Although no mechanistic proposal is provided, this hydroboration is also generally selective for 1,2-addition across butadiene, isoprene, myrcene, and trans-pentadiene. ${ }^{180}$

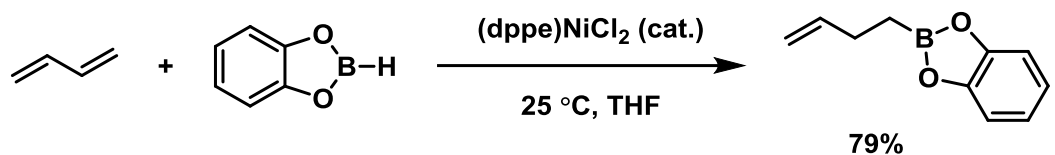

1,2-Selective partial hydrogenation of dienes has also been reported using a platinum catalyst. Bertani et al. synthesized an allylplatinum hydride precatalyst that was stable below $-20{ }^{\circ} \mathrm{C}$ (Eq. 49 ). When warmed, the allylplatinum hydride complex reductively eliminates propene, which suggests that $\mathrm{LPt}^{0}$ (diene) is the active form of the catalyst. Dienes such as butadiene, isoprene, and 2,3dimethylbutadiene were partially hydrogenated to the terminal olefin products under these conditions. Under these conditions, no butane was detected and isolation of other butene isomers was not reported. ${ }^{181}$

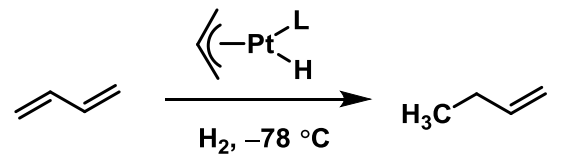

${ }^{180}$ Zaidlewicz, M.; Meller, J. Tetrahedron Lett. 1997, 38, 7279.

${ }^{181}$ Bertani, R.; Carturan, G.; Scrivanti, A. Angew. Chem. 1983, 95, 241. 


\section{RESULTS AND DISCUSSION}

The aim of this research was the development of a transition metal catalyst for the 1,2-selective hydrosilylation of 1,3-dienes, including butadiene. Butenylsilanes, such as the product of 1,2hydrosilylation of butadiene, can act as coupling reagents to covalently link the surface of silicate-based sol-gel materials with hydrophobic olefin polymers. ${ }^{1}$ Coupling reagents contain a silicon group with hydrolysable ligands and an $\alpha$-olefin from which olefin polymerization can be initiated. ${ }^{1 b}$ Although vinyltriethoxysilane and 1-hexenyltriethoxysilane are currently used as coupling reagents, vinyl silanes are less active than $\alpha$-olefins for initiating olefin polymerization ${ }^{1 \mathrm{~d}}$ and hexenylsilanes are produced from an expensive feedstock. ${ }^{2}$ If butenylsilanes could by synthesized from butadiene by transition metalcatalyzed hydrosilylation, an efficient and practical method for industrial scale synthesis, butenylsilanes could function as an inexpensive, active coupling agent for hybrid materials synthesis.

Previous work has demonstrated the capricious nature of 1,3-diene substrates in transition metalcatalyzed hydrosilylation reactions. As discussed in section 1.3.5 (page 61), specific challenges exist for achieving selective addition to 1,3-dienes at transition metal catalysts. Selective 1,4-additions to dienes can be achieved with known catalysts by utilizing the thermodynamic favorability of metal-allyl intermediates (products of 1,4- or 2,1-migratory insertion) over their $\sigma$-alkyl isomers (products of 1,2migratory insertion). ${ }^{3}$ In contrast, 1,2-selective addition to conjugated dienes is rare. General methods for

${ }^{1}$ (a) Arkles, B. CHEMTECH 1977, 7, 766. (b) Plueddemann, E. P. Silane Coupling Agents; 2 ed.; Plenum Press: New York, 1991. (c) Lewis, L. N.; Stein, J.; Gao, Y.; Colborn, R. E.; Hutchins, G. Platinum Met. Rev. 1997, 41, 66. (d) Brook, M. A. Silicon in Organic Organometallic, and Polymer Chemistry; WileyVCH Verlag, 2000. (e) Ab Rahman, I.; Padavettan, V. J. Nanomater. 2012, 132424. (g) Cui, S.; Lin, B.; Liu, Y.; Liu, X.; Shen, X.; Han, G.; Zhou, X. Curr. Nanosci. 2012, 8, 797.

2 (a) Saiki, T. Phosphorus, Sulfur Silicon Relat. Elem. 2008, 183, 1556. (b) Tanaka, H. Jpn. Kokai Tokkyo Koho J.P. 2008174523 A, 2008.

${ }^{3}$ (a) Shiihara, I.; Hoskyns, W. F.; Post, H. W. J. Org. Chem. 1961, 26, 4000. (b) Mamedaliev, Y. G.; Mamedov, M.; Sadykh-Zade, S. I.; Akhmedov, I. M.; Salimov, M. A. Azerb. Khim. Zh. 1962, 9. (c) Takahashi, S.; Shibano, T.; Hagihara, N. Chem. Commun. 1969, 161. (d) Tsuji, J.; Hara, M.; Ohno, K. Chem. Commun. 1971, 247. (e) Takahashi, S.; Shibano, T.; Kojima, H.; Hagihara, N. Organometal. 
1,2-addition are limited to three examples and the factors leading to 1,2-selectivity in these cases are not understood. ${ }^{4}$ Few isolated examples of 1,2-hydrosilylation for specific substrates or as part of product mixtures have been reported, but 1,2-selectivity is substrate-dependent and does not extend to butadiene in any reported case. ${ }^{3 \mathrm{~g}, 5,6}$ In this work, we propose that a transition metal catalyst that excludes formation of $\pi$-allyl intermediates upon migratory insertion of 1,3-dienes would result in catalyst-controlled 1,2selective addition.

This chapter describes the discovery and development of cyclometallated-phosphine complexes of $\mathrm{Pt}(\mathrm{II})$ that catalyze the 1,2-selective addition of triethoxysilane to butadiene and other 1,3-dienes. The first section describes the strategy we employed in our investigation of 1,2-selective hydrosilylation. Next we describe the discovery of platinum catalysts for 1,2-selective hydrosilylation. The last section discusses possible mechanisms for 1,2-selective hydrosilylation, describes experimental evidence for a $\mathrm{Pt}(\mathrm{II}) / \mathrm{Pt}(\mathrm{IV})$ catalytic cycle, and presents evidence against certain other mechanisms.

Chem. Syn. 1971, 1, 193. (f) Belyakova, Z. V.; Pomerantseva, M. G.; Popkov, K. K.; Efremova, L. A.; Golubtsov, S. A. Zh. Obshch. Khim. 1972, 42, 889. (g) Swisher, J. V.; Zullig, C. J. Org. Chem. 1973, 38, 3353. (h) Hayashi, T.; Kabeta, K. Tetrahedron Lett. 1985, 26, 3023. (i) Wu, J. Y.; Stanzl, B. N.; Ritter, T. J. Am. Chem. Soc. 2010, 132, 13214.

${ }^{4}$ For 1,2-selective hydroboration, see: (a) Zaidlewicz, M.; Meller, J. Tetrahedron Lett. 1997, 38, 7279. For 1,2-selective diboration see: (b) Ishiyama, T.; Yamamoto, M.; Miyaura, N. Chem. Commun. 1997, 689. (c) Kliman, L. T.; Mlynarski, S. N.; Ferris, G. E.; Morken, J. P. Angew. Chem. Int. Ed. Engl. 2012, 51,521 .

${ }^{5}$ For reviews of 1,3-diene hydrosilylation, see: (a) Ojima, I.; Kogure, T. Rev. Silicon, Germanium, Tin Lead Compd. 1981, 5, 7. (b) Ojima, I.; Patai, S., Rappoport, Z., Eds.; Wiley: Chichester, 1989; Ch. 25.

${ }^{6}$ For examples of 1,2-selective hydrosilylation of specific substrates, see: (a) Andrianov, K. A.; Bagratashvili, G. D.; Kantariya, M. L.; Sidorov, V. I.; Khananashvili, L. M.; Tsitsishvili, G. V. J. Organomet. Chem. 1965, 4, 440. (b) Nasiak, L. D.; Post, H. W. J. Organomet. Chem. 1970, 23, 91. (c) Fink, W. Helv. Chim. Acta 1971, 54, 1304. (d) Rericha, R.; Capka, M. Collect. Czech. Chem. Commun. 1974, 39, 144. 


\subsection{Strategy for 1,2-Selective Hydrosilylation Catalyzed by Transition Metals}

Our strategy for developing a selective 1,2-hydrosilylation of 1,3-dienes stems from our hypothesis that transition metal-catalyzed 1,4-addition reactions proceed via $\pi$-allyl complex intermediates (see Scheme 14 in Chapter 1, page 47). As demonstrated in Scheme 14, the 1,4-addition pathway proceeds via an allyl complex, which has been drawn as the $\pi$-bound isomer (1,4-insertion). The 1,2-addition pathway, instead, proceeds via a non-conjugated homoallyl complex that reacts like a $\sigma$-alkyl ligand (1,2-insertion). One way to block the 1,4-addition pathway would be to prevent the formation of $\pi$ bound allyl complexes.

One of the simplest differences between a $\sigma$-alkyl ligand and a $\pi$-allyl ligand is the number of coordination sites the ligand occupies at the metal center (Scheme 15). A $\sigma$-alkyl (or $\sigma$-allyl) ligand is a simple X-type $2 \mathrm{e}^{-}$donor and requires only one coordination site. A $\pi$-bound allyl ligand is an X,L-type $4 \mathrm{e}^{-}$donor and requires two coordination sites. We hypothesized that by restricting the number of available coordination sites the $\pi$-bound allyl isomer would be excluded.

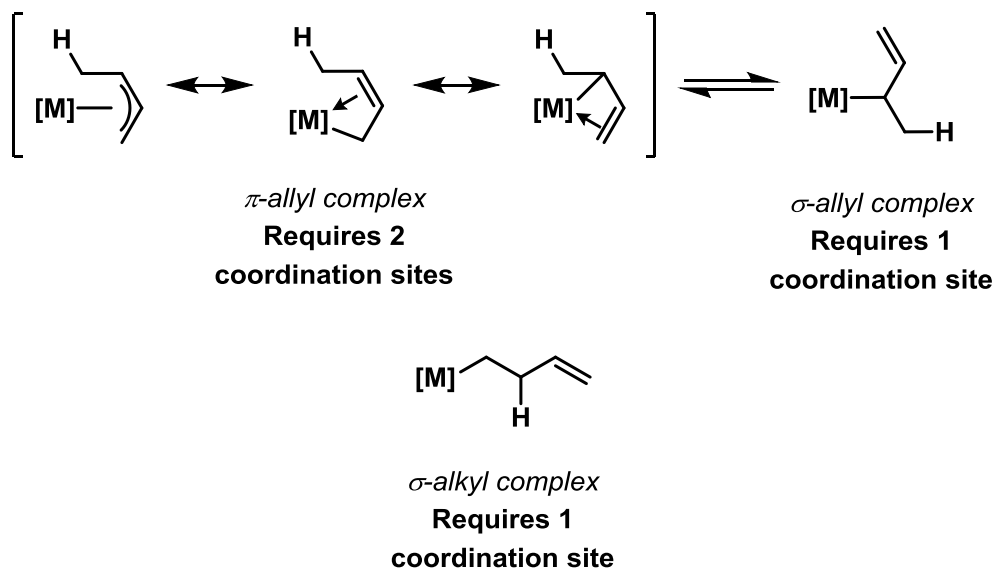

Scheme 15. Coordination of Allyl and Alkyl Ligands to Transition Metals 

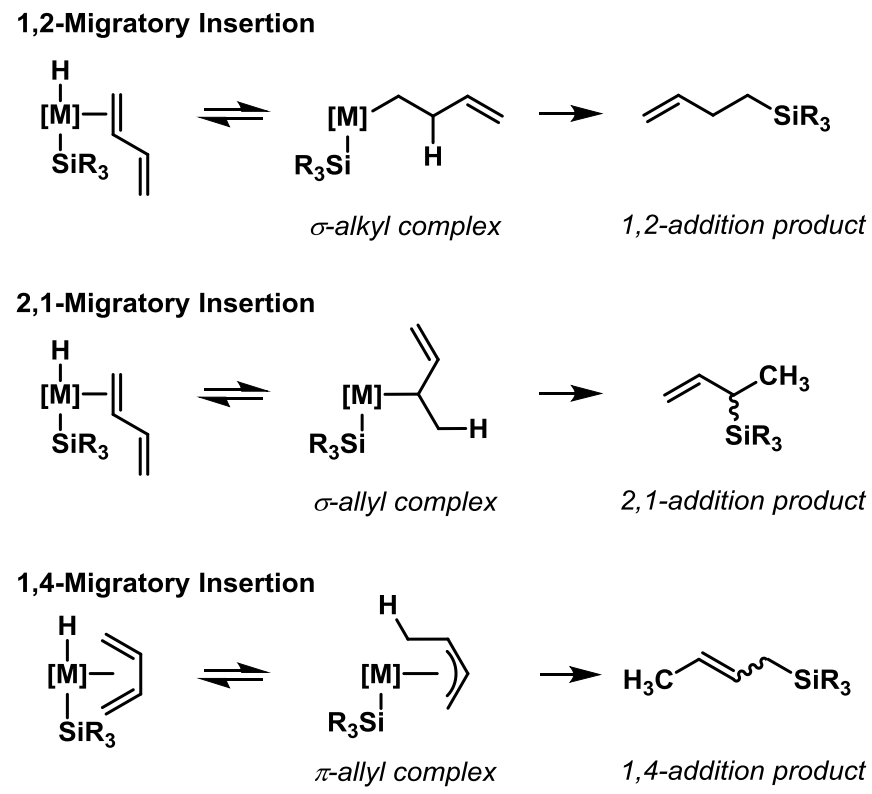

\section{Scheme 16. Migratory Insertion Modes of Conjugated Dienes Bound to Transition Metals}

A second mechanism for the formation of 1,4-addition products exists through a 2,1-migratory insertion to form a $\sigma$-allyl complex (Scheme 16). Were the formation of a $\sigma$-bound allyl complex thermodynamically favored over the formation of a $\sigma$-bound alkyl ligand, 1,4-addition would be preferred over 1,2-addition without the formation of $\pi$-allyl intermediates. $\sigma$-Bound allyl complexes experience energetic stabilization from conjugation, ${ }^{7}$ but the $\sigma$-alkyl complex proposed for 1,2-addition is bound at a terminal carbon while the proposed $\sigma$-allyl is bound at an internal carbon. Platinum-carbon bonds are destabilized by steric interactions with other groups bound to the ligated carbon. ${ }^{8}$ We hypothesized that a Group 10 transition metal catalyst would favor 1,2-insertion over 2,1-insertion as has been demonstrated in the literature for isolated olefins. ${ }^{9}$

\footnotetext{
${ }^{7}$ Fleming, I.; Wiley: Chichester, 2007; p.19-21.

${ }^{8}$ For studies of thermodynamic stability of migratory insertion products, see: (a) Arnek, R.; Zetterberg, K. Organometallics 1987, 6, 1230. (b) Reger, D. L.; Garza, D. G.; Baxter, J. C. Organometallics 1990, 9, 873. (c) Stromberg, S.; Zetterberg, K.; Siegbahn, P. E. M. J. Chem. Soc., Dalton Trans. 1997, 4147. (d) Shiotsuki, M.; White, P. S.; Brookhart, M.; Templeton, J. L. J. Am. Chem. Soc. 2007, 129, 4058.

${ }^{9}$ For computational studies of migratory insertion into Pt-H bonds, see: (a) Sakaki, S.; Mizoe, N.; Musashi, Y.; Sugimoto, M. J. Mol. Struct.: THEOCHEM 1999, 461-462, 533. (b) Sakaki, S.; Mizoe, N.;
} 
A final consideration in the design of a 1,2-selective hydrosilylation reaction is the chemoselectivity of the migratory insertion step. In order for the 1,2-addition product to be formed, migratory insertion into $\mathrm{M}-\mathrm{H}$ must be preferred over $\mathrm{M}-\mathrm{Si}$. Although the Chalk-Harrod mechanism is well-established for platinum complexes, the lighter Group 10 metals may catalyze hydrosilylation via the modified Chalk-Harrod mechanism. We proposed that by adding an oxyanion to the ligand backbone, the silicon ligand could be reversibly tethered as a pentacoordinate silicate, which would reduce the rate of migratory insertion into the $\mathrm{M}-\mathrm{Si}$ bond and encourage insertion into the $\mathrm{M}-\mathrm{H}$ bond (Scheme 17). We initially selected iminopyridine ligands because of their tunability, ease of synthesis, and the suggestion of 1,2-selectivity observed in the polymerization of isoprene at an iminopyridine iron complex.

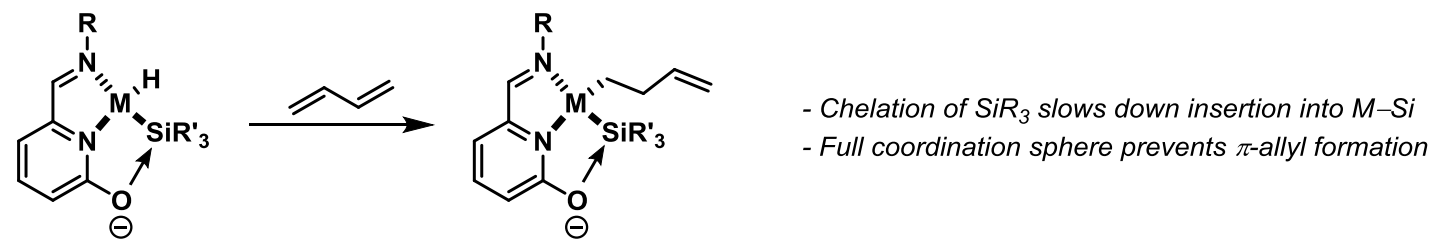

\section{Scheme 17. Strategy for the 1,2-Selective Hydrosilylation of Conjugated Dienes}

\subsection{Development of Platinum Catalysts for 1,2-Selective Hydrosilylation of Butadiene}

\subsubsection{Catalyst Discovery}

A collection of bidentate iminopyridone ligands and other bidentate ligands with pendant hydroxyl groups were synthesized and tested as ligands for Group 10 metals in the hydrosilylation of butadiene. Although many complexes of nickel were efficient 1,4-selective hydrosilylation catalysts, no 1,2-selective hydrosilylation was observed starting from a nickel precursor. Several ligands showed modest selectivity for 1,2-addition (up to 3:1 1,2-:1,4-addition) in combination with $\mathrm{Pd}(\mathrm{II})$ and $\mathrm{Pt}(\mathrm{II})$ precursors, but yields of hydrosilylated products were low $(<10 \%)$ in all cases (Figure 3). Control experiments showed that the hydroxyl group on the ligand was unnecessary for 1,2-selectivity. Despite

Sugimoto, M.; Musashi, Y. Coord. Chem. Rev. 1999, 190-192, 933. (c) Giorgi, G.; De Angelis, F.; Re, N.; Sgamellotti, A. J. Mol. Struct.: THEOCHEM 2003, 623, 277. 
the significant variations in steric demand and coordination geometry, yield and selectivity did not significantly change from one ligand to another within this set.

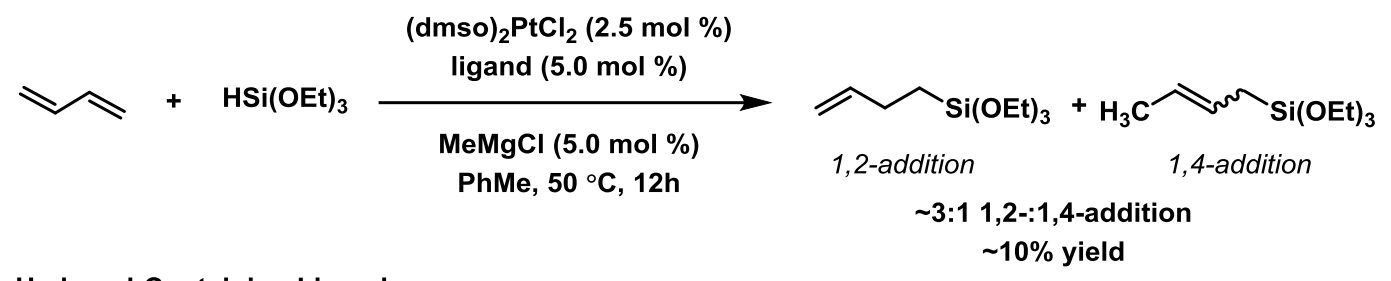

Hydroxyl-Containing Ligands:

Control Ligands:
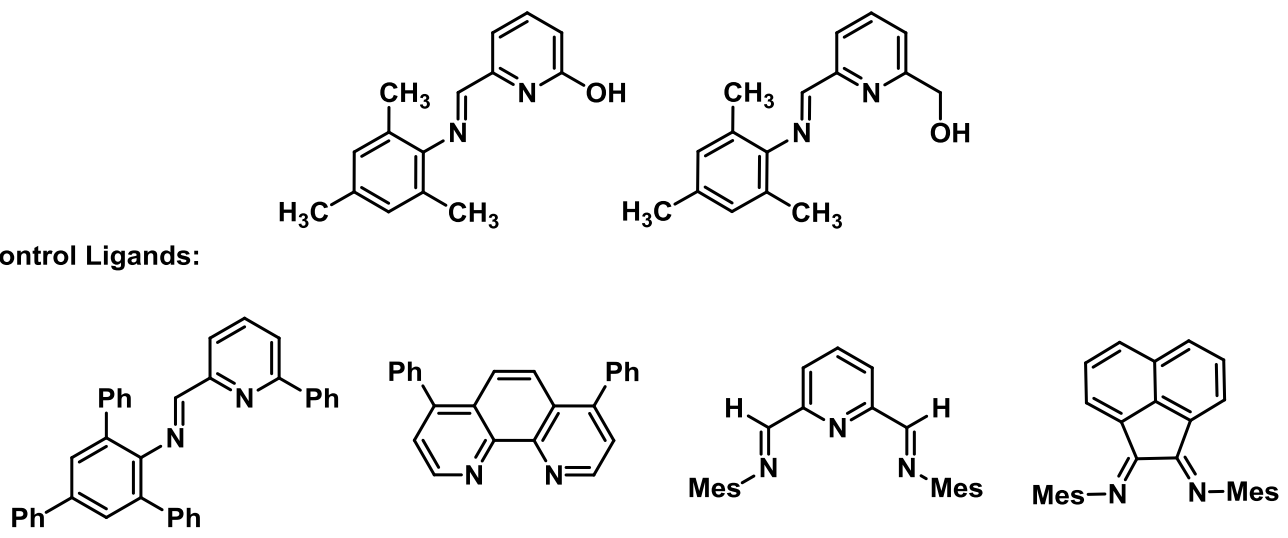

Figure 3. Early ligands for the 1,2-selective hydrosilylation of butadiene

After determining that the pendant hydroxyl group was unnecessary to achieve 1,2-selectivity, we investigated other common monodentate and bidentate ligands for platinum, including phosphine ligands and NHC ligands. In general, the strong $\sigma$-donor ligands shown in Table 3 resulted in higher yields of hydrosilylation products than the nitrogen-based ligands shown in Figure 3. Notably, ligands with similar size and electronic properties showed selectivity for different products when tested as ligands for platinum-catalyzed hydrosilylation. For example, the hydrosilylation of butadiene using tri-tertbutylphosphine was selective for 1,2-addition, but the very similar tricyclohexylphosphine was selective for 1,4-addition. The difference between the ligands that showed selectivity for 1,2-addition and the ligands that showed selectivity for 1,4-addition seemed, based on these comparisons, not to be one of electronic properties, steric demand, or ligand structure. Instead, we hypothesized that the determining 
factor may be the ready accessibility of a $\mathrm{C}-\mathrm{H}$ bond at a distance of 3-4 bonds from the $\sigma$-donating atom of the ligand, which would allow for facile cyclometallation to form a bidentate metallacycle in situ.

Table 3. Ligands Demonstrating 1,2- vs. 1,4-Selectivity in the Hydrosilylation of Butadiene
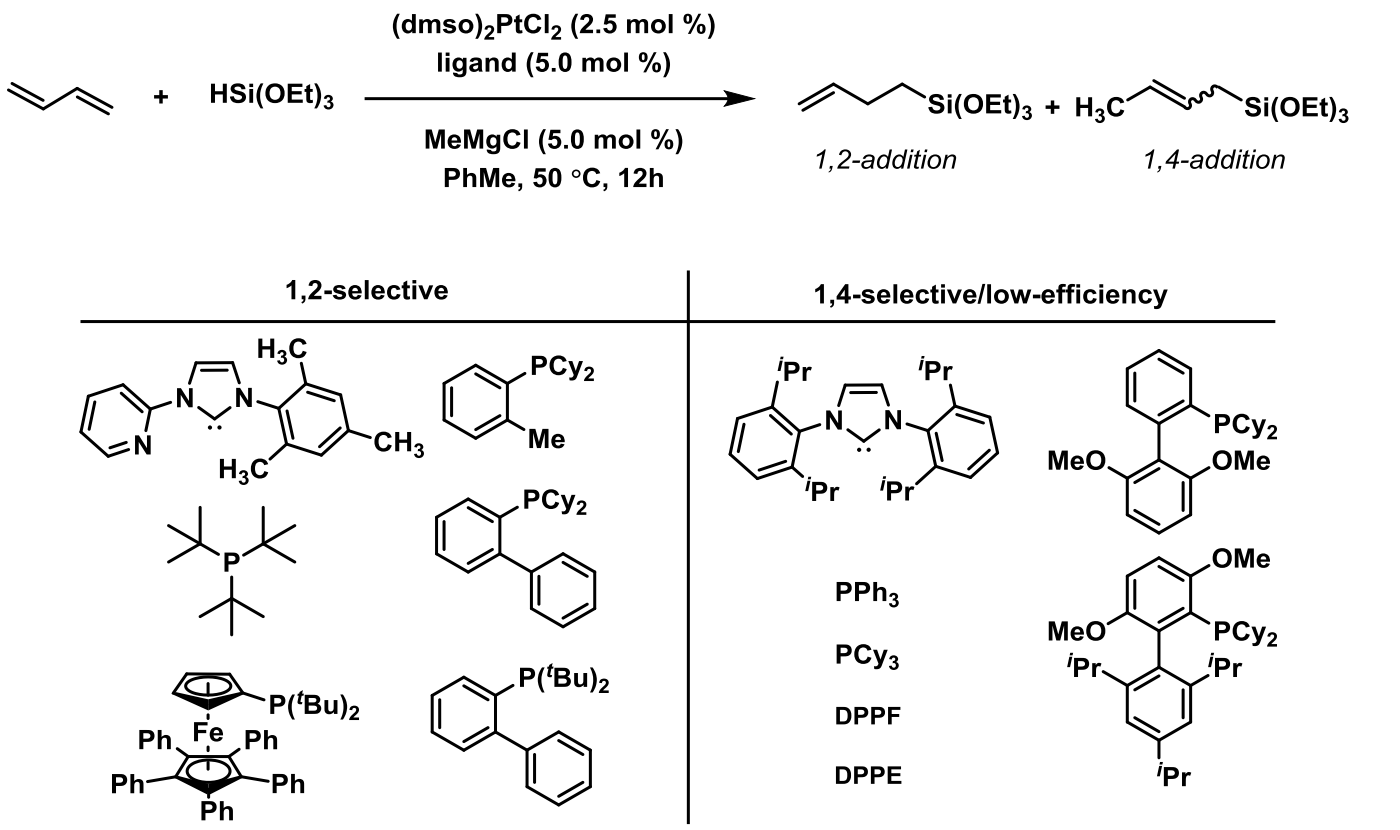

Late transition metals such as palladium and platinum are known to insert into $\mathrm{C}-\mathrm{H}$ bonds of coordinated ligands to form 4, 5, or 6-membered metallacycles, a process called cyclometallation. ${ }^{10,11}$ The mechanism by which cyclometallation occurs has not been rigorously established but $\mathrm{C}-\mathrm{H}$ cleavage is thought to occur by oxidative addition, electrophilic substitution mechanisms, or through a multi-centered $\sigma$-bond metathesis depending on the nature of the metal and the ligand. ${ }^{12}$ For platinum complexes, most

${ }^{10}$ For seminal work, see: (a) Kleiman, J. P.; Dubeck, M. J. Am. Chem. Soc. 1963, 85, 1544. (b) Cope, A. C.; Siekman, R. W. J. Am. Chem. Soc. 1965, 87, 3272. (c) Trofimenko, S. Inorg. Chem. 1973, 12, 1215.

${ }^{11}$ For reviews of cyclometallation, see: (a) Newkome, G. R.; Puckett, W. E.; Gupta, V. K.; Kiefer, G. E. Chem. Rev. 1986, 86, 451. (b) Constable, E. C. Polyhedron 1984, 3, 1037. (c) Dupont, J.; Consorti, C. S.; Spencer, J. Chem. Rev. 2005, 105, 2527. (d) Albrecht, M. Chem. Rev. 2010, 110, 576. (e) Mueller, C.; Broeckx, L. E. E.; De Krom, I.; Weemers, J. J. M. Eur. J. Inorg. Chem. 2013, 2013, 187.

${ }^{12}$ For reviews of cyclometallation mechanism studies, see: (a) Parshall, G. W. Acc. Chem. Res. 1970, 3, 139. (b) Ryabov, A. D. Chem. Rev. 1990, 90, 403. (c) Canty, A. J.; Van Koten, G. Acc. Chem. Res. 1995, $28,406$. 
cyclometallation events are thought to proceed through an agostic interaction between the metal and the $\mathrm{C}-\mathrm{H}$ bond ${ }^{13}$ followed by $\mathrm{C}-\mathrm{H}$ oxidative addition and proton removal by reductive elimination $(\mathrm{H}-\mathrm{X}, \mathrm{H}-$ $\mathrm{R}$ ) or deprotonation (Scheme 18). ${ }^{12 \mathrm{~b}, 14}$ Cyclometallation is slow at $\mathrm{C}-\mathrm{H}$ bonds that are sterically hindered, so primary $\mathrm{C}-\mathrm{H}$ bonds react faster than secondary or tertiary $\mathrm{C}-\mathrm{H}$ bonds. However, bulk elsewhere in the ligand structure accelerates metallation by forcing other $\mathrm{C}-\mathrm{H}$ bonds close to the metal center. The formation of 5-membered platinacycles is often kinetically preferred, but 4- and 6-membered metallacycles are known at a variety of metals. ${ }^{11}$

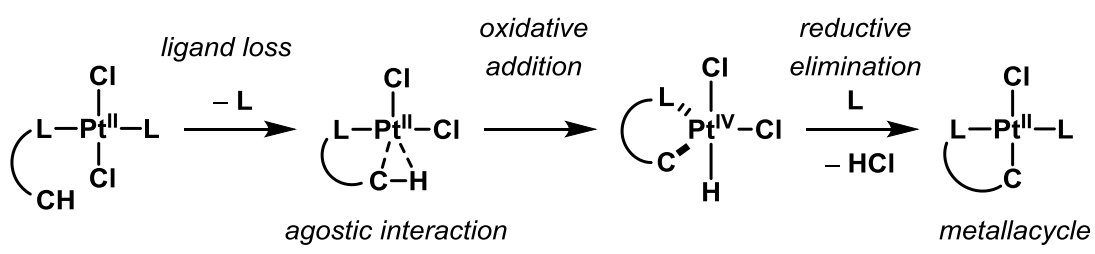

\section{Scheme 18. Mechanism of Cyclometallation at Pt(II) Complexes}

The ligands shown in Table 3 that demonstrated 1,2-selectivity in the hydrosilylation of butadiene exhibit accessible primary or aryl $\mathrm{C}-\mathrm{H}$ bonds $3-5$ bonds away from the $\sigma$-donor atom. Their similar counterparts that showed 1,4-selective hydrosilylation are hindered or substituted at the same positions, which either removes the necessary $\mathrm{C}-\mathrm{H}$ bond from the ligand entirely or creates steric hindrance that would slow the cyclometallation process. As an example, consider the similar ligands tricyclohexylphosphine and tri-tert-butylphosphine. Both phosphines are bulky and electron rich. However, tri-tert-butylphosphine contains primary $\mathrm{C}-\mathrm{H}$ bonds while tricyclohexylphosphine only contains secondary and tertiary $\mathrm{C}-\mathrm{H}$ bonds. Complexes of $\mathrm{Pt}(\mathrm{II})$ with tri-tert-butylphosphine are known

\footnotetext{
${ }^{13}$ Omae, I. J. Organomet. Chem. 2011, 696, 1128.

${ }^{14}$ For mechanism studies of C-H activation at $\mathrm{Pt}(\mathrm{II})$, see (a) Stahl, S.; Labinger, J. A.; Bercaw, J. E. Angew. Chem., Int. Ed. 1998, 37, 2181. (b) Wik, B. J.; Lersch, M.; Tilset, M. J. Am. Chem. Soc. 2002, 124, 12116. and references therein.
} 
to cyclometallate rapidly, ${ }^{15}$ while, to our knowledge, no examples of tricyclohexylphosphine cyclometallated by platinum have been reported.

To test our hypothesis that cyclometallation was the critical event leading to 1,2-selective hydrosilylation, we prepared cyclometallated complexes of $\mathrm{Pt}(\mathrm{II})$ with tri-tert-butylphosphine that contained one or two phosphine ligands ${ }^{15 b}$ and tested them as precatalysts for the hydrosilylation of butadiene. Complex 16 was more selective for 1,2-addition than the in situ generated catalyst and gave higher yields of hydrosilylation products, while complex 17, bearing two phosphine ligands, was completely unreactive. These data suggest that cyclometallation onto the phosphine ligand is beneficial for 1,2-hydrosilylation, and that the second equivalent of phosphine ligand used for in situ catalyst formation acts as a base to facilitate cyclometallation, rather than as a second ligand for platinum.

15 For early examples of cyclometallated tri-tert-butylphosphine complexes, see: (a) Goel, R. G.; Montemayor, R. Inorg. Chem. 1977, 16, 2183. (b) Clark, H. C.; Goel, A. B.; Goel, R. G.; Goel, S. Inorg. Chem. 1980, 19, 3220. (c) Goel, A. B.; Goel, S.; Vanderveer, D. Inorganica Chimica Acta-Letters 1981, 54, L267. (d) Goel, R. G.; Ogini, W. O.; Srivastava, R. C. Organometallics 1982, 1, 819. 

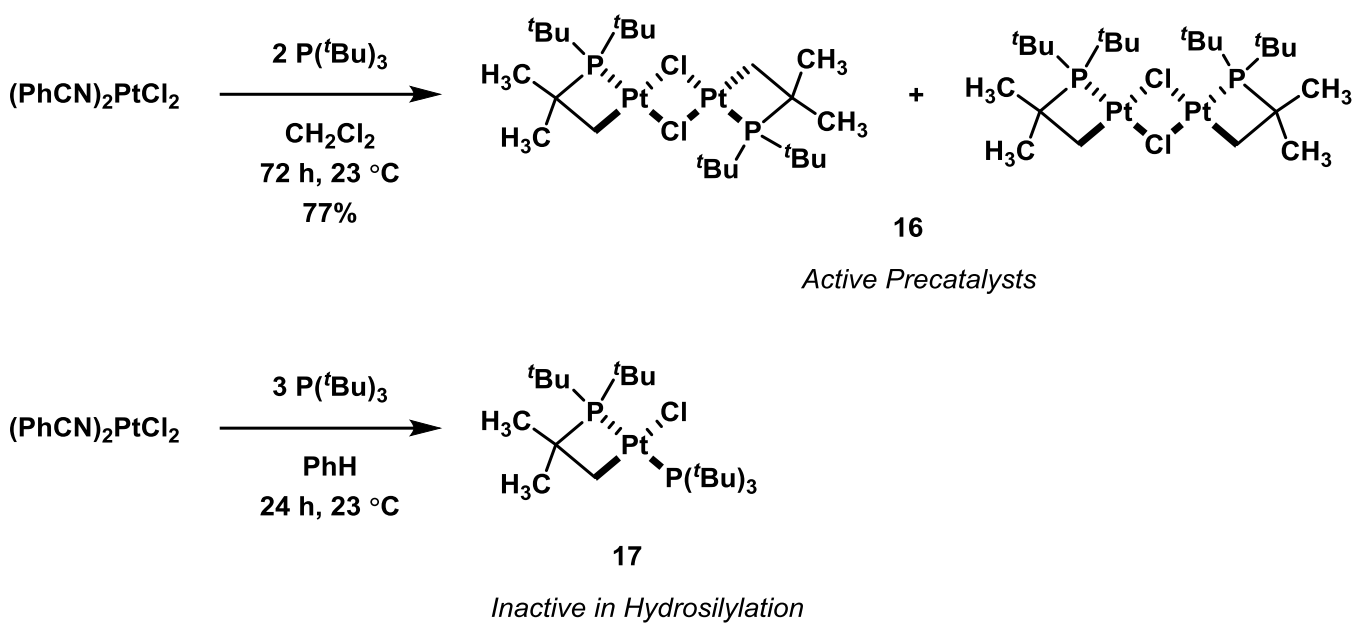

\section{Scheme 19. Synthesis of Cyclometallated Precatalysts for the Hydrosilylation of Butadiene}

\subsubsection{Hydrosilylation of 1,3-Dienes by Cyclometallated Platinum Complexes}

Upon activation with methylmagnesium chloride, cyclometallated platinum complex 16 catalyzed the selective 1,2-hydrosilylation of butadiene with 10:1 selectivity for 1,2-addition (Eq. 50) and a turnover frequency (TOF) of $480 / \mathrm{h}$ at $50{ }^{\circ} \mathrm{C}$.

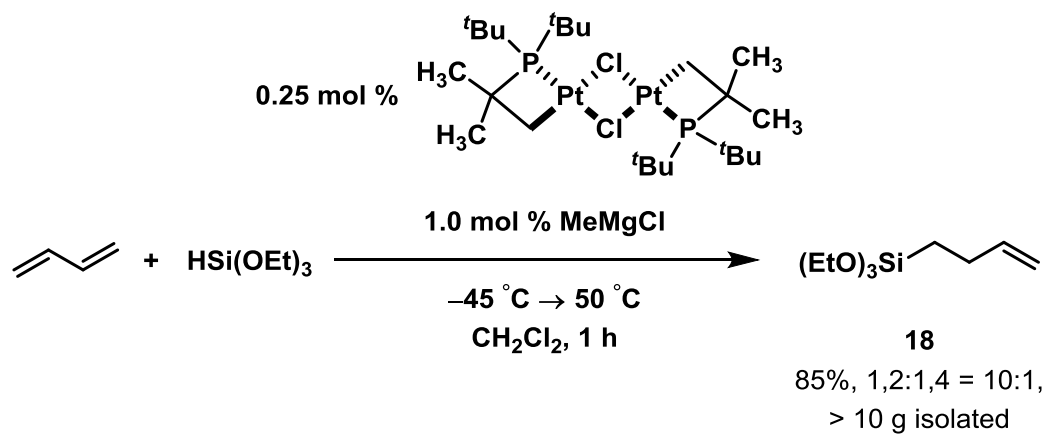

Precatalyst 16 is air- and moisture-stable and can be used for catalysis without special purification. Activation of precatalyst 16 with $\mathrm{MeMgCl}$ was performed at $-45{ }^{\circ} \mathrm{C}$ in dichloromethane, at which temperature transmetallation from the Grignard reagent proceeds more quickly than reaction with solvent. For hydrosilylation, diene and silane were added to the pre-activated catalyst solution and the vessel was sealed and heated to $50{ }^{\circ} \mathrm{C}$ until the reaction was complete. Butenylsilane products (18) were isolated by distillation of the reaction mixture without additional purification. 
Hydrosilylation using precatalyst 16 was also selective for 1,2-addition of triethoxysilane to other 1,3-dienes including isoprene, myrcene, and 2,3-dimethylbutadiene (Table 4). ${ }^{16}$ A pre-activated form of the catalyst (19, vide infra) was found to give higher yields in the hydrosilylation of substituted dienes. This difference may result from more efficient catalyst activation in the presence of butadiene than in the presence of substituted dienes. ${ }^{17}$ Selectivity for 1,2-addition increases with the bulk of the diene substituents, ranging from 10:1 (butadiene) to 90:1 (2,3-dimethylbutadiene). However, steric hindrance at the 1,3-diene results in lower conversion to hydrosilylation products at $50{ }^{\circ} \mathrm{C}$ and temperatures up to 125 ${ }^{\circ} \mathrm{C}$ are necessary to achieve full conversion with substituted dienes. Presumably, increasing the steric bulk around the 1,3-diene slows the rate of diene association to the platinum complex and leads to a greater proportion of catalyst decomposition.

\footnotetext{
${ }^{16}$ Data for substrate hydrosilylation was obtained with the help of visiting graduate student Jonas Börgel.

${ }^{17}$ We have observed that the reaction of $\mathbf{1 6}$ with methylmagnesium chloride and triethoxysilane to form 19 (vide infra) is cleaner in the presence of butadiene than in its absence, although the butadiene is not incorporated into the product (19). Combined with the observation that $\mathbf{1 9}$ is a more efficient catalyst for substituted dienes than in situ activated 16, these data suggest that catalyst activation benefits from the presence of an unhindered diene, and that substituted dienes are not able to confer this benefit as strongly as butadiene.
} 
Table 4. 1,2-Selective Hydrosilylation of Substituted Dienes

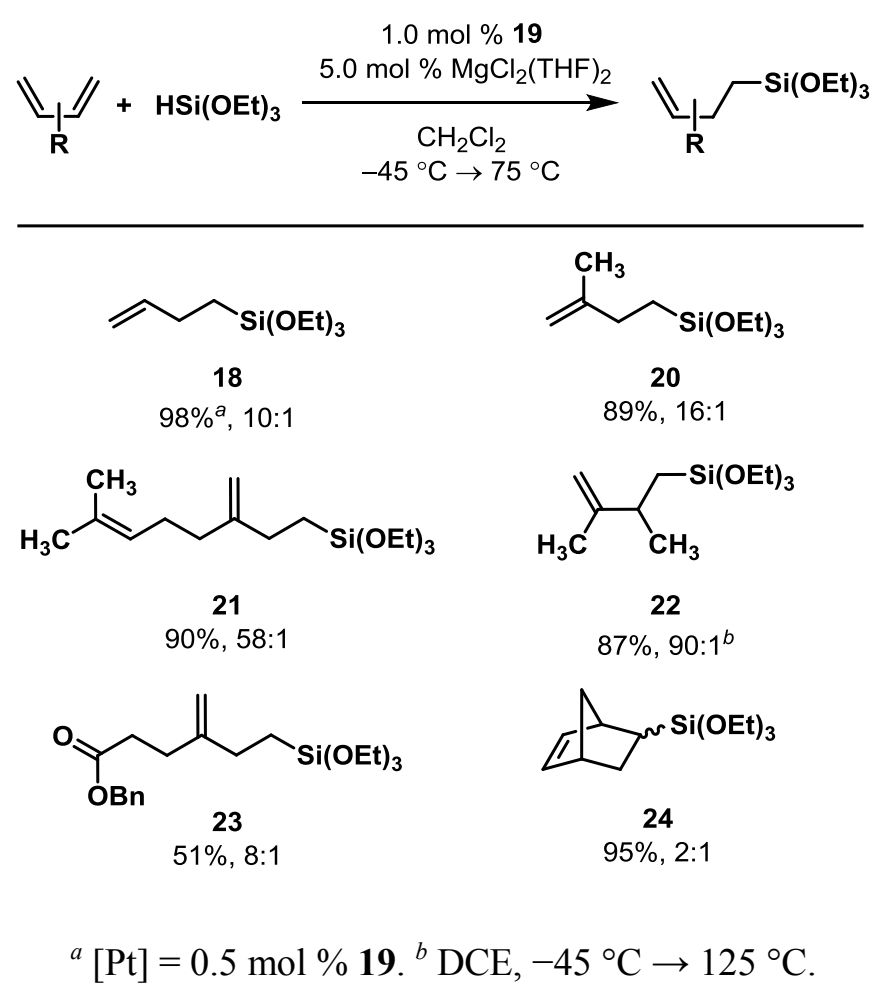

Hydrosilylation of unsymmetrically substituted dienes primarily yields the product of 1,2addition to the less-hindered olefin of the diene, but site selectivity in this case is not perfect ( $\sim 9: 1$ for isoprene, $\sim 13: 1$ for myrcene). The ratio of addition to the unsubstituted:substituted olefin also changes with the identity of the precatalyst used in hydrosilylation. Hydrosilylation of isoprene gave a 17:1 ratio using precatalyst 16 (and lower overall yield) but only 9:1 using 19, which suggests that catalyst activation is a crucial determining step for product outcomes (Table 5). 
Table 5. Comparison of Precatalysts 16 and 19 for Hydrosilylation of Butadiene

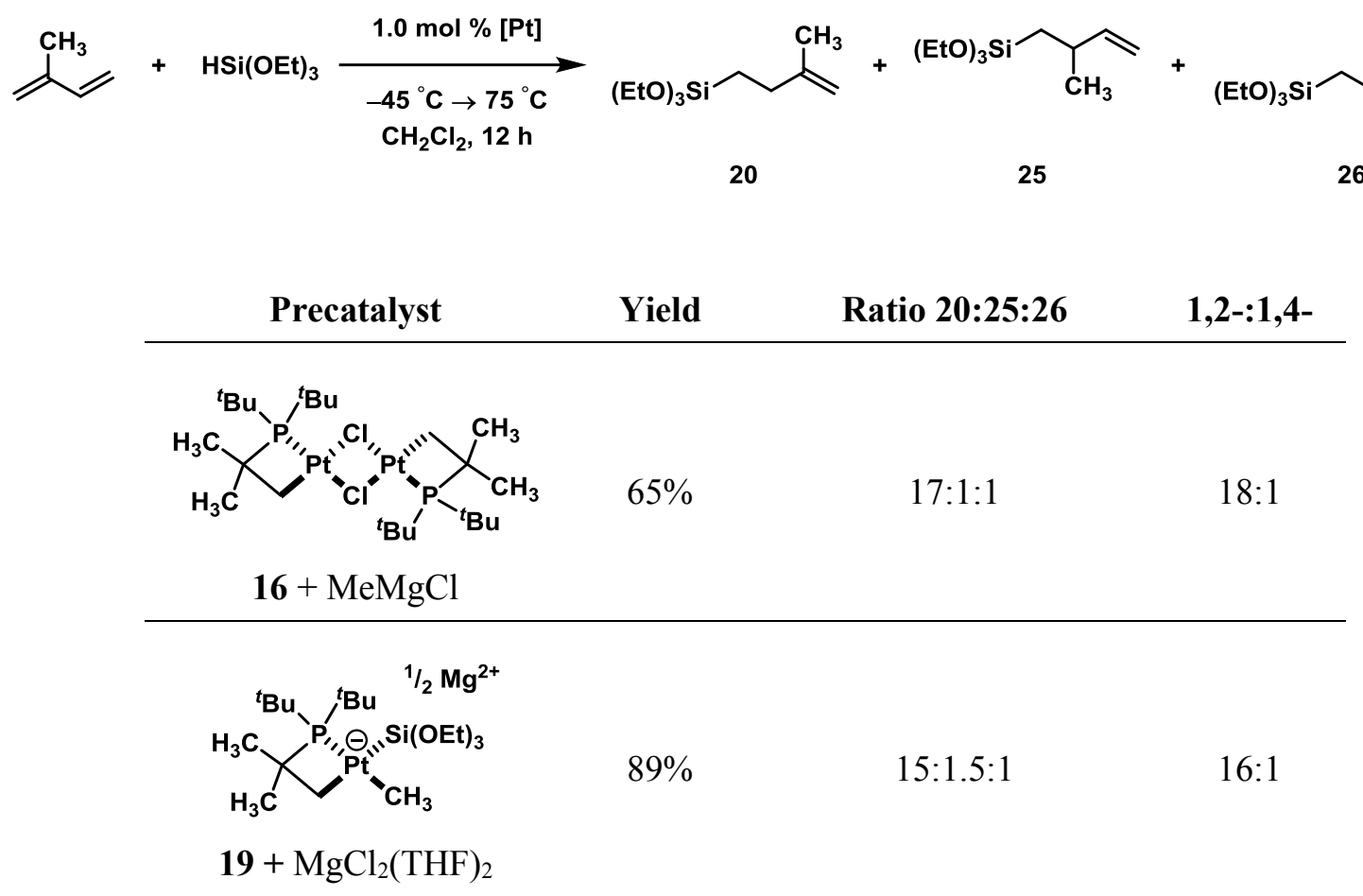

The substrates in Table 4 display few functional groups, and accordingly, functional group tolerance is low. Many Lewis basic groups (amines, sulfides, pyridines, etc.) shut down catalysis, likely by binding to either the platinum catalyst or its counterion (see section 2.3.3 below). Functional groups such as esters (23) do not interfere with catalysis, but hydrosilylation of the carbonyl group competes with hydrosilylation of the diene to form an additional byproduct (Eq. 51).

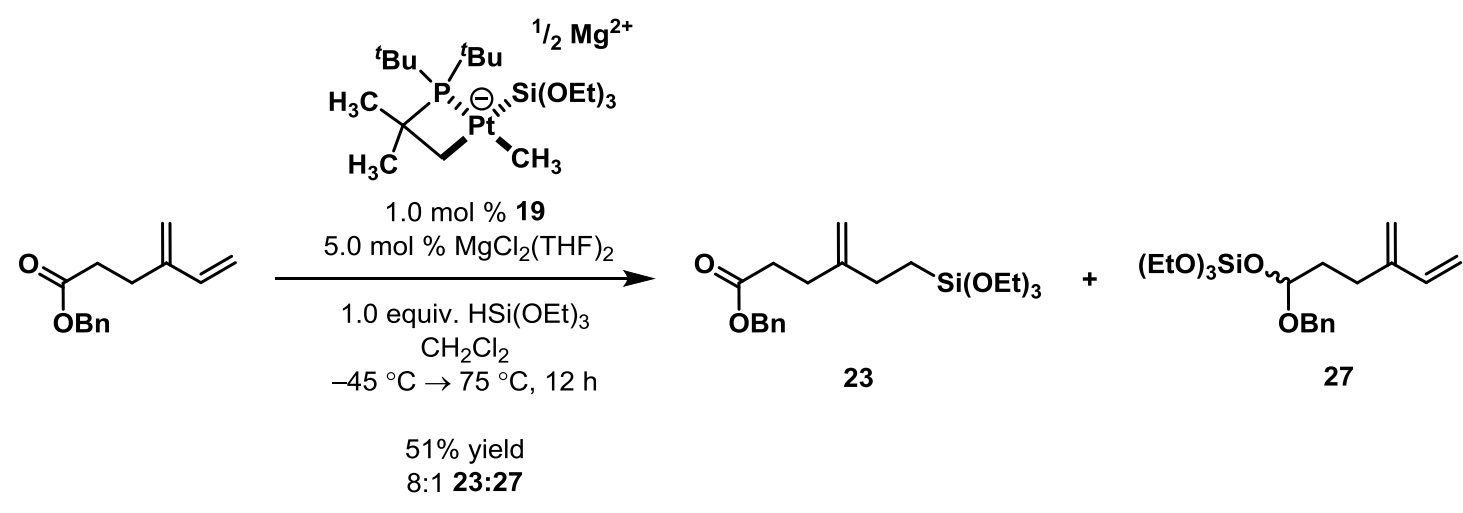

(Eq. 51) 
Norbornadiene is not a conjugated 1,3-diene, but is a difficult substrate for hydrosilylation using most catalysts. The angle strain of the [2,2,1]-bicycle is relieved by hydrosilylation of one or both double bonds because the relative difference from the ideal bond angle for an $\mathrm{sp}^{2}$-carbon when constrained in the $[2,2,1]$-bicycle is greater than the relative difference for an $\mathrm{sp}^{3}$-carbon. The angle strain present in the norbornadiene substrate results in a lower kinetic barrier for addition to the olefins of norbornadiene than to other non-conjugated dienes, and many transition metal hydrosilylation catalysts are not able to select for monoaddition. Precatalyst $\mathbf{1 6}$ adds triethoxysilane to norbornadiene once, in high overall yield (95\%), but produces a tricyclic byproduct in roughly equal proportions to the desired monoaddition product (Eq. 52). The nortricylene product is a known byproduct in the hydrosilylation of norbornadiene by platinum catalysts. $^{18}$
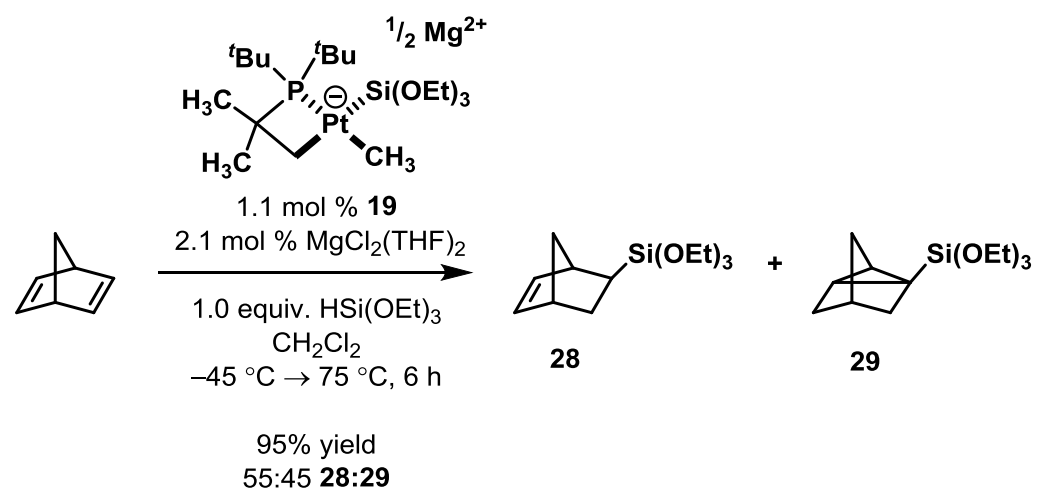

Surprisingly, the hydrosilylation of 1,3-trans-pentadiene with precatalyst 19 was not selective for 1,2-addition, but instead yielded mainly 1,4-hydrosilylation products (Eq. 53). Pentadiene is the only substrate that underwent hydrosilylation but was not selective for 1,2-addition. This result is especially surprising given that one of the few reported examples of 1,2-hydrosilylation of conjugated dienes reports cis- and trans-1,3-pentadiene as the only substrate for which 1,2-selective addition is observed. ${ }^{19}$ We do not know why we observe 1,4-addition in the case of 1,3-pentadiene, but we speculate that the addition of

\footnotetext{
${ }^{18}$ Kuivila, H. G.; Warner, C. R. J. Org. Chem. 1964, 29, 2845.

${ }^{19}$ Fink, W. Helv. Chim. Acta 1971, 54, 1304.
} 
triethoxysilane may initially occur in a 1,2-fashion and that the 1,4-product is generated by rapid isomerization, perhaps by another catalytically-active platinum species in solution. ${ }^{20}$

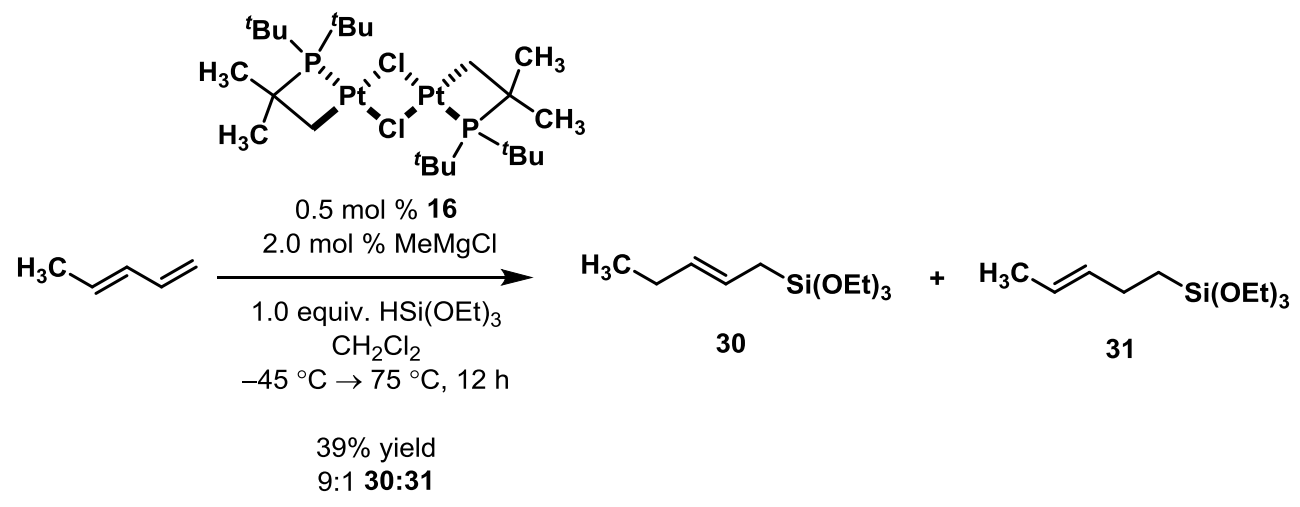

(Eq. 53)

Many substrates were inactive in hydrosilylation catalyzed by platinate $\mathbf{1 9}$. We found that styrene derivatives are prone to polymerization under the conditions of hydrosilylation, and catalyst deactivation was rapid, resulting in low overall conversion and a mixture of products. Steric hindrance of the 1,3-diene slowed down the rate of hydrosilylation and substituted dienes required elevated temperatures to reach full conversion. Other substrates, such as nopadiene, were too hindered to reach full conversion even at $125{ }^{\circ} \mathrm{C}$ and showed, instead, the formation of byproducts such as dehydrosilylation at elevated temperatures. Allyl chloride, a challenging substrate in olefin hydrosilylation, was reactive towards hydrosilylation using precatalysts $\mathbf{1 6}$ and $\mathbf{1 9}$, but resulted in complex mixtures of products.

\subsection{Mechanism of 1,2-Selective Hydrosilylation}

\subsubsection{Activation of Platinum Catalyst $\mathbf{1 6}$}

Activation of precatalyst $\mathbf{1 6}$ with two equivalents of methylmagnesium chloride at $-45{ }^{\circ} \mathrm{C}$ generates electron-rich, anionic Pt(II) complex 32 (Scheme 20). By ${ }^{1} \mathrm{H}$ and ${ }^{31} \mathrm{P}$ NMR spectroscopy, broad peaks resembling those of the product (32) are observed after several hours at $-45{ }^{\circ} \mathrm{C}$, which sharpen

${ }^{20}$ For an example transition metal catalyzed isomerization of hydrosilylation products, see: Mori, A.; Takahisa, E.; Nishihara, Y.; Hiyama, T. Can. J. Chem. 2001, 79, 1522. 
upon warming to $23{ }^{\circ} \mathrm{C}$. When the reaction is relatively concentrated, such as during the preparation of $\mathbf{3 2}$ for isolation, a white solid $\left(\mathrm{MgCl}_{2}(\mathrm{THF})_{2}\right)$ precipitates upon warming from $-45^{\circ} \mathrm{C}$ to $23{ }^{\circ} \mathrm{C}$. Together, these observations suggest that an aggregate of $\mathbf{3 2}$ and $\mathrm{MgCl}_{2}(\mathrm{THF})_{2}$ forms at low temperature, which releases $\mathrm{MgCl}_{2}(\mathrm{THF})_{2}$ when warmed to $23^{\circ} \mathrm{C}$.

Platinate 32 reacts with triethoxysilane to give platinate 19 , presumably by oxidative addition of triethoxysilane to form a transient Pt(IV) intermediate and subsequent reductive elimination of methane (Scheme 20). Consistent with this hypothesis, we observe methane in the ${ }^{1} \mathrm{H}$ NMR spectrum of this reaction. Platinates 19 and 32 are isolable as air- and moisture-sensitive aggregates with $\mathrm{MgCl}_{2}(\mathrm{THF})_{2}$ and have been characterized by ${ }^{1} \mathrm{H},{ }^{13} \mathrm{C}$, and ${ }^{31} \mathrm{P}$ NMR spectroscopy as well as by $\mathrm{x}$-ray diffraction (19). Both 19 and 32 are competent precatalysts for 1,2-selective hydrosilylation, which indicates that these complexes are intermediates on the catalyst activation pathway.

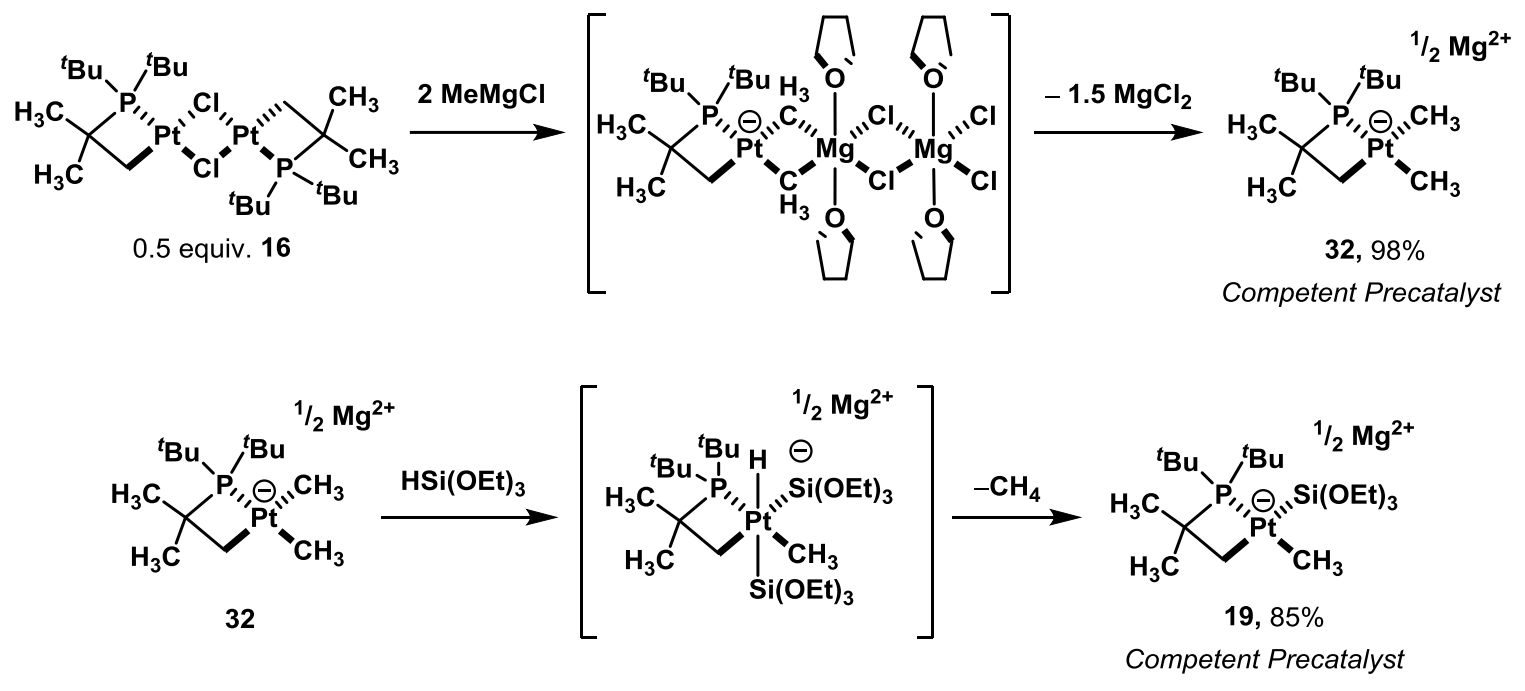

Scheme 20. Activation of Precatalyst 16

Precatalyst 19 further reacts with triethoxysilane to form as yet unidentified products (Scheme 21). We propose that precatalyst 19 further reacts with triethoxysilane by oxidative addition (E) to afford, after reductive elimination of methane (observed by GCMS), active catalyst $\mathbf{A}$. Pt(IV) intermediate $\mathbf{E}$ has a second accessible $\mathrm{C}-\mathrm{H}$ reductive elimination pathway, which releases the cyclometallated arm of the 
phosphine ligand to form monodentate tri-tert-butylphosphine and leads to catalyst decomposition (Scheme 20). Consistent with this analysis, formation of unbound $\mathrm{P}\left({ }^{t} \mathrm{Bu}\right)_{3}$ was observed during the early stages of hydrosilylation for precatalysts $\mathbf{1 6}, \mathbf{1 9}$, and $\mathbf{3 2}$.

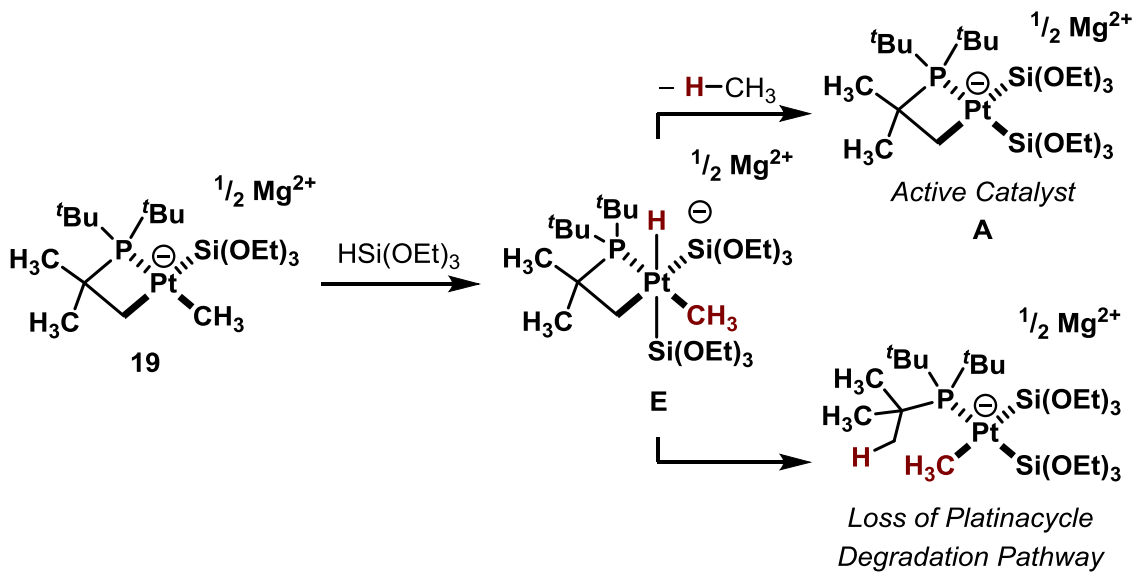

\section{Scheme 21. Proposed Mechanism of Catalyst Activation and Degradation}

\subsubsection{Proposed Mechanism for 1,2-Selective Hydrosilylation}

We propose a $\mathrm{Pt}(\mathrm{II} / \mathrm{IV})$ catalytic cycle to rationalize the observed selectivity for 1,2-addition (Scheme 22). ${ }^{21}$ Catalyst activation provides an electron-rich anionic Pt(II) complex (A) that can undergo oxidative addition of triethoxysilane to form coordinatively saturated $\mathrm{Pt}(\mathrm{IV})$ intermediate $\mathbf{B}$. Because $\mathbf{B}$ is coordinatively saturated, a ligand must dissociate prior to diene coordination (C). Importantly, because the phosphine contained in the bidentate ligand is the only ligand which can readily dissociate from $\mathbf{B}$, the incoming diene is restricted to $\eta^{2}$-coordination. 1,2-Migratory insertion forms linear alkyl complex $\mathbf{D}$, which may dissociate a ligand (such as the phosphine) to form a pentacoordinate complex prior to reductive elimination of the desired 1,2-hydrosilylation product and regeneration of $\mathbf{A}$.

${ }^{21}$ For a mechanism study of a Pt(II)/Pt(IV) catalytic cycle for hydrosilylation, see: Roy, A. K.; Taylor, R. B. J. Am. Chem. Soc. 2002, 124, 9510. 


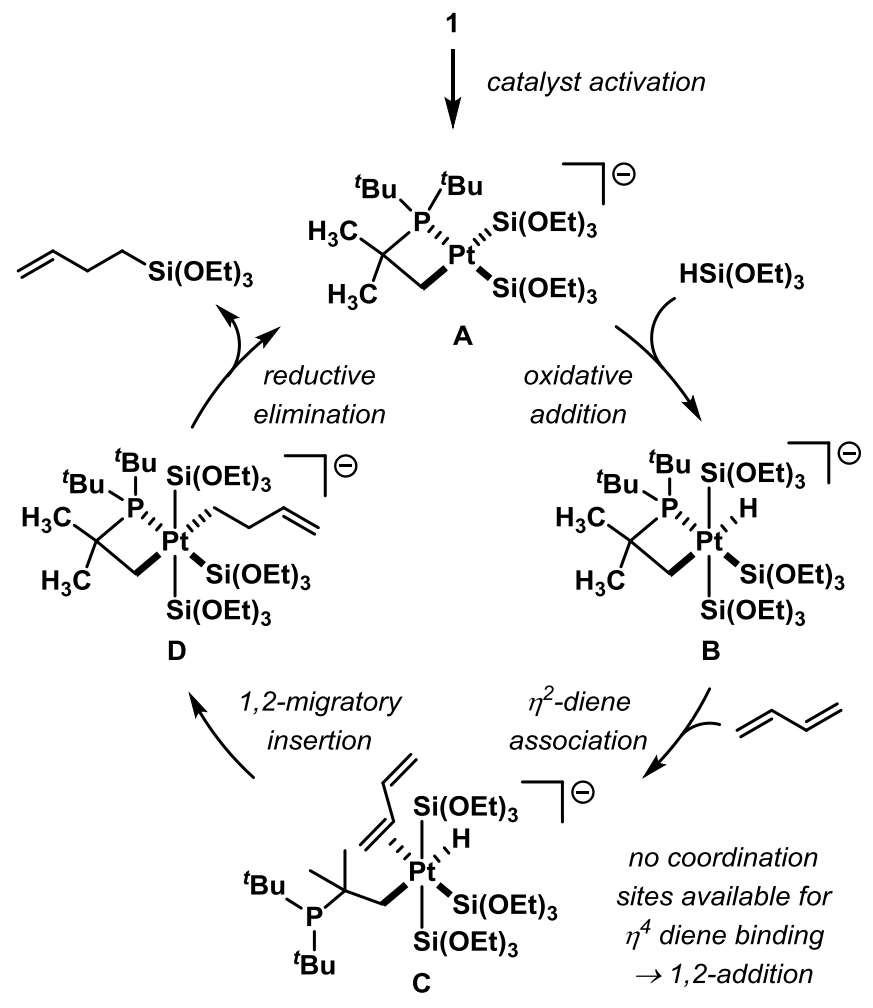

Scheme 22. Proposed Mechanism of 1,2-Selective Hydrosilylation

Oxidative addition to anionic platinate $\mathbf{A}$ is predicted to be slow, both because $\mathbf{A}$ is fourcoordinate in a square planar geometry ${ }^{22}$ and because oxidative addition of $\mathrm{Pt}(\mathrm{II})$ to the +IV oxidation state is relatively difficult compared to oxidation of $\mathrm{Pt}(0)$ to $\mathrm{Pt}(\mathrm{II})$. Experiments with related platinates 19 and 32 demonstrated that oxidative addition is slow and is only observed for relatively oxidizing reagents, such as triethoxysilane (Table 6). Other silanes such as triethyl-, dimethylphenyl-, and triphenylsilane did not react with 32 at $50{ }^{\circ} \mathrm{C}$. Another common oxidant, pinacolatodiboron, reacted only slowly and the product mixture showed only decomposition to unbound tri-tert-butylphosphine. All reactions with 32 resulted in decomposition to free tri-tert-butylphosphine except that of triethoxysilane, which forms precatalyst 19 (Scheme 20). We propose that the magnesium counterion of $\mathbf{3 2}$ coordinates to the oxygen

\footnotetext{
${ }^{22}$ Oxidative addition to square planar group 10 metals is thought to be slower than oxidative addition to T-shaped three-coordinate complexes. See: (a) Wick, D. D.; Goldberg, K. I. J. Am. Chem. Soc. 1997, 119, 10235. (b) Peters, R. G.; White, S.; Roddick, D. M. Organometallics 1998, 17, 4493.
} 
atoms of triethoxysilane, which increases the electrophilicity of the $\mathrm{Si}-\mathrm{H}$ bond and facilitates oxidative addition (see section 2.3.3).

Table 6. Reactivity of 32 with Oxidants

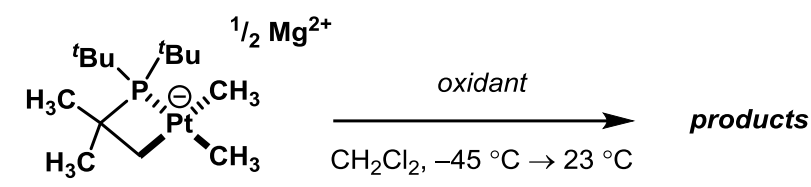

\begin{tabular}{cc} 
Oxidant & Products \\
\hline $\mathrm{HSi}(\mathrm{OEt})_{3}$ & $\mathbf{1 9}$ \\
\hline $\mathrm{HSiEt}_{3}$ & $($ no reaction $)$ \\
\hline $\mathrm{HSiPhMe}_{2}$ & $($ no reaction $)$ \\
\hline $\mathrm{HSiPh}_{3}$ & $($ no reaction $)$ \\
\hline $\mathrm{HSiCl}_{3}$ & $\begin{array}{c}\mathrm{P}\left({ }^{t} \mathrm{Bu}\right)_{3} \\
(\text { decomposition })\end{array}$ \\
\hline$(\mathrm{PinB})_{2}$ & $\begin{array}{c}\mathrm{P}\left({ }^{t} \mathrm{Bu}\right)_{3}+\mathbf{3 2} \\
(\text { partial decomposition })\end{array}$
\end{tabular}

From intermediate B (Scheme 22), phosphine dissociation would result in a coordinatively unsaturated $\mathrm{Pt}(\mathrm{IV})$ anion that can coordinate a molecule of butadiene. Importantly, all other ligands coordinated to platinum in intermediate $\mathbf{C}$ are non-dissociable, which restricts the diene to $\eta^{2}$ coordination. Migratory insertion of the coordinated diene could proceed into the $\mathrm{Pt}-\mathrm{H}$ bond, the $\mathrm{Pt}-\mathrm{C}$ bond, or one of the Pt-Si bonds. As discussed in section 1.2.3 of the introduction, platinum catalysts favor olefin insertion into $\mathrm{Pt}-\mathrm{H}$ and are likely to form the terminal alkyl complex by 1,2 -insertion. ${ }^{23,24}$ Complex

${ }^{23}$ For studies of migratory insertion of olefins at Pt catalysts, see: (a) Arnek, R.; Zetterberg, K. Organometallics 1987, 6, 1230. (b) Reger, D. L.; Garza, D. G.; Baxter, J. C. Organometallics 1990, 9, 873. (c) Stromberg, S.; Zetterberg, K.; Siegbahn, P. E. M. J. Chem. Soc., Dalton Trans. 1997, 4147. (d) Shiotsuki, M.; White, P. S.; Brookhart, M.; Templeton, J. L. J. Am. Chem. Soc. 2007, 129, 4058.

${ }^{24}$ For theoretical studies of migratory insertion of olefins at Pt catalysts, see: (a) Sakaki, S.; Ogawa, M.; Musashi, Y.; Arai, T. J. Am. Chem. Soc. 1994, 116, 7258. (b) Sakaki, S.; Mizoe, N.; Sugimoto, M. Organometallics 1998, 17, 2510. (c) Sakaki, S.; Mizoe, N.; Musashi, Y.; Sugimoto, M. J. Mol. Struct.: THEOCHEM 1999, 461-462, 533. (d) Sakaki, S.; Mizoe, N.; Sugimoto, M.; Musashi, Y. Coord. Chem. 
D is drawn with the phosphine ligand coordinated to platinum, forming a hexacoordinate octahedral platinum complex. We propose that coordination of the phosphine ligand could slow the reverse reaction by preventing $\beta$-hydride elimination of the linear alkyl ligand. By preventing the reverse reaction, the chances of a low-probability but thermodynamically preferred $\pi$-allyl complex forming are further reduced.

Previous work investigating the mechanism of reductive elimination from octahedral $\mathrm{Pt}(\mathrm{IV})$ complexes shows that reductive elimination from a hexacoordinate complex is likely to be much slower than from a pentacoordinate complex. ${ }^{25}$ Accordingly, we propose that dissociation of the phosphine ligand from D forms a pentacoordinate complex that undergoes reductive elimination to release butenylsilane $\mathbf{1 8}$ and regenerate active catalyst $\mathbf{A}$. This dissociation can also explain the decomposition pathway proposed in Scheme 21 because phosphine dissociation removes the stabilizing influence of the bidentate ligand and increases the likelihood that the cyclometallated carbon is removed from the metal by reductive elimination.

\subsubsection{Counterion Effect}

Our data suggest that the catalyst counterion plays an active role in catalysis. We propose that the $\mathrm{Mg}^{2+}$ counterion assists reductive elimination from electron-rich $\mathrm{Pt}(\mathrm{IV})(\mathbf{3 2} \rightarrow \mathbf{A}, \mathbf{D} \rightarrow \mathbf{A})$ by binding to

Rev. 1999, 190-192, 933. (e) Giorgi, G.; De Angelis, F.; Re, N.; Sgamellotti, A. J. Mol. Struct.: THEOCHEM 2003, 623, 277.

${ }^{25}$ For studies of reductive elimination from octahedral complexes, see: (a) Williams, B. S.; Holland, A. W.; Goldberg, K. I. J. Am. Chem. Soc. 1999, 121, 252. (b) Bartlett, K. L.; Goldberg, K. I.; Borden, W. T. J. Am. Chem. Soc. 2000, 122, 1456. (c) Crumpton, D. M.; Goldberg, K. I. J. Am. Chem. Soc. 2000, 122, 962. (d) Bartlett, K. L.; Goldberg, K. I.; Borden, W. T. Organometallics 2001, 20, 2669. (e) CrumptonBregel, D. M.; Goldberg, K. I. J. Am. Chem. Soc. 2003, 125, 9442. (f) Jensen, M. P.; Wick, D. D.; Reinartz, S.; White, P. S.; Templeton, J. L.; Goldberg, K. I. J. Am. Chem. Soc. 2003, 125, 8614. (g) Arthur, K. L.; Wang, Q. L.; Bregel, D. M.; Smythe, N. A.; O'neill, B. A.; Goldberg, K. I.; Moloy, K. G. Organometallics 2005, 24, 4624. (h) Procelewska, J.; Zahl, A.; Liehr, G.; Van, E. R.; Smythe, N. A.; Williams, B. S.; Goldberg, K. I. Inorg. Chem. 2005, 44, 7732. (i) Kloek, S. M.; Goldberg, K. I. J. Am. Chem. Soc. 2007, 129, 3460. (j) Luedtke, A. T.; Goldberg, K. I. Inorg. Chem. 2007, 46, 8496. (k) Pawlikowski, A. V.; Getty, A. D.; Goldberg, K. I. J. Am. Chem. Soc. 2007, 129, 10382. (1) Smythe, N. A.; Grice, K. A.; Williams, B. S.; Goldberg, K. I. Organometallics 2009, 28, 277. 
triethoxysilyl ligands of the Pt(IV) anion. Magnesium ions could also promote catalyst activation and/or hydrosilylation by binding to $(\mathrm{EtO})_{3} \mathrm{SiH}$ prior to oxidative addition, which would increase the electrophilicity of the $\mathrm{Si}-\mathrm{H}$ bond and facilitate oxidative addition $(\mathbf{3 2} \rightarrow \mathbf{A}, \mathbf{A} \rightarrow \mathbf{B})$. Lewis acids have been shown to promote both oxidative addition ${ }^{26}$ and reductive elimination ${ }^{25 a, 27}$ at transition metal complexes.

In situ generation of precatalyst 19 results in a visibly homogeneous solution at the concentrations used for catalysis. This solution presumably contains precatalyst 19 and one equivalent of dissolved $\mathrm{MgCl}_{2}(\mathrm{THF})_{2}$, which is produced by catalyst activation and remains dissolved during hydrosilylation. When precatalyst 19 is synthesized for isolation, higher concentrations are employed at which $\mathrm{MgCl}_{2}(\mathrm{THF})_{2}$ precipitates and is removed by filtration. Isolated precatalyst 19 shows significantly reduced activity and selectivity in the hydrosilylation of butadiene (Table 7), which suggests that the loss of additional magnesium in solution has a negative effect on both activity and selectivity in hydrosilylation. Notably, much of the loss in activity and selectivity when using isolated 19 can be recovered by adding $\mathrm{MgCl}_{2}(\mathrm{THF})_{2}$ and ligand quantities of THF (Table 7). We attribute the slight difference in yield with $19+\mathrm{MgCl}_{2}(\mathrm{THF})_{2}$ compared to the in situ generated catalyst (from $\mathbf{1 6}+2$ $\mathrm{MeMgCl})$ to the low solubility of $\mathrm{MgCl}_{2}(\mathrm{THF})_{2}$ in the chlorinated solvents used for hydrosilylation. We attempted to test this hypothesis by removing additional magnesium salts from the reaction mixture by

${ }^{26}$ For examples of Lewis acid-assisted oxidative addition, see: (a) Ghosh, I.; Jacobi, P. A. J. Org. Chem. 2002, 67, 9304. (b) Shi, Y.; Peterson, S. M.; Haberaecker, W. W., Iii; Blum, S. A. J. Am. Chem. Soc. 2008, 130, 2168. (c) Nakao, Y.; Idei, H.; Kanyiva, K. S.; Hiyama, T. J. Am. Chem. Soc. 2009, 131, 15996. (d) Nakao, Y.; Idei, H.; Kanyiva, K. S.; Hiyama, T. J. Am. Chem. Soc. 2009, 131, 5070. (e) Ohashi, M.; Shibata, M.; Saijo, H.; Kambara, T.; Ogoshi, S. Organometallics 2013, 32, 3631.

${ }^{27}$ For examples of Lewis acid-assisted reductive elimination, see: (a) Stahl, S. S.; Labinger, J. A.; Bercaw, J. E. J. Am. Chem. Soc. 1995, 117, 9371. (b) Huang, J.; Haar, C. M.; Nolan, S. P.; Marcone, J. E.; Moloy, K. G. Organometallics 1999, 18, 297. (c) Nakamura, E.; Yamanaka, M.; Mori, S. J. Am. Chem. Soc. 2000, 122, 1826. (d) Williams, B. S.; Goldberg, K. I. J. Am. Chem. Soc. 2001, 123, 2576. (e) Yamamoto, T.; Abla, M.; Murakami, Y. Bull. Chem. Soc. Jpn. 2002, 75, 1997. (f) Shen, Q. L.; Hartwig, J. F. J. Am. Chem. Soc. 2007, 129, 7734. (g) Liberman-Martin, A. L.; Bergman, R. G.; Tilley, T. D. J. Am. Chem. Soc. 2013, 135, 9612. 
adding ligand-quantities of 1,4-dioxane. Dioxane binds strongly to $\mathrm{MgX}_{2}$ salts and is reported to induce precipitation even at low concentrations. ${ }^{28}$ Addition of 1,4-Dioxane further reduces conversion in the hydrosilylation of butadiene with isolated 19 (Table 7). X-ray data show that multiple aggregates of 19 with $\mathrm{Mg}^{2+}$ salts are formed under different conditions and we postulate that the aggregation of $\mathbf{1 9}$ with additional equivalents of magnesium salts is crucial for 1,2-selectivity in hydrosilylation and for achieving an acceptable reaction rate with precatalysts 16,19 , and 32 .

To further confirm our assertion that the identity of the counterion is important for catalysis, we attempted to synthesize analogues of $\mathbf{3 2}$ with $\mathrm{Li}^{+}$and $\mathrm{K}^{+}$counterions by varying the reducing agent used for activation of 16 (Table 7). Although other Grignard reagents such as EtMgCl and $\mathrm{PhMgCl}$ show similar activity and selectivity in hydrosilylation, reducing agents with other cations such as $\mathrm{Li}^{+}$and $\mathrm{K}^{+}$ show decreased activity and markedly reduced 1,2-selectivity in the hydrosilylation of butadiene. Separate experiments have verified that $\mathrm{MeLi}$ and $\mathrm{BnK}$ transmetallate two alkyl groups to platinum, forming anionic complexes. We attribute the failure of $\mathrm{Li}^{+}$and $\mathrm{K}^{+}$cations to facilitate hydrosilylation to their oxophilicity and higher energy of solvation, which would reduce the favorability of cation interaction with the oxygen atoms of the triethoxysilyl group.

${ }^{28}$ Schlenk, W.; Schlenk, W., Jr. Ber. Dtsch. Chem. Ges. B 1929, 62B, 920. 
Table 7. Effect of Counterion on the Hydrosilylation of Butadiene

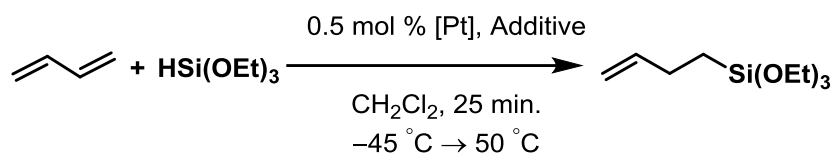

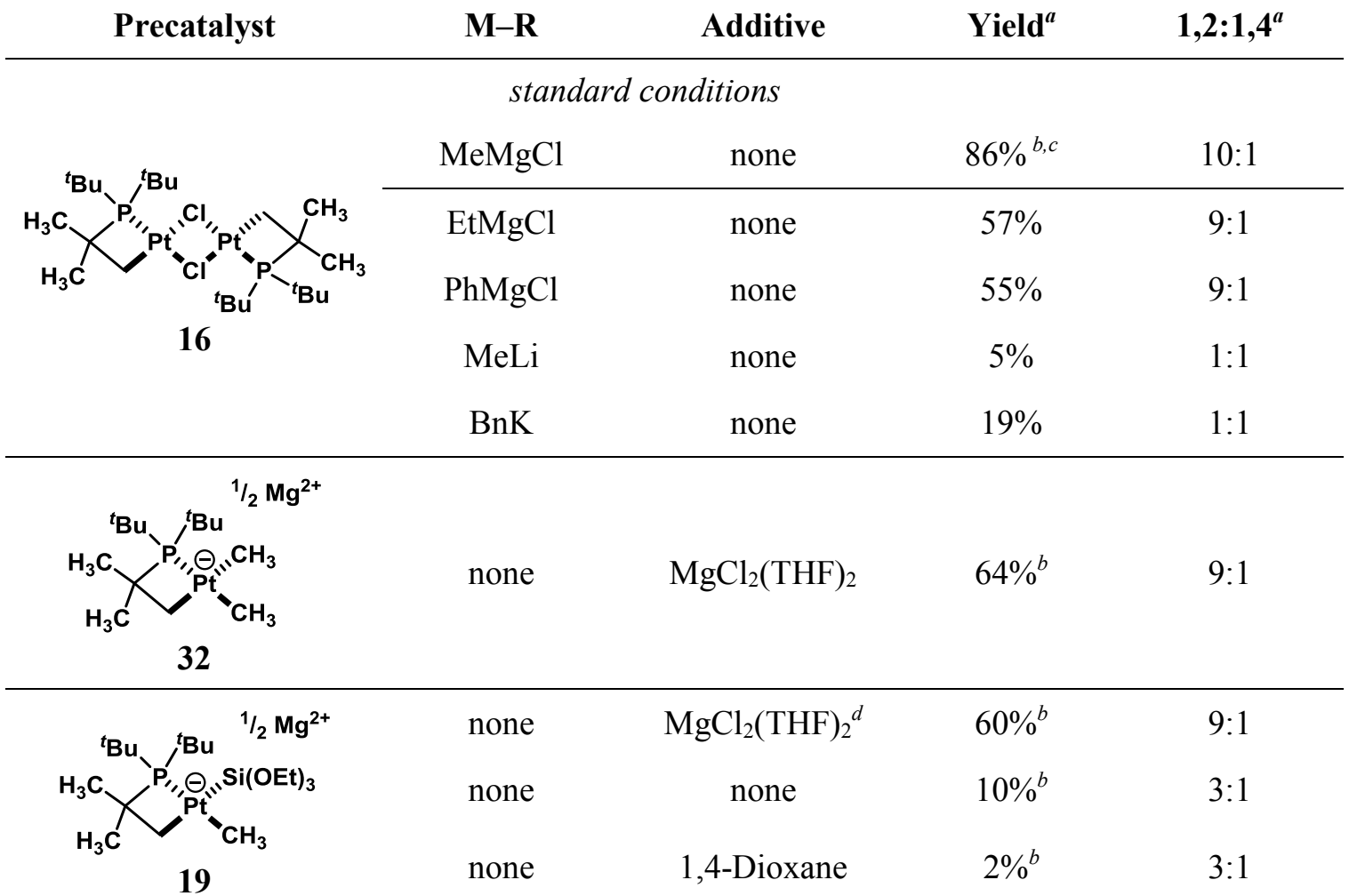

${ }^{a}$ Yield and selectivity determined by integration of $1 \mathrm{H}$ NMR resonances corresponding to the alkenyl hydrogen atoms vs. $\mathrm{TMS}_{2} \mathrm{O}$ (internal standard). ${ }^{b}$ Yield corresponds to conversion as no other products are observed. ${ }^{c}$ Isolated yield; reaction halted by $10 \mu \mathrm{L} \mathrm{N}, \mathrm{N}^{\prime}$-DMEDA after $25 \mathrm{~min} .{ }^{d}$ Addition of $\mathrm{MgCl}_{2}(\mathrm{THF})_{2}$ simulates conditions for catalysis with precatalyst 16.

X-ray diffraction data from two single crystals of $\mathbf{1 9}$ grown under different conditions show an interaction of $\mathrm{Mg}^{2+}$ with the oxygen atoms of the triethoxysilyl ligand (Figure 4, Figure 5). However, the aggregation state of 19 with additional $\mathrm{MgCl}_{2}(\mathrm{THF})_{2}$ in the two structures is different. The diffraction data shown in Figure 4 were obtained from a single crystal grown from a saturated solution of THF/pentane. Under these conditions, the counterion to the platinate of $\mathbf{1 9}$ can best be described as $\mathrm{Mg}_{2} \mathrm{Cl}_{3}(\mathrm{THF})_{3}{ }^{+}$. The diffraction data shown in Figure 5 were obtained from a crystal grown from a 
saturated solution of 19 in $\mathrm{MeCN}$. During crystallization from $\mathrm{MeCN}$, a single molecule of $\mathrm{Mg}^{2+}$ coordinates two molecules of platinate, and no additional $\mathrm{MgCl}_{2}$ salts are found. Aggregation of additional equivalents of $\mathrm{MgCl}_{2}$ (THF) $)_{2}$ to 19 may effectively tether a second Lewis acidic $\mathrm{MgCl}_{2}(\mathrm{THF})_{2}$ molecule to a molecule of catalyst. If Lewis acid activation of $(\mathrm{EtO})_{3} \mathrm{SiH}$ is necessary for efficient oxidative addition, then aggregation of additional magnesium atoms to the active species may be crucial for catalysis.

Other silanes that lack Lewis basic ligands are unreactive and unselective in hydrosilylation using precatalyst 16 (Table 8), which supports the concept of $\mathrm{Mg}^{2+}$ co-catalysis through an interaction with the oxygen atoms of triethoxysilane. Conversion increases with the electrophilicity of the silane, with the exception of triethoxysilane, which shows higher conversion than expected based on its electrophilicity. Triethoxysilane is also the only silane with Lewis basic ligands that could potentially coordinate the magnesium counterion. Triethoxysilane is also the only silane to show selectivity for 1,2-addition to butadiene, which may result from either more efficient catalyst activation or from an effect of $\mathrm{Mg}^{2+}$ on the relative rates of steps in the catalytic cycle. 

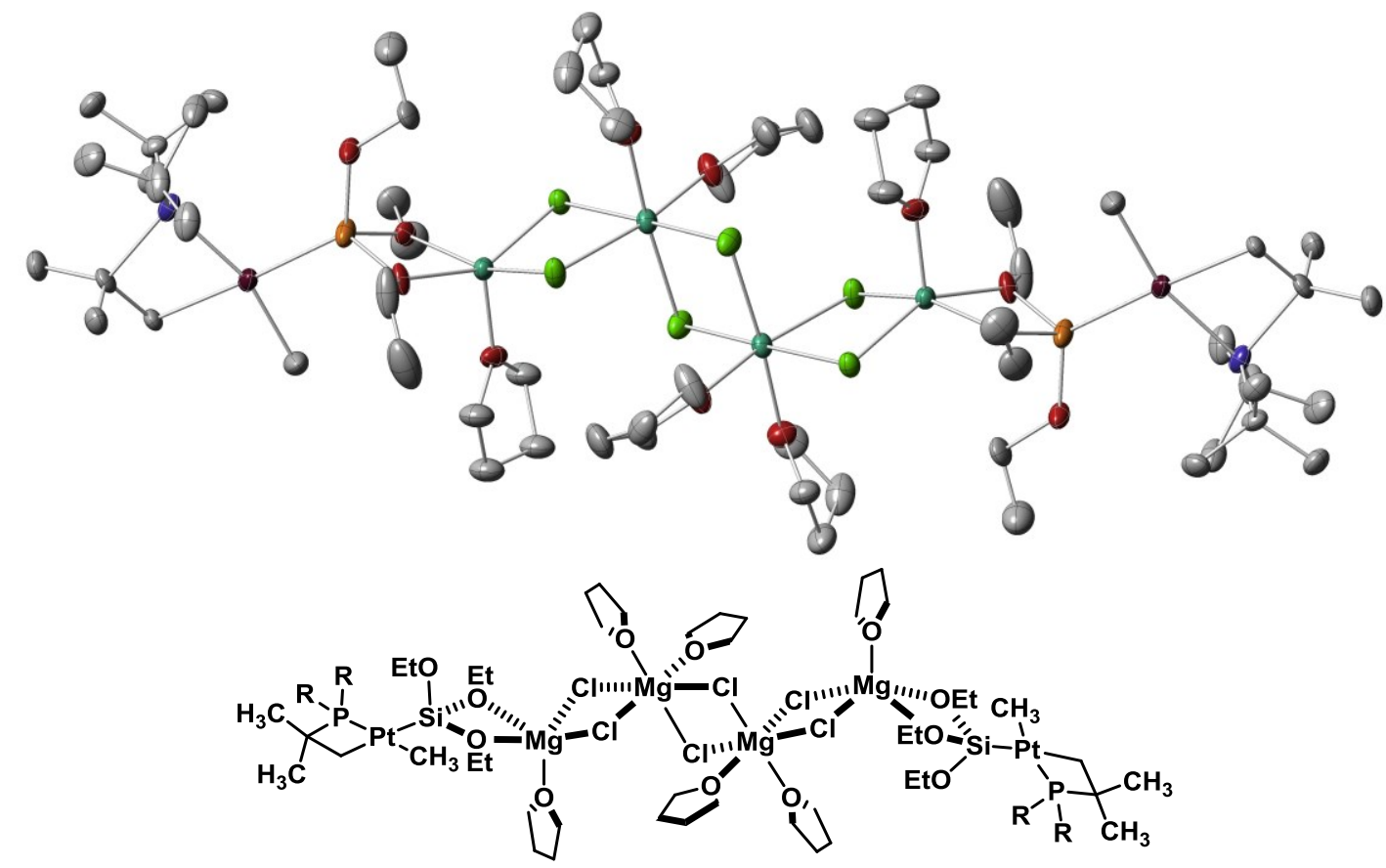

Figure 4. X-ray structure of precatalyst 19 showing aggregation with $\mathrm{MgCl}_{2}(\mathrm{THF})_{2}$. Thermal ellipsoids are drawn at the $50 \%$ probability level, H-atoms are omitted for clarity.

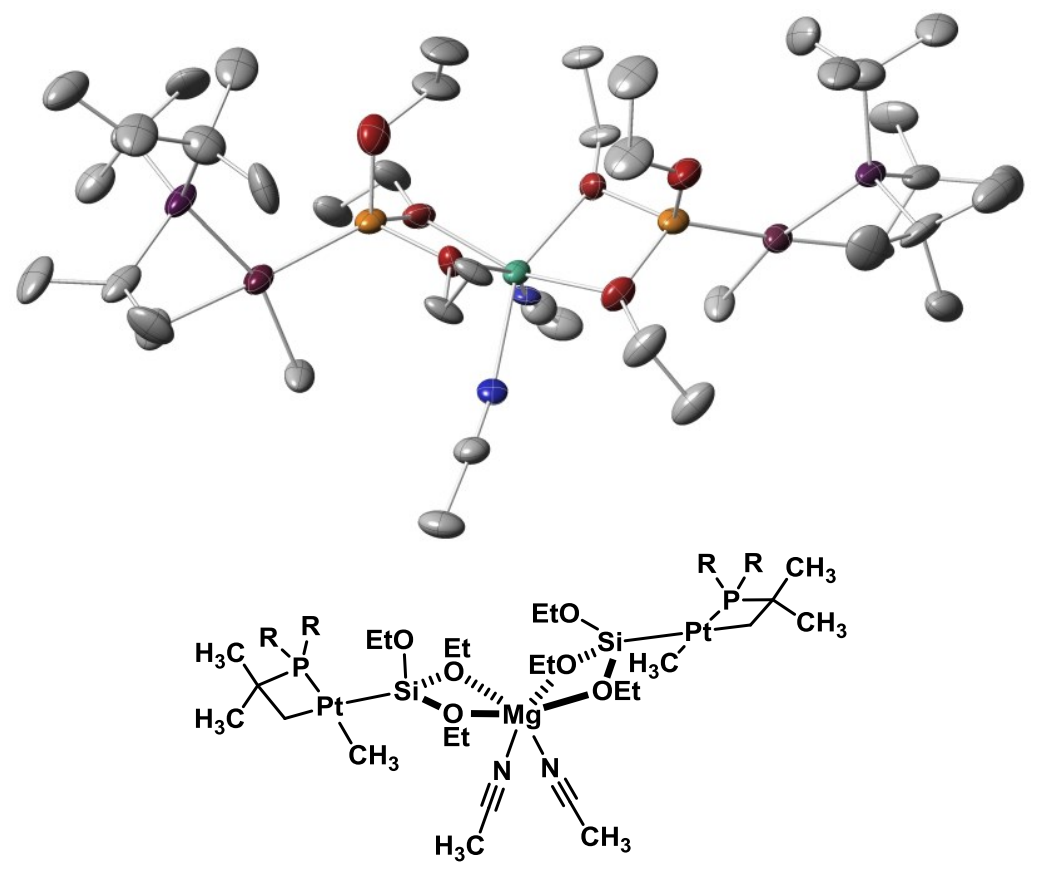

Figure 5. Second x-ray structure of precatalyst 19 showing change in counterion aggregation. Thermal ellipsoids are drawn at the $50 \%$ probability level, $\mathrm{H}$-atoms are omitted for clarity. 
Table 8. Effect of Silane on Hydrosilylation Using Precatalyst 19

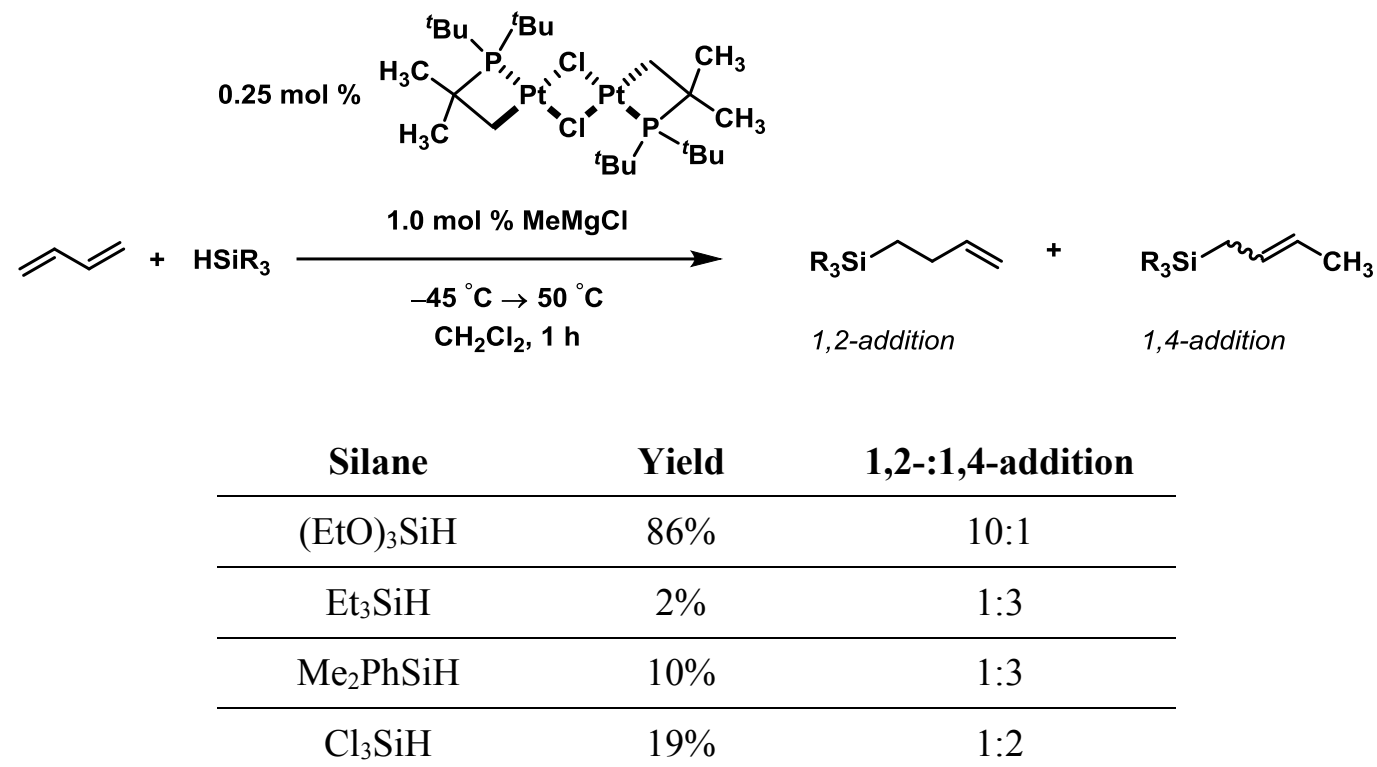

Anionic platinum(II) complexes with alkyl ligands have been reported, most commonly with ligand-centered anions (Figure 6). ${ }^{29,30}$ Reported platinates in which the platinum bears the negative charge also contain Lewis basic groups on the ligand that coordinate to the counter-cations. Stabilization of the cation is proposed to allow for the isolation and crystallization of the complexes.

\footnotetext{
${ }^{29}$ For examples with platinum-centered anions, see: (a) Poverenov, E.; Gandelman, M.; Shimon, L. J. W.; Rozenberg, H.; Ben-David, Y.; Milstein, D. Chem. - Eur. J. 2004, 10, 4673. (b) Moret, M. E.; Chen, P. Organometallics 2008, 27, 4903. (c) Poverenov, E.; Iron, M. A.; Gandelman, M.; Ben-David, Y.; Milstein, D. Eur. J. Inorg. Chem. 2010, 1991.

${ }^{30}$ For examples with ligand-centered anions, see: (a) Vedernikov, A. N.; Fettinger, J. C.; Mohr, F. J. Am. Chem. Soc. 2004, 126, 11160. (b) Vedernikov, A. N. Chem. Commun. 2009, 4781. (c) Pal, S.; Vedernikov, A. N. Dalton Transactions 2012, 41, 8116.
} 


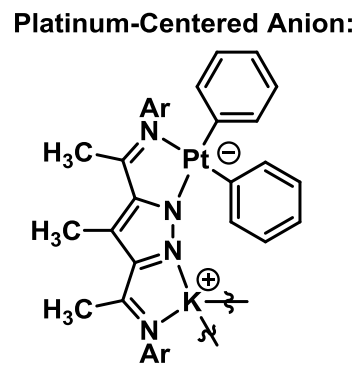

Moret and Chen, 2008

Ligand-Centered Anion

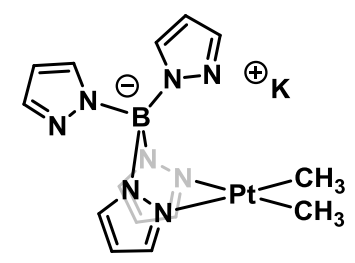

Wick and Goldberg, 1997

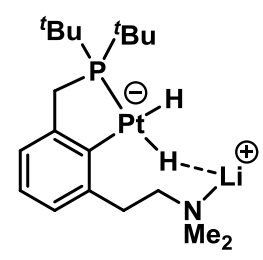

Poverenov et al., 2004

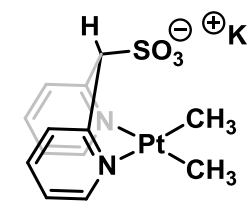

Vedernikov et al., 2004

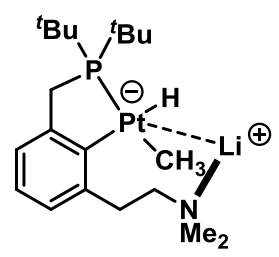

Poverenov et al., 2010

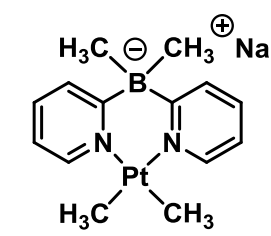

Pal and Vedernikov, 2012

Figure 6. Previously reported anionic platinum(II) complexes containing both platinum-centered and ligand-centered anions.

In the case of complexes 19 and 32, the lack of additional Lewis basic sites for counterion coordination seems to be crucial for catalytic activity. Precatalysts prepared in the presence of ligand quantities of Lewis basic molecules (for example: pyridine, $\mathrm{SMe}_{2}$ ) showed little to no conversion in the hydrosilylation of butadiene (Table 9). Because the coordination sphere of Pt(II) is effectively saturated with four ligands, it is unlikely for the additional Lewis base to be acting as a ligand for platinum. Instead, we propose that added Lewis bases interact with the $\mathrm{Mg}^{2+}$ counterion or with aggregated molecules of $\mathrm{MgCl}_{2}$. With other stabilizing ligands available, the $\mathrm{Mg}^{2+}$ or aggregated $\mathrm{MgCl}_{2}$ may not interact with the oxygen atoms of the triethoxysilyl group to provide the essential Lewis-acid co-catalysis for hydrosilylation. When few other Lewis bases are available, interaction with the relatively hindered oxygen atoms of the triethoxysilyl group may be favored.

To further test whether loss of Lewis acidity was the cause of low conversion in the presence of additional Lewis bases, we synthesized a platinate precatalyst with a non-coordinating cation (33) by precipitation of $\mathrm{MgX}_{2}$ with 1,4-dioxane in the presence of tetrabutylammonium bromide (Eq. 54). 


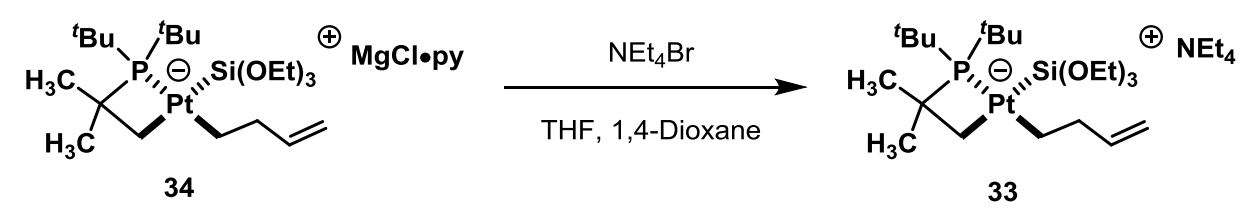

(Eq. 54)

Platinum complex $\mathbf{3 3}$ is inactive as a precatalyst for the hydrosilylation of butadiene, similar to complexes 34 and 34. However, addition of exogenous $\mathrm{MgCl}_{2}(\mathrm{THF})_{2}$ to reactions catalyzed by precatalysts lacking Lewis acidic counterions improves both the conversion and the 1,2-selectivity in the hydrosilylation of butadiene (Table 9).

Even weak Lewis bases such as acetonitrile and THF reduce conversion when present in solvent quantities. A screen of reaction solvents shows that coordinating solvents, such as THF, are poor solvents for hydrosilylation (Table 10). Extremely non-polar solvents also slow down catalysis, but we attribute this effect to poor solubility of the charged precatalysts in these solvents. 
Table 9. Performance of Catalysts Containing Additional Lewis Bases

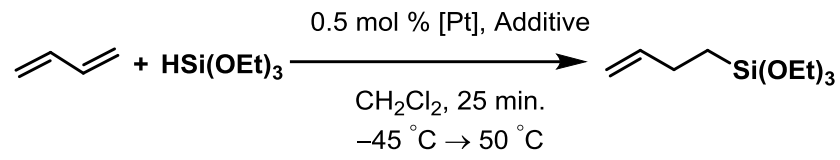

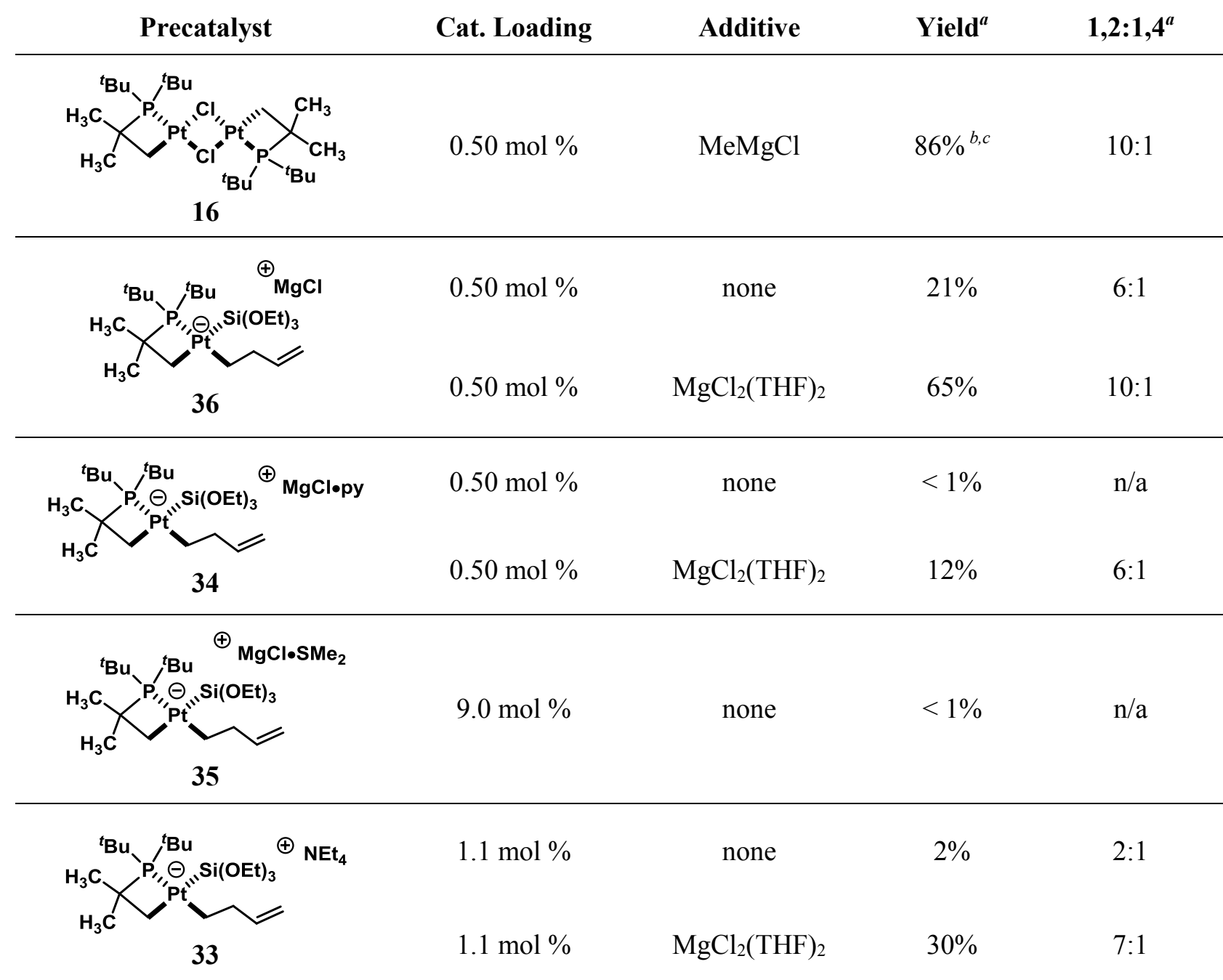

${ }^{a}$ Yield and selectivity determined by integration of ${ }^{1} \mathrm{H}$ NMR resonances corresponding to the alkenyl hydrogen atoms vs. $\mathrm{TMS}_{2} \mathrm{O}$ (internal standard). ${ }^{b}$ Yield corresponds to conversion as no other products are observed. ${ }^{c}$ Isolated yield; reaction halted by $10 \mu \mathrm{L} \mathrm{N}, \mathrm{N}^{\prime}$-DMEDA after $25 \mathrm{~min}$. 
Table 10. Effect of Solvent on 1,2-Hydrosilylation of Butadiene

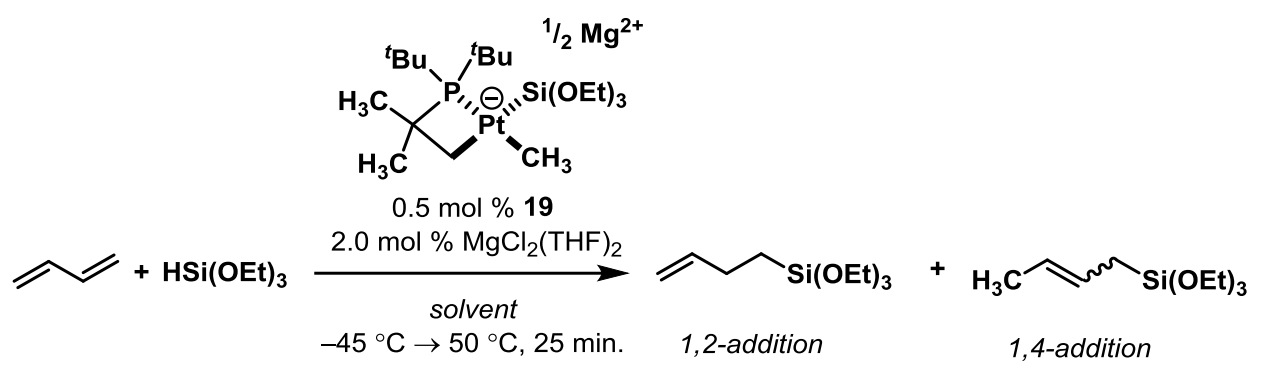

\begin{tabular}{ccc} 
Solvent & Yield & 1,2-:1,4-addition \\
\hline $\mathrm{CH}_{2} \mathrm{Cl}_{2}$ & $86 \%$ & $10: 1$ \\
\hline $\mathrm{C}_{6} \mathrm{H}_{6}$ & $43 \%$ & $7: 1$ \\
\hline $\mathrm{PhCH}_{3}$ & $37 \%$ & $5: 1$ \\
\hline $\mathrm{Et}_{2} \mathrm{O}$ & $31 \%$ & $6: 1$ \\
\hline $\mathrm{PhCN}$ & $28 \%$ & $8: 1$ \\
\hline Pentane & $9 \%$ & $3: 1$ \\
\hline $\mathrm{THF}$ & $9 \%$ & $2: 1$ \\
\hline $\mathrm{MeCN}$ & $6 \%$ & $6: 1$
\end{tabular}

\subsubsection{Platinacycle Size}

Most reported complexes containing a platinacycle are 5-membered ring platinacycles, ${ }^{31}$ which suggests that a 5-membered platinacycle is likely to be more stable than the 4-membered platinacycle in precatalysts 16, 19, and 32 . Because precatalysts 16, 19, and 32 decompose during catalyst activation, we hypothesized that 5-membered ring analogs of these precatalysts could be more stable towards decomposition during catalyst activation and, therefore, show greater activity and selectivity as catalysts for hydrosilylation of dienes. To test this hypothesis we synthesized several analogs of $\mathbf{3 2}$ containing a 5membered ring platinacycle structure. Unlike precatalyst 16, platinacycles with 5-membered rings were

${ }^{31}$ For representative examples, see: (a) Cheney, A. J.; Shaw, B. L. J. Chem. Soc., Dalton Trans. 1972, 754. (b) Gill, D. F.; Shaw, B. L. J. Chem. Soc., Chem. Commun. 1972, 65. (c) Chang, L. S.; Johnson, M. P.; Fink, M. J. Organometallics 1991, 10, 1219. (d) Ferrer, C.; Raducan, M.; Nevado, C.; Claverie, C. K.; Echavarren, A. M. Tetrahedron 2007, 63, 6306. (e) Zucca, A.; Stoccoro, S.; Cinellu, M. A.; Petretto, G. L.; Minghetti, G. Organometallics 2007, 26, 5621. 
insoluble in organic solvents as chloride-bridged dimers. To obtain complexes with higher solubility, the chlorides were abstracted with silver(I) trifluoromethanesulfonate to form cationic bis-acetonitrile complexes. From the cationic complexes, synthesis of anionic dimethylplatinate precatalysts could be achieved with methylmagnesium chloride in a straightforward manner. This synthetic pathway is shown for precatalyst $\mathbf{3 7}$ below in Scheme 23 and was also used to synthesize precatalysts $\mathbf{3 8}$ and $\mathbf{3 9}$.

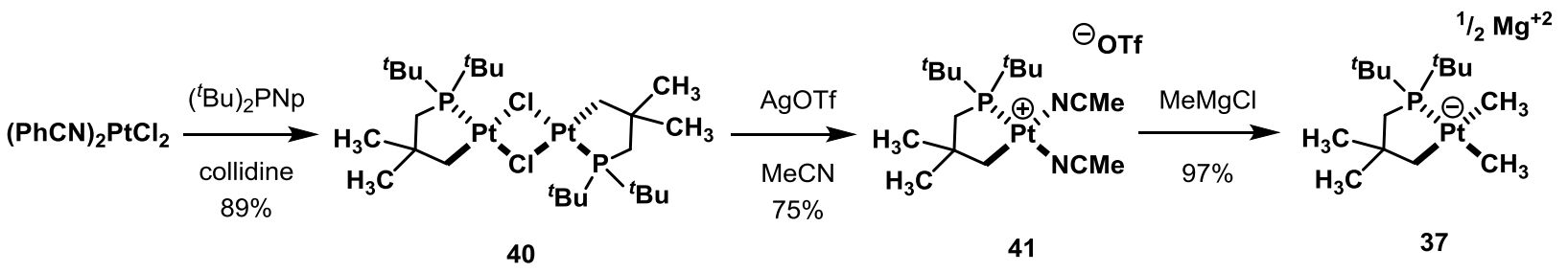

Scheme 23. Synthesis of 5-Membered Platinacycle Precatalysts

Platinates 37 through 39 were tested as precatalysts for hydrosilylation (Table 11). Although the conditions used in each reaction are not identical, none of the precatalysts perform better than precatalyst 16. Evidence from in situ ${ }^{31} \mathrm{P}$ NMR observation of reactions using precatalyst 37 suggests that the initial activation steps, which involve oxidative addition of triethoxysilane to $\mathrm{Pt}(\mathrm{II})$ anions, are slow and that some quantity of the precatalyst is trapped as a silylmethylplatinate during catalysis (Scheme 24). Because a four-coordinate square planar Pt(II) complex should undergo oxidative addition slowly, it is likely that for oxidative addition to platinates $\mathbf{3 2}$ and $\mathbf{3 7}$ the phosphine ligand must first dissociate. Because a 5-membered platinacycle does not contain as much angle $\operatorname{strain}^{32,33}$ as a 4-membered platinacycle, dissociation of the phosphine in the 5-membered platinacycle should be slower, and thus lead to slower oxidative addition, as we observe for platinate 37 . Deceleration of oxidative addition would also affect the rate of hydrosilylation using precatalyst 37. Although we are unable to distinguish the

${ }^{32}$ For seminal work on the concept of angle strain/ring strain, see: Von Baeyer, A. Ber. Dtsch. Chem. Ges. 1885, 18, 2278.

${ }^{33}$ For reviews on angle strain/ring strain, see: (a) Newton, M. D. Mod. Theor. Chem. 1977, 4, 223. (b) De Meijere, A. Angew. Chem. 1979, 91, 867. (c) Wiberg, K. B. Angew. Chem., Int. Ed. Engl. 1986, 25, 312. (d) Gimarc, B. M.; Zhao, M. Coord. Chem. Rev. 1997, 158, 385. 
effect of the 5-membered platinacycle on reaction rate from the effect of the platinacycle on catalyst activation, a reduced rate of hydrosilylation using $\mathbf{3 7}$ is consistent with phosphine dissociation as a step on the catalytic cycle prior to the turnover limiting step, as we have proposed in Scheme 22.

Table 11. Comparison of 5-Membered Platinacycle Precatalysts

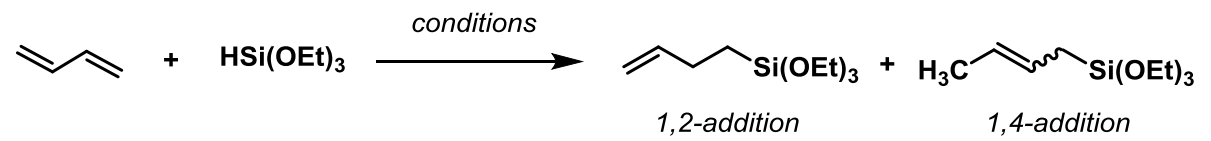

\begin{tabular}{|c|c|c|c|}
\hline Precatalyst & Reaction Conditions & Hydrosilylation Yield & 1,2-:1,4-Addition \\
\hline \multicolumn{4}{|c|}{$1 / 2 \mathrm{Mg}^{+2}$} \\
\hline \multirow[b]{4}{*}{37} & $0.5 \mathrm{~mol} \% 37$ & \multirow{4}{*}{$28 \%$} & \multirow{4}{*}{$9: 1$} \\
\hline & $2.0 \mathrm{~mol} \% \mathrm{MgCl}_{2}(\mathrm{THF})_{2}$ & & \\
\hline & $10 \mu \mathrm{L}$ THF & & \\
\hline & $-45^{\circ} \mathrm{C} \rightarrow 50{ }^{\circ} \mathrm{C} 25 \mathrm{~min}$ & & \\
\hline
\end{tabular}

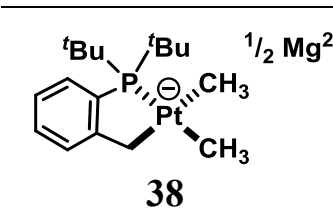

$$
\begin{gathered}
0.5 \mathrm{~mol} \% 38^{\circ} 38 \mathrm{MgCl}_{2}(\mathrm{THF})_{2} \\
0.5 \mathrm{~mol} \mathrm{\%} 10 \mathrm{THF} \\
0.5 \mathrm{~mL} \mathrm{DCM} \\
-45^{\circ} \mathrm{C} \rightarrow 50{ }^{\circ} \mathrm{C}, 25 \mathrm{~min} .
\end{gathered}
$$

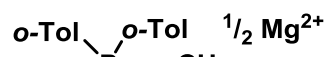

39

\section{$1.0 \mathrm{~mol} \% 39$}

$0.5 \mathrm{~mL} \mathrm{DCM}$ $-45^{\circ} \mathrm{C} \rightarrow 50{ }^{\circ} \mathrm{C}, 30 \mathrm{~min}$.

\section{$11 \%$}

$4: 1$

$$
16 \%
$$

${ }^{a}$ Yield and selectivity determined by integration of ${ }^{1} \mathrm{H}$ NMR resonances corresponding to the alkenyl hydrogen atoms vs. $\mathrm{TMS}_{2} \mathrm{O}$ (internal standard). ${ }^{b}$ Yield corresponds to conversion as no other products are observed. ${ }^{c}$ Isolated yield; reaction halted by $10 \mu \mathrm{L}$ N,N'-DMEDA after 25 min. 


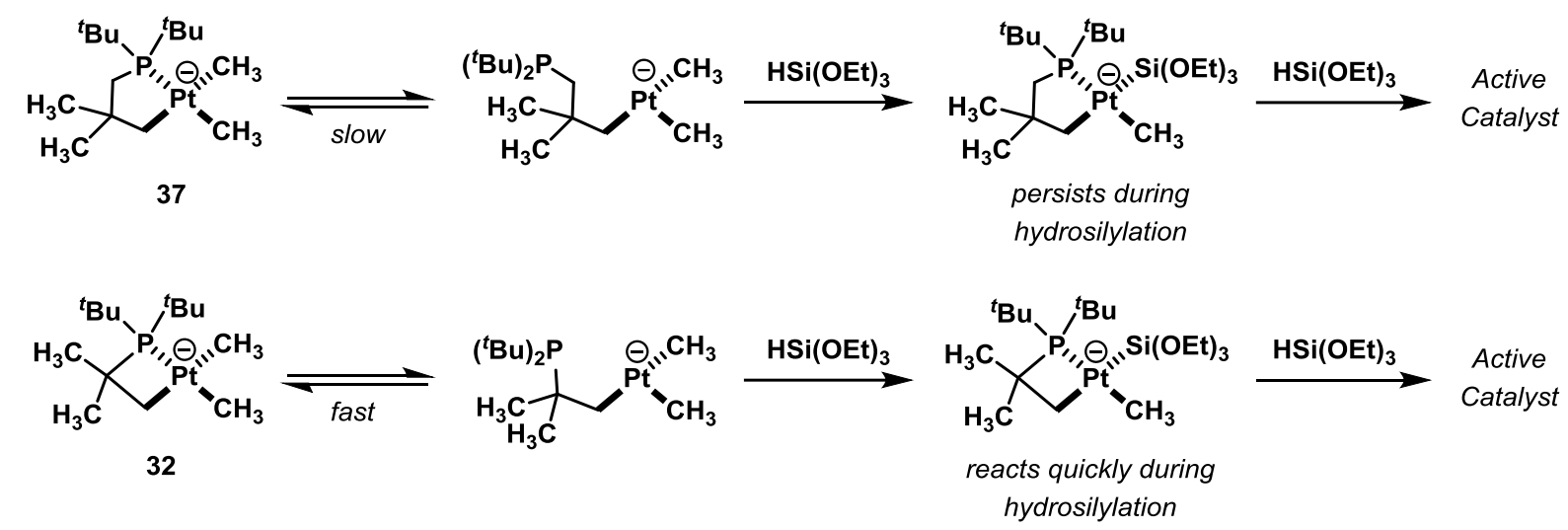

Scheme 24. Effect of 5-Membered Platinacycle on Catalyst Activation

\subsubsection{Evidence Against a Chalk-Harrod-Like Pt(0)/Pt(II) Catalytic Cycle}

Platinum catalyzed hydrosilylation reactions typically proceed by the Chalk-Harrod mechanism, which typically involves a $\mathrm{Pt}(0) / \mathrm{Pt}(\mathrm{II})$ redox couple (see section 1.2 .3$) .{ }^{34} \mathrm{The} \mathrm{Pt}(\mathrm{II}) / \mathrm{Pt}(\mathrm{IV})$ catalytic cycle we have proposed for the 1,2-selective hydrosilylation of dienes using precatalysts $\mathbf{1 6}, \mathbf{1 9}$, and 32 is not common for platinum-catalyzed hydrosilylation reactions. Given the near ubiquity of $\operatorname{Pt}(0) / \operatorname{Pt}(\mathrm{II})$ catalytic cycles in homogeneous hydrosilylation processes, we carefully considered the possibility of a ChalkHarrod-like $\mathrm{Pt}(0) / \mathrm{Pt}(\mathrm{II})$ catalytic cycle for hydrosilylation using precatalysts 16, 19, and 32.

A Chalk-Harrod-like $\mathrm{Pt}(0) / \mathrm{Pt}(\mathrm{II})$ catalytic cycle starting from precatalysts $\mathbf{1 6}, \mathbf{1 9}$, and $\mathbf{3 2}$ is shown in Scheme 25. Presumably, catalyst activation would proceed by reductive elimination of methyltriethoxysilane from precatalyst 19 to afford an electron-rich $\operatorname{Pt}(0)$ anion. Oxidative addition of triethoxysilane affords 5-coordinate Pt(II) complex B'. Platinum (II) shows a strong energetic preference for a square planar geometry, so butadiene may reversibly dissociate from intermediate B'. Intermediate B' is unlikely, however, to coordinate butadiene in an $\eta^{4}$-fashion, because $\eta^{4}$-coordination would generate

${ }^{34}$ For detailed theoretical discussion of $\mathrm{Pt}(0) / \mathrm{Pt}(\mathrm{II})$ catalyzed hydrosilylation, see: (a) Sakaki, S.; Ogawa, M.; Musashi, Y.; Arai, T. J. Am. Chem. Soc. 1994, 116, 7258. (b) Ozawa, F.; Hikida, T.; Hayashi, T. J. Am. Chem. Soc. 1994, 116, 2844. (c) Sakaki, S.; Mizoe, N.; Sugimoto, M. Organometallics 1998, 17, 2510. (d) Sakaki, S.; Mizoe, N.; Musashi, Y.; Biswas, B.; Sugimoto, M. J. Phys. Chem. A 1998, 102, 8027. (e) Sakaki, S.; Mizoe, N.; Musashi, Y.; Sugimoto, M. J. Mol. Struct.: THEOCHEM 1999, 461-462, 533. (f) Sakaki, S.; Mizoe, N.; Sugimoto, M.; Musashi, Y. Coord. Chem. Rev. 1999, 190-192, 933. (g) Giorgi, G.; De Angelis, F.; Re, N.; Sgamellotti, A. J. Mol. Struct.: THEOCHEM 2003, 623, 277. 
a formally $20 e^{-}$complex and require rearrangement to an energetically unfavorable octahedral geometry. Migratory insertion from intermediate B' is most likely to proceed with 1,2-selectivity to form intermediate C' because only one olefin of the diene is coordinated to the platinum center in B'. However, were 1,4-insertion to take place, a 5-coordinate complex would result which is energetically unfavorable for $d^{8}$ transition metals such as $\mathrm{Pt}(\mathrm{II})$. Intermediate $\mathbf{C}^{\prime}$ would then undergo reductive elimination to form the product alkenylsilane and regenerate $\mathbf{A}^{\prime}$.

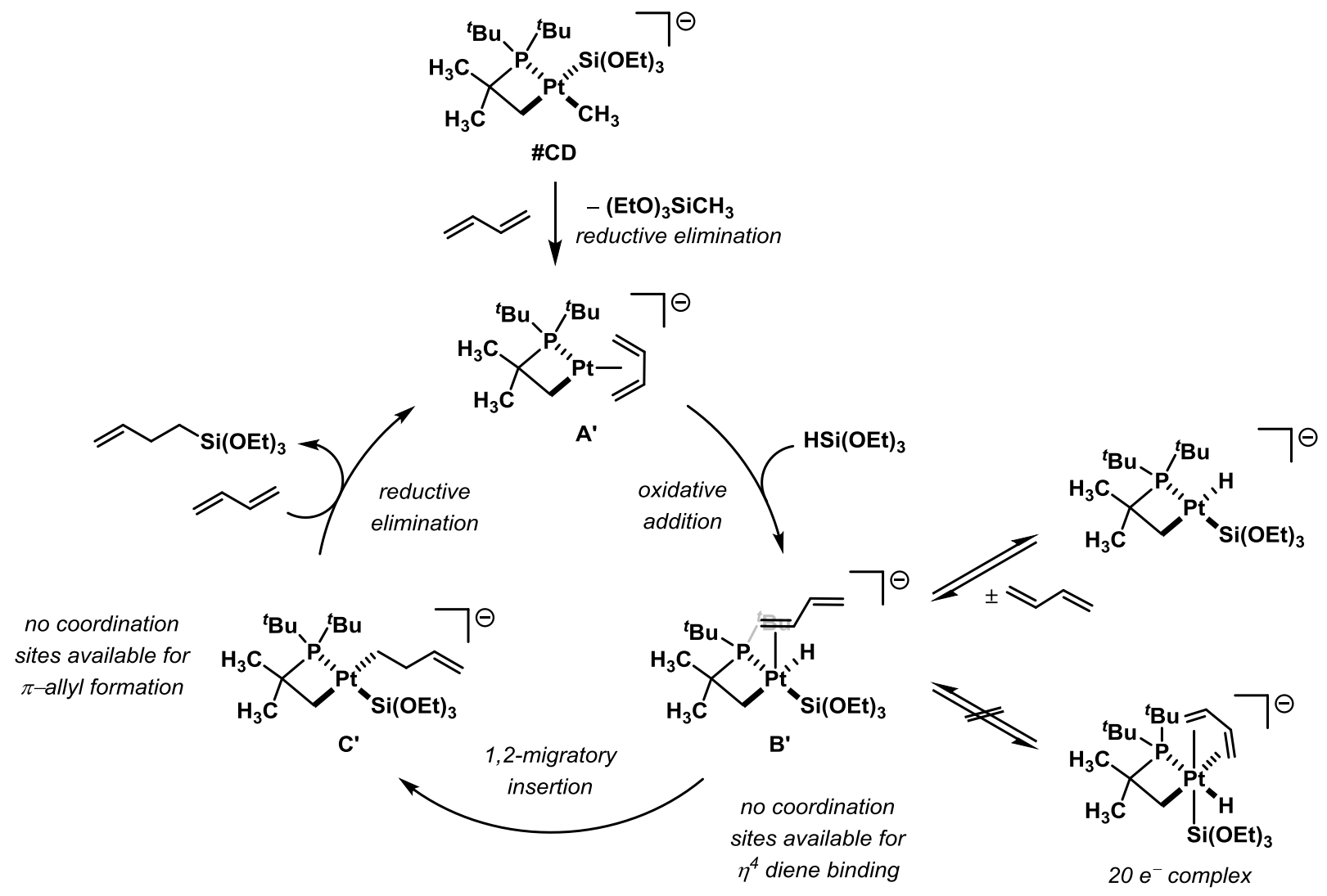

\section{Scheme 25. Chalk-Harrod-Like Pt(0)/Pt(II) Mechanism for Hydrosilylation of Butadiene}

The mechanism shown in Scheme 25 is plausible because it would account for the observed selectivity for 1,2-addition using precatalysts 16, 19, and 32. Intermediate $\mathbf{C}$ ', the product of 1,2migratory insertion of butadiene, is a $d^{8}$, square planar complex which should be energetically favored over the 5-coordinate complex that would result from 1,4-migratory insertion. Density functional theory 
calculations predict the difference in ground-state stability to be approximately $5 \mathrm{kcal} / \mathrm{mol}$, favoring the product of 1,2-insertion (Figure 7).
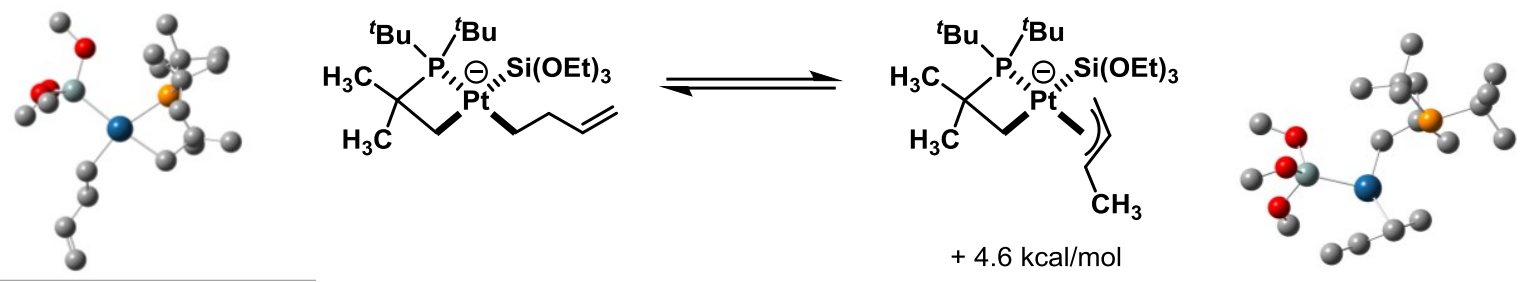

Figure 7. Optimized structures and calculated ground-state energies for the products of 1,2- vs. 1,4migratory insertion. Calculations performed at the B3PW91 level of theory, using a basis set that includes SDD quasirelativistic pseudopotentials on Pt (MWB60) extended by polarization functions (Pt: $\mathrm{f}, 0.993$ ), and $6-31 \mathrm{G}(\mathrm{d}, \mathrm{p})$ on $\mathrm{H}, \mathrm{C}, \mathrm{O}, \mathrm{Si}, \mathrm{P}$.

To test for a Chalk-Harrod-like Pt(0)/Pt(II) mechanism for 1,2-selective hydrosilylation using 16 and its derivatives, we attempted to synthesize several proposed intermediates on this catalytic cycle (Scheme 25) including A' and 'D' (complex 36). Upon two-electron reduction, precatalysts 16, 19, and 32 would bear a negative charge on the platinum center because the cyclometallated phosphine ligand includes a non-dissociable X-type ligand. Stable platinum(0) complexes typically bear electronwithdrawing or $\pi$-acceptor ligands, even when neutral, and the cyclometallated phosphine ligand is instead a strong $\sigma$-donor. To our knowledge, only a single anionic $\operatorname{Pt}(0)$ complexed by $\sigma$-donating phosphine or carbon ligands has been reported. ${ }^{35}$ This complex bears a tridentate ligand and can only be accessed with strong reducing agents such as $\mathrm{Na}^{0}$. Although we attempted to synthesize butadiene or COD-stabilized $\operatorname{Pt}(0)$ anions from 16 and related precursors, we were not able to observe a $\mathrm{Pt}(0)$ complex.

Synthesis of platinate D' was attempted via a dialkylplatinate intermediate similar to 32, but this route was unsuccessful because the alkenyl ligands underwent hydrosilylation faster than the platinate underwent oxidative addition (Scheme 26). Instead, we synthesized 36 from a neutral precursor bearing coordinated $\mathrm{MeCN}$, an easily displaced L-type ligand (Scheme 27). As a ligand, $\mathrm{MeCN}$ is not a strong $\sigma$ donor or a strong $\pi$-acceptor and it dissociates readily from $\mathrm{Pt}(\mathrm{II})$. Neutral complexes bearing $\mathrm{MeCN}$

${ }^{35}$ Schwartsburd, L.; Cohen, R.; Konstantinovski, L.; Milstein, D. Angew. Chem. Int. Ed. 2008, 47, 3603. 
ligands were prone to decomposition in solvents other than acetonitrile, as well as when subjected to vacuum for prolonged periods. Complex $\mathbf{3 6}$ was not stable to isolation and was formed in situ for testing as a hydrosilylation catalyst. We propose that $\mathbf{3 6}$ is unstable to isolation, unlike $\mathbf{1 9}$ and $\mathbf{3 2}$, because no additional equivalents of $\mathrm{MgCl}_{2}$ (THF) $)_{2}$ are present during the synthesis. Likewise, when 19 is synthesized from intermediate $\mathbf{4 2}$ and one equivalent of methylmagnesium chloride, 19 also exhibits partial decomposition upon isolation.

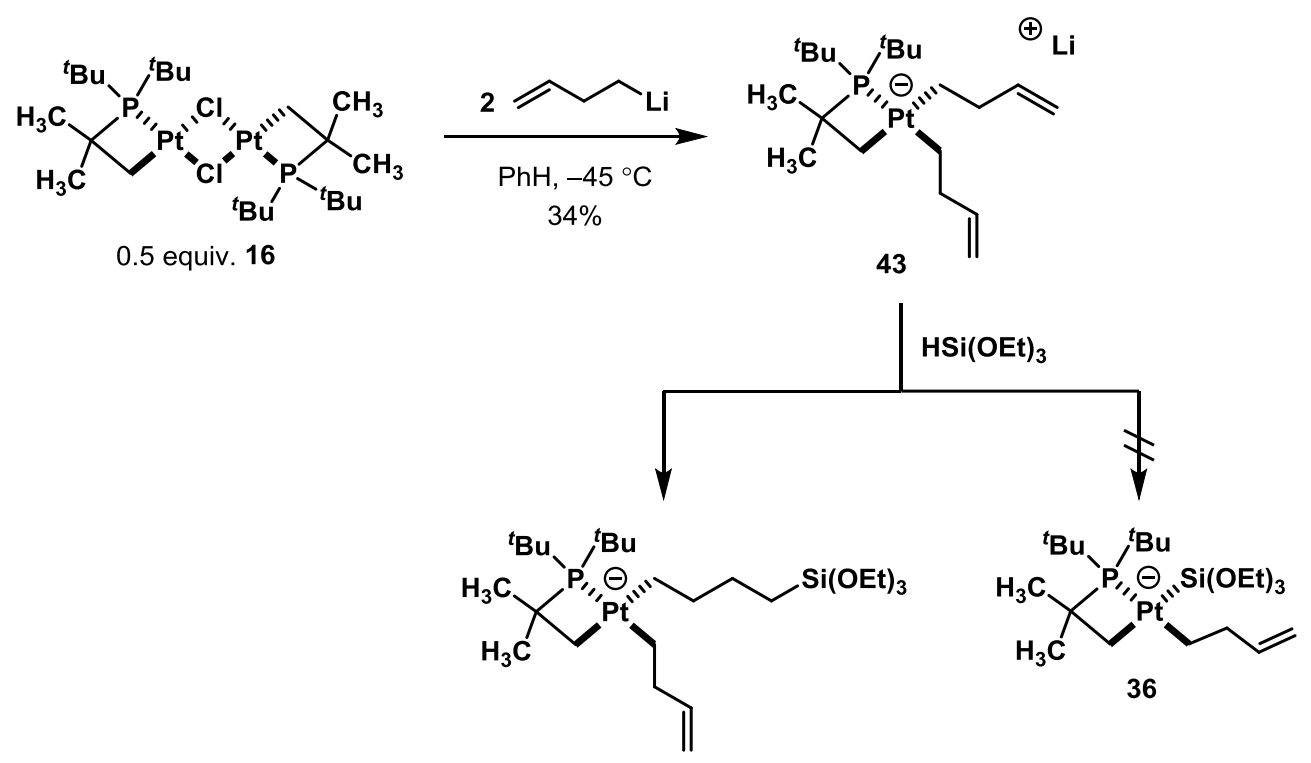

Scheme 26. Attempted Synthesis of 36 via Dialkenylplatinate Intermediate 43 


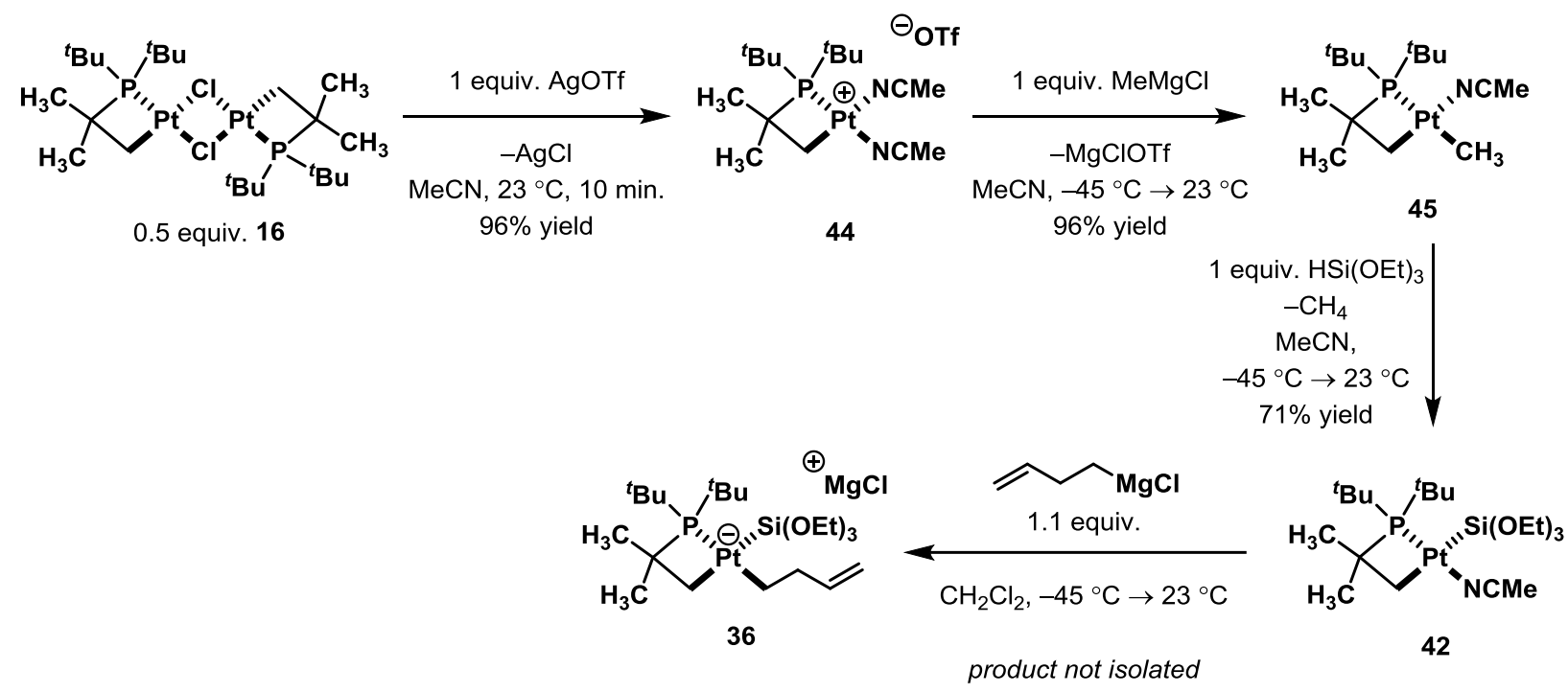

Scheme 27. Synthesis of Platinum Complex 36

Platinum complex 36 did not show any improvement in yield or selectivity over precatalysts $\mathbf{1 6}$, 19, and 32 in the hydrosilylation of butadiene, and complex $\mathbf{3 6}$ decomposed to tri-tert-butylphosphine during hydrosilylation. Because precatalysts 16, 19, and 32 show significant catalyst decomposition during activation, adding an equivalent amount of precatalyst $\mathbf{3 6}$ should result in a much larger proportion of active catalyst if $\mathbf{3 6}$ is an intermediate on the catalytic cycle. Because $\mathbf{3 6}$ showed no improvement in yield or selectivity over precatalysts $\mathbf{1 6}, \mathbf{1 9}$, and $\mathbf{3 2}$, complex 36 cannot be an intermediate on the catalytic cycle (Table 12). 
Table 12. Performance of Precatalyst 36 Compared to 16, 19, and 32

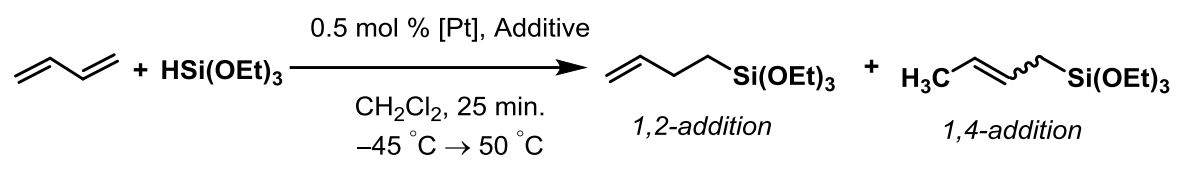

\begin{tabular}{clll} 
Precatalyst & Additive & Yield $^{a, b}$ & $\mathbf{1 , 2 : 1 ,}$ \\
\hline & & \\
\end{tabular}

16

standard conditions

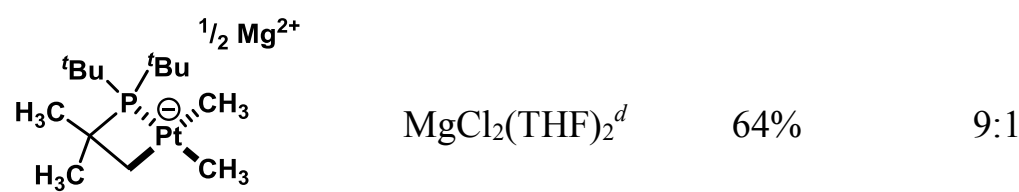

32

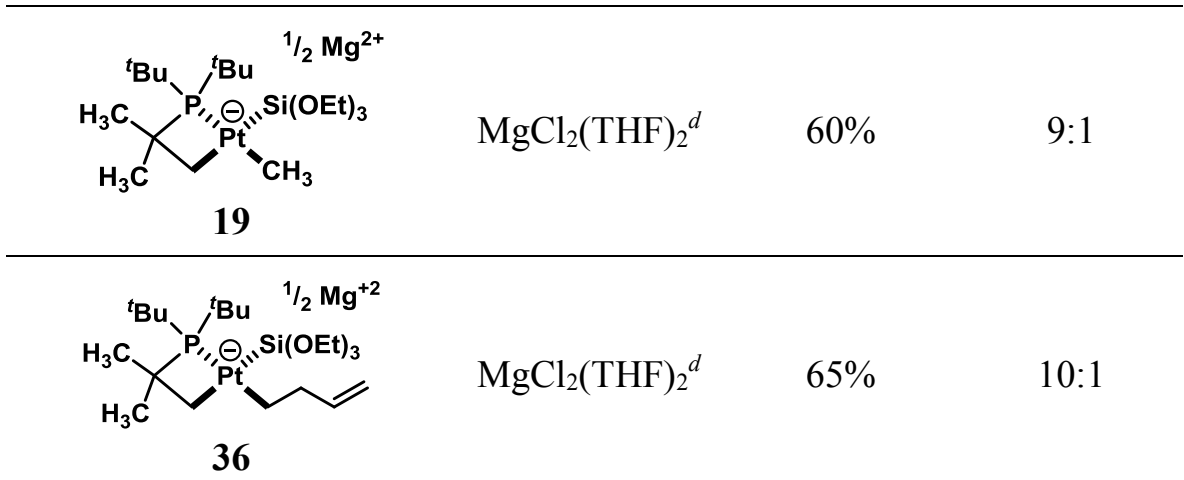

${ }^{a}$ Yield and selectivity determined by integration of ${ }^{1} \mathrm{H}$ NMR resonances corresponding to the alkenyl hydrogen atoms vs. $\mathrm{TMS}_{2} \mathrm{O}$ (internal standard). ${ }^{b}$ Yield corresponds to conversion as no other products are observed. ${ }^{d}$ Addition of $\mathrm{MgCl}_{2}(\mathrm{THF})_{2}$ mimics standard conditions for preactivated precatalysts.

Platinum complex 36 also did not undergo reductive elimination of butenylsilane when heated to $50{ }^{\circ} \mathrm{C}$ in the presence of butadiene for 5 minutes. Because $\sim 30$ catalyst turnovers occur during this time during the hydrosilylation of butadiene using precatalyst $\mathbf{1 6}$, the inability of $\mathbf{3 6}$ to undergo reductive elimination even once in this time kinetically excludes reductive elimination from $\mathbf{3 6}\left(\mathbf{C}^{\prime} \rightarrow \mathbf{A}^{\prime}\right)$ as a step on the catalytic cycle (Eq. 55). 


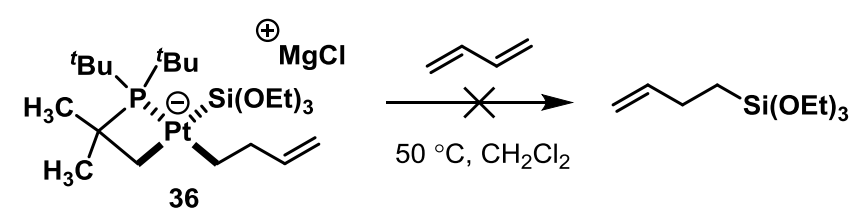

(Eq. 55)

\subsubsection{Alternate Mechanism Proposals}

Mechanism investigation requires the careful consideration and evaluation of many potential pathways because a mechanism can never be proven, only supported, by experimental data. Other mechanisms for the transformation reported herein have been proposed, the most relevant of which are $\mathrm{Pt}(0) / \mathrm{Pt}(\mathrm{II})$ catalysis from neutral platinum complexes and heterogeneous catalysis by platinum nanoparticles or colloids. In this section we present data for the 1,2-hydrosilylation that are not consistent with either of these mechanisms.

Precatalyst degradation could produce a neutral monodentate phosphine complexe of $\operatorname{Pt}(0)$ as the active catalyst (Eq. 56).

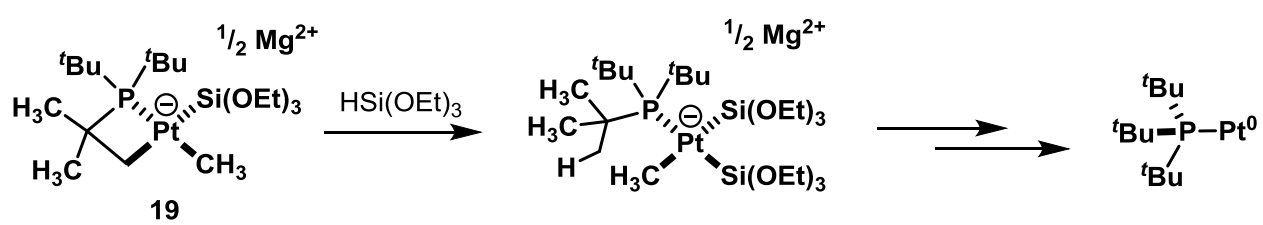

The formation of tri-tert-butylphosphine as a catalyst degradation product suggests that cyclometallation is lost during catalyst activation or degradation using precatalysts $\mathbf{1 6}, \mathbf{1 9}$, and $\mathbf{3 2}$, and we are unable to determine which from direct observation of platinum species during the reaction. To determine whether reduction of $\mathrm{Pt}(\mathrm{II})$ to $\mathrm{Pt}(0)$ and/or loss of cyclometallation is the critical step in catalyst activation, we tested a $\operatorname{Pt}(0)$ precursor with and without tri-tert-butylphosphine as a precatalyst for the hydrosilylation of butadiene. Karstedt's Catalyst ${ }^{36}$ is a commonly-used $\mathrm{Pt}(0)$ olefin hydrosilylation catalyst, and is known to allow for ready complexation with added ligands. Karstedt's Catalyst was not selective for 1,2-addition,

${ }^{36}$ (a) Karstedt, B. D. Ger. Offen. D.E. 2307085 A1, 1973. (b) Hitchcock, P. B.; Lappert, M. F.; Warhurst, N. J. W. Angew. Chem. 1991, 103, 439. 
alone or in the presence of $\mathrm{P}\left({ }^{t} \mathrm{Bu}\right)_{3}$. If formation of $\mathrm{Pt}(0)$ or loss of cyclometallation were the critical event in catalyst activation, we would expect to see selectivity for 1,2-addition from a $\operatorname{Pt}(0)$ phosphine catalyst generated by another method.

Table 13. Investigation of Pt(0) Precatalysts for Hydrosilylation of Butadiene

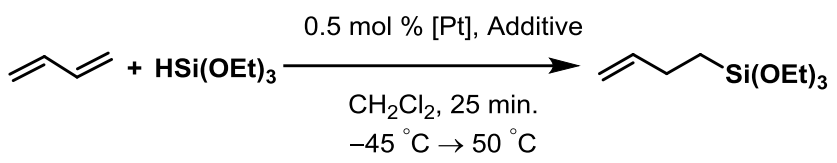

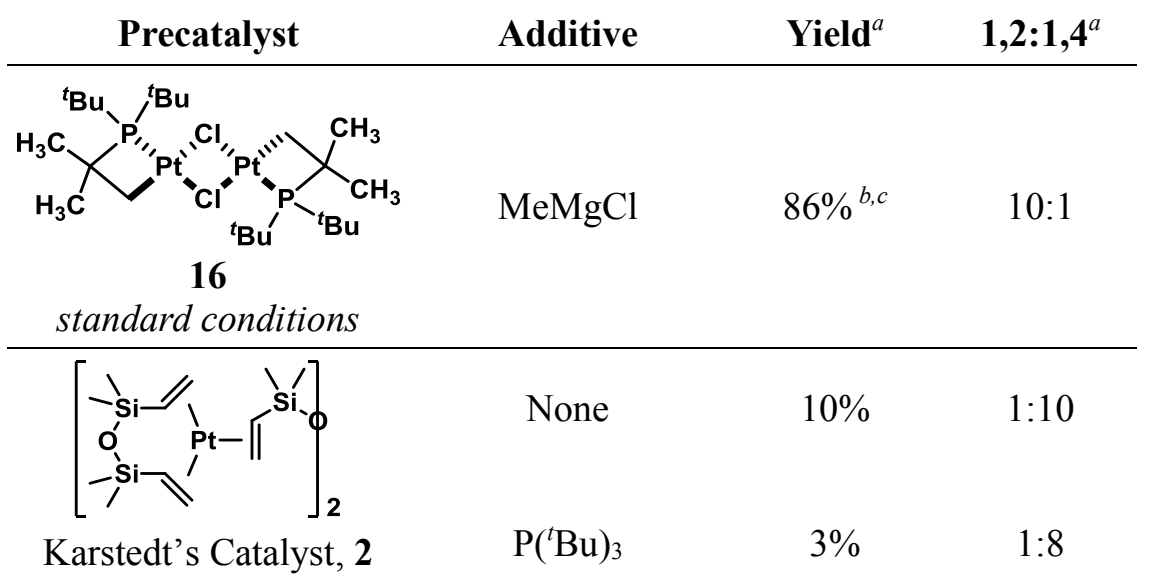

${ }^{a}$ Yield and selectivity determined by integration of ${ }^{1} \mathrm{H}$ NMR resonances corresponding to the alkenyl hydrogen atoms vs. $\mathrm{TMS}_{2} \mathrm{O}$ (internal standard). ${ }^{b}$ Yield corresponds to conversion as no other products are observed. ${ }^{c}$ Isolated yield; reaction halted by $10 \mu \mathrm{L} \mathrm{N}, \mathrm{N}^{\prime}$-DMEDA after $25 \mathrm{~min}$.

Degradation of homogeneous precatalysts can generate catalytically active metal nanoparticles and this mechanism must be carefully evaluated in reactions where catalyst degradation is observed. ${ }^{37,38}$ Addition of elemental mercury often disrupts catalysis at nanoparticle surfaces because mercury dissolves many metals, such as platinum, to form stable alloys. When nanoparticles are dissolved in metallic

${ }^{37}$ For review of colloidal or nanoparticulate metal catalysts, see: (a) Lewis, L. N. Chem. Rev. 1993, 93, 2693. (b) Johnson, B. F. G. Top. Catal. 2003, 24, 147. (c) Duran Pachon, L.; Rothenberg, G. Appl. Organomet. Chem. 2008, 22, 288. (d) Cheong, S.; Watt, J. D.; Tilley, R. D. Nanoscale 2010, 2, 2045. (e) Cong, H.; Porco, J. A. ACS Catal. 2012, 2, 65. (f) Yan, N.; Yuan, Y.; Dyson, P. J. Dalton Trans. 2013, 42, 13294.

${ }^{38}$ For examples of Pt nanoparticles as catalysts for hydrosilylation, see: (a) Lewis, L. N.; Uriarte, R. J. Organometallics 1990, 9, 621. (b) Lewis, L. N.; Uriarte, R. J.; Lewis, N. J. Mol. Catal. 1991, 66, 105. 
mercury, the nanoparticles are effectively isolated from the solution and cannot act as catalysts. ${ }^{39}$ In the case of hydrosilylation using precatalyst 19, addition of mercury showed only a mild effect on the observed selectivity and conversion. We conclude that catalysis by nanoparticulate platinum is unlikely to be the major pathway for hydrosilylation. However, because all precatalysts showed decomposition during activation, we were unable to obtain reproducible kinetic data to determine a rate law and we do not speculate as to the turnover-limiting step or the reversibility of proposed steps on the catalytic cycle.

\section{Table 14. Effect of $\mathbf{H g}^{0}$ on Hydrosilylation Using Precatalyst 19}

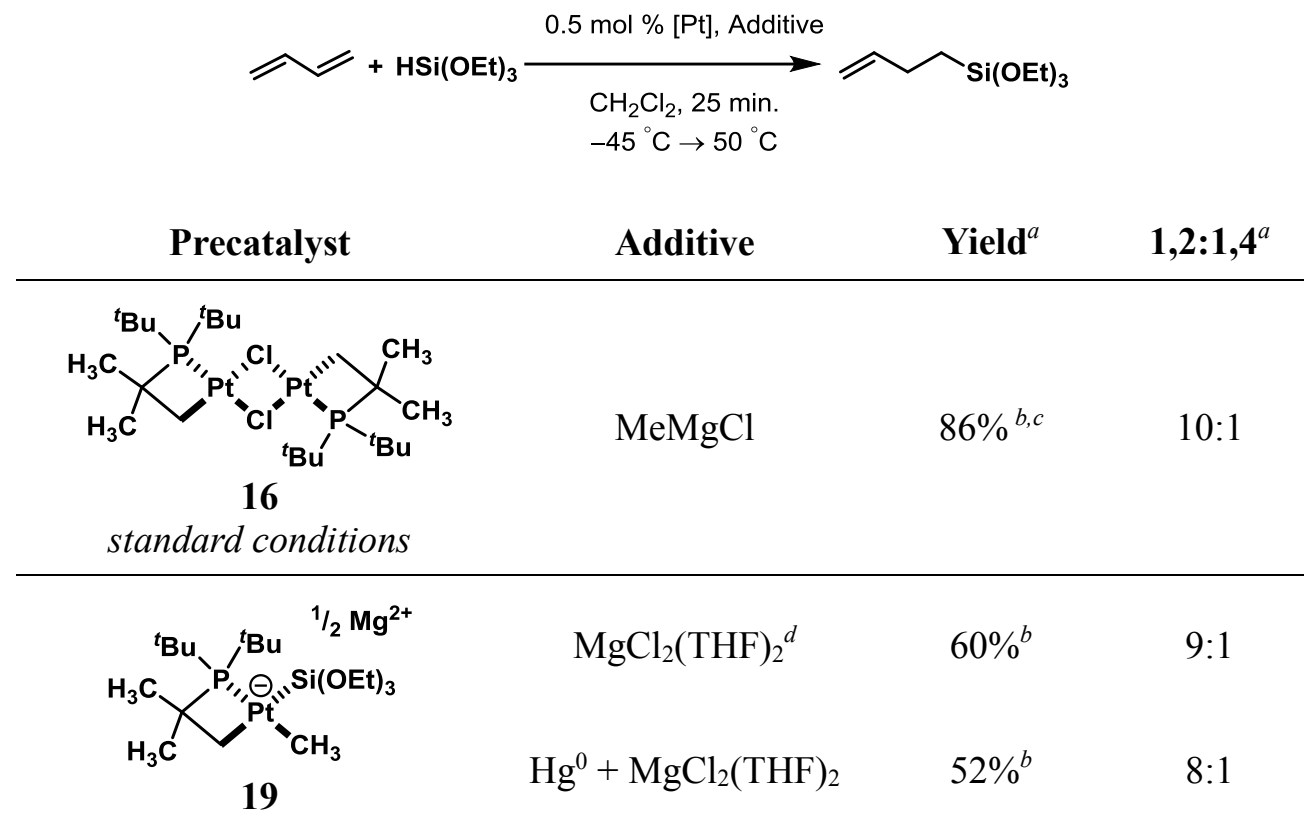

${ }^{a}$ Yield and selectivity determined by integration of ${ }^{1} \mathrm{H}$ NMR resonances corresponding to the alkenyl hydrogen atoms vs. TMS ${ }_{2} \mathrm{O}$ (internal standard). ${ }^{b}$ Yield corresponds to conversion as no other products are observed. ${ }^{c}$ Isolated yield; reaction halted by $10 \mu \mathrm{L}$ N,N'-DMEDA after $25 \mathrm{~min} .{ }^{d}$ Addition of $\mathrm{MgCl}_{2}(\mathrm{THF})_{2}$ simulates conditions for catalysis with precatalyst 16.

Other mechanism possibilities exist, including variations on both $\mathrm{Pt}(0 / \mathrm{II})$ and $\mathrm{Pt}(\mathrm{II} / \mathrm{IV})$ cycles. However, none that we have considered explain the unprecedented selectivity for 1,2-hydrosilylation that we observe using precatalysts $\mathbf{1 6}, \mathbf{1 9}$, and $\mathbf{3 2}$. Specifically, none is expected to prevent the formation of $\pi$ -

${ }^{39}$ Whitesides, G. M.; Hackett, M.; Brainard, R. L.; Lavalleye, J. P. P. M.; Sowinski, A. F.; Izumi, A. N.; Moore, S. S.; Brown, D. W.; Staudt, E. M. Organometallics 1985, 4, 1819. 
allyl intermediates. We propose that the platinacycle, which contributes both a non-dissociable ligand and a negative charge to the complex, is a key component that induces coordinative saturation and prevents $\pi$ allyl formation. In addition, the high electron density of platinates 16, 19, and 32 allows access to the + IV oxidation state, which is not typical for platinum catalysts under the reducing conditions generated by large quantities of hydrosilane that are necessary for hydrosilylation. 


\section{CONCLUSIONS AND OUTLOOK}

This Ph.D. thesis has described the discovery and development of the first general 1,2-selective hydrosilylation of butadiene and other 1,3-dienes. Selectivity for 1,2-addition results from coordinative saturation at catalyst intermediates that prevents the formation of $\pi$-allyl complexes. Although the current best catalyst activity and selectivity are insufficient for application in commercial organosilane production, future development could enable the synthesis of butenylsilane coupling agents for a variety of materials applications.

The biggest challenge remaining is one of catalyst stability and selectivity. Degradation of homogeneous precatalysts during catalyst activation limits our understanding of the mechanism by which 1,2-selective addition is achieved and hinders the development of better catalysts. With a clearer understanding of the mechanism of 1,2-hydrosilylation, catalysts with higher selectivity for 1,2-addition and potentially higher TON and TOF could be developed. Future work on this system could focus on the development of a precatalyst that undergoes activation cleanly, without degradation.

The fundamental advance described in this work is the development of a transition metal catalyst that avoids the formation of $\pi$-allyl complexes during catalysis. The thermodynamic favorability of $\pi$-allyl complexes has a strong influence on selectivity in many catalytic cycles. Future development of the concepts investigated in this thesis could lead to catalysts of the lighter transition metals, such as palladium or nickel, that are able to exclude $\pi$-allyl complex formation and selectively catalyze challenging transformations such as 1,2-hydrosilylation of dienes, allyl electrophiles, and other difficult substrates.

There are a number of other processes for which $\pi$-allyl formation controls reaction selectivity or causes the formation of undesired side products. This thesis has highlighted the 1,4-selective hydrosilylation of dienes as an example, but there are a number of other transformations, some conducted 
on extremely large scale for commercial synthesis, which would benefit from the development of catalysts that avoid $\pi$-allyl complex formation. Future work could investigate the hydrosilylation of allyl chloride, allyl acrylate, or styrene as potentially impactful substrates. 


\section{EXPERIMENTAL}

\subsection{Materials and Methods}

Reactions were carried out under ambient atmosphere unless otherwise noted. All air-sensitive compounds and reactions were performed under an inert atmosphere of nitrogen using standard Schlenk and glovebox techniques. ${ }^{1}$ All glassware was stored in an oven or flame-dried prior to use. Anhydrous solvents were obtained either by filtration through drying columns ${ }^{2}$ on an $\mathrm{mBraun}$ system $\left(\mathrm{Et} \mathrm{H}_{2} \mathrm{O}, \mathrm{DCM}\right.$, Pentane, Toluene), by distillation from $\mathrm{CaH}(\mathrm{MeCN}, \mathrm{DCE})$ or $\mathrm{Na} /$ benzophenone (Benzene, THF), or dried over $4 \AA$ molecular seives $\left(\mathrm{PhCN}, \mathrm{TMS}_{2} \mathrm{O}\right)$. Purified compounds were further dried under high vacuum (0.01-0.05 Torr). Yields refer to purified and spectroscopically pure compounds unless otherwise stated. Thin layer chromatography (TLC) was performed using EMD TLC plates pre-coated with $250 \mu \mathrm{m}$ thickness silica gel $60 \mathrm{~F}_{254}$ plates and visualized by fluorescence quenching under UV light and $\mathrm{KMnO}_{4}$ stain. Flash chromatography was performed using silica gel (230-400 mesh) purchased from Silicycle Inc.

NMR spectra were recorded on either a Varian Unity/Inova 600 spectrometer operating at 600 $\mathrm{MHz}$ for ${ }^{1} \mathrm{H}$ acquisitions, a Varian Unity/Inova 500 spectrometer operating at $500 \mathrm{MHz}$ and $125 \mathrm{MHz}$ for ${ }^{1} \mathrm{H}$ and ${ }^{13} \mathrm{C}$ acquisitions, respectively, or a Varian Mercury 400 spectrometer operating at $400 \mathrm{HMz}, 375$

$\mathrm{MHz}$, and $162 \mathrm{MHz}$ for ${ }^{1} \mathrm{H},{ }^{19} \mathrm{~F}$, and ${ }^{31} \mathrm{P}$ acquisitions, respectively. Chemical shifts for ${ }^{1} \mathrm{H}$ and ${ }^{13} \mathrm{C}$ acquisitions are reported in ppm with the solvent resonance as the internal standard $\left({ }^{1} \mathrm{H}: \mathrm{CDCl}_{3}, \delta 7.26\right.$; $\left(\mathrm{CD}_{3}\right)_{2} \mathrm{SO}, \delta 2.50 ; \mathrm{CD}_{3} \mathrm{CN}, \delta$ 1.94; $\left.\left(\mathrm{CD}_{3}\right)_{2} \mathrm{CO}, \delta 2.05\right),\left({ }^{13} \mathrm{C}: \mathrm{CDCl}_{3}, \delta 77.16 ; \mathrm{CD}_{3} \mathrm{CN}, \delta 1.32,\left(\mathrm{CD}_{3}\right)_{2} \mathrm{SO}, \delta\right.$

\footnotetext{
${ }^{1}$ Shriver, D. F.; Drezdon, M. A.; John Wiley \& Sons: New York, 1986.

${ }^{2}$ Pangborn, A. B.; Giardello, M. A.; Grubbs, R. H.; Rosen, R. K.; Timmers, F. J. Organometallics 1996, 15,1518 .
} 
39.52; $\left.\left(\mathrm{CD}_{3}\right)_{2} \mathrm{CO}, \delta 29.84,206.26\right) .{ }^{3}$ Chemical shifts for ${ }^{31} \mathrm{P}$ acquisitions are reported in ppm with $\mathrm{H}_{3} \mathrm{PO}_{4}$ as the external standard $\left({ }^{31} \mathrm{P}: \mathrm{CDCl}_{3}, \delta 0\right)$. Chemical shifts for ${ }^{19} \mathrm{~F}$ acquisitions are reported in ppm with $\mathrm{CFCl}_{3}$ as the external standard $\left({ }^{19} \mathrm{~F}: \mathrm{CDCl}_{3}, \delta 0\right)$. Data are reported as follows: $\mathrm{s}=$ singlet, $\mathrm{br}=$ broad, $\mathrm{d}=$ doublet, $\mathrm{t}=$ triplet, $\mathrm{q}=$ quartet, quin $=$ quintet, $\mathrm{m}=$ multiplet; coupling constants in Hz; integration. All deuterated solvents were purchased from Cambridge Isotope Laboratories, dried over $4 \AA$ molecular sieves (MS), and degassed by the freeze-pump-thaw method prior to use.

High-resolution mass spectra were obtained at the Harvard University Mass Spectrometry Facilities. High-resolution mass spectra were obtained on a Bruker Maxis Impact q-TOF. Low-resolution mass spectra were obtained on a Shimadzu GCMS-QP2010S equipped with an SHRXI-5MS column (30 $\mathrm{m} \times 0.25 \mathrm{~mm}$ i.d. $\times 0.25 \mu \mathrm{m}$ film thickness) operated under the following heating program: initial temperature $50{ }^{\circ} \mathrm{C}$ followed by a ramp of $20^{\circ} \mathrm{C} / \mathrm{min}$ to a final temperature of $250{ }^{\circ} \mathrm{C}$ which was held for 5 min. GC analysis was performed on an Agilent 7890A GC equipped with an HP-5 cross-linked methyl silicone column $(30 \mathrm{~m} \times 0.32 \mathrm{~mm}$ i.d. $\times 0.25 \mu \mathrm{m}$ film thickness $)$ operating under the following heating programs. Program 1: initial temperature of $50{ }^{\circ} \mathrm{C}$ followed by a ramp of $20{ }^{\circ} \mathrm{C} / \mathrm{min}$ to a final temperature of $250{ }^{\circ} \mathrm{C}$ and held for $5 \mathrm{~min}$. Program 2: initial temperature of $50{ }^{\circ} \mathrm{C}$ followed by a ramp of $2.5^{\circ} \mathrm{C} / \mathrm{min}$ to $85{ }^{\circ} \mathrm{C}$ followed by a ramp of $20^{\circ} \mathrm{C} / \mathrm{min}$ to a final temperature of $250{ }^{\circ} \mathrm{C}$. Retention times are reported in minutes followed by the integration. The butenylsilanes described herein were purified by bulb-to-bulb distillation using a Büchi B-585 Kugelrohr.

All reagents were purchased from Sigma Aldrich or Strem Chemicals unless otherwise noted. Butadiene and isoprene were dried with dibutylmagnesium and distilled prior to use. Myrcene and norbornadiene were distilled, dried over $4 \AA \mathrm{MS}$, and degassed by the freeze-pump-thaw method prior to use. Triethoxysilane, triethylsilane, and dimethylphenylsilane were distilled and degassed by the freezepump-thaw method prior to use. Triethylamine was dried over $\mathrm{CaH}_{2}$, distilled, and degassed by sparging

${ }^{3}$ Fulmer, G. R.; Miller, A. J. M.; Sherden, N. H.; Gottlieb, H. E.; Nudelman, A.; Stoltz, B. M.; Bercaw, J. E.; Goldberg, K. I. Organometallics 2010, 29, 2176. 
with argon prior to use. Bis-benzonitrileplatinum (II) chloride, ${ }^{4}$ 4-chlorobut-1-ene ${ }^{5}$, 3-butenylmagnesium chloride in $\mathrm{THF},{ }^{6}$ benzylpotassium, ${ }^{7}$ and $\mathrm{MgCl}_{2}(\mathrm{THF})_{2}{ }^{8}$ were prepared according to previously reported methods. Other chemicals were used as received.

Safety note regarding triethoxysilane: Triethoxysilane has been reported to form flammable gases upon exposure to hydrosilylation catalysts. ${ }^{9}$ A recommended substitute for triethoxysilane is diethoxymethylsilane.

\subsection{Synthesis of Platinum Complexes}

$\left[\kappa^{2}-\left(\left({ }^{\mathrm{B} u}\right)_{2} \mathrm{PCMe}_{2} \mathrm{CH}_{2}\right) \mathrm{PtCl}_{2}\right.$

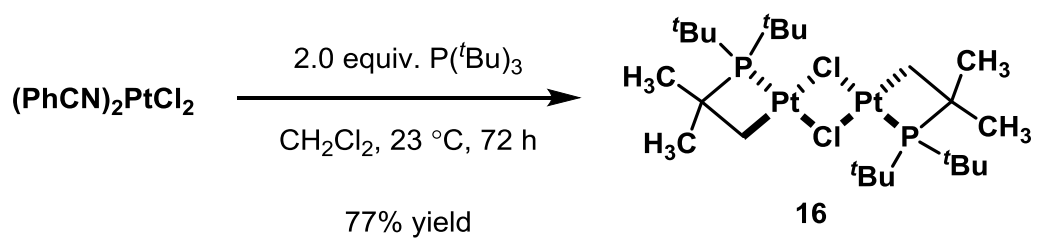

Platinum precatalyst $\mathbf{1 6}$ was prepared according to the method of Clark et al. ${ }^{10}$ In a dry, $\mathrm{N}_{2}$-filled glovebox, $\mathrm{Pt}(\mathrm{PhCN})_{2} \mathrm{Cl}_{2}$ (1.18 g, $2.49 \mathrm{mmol}, 1.00$ equiv.), a Teflon-coated magnetic stirring bar, and dichloromethane $(2.5 \mathrm{~mL})$ were added to a $20 \mathrm{~mL}$ scintillation vial at $23{ }^{\circ} \mathrm{C}$. While stirring, a solution of $\mathrm{P}\left({ }^{t} \mathrm{Bu}\right)_{3}$ (1.01 g, $4.98 \mathrm{mmol}, 2.00$ equiv.) in dichloromethane $(3.0 \mathrm{~mL})$ was added dropwise.

${ }^{4}$ (a) Braunstein, P.; Bender, R.; Jud, J. Inorg. Synth. 1989, 26, 341. (b) Kiyooka, S.-I.; Matsumoto, S.; Shibata, T.; Shinozaki, K.-I. Tetrahedron 2010, 66, 1806.

${ }^{5}$ Schmidt, T.; Kirschning, A. Angew. Chem., Int. Ed. 2012, 51, 1063.

${ }^{6}$ Trust, R. I.; Ireland, R. E. Org. Syn. 1973, 53, 116.

${ }^{7}$ Lochmann, L.; Trekoval, J. J. Organomet. Chem. 1987, 326, 1.

${ }^{8}$ Sivaram, S.; Satyanarayana, G. IN186303A1, 2001.

9 (a) Berk, S. C.; Buchwald, S. L. J. Org. Chem. 1992, 57, 3751. (b) Buchwald, S. L.; Gutierrez, A.; Berk, S. C.; Kreutzer, K. A. US5220020A, 1993. (c) Buchwald, S. L. Chem. Eng. News 1993, 71, 2.

${ }^{10}$ Clark, H. C.; Goel, A. B.; Goel, R. G.; Goel, S. Inorg. Chem. 1980, 19, 3220. 
Dichloromethane $(3 \times 1.5 \mathrm{~mL})$ was used to rinse the vial that had contained the phosphine solution and the rinsate was added to the reaction mixture. After $30 \mathrm{~min}$, the solids had dissolved to form a clear red solution that faded to pale yellow after 72 hours at $23{ }^{\circ} \mathrm{C}$. The solution was concentrated by rotary evaporation to $25 \%$ of its initial volume, then diluted with ethanol $(10 \mathrm{~mL})$ and stirred for $20 \mathrm{~min}$. at 23 ${ }^{\circ} \mathrm{C}$, at which time a colorless precipitate was observed. Centrifugation followed by decantation of the supernatant yielded a colorless solid that was washed with EtOH $(2 \times 10 \mathrm{~mL})$ and hexanes $(3 \times 10 \mathrm{~mL})$ to yield the title compound as a colorless solid $(0.832 \mathrm{~g}, 0.964 \mathrm{mmol}, 77 \%$ yield $)$. The product was used as obtained, but if desired, $\mathbf{1 6}$ can be recrystallized by cooling a saturated solution of $\mathbf{1 6}$ (toluene, $50{ }^{\circ} \mathrm{C}$ ) at $-35{ }^{\circ} \mathrm{C}$ for 24 hours to yield clear, colorless crystals.

NMR Spectroscopy: ${ }^{1} \mathrm{H}$ NMR (400 MHz, $\left.\mathrm{CD}_{2} \mathrm{Cl}_{2}, 25{ }^{\circ} \mathrm{C}, \delta\right): 1.56-1.41(\mathrm{~m}, 48 \mathrm{H}), 1.36\left(\mathrm{~d},{ }^{3} \mathrm{~J}_{\mathrm{P}-\mathrm{H}}=7.4 \mathrm{~Hz}\right.$, $2 \mathrm{H}), 1.30\left(\mathrm{~d},{ }^{3} J_{\mathrm{P}-\mathrm{H}}=7.4 \mathrm{~Hz}, 1.2 \mathrm{H}\right) .{ }^{13} \mathrm{C} \mathrm{NMR}\left(100 \mathrm{MHz}, \mathrm{CD}_{2} \mathrm{Cl}_{2}, 25{ }^{\circ} \mathrm{C}, \delta\right): 55.0\left(\mathrm{~d}, J_{\mathrm{P}-\mathrm{C}}=27.6 \mathrm{~Hz}\right)$, $38.4\left(\mathrm{~d}, J_{\mathrm{P}-\mathrm{C}}=17.5 \mathrm{~Hz}\right), 38.3\left(\mathrm{~d}, J_{\mathrm{P}-\mathrm{C}}=17.5 \mathrm{~Hz}\right), 32.5-30.5(\mathrm{~m}),-0.66\left(\mathrm{~d}, J_{\mathrm{P}-\mathrm{C}}=27.6 \mathrm{~Hz}\right),-0.85\left(\mathrm{~d}, J_{\mathrm{P}-\mathrm{C}}\right.$ $=27.6 \mathrm{~Hz}) .{ }^{31} \mathrm{P}$ NMR $\left(162 \mathrm{MHz}, \mathrm{CD}_{2} \mathrm{Cl}_{2}, 25{ }^{\circ} \mathrm{C}, \delta\right):-15.5\left({ }^{1} J_{\mathrm{P}-\mathrm{Pt}}=3761 \mathrm{~Hz}\right),-15.9\left({ }^{1} J_{\mathrm{P}-\mathrm{Pt}}=3747 \mathrm{~Hz}\right)$. Spectroscopic data match those reported for the title compound as a 2:1 mixture of head-to-tail and headto-head dimeric structures.

\section{$\kappa^{2}-\left(\left({ }^{\mathrm{B} u}\right)_{2} \mathrm{PCMe}_{2} \mathrm{CH}_{2}\right) \operatorname{PtCl}\left(\mathrm{P}\left({ }^{\mathrm{B} u}\right)_{3}\right)(17)$}

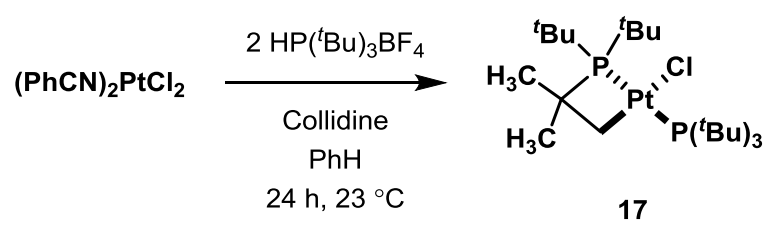

Preparation of compound $\mathbf{1 7}$ was adapted from the procedure of Clark and Goel. ${ }^{10}$ In a dry, $\mathrm{N}_{2}$-filled glovebox, $(\mathrm{PhCN})_{2} \mathrm{PtCl}_{2}$ (100. mg, $0.212 \mathrm{mmol}, 1.00$ equiv.), $\mathrm{HP}\left({ }^{t} \mathrm{Bu}\right)_{3} \mathrm{BF}_{4}$ (123 mg, $0.424 \mathrm{mmol}, 2.00$ equiv.), 2,4,6-trimethylpyridine ( $84 \mu \mathrm{L}, 77 \mathrm{mg}, 0.64 \mathrm{mmol}, 3.0$ equiv.), benzene ( $1 \mathrm{~mL}$ ), and a Tefloncoated magnetic stirring bar were added to a $4 \mathrm{~mL}$ glass scintillation vial. The clear yellow solution was stirred for 14 hours at $23{ }^{\circ} \mathrm{C}$, then concentrated under reduced pressure. The residue was extracted with 
hexanes $(2 \times 2 \mathrm{~mL})$ and the extracts filtered through glass wool and concentrated under reduced pressure to yield the title compound as a colorless solid.

NMR Spectroscopy: ${ }^{1} \mathrm{H}$ NMR $\left(400 \mathrm{MHz}, \mathrm{C}_{6} \mathrm{D}_{6}, 23{ }^{\circ} \mathrm{C}, \delta\right): 1.67\left(\mathrm{~d},{ }^{2} J_{\mathrm{P}-\mathrm{H}}=12.4 \mathrm{~Hz}, 27 \mathrm{H}\right), 1.54\left(\mathrm{~d},{ }^{2} J_{\mathrm{P}-\mathrm{H}}=\right.$ $12.8 \mathrm{~Hz}, 6 \mathrm{H}), 1.52\left(\mathrm{~d},{ }^{2} J_{\mathrm{P}-\mathrm{H}}=11.7 \mathrm{~Hz}, 18 \mathrm{H}\right), 1.27\left(\mathrm{dt},{ }^{2} J_{\mathrm{P}-\mathrm{H}}=17.2 \mathrm{~Hz},{ }^{2} J_{\mathrm{P}-\mathrm{H}}=2.6 \mathrm{~Hz}, 2 \mathrm{H}\right) .{ }^{31} \mathrm{P} \mathrm{NMR}$ $\left(162 \mathrm{MHz}, \mathrm{C}_{6} \mathrm{D}_{6}, 25{ }^{\circ} \mathrm{C}, \delta\right): 68.9\left({ }^{1} J_{\mathrm{P}-\mathrm{Pt}}=2696 \mathrm{~Hz},{ }^{2} J_{\mathrm{P}-\mathrm{P}}=383 \mathrm{~Hz}\right),-10.2\left({ }^{1} J_{\mathrm{P}-\mathrm{Pt}}=2359 \mathrm{~Hz},{ }^{1} J_{\mathrm{P}-\mathrm{Pt}}=383\right.$ $\mathrm{Hz})$.

\section{$\left[\kappa^{2}-\left(\left({ }^{t} \mathrm{Bu}\right)_{2} \mathrm{PCMe} \mathrm{CH}_{2}\right) \operatorname{Pt}\left(\mathrm{CH}_{3}\right)_{2}\right]\left[\mathrm{Mg}(\mathrm{THF})_{4}\right]_{\mathrm{n}}(32)$}

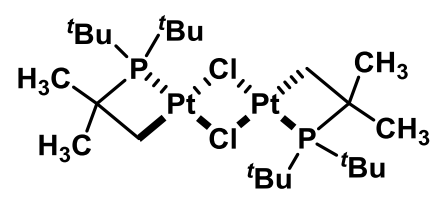

0.5 equiv. 16

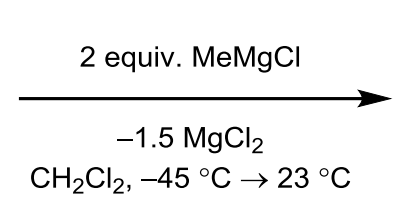

$90 \%$ yield

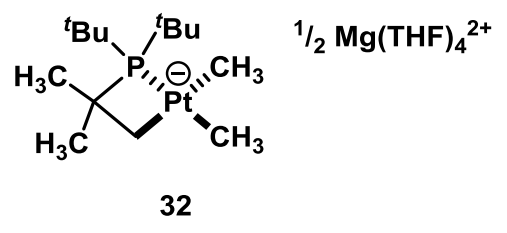

32

In a dry, $\mathrm{N}_{2}$-filled glovebox, Pt-chloride dimer $16(50.0 \mathrm{mg}, 58.0 \mu \mathrm{mol}, 1.00$ equiv.), dichloromethane $(1.5 \mathrm{~mL})$, and a Teflon-coated magnetic stirring bar were added to a $4 \mathrm{~mL}$ scintillation vial at $23{ }^{\circ} \mathrm{C}$. The vial was chilled at $-45^{\circ} \mathrm{C}$ in a $\mathrm{CO}_{2} / \mathrm{PrOH}$-cooled cold well for $30 \mathrm{~min}$. and methylmagnesium chloride in THF (c = 3.28 M, $71.3 \mu \mathrm{L}, 0.232 \mathrm{mmol}, 4.00$ equiv.) was added. The reaction vial was sealed with a Teflon-lined cap and the vial was removed from the cold well and stirred at $23{ }^{\circ} \mathrm{C}$ for 90 seconds to dissolve frozen droplets of Grignard reagent. The solution was stirred at $-45{ }^{\circ} \mathrm{C}$ for 3 hours, then at $23{ }^{\circ} \mathrm{C}$ for 30 min., at which time precipitation of a white solid was observed. Filtration through glass wool afforded a clear colorless solution which was diluted with benzene $(1.5 \mathrm{~mL})$ and concentrated in vacuo to half the orginial volume. The remaining solution was frozen at $-45{ }^{\circ} \mathrm{C}$ and the resulting solid was lyophilized. The title compound was isolated as a white powder $(73.3 \mathrm{mg})$ containing the product and aggregated $\mathrm{MgCl}_{2}(\mathrm{THF})_{2}$. The product could not be purified by recrystallization due to the high solubility of 32 in organic solvents and the high lattice energy of $\mathrm{MgCl}_{2}(\mathrm{THF})_{2}$. Attempts to purify 32 by crystallization yielded decomposition of $\mathbf{3 2}$ and crystalline magnesium salts. The tendency of $\mathbf{3 2}$ to 
aggregate with $\mathrm{MgCl}_{2}(\mathrm{THF})_{2}$ and its resistance to further purification by recrystallization prevented us from obtaining satisfactory results from elemental microanalysis. Reaction yield and purity were determined in a separate experiment from ${ }^{1} \mathrm{H}$ NMR integrations relative to an internal standard.

\section{Yield determination:}

In a dry, $\mathrm{N}_{2}$-filled glovebox, Pt-chloride dimer $16(25.4 \mathrm{mg}, 29.0 \mu \mathrm{mol}, 1.00$ equiv. $)$ and dichloromethane- $d_{2}(0.5 \mathrm{~mL})$ were added to a $4 \mathrm{~mL}$ scintillation vial to form a clear colorless solution. The vial was cooled at $-45{ }^{\circ} \mathrm{C}$ in a $\mathrm{CO}_{2} /{ }^{i} \mathrm{PrOH}-$ cooled cold well for $30 \mathrm{~min}$. and methylmagnesium chloride in THF ( $\mathrm{c}=3.33 \mathrm{M}, 35.7 \mu \mathrm{L}, 0.119 \mathrm{mmol}, 4.00$ equiv.) was added. The reaction vial was sealed with a Teflon-lined cap and the vial was shaken for 90 seconds at $23{ }^{\circ} \mathrm{C}$ to dissolve frozen droplets of the Grignard reagent. The reaction vial was cooled $-45{ }^{\circ} \mathrm{C}$ for 3 hours, then warmed at $23{ }^{\circ} \mathrm{C}$ for 30 min., at which time precipitation of a white solid was observed. The reaction vial was unsealed and 1,5cyclooctadiene (internal standard, $7.2 \mu \mathrm{L}, 6.4 \mathrm{mg}, 59 \mu \mathrm{mol}, 2.0$ equiv.) was added and the contents of the vial mixed thoroughly. The white mixture was transferred to an NMR tube which was sealed with a plastic cap and electrical tape. ${ }^{1} \mathrm{H}$ NMR analysis showed the title compound, 32, in $98 \%$ yield relative to the internal standard. ${ }^{31} \mathrm{P}$ NMR of this sample showed only the signal for the title compound.

NMR Spectroscopy: ${ }^{1} \mathrm{H}$ NMR (600 MHz, $\left.\mathrm{CD}_{2} \mathrm{Cl}_{2}, 25{ }^{\circ} \mathrm{C}, \delta\right): 4.08$ (s, br, 12H), 1.94 (s, br, 12H), 1.42 (d, $\left.{ }^{3} J_{\mathrm{P}-\mathrm{H}}=11.2 \mathrm{~Hz}, 24 \mathrm{H}\right), 0.60\left(\mathrm{~d},{ }^{3} J_{\mathrm{P}-\mathrm{H}}=13.5 \mathrm{~Hz},{ }^{2} J_{\mathrm{Pt}-\mathrm{H}}=61.0 \mathrm{~Hz}, 2 \mathrm{H}\right), 0.36\left(\mathrm{~d},{ }^{3} J_{\mathrm{P}-\mathrm{H}}=4.7 \mathrm{~Hz},{ }^{2} J_{\mathrm{Pt}-\mathrm{H}}=47.0\right.$ $\mathrm{Hz}, 3 \mathrm{H}), 0.16\left(\mathrm{~d},{ }^{3} J_{\mathrm{P}-\mathrm{H}}=2.9 \mathrm{~Hz},{ }^{2} J_{\mathrm{Pt}-\mathrm{H}}=30.5 \mathrm{~Hz}\right) .{ }^{13} \mathrm{C} \mathrm{NMR}\left(125 \mathrm{MHz}, \mathrm{CD}_{2} \mathrm{Cl}_{2}, 25{ }^{\circ} \mathrm{C}, \delta\right): 70.0,54.8(\mathrm{~d}$, $\left.J_{\mathrm{P}-\mathrm{C}}=25.3 \mathrm{~Hz}\right), 36.0,35.0\left(\mathrm{~s}, J_{\mathrm{Pt}-\mathrm{C}}=43.7 \mathrm{~Hz}\right), 32.0,25.5,20.1\left(\mathrm{~d}, J_{\mathrm{P}-\mathrm{C}}=19.9 \mathrm{~Hz}, J_{\mathrm{Pt}-\mathrm{C}}=452.4 \mathrm{~Hz}\right),-9.3$ $\left(\mathrm{d}, J_{\mathrm{P}-\mathrm{C}}=88.2 \mathrm{~Hz}\right),-17.5\left(\mathrm{~s}, J_{\mathrm{Pt}-\mathrm{C}}=279.9 \mathrm{~Hz}\right) .{ }^{31} \mathrm{P} \mathrm{NMR}\left(162 \mathrm{MHz}, \mathrm{CD}_{2} \mathrm{Cl}_{2}, 25{ }^{\circ} \mathrm{C}, \delta\right): 9.4\left({ }^{1} J_{\mathrm{P}-\mathrm{Pt}}=1814\right.$ $\mathrm{Hz})$. 


\section{$\left[\kappa^{2}-\left(\left({ }^{t} \mathrm{Bu}\right)_{2} \mathrm{PCMe}_{2} \mathrm{CH}_{2}\right) \operatorname{Pt}\left(\mathrm{CH}_{3}\right)\left(\mathrm{Si}(\mathrm{OEt})_{3}\right)\right]\left[\mathrm{Mg}(\mathrm{THF})_{4}\right]_{\mathrm{n}}(19)$}

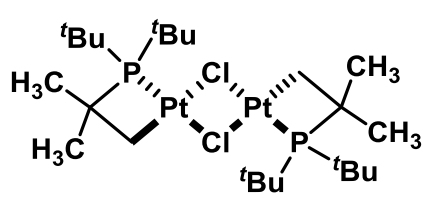

0.5 equiv. 16

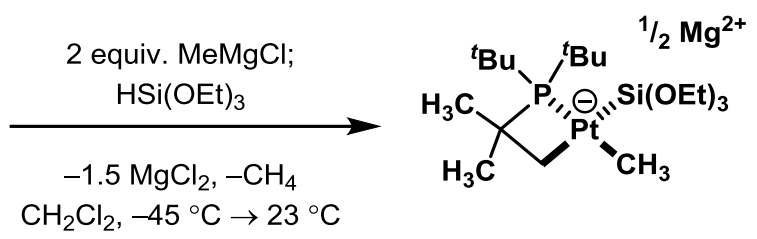

19

$96 \%$ yield

The title compound can be prepared from Pt-chloride dimer $\mathbf{1 6}$ or from dimethyl platinate $\mathbf{3 2}$. In this procedure, we describe in situ generation of dimethyl platinate $\mathbf{5}$ followed by transformation to silyl methyl platinate 19.

\section{Formation of platinate 32:}

In a dry, $\mathrm{N}_{2}$-filled glovebox, Pt-chloride dimer $16(50.0 \mathrm{mg}, 58.0 \mu \mathrm{mol}, 1.00$ equiv. $)$, dichloromethane $(1.5 \mathrm{~mL})$, and a Teflon-coated magnetic stirring bar were added to a $4 \mathrm{~mL}$ scintillation vial at $23{ }^{\circ} \mathrm{C}$. The vial was cooled at $-45{ }^{\circ} \mathrm{C}$ in a $\mathrm{CO}_{2} /{ }^{i} \mathrm{PrOH}-$ cooled cold well for $30 \mathrm{~min}$. Methylmagnesium chloride in THF (c = 3.28 M, 71.3 $\mu \mathrm{L}, 0.232 \mathrm{mmol}, 4.00$ equiv.) was added, the reaction vial was sealed with a Teflon-lined cap, and the vial was removed from the cold well and shaken at $23{ }^{\circ} \mathrm{C}$ for 90 seconds to dissolve frozen droplets of Grignard reagent. The solution was stirred at $-45^{\circ} \mathrm{C}$ for 3 hours, then at $23{ }^{\circ} \mathrm{C}$ for $30 \mathrm{~min}$., at which time precipitation of a white solid was observed.

\section{Silylation to form platinate 19:}

The reaction vial was chilled at $-45^{\circ} \mathrm{C}$ for 30 minutes, then butadiene $(100 \mu \mathrm{L})$ and triethoxysilane $(112$ $\mu \mathrm{L}, 99.7 \mathrm{mg}, 0.609 \mathrm{mmol}, 2.00$ equiv.) were added. The solution was stirred for $60 \mathrm{~min}$. at $23{ }^{\circ} \mathrm{C}$. Filtration through glass wool afforded a clear, pale yellow solution which was reduced in volume to $\sim 3$ $\mathrm{mL}$ in vacuo, diluted with benzene $(5 \mathrm{~mL})$, and frozen at $-45{ }^{\circ} \mathrm{C}$ before the resulting solid was lyophilized. The title compound was isolated as a pale yellow powder (499 mg). Attempts to purify 6 by recrystallization yielded mixtures of crystals containing crystals of 19 in multiple aggregation modes (see X-ray Crystallographic Analysis section) and crystalline magnesium salts. The tendency of $\mathbf{6}$ to aggregate 
with $\mathrm{MgCl}_{2}$ (THF) $)_{2}$ and its resistance to further purification by recrystallization prevented us from obtaining satisfactory results from elemental microanalysis. Reaction yield and purity was determined in a separate experiment from ${ }^{1} \mathrm{H}$ NMR integrations relative to an internal standard.

\section{Yield determination:}

In a dry, $\mathrm{N}_{2}$-filled glovebox, $16(25.4 \mathrm{mg}, 29.0 \mu \mathrm{mol}, 1.00$ equiv. $)$ and dichloromethane- $d_{2}(0.5 \mathrm{~mL})$ were added to a $4 \mathrm{~mL}$ scintillation vial to form a clear colorless solution. The vial was cooled to $-45{ }^{\circ} \mathrm{C}$ in a $\mathrm{CO}_{2} /{ }^{i} \mathrm{PrOH}$-cooled cold well for $30 \mathrm{~min}$. and methylmagnesium chloride in THF $(\mathrm{c}=3.33 \mathrm{M}, 35.7 \mu \mathrm{L}$, 0.119 mmol, 4.00 equiv.) was added. The reaction vial was sealed with a Teflon-lined cap and the vial was removed from the cold well and shaken for 90 seconds at $23{ }^{\circ} \mathrm{C}$ to dissolve frozen droplets of Grignard reagent. The solution was stirred at $-45^{\circ} \mathrm{C}$ for 3 hours, then at $23{ }^{\circ} \mathrm{C}$ for $30 \mathrm{~min}$., at which time precipitation of a white solid was observed. The reaction vial was stirred at $-45^{\circ} \mathrm{C}$ for $30 \mathrm{~min}$. Butadiene $(20 \mu \mathrm{L})$ and triethoxysilane $(11.4 \mu \mathrm{L}, 10.1 \mathrm{mg}, 62.0 \mu \mathrm{mol}, 2.00$ equiv.) were added and the reaction stood at $23{ }^{\circ} \mathrm{C}$ for 3 hours. The vial was unsealed and 1,5-cyclooctadiene (internal standard, $7.2 \mu \mathrm{L}, 6.4 \mathrm{mg}, 59$ $\mu$ mol, 2.0 equiv.) was added. The vial contents were thoroughly mixed and transferred to an NMR tube which was sealed with a plastic cap and electrical tape. ${ }^{1} \mathrm{H}$ NMR analysis showed the title compound, 19 , in $85 \%$ yield relative to the internal standard.

NMR Spectroscopy: ${ }^{1} \mathrm{H}$ NMR (400 MHz, $\mathrm{CD}_{2} \mathrm{Cl}_{2}, 25{ }^{\circ} \mathrm{C}, \delta$ ): 4.02 (s, br, 12H), 3.90 (q, 9H) 1.95 (s, br, $12 \mathrm{H}), 1.49\left(\mathrm{~d},{ }^{3} J_{\mathrm{P}-\mathrm{H}}=11.2 \mathrm{~Hz}, 6 \mathrm{H}\right), 1.43\left(\mathrm{~d},{ }^{3} J_{\mathrm{P}-\mathrm{H}}=11.2 \mathrm{~Hz}, 18 \mathrm{H}\right), 0.36\left(\mathrm{~d},{ }^{3} J_{\mathrm{P}-\mathrm{H}}=4.7 \mathrm{~Hz},{ }^{2} J_{\mathrm{Pt}-\mathrm{H}}=47.0\right.$ $\mathrm{Hz}, 3 \mathrm{H}), 1.25(\mathrm{t}, 9 \mathrm{H}), 0.63\left(\mathrm{~d},{ }^{3} J_{\mathrm{P}-\mathrm{H}}=14.7 \mathrm{~Hz},{ }^{2} J_{\mathrm{Pt}-\mathrm{H}}=68.1 \mathrm{~Hz}\right), 0.33\left(\mathrm{~d},{ }^{3} J_{\mathrm{P}-\mathrm{H}}=5.28 \mathrm{~Hz},{ }^{2} J_{\mathrm{Pt}-\mathrm{H}}=66.9\right.$ Hz). ${ }^{13} \mathrm{C}$ NMR (125 MHz, $\left.\mathrm{CD}_{2} \mathrm{Cl}_{2}, 25{ }^{\circ} \mathrm{C}, \delta\right): 69.7,57.8,36.2\left(\mathrm{~s}, J_{\mathrm{Pt}-\mathrm{C}}=38.2 \mathrm{~Hz}\right), 34.9,32.4,31.9,17.9$, 17.7. ${ }^{31} \mathrm{P}$ NMR $\left(162 \mathrm{MHz}, \mathrm{CD}_{2} \mathrm{Cl}_{2}, 25^{\circ} \mathrm{C}, \delta\right): 16.6 \mathrm{ppm}\left({ }^{1} J_{\mathrm{P}-\mathrm{Pt}}=1421 \mathrm{~Hz}\right)$.

Preparation of $x$-ray quality crystals:

Single crystals suitable for $\mathrm{x}$-ray diffraction were grown in a dry, $\mathrm{N}_{2}$-filled glovebox from a saturated acetonitrile or THF/hexanes solution of 19. THF/hexanes conditions: in a $4 \mathrm{~mL}$ scintillation vial, 19 (25.0 $\mathrm{mg}$ ) was dissolved in THF $(0.5 \mathrm{~mL})$ and the resulting clear solution was filtered through a plug of glass 
wool and diluted with hexanes $(3 \mathrm{~mL})$ before cooling at $-30{ }^{\circ} \mathrm{C}$ for one week, forming block-like colorless crystals. Acetonitrile conditions: in a $4 \mathrm{~mL}$ scintillation vial, 19 (50.0 mg) was suspended in $\mathrm{MeCN}(0.5 \mathrm{~mL})$ to form a suspension. The suspension was filtered through glass wool to remove undissolved solids and cooled at $-30{ }^{\circ} \mathrm{C}$ for 4 weeks to yield colorless needle-like crystals. X-ray data are included in x-ray data analysis section.

$\left[\left(\kappa^{2}-\left({ }^{t} \mathrm{Bu}\right)_{2} \mathrm{PCH}_{2} \mathrm{CMe}_{2} \mathrm{CH}_{2}\right) \mathrm{PtCl}_{2}{ }^{11}(40)\right.$
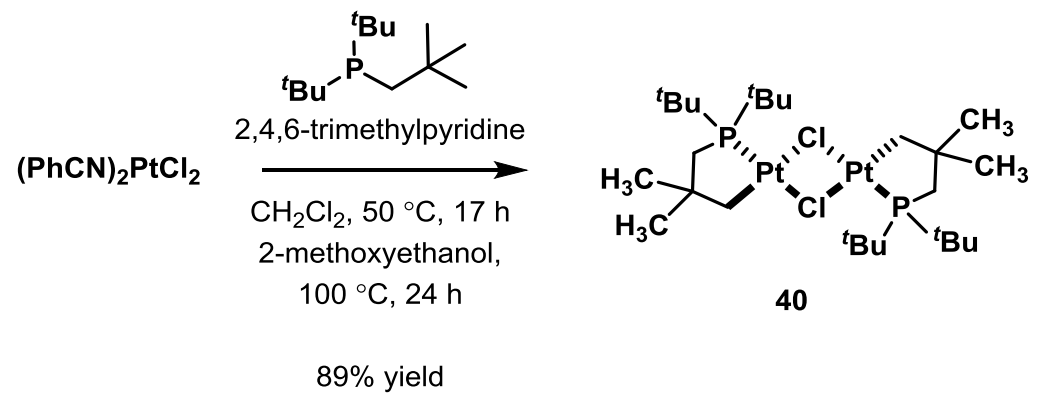

Preparation of 40 was adapted from the method of Mason et al. ${ }^{11}$ In a dry, $\mathrm{N}_{2}$-filled glovebox, $(\mathrm{PhCN})_{2} \mathrm{PtCl}_{2}$ (441 mg, $0.934 \mathrm{mmol}, 1.00$ equiv.), dichloromethane (1.9 mL), and a Teflon-coated magnetic stirring bar were added to a $20 \mathrm{~mL}$ scintillation vial at $23{ }^{\circ} \mathrm{C}$, forming a yellow suspension. Ditert-butylneopentylphosphine (241 $\mu \mathrm{L}, 202 \mathrm{mg}, 0.934 \mathrm{mmol}, 1.00$ equiv.) and 2,4,6-trimethylpyridine (124 $\mu \mathrm{L}, 114 \mathrm{mg}, 0.934 \mathrm{mmol}, 1.00$ equiv.) were added. The reaction vial was sealed with a polyethylene-lined cap and removed from the glovebox. The reaction mixture was stirred at $50{ }^{\circ} \mathrm{C}$ in an aluminum heating block for 16.5 hours, cooled at $23{ }^{\circ} \mathrm{C}$ for $5 \mathrm{~min}$., and opened under ambient atmosphere. The solvent was removed by rotary evaporation, then 2-methoxyethanol $(1.9 \mathrm{~mL})$ was added and the resulting suspension was stirred at $100{ }^{\circ} \mathrm{C}$ for 24.5 hours. Centrifugation followed by decantation of the supernatant yielded a residue that was washed with methanol $(2 \times 1 \mathrm{~mL})$ and hexanes $(1 \mathrm{~mL})$ to afford the title compound as a colorless solid (370. $\mathrm{mg}, 0.415 \mathrm{mmol}, 89 \%$ yield).

${ }^{11}$ Mason, R.; Textor, M.; Al-Salem N.; Shaw, B. Chem. Commun.1976, 292-293. 
NMR Spectroscopy: ${ }^{1} \mathrm{H}$ NMR $\left(400 \mathrm{MHz}, \mathrm{CD}_{2} \mathrm{Cl}_{2}, 25{ }^{\circ} \mathrm{C}, \delta\right): 2.00\left(\mathrm{~d},{ }^{2} J_{\mathrm{Pt}-\mathrm{H}}=105 \mathrm{~Hz}, 4 \mathrm{H}\right), 1.73\left(\mathrm{~d},{ }^{2} J_{\mathrm{P}-\mathrm{H}}=\right.$ $8.72 \mathrm{~Hz}, 4 \mathrm{H}), 1.40\left(\mathrm{~d},{ }^{3} \mathrm{~J}_{\mathrm{P}-\mathrm{H}}=13.7 \mathrm{~Hz}, 36 \mathrm{H}\right), 1.21(\mathrm{~s}, 12 \mathrm{H}) .{ }^{13} \mathrm{C} \mathrm{NMR}\left(125 \mathrm{MHz}, \mathrm{CD}_{2} \mathrm{Cl}_{2}, 25{ }^{\circ} \mathrm{C}, \delta\right): 42.9$ $\left(\mathrm{d}, J_{\mathrm{P}-\mathrm{C}}=7.60 \mathrm{~Hz}\right), 37.0\left(\mathrm{~d}, J_{\mathrm{P}-\mathrm{C}}=32.1 \mathrm{~Hz}\right), 35.0\left(\mathrm{~d}, J_{\mathrm{P}-\mathrm{C}}=27.5 \mathrm{~Hz}\right), 31.9\left(\mathrm{~d}, J_{\mathrm{P}-\mathrm{C}}=8.82 \mathrm{~Hz}\right), 31.2,29.6-$ 29.5 (m). ${ }^{31} \mathrm{P}$ NMR (162 MHz, $\left.\mathrm{CD}_{2} \mathrm{Cl}_{2}, 25{ }^{\circ} \mathrm{C}, \delta\right): 69.7\left({ }^{1} J_{\mathrm{P}-\mathrm{Pt}}=4977 \mathrm{~Hz}\right), 69.5\left({ }^{1} J_{\mathrm{P}-\mathrm{Pt}}=5005 \mathrm{~Hz}\right)$. Anal: calcd for $\mathrm{C}_{26} \mathrm{H}_{56} \mathrm{Cl}_{2} \mathrm{P}_{2} \mathrm{Pt}_{2}$ : C, 35.02; H, 6.33; found: C, 35.05; H, 6.09. Spectroscopic data shows the title compound as a 1:2.5 mixture of head-to-head and head-to-tail dimeric structures.

\section{$\left[\left(\kappa^{2}-\left({ }^{t} \mathrm{Bu}\right)_{2} \mathrm{PCH}_{2} \mathrm{CMe}_{2} \mathrm{CH}_{2}\right) \operatorname{Pt}(\mathrm{MeCN})_{2}\right][\mathrm{OTf}](41)$}

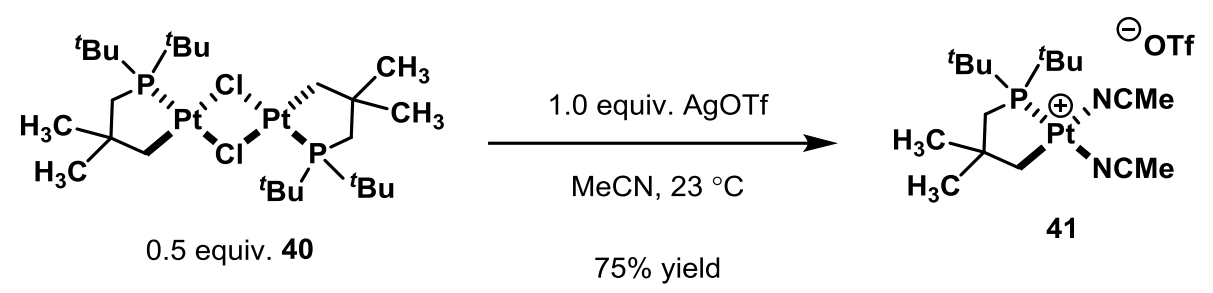

Platinum complex 40 (250 mg, 0.280 mmol, 1.00 equiv.), AgOTf (144 mg, 0.560 mmol, 2.00 equiv.), and a Teflon-coated magnetic stirring bar were added to a $20 \mathrm{~mL}$ amber-colored scintillation vial. Acetonitrile $(5.5 \mathrm{~mL})$ was added at $23{ }^{\circ} \mathrm{C}$ to form a white suspension, which was sealed with a Teflon-lined cap and stirred at $23{ }^{\circ} \mathrm{C}$ for 12 hours. The vial was opened and the white suspension filtered through a plug of glass wool to yield a clear, colorless solution. The solvent was removed by rotary evaporation and dichloromethane $(2 \mathrm{~mL})$ was added. The resulting suspension was filtered through a plug of celite and the colorless filtrate was concentrated by rotary evaporation. The colorless solid was purified by recrystallization from a saturated solution in acetonitrile/diethyl ether cooled at $-30{ }^{\circ} \mathrm{C}$ to yield the title compound as a white powder (269 mg, $0.419 \mathrm{mmol}, 75 \%)$.

NMR Spectroscopy: ${ }^{1} \mathrm{H}$ NMR (400 MHz, $\left.\mathrm{CD}_{2} \mathrm{Cl}_{2}, 25{ }^{\circ} \mathrm{C}, \delta\right): 2.42(\mathrm{~s}, 3 \mathrm{H}), 2.34(\mathrm{~s}, 3 \mathrm{H}), 1.95\left(\mathrm{~d},{ }^{2} J_{\mathrm{Pt}-\mathrm{H}}=\right.$ $95.9 \mathrm{~Hz}, 2 \mathrm{H}), 1.79\left(\mathrm{~d},{ }^{2} J_{\mathrm{P}-\mathrm{H}}=9.19 \mathrm{~Hz}, 2 \mathrm{H}\right), 1.35\left(\mathrm{~d},{ }^{3} J_{\mathrm{P}-\mathrm{H}}=14.1 \mathrm{~Hz}, 18 \mathrm{H}\right), 1.18(\mathrm{~s}, 6 \mathrm{H}) .{ }^{13} \mathrm{C} \mathrm{NMR}(125$ $\left.\mathrm{MHz}, \mathrm{CD}_{2} \mathrm{Cl}_{2}, 25^{\circ} \mathrm{C}, \delta\right): 125.5\left(\mathrm{~d}, J_{\mathrm{Pt}-\mathrm{C}}=321 \mathrm{~Hz}\right), 119.4\left(\mathrm{~d}, J_{\mathrm{P}-\mathrm{C}}=15.5 \mathrm{~Hz}\right), 43.3\left(\mathrm{~d}, J_{\mathrm{P}-\mathrm{C}}=6.51 \mathrm{~Hz}\right)$, $36.6\left(\mathrm{~d}, J_{\mathrm{P}-\mathrm{C}}=33.2 \mathrm{~Hz}\right), 35.4\left(\mathrm{~d}, J_{\mathrm{P}-\mathrm{C}}=28.4 \mathrm{~Hz}\right), 35.4\left(\mathrm{~d}, J_{\mathrm{P}-\mathrm{C}}=28.4 \mathrm{~Hz}\right), 32.6\left(\mathrm{~d}, J_{\mathrm{P}-\mathrm{C}}=9.05 \mathrm{~Hz}\right), 29.8$ 
$\left(\mathrm{d}, J_{\mathrm{P}-\mathrm{C}}=3.05 \mathrm{~Hz}\right), 28.4 .{ }^{31} \mathrm{P} \mathrm{NMR}\left(162 \mathrm{MHz}, \mathrm{CD}_{2} \mathrm{Cl}_{2}, 25{ }^{\circ} \mathrm{C}, \delta\right): 69.4\left({ }^{1} J_{\mathrm{P}-\mathrm{Pt}}=4604 \mathrm{~Hz}\right) .{ }^{19} \mathrm{~F} \mathrm{NMR}(376$

$\left.\mathrm{MHz}, \mathrm{CD}_{2} \mathrm{Cl}_{2}, 25^{\circ} \mathrm{C}, \delta\right):-78.8$. Anal: calcd for $\mathrm{C}_{38} \mathrm{H}_{34} \mathrm{~F}_{3} \mathrm{~N}_{2} \mathrm{O}_{3}$ PPtS: C, 33.70; $\mathrm{H}, 5.34 ; \mathrm{N}, 4.37$; found: $\mathrm{C}$, $33.84 ; \mathrm{H}, 5.22 ; \mathrm{N}, 4.28$.

$\left[\left(\kappa^{2}-\left({ }^{t} \mathrm{Bu}\right)_{2} \mathrm{PCH}_{2} \mathrm{CMe}_{2} \mathrm{CH}_{2}\right) \mathrm{Pt}(\mathrm{Me})_{2}\right]\left[\mathrm{MgCl}(\mathrm{THF})_{3}\right](37)$
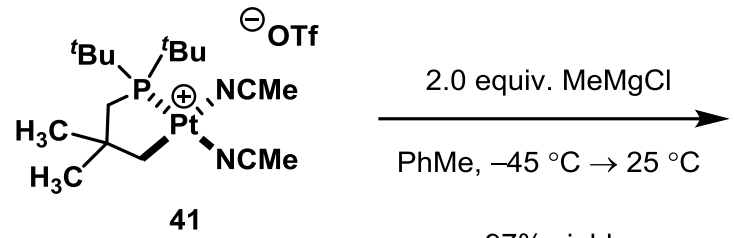

$97 \%$ yield

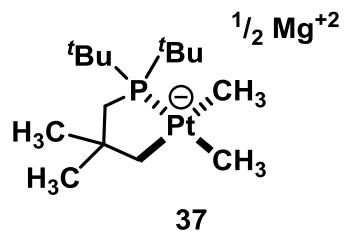

In a dry, $\mathrm{N}_{2}$-filled glovebox, platinum complex 41 (97.5 mg, $0.152 \mathrm{mmol}, 1.00$ equiv.), toluene (1.0 mL) and a Teflon-coated magnetic stirring bar were added to a $4 \mathrm{~mL}$ scintillation vial, forming a white suspension. The mixture was stirred at $-45{ }^{\circ} \mathrm{C}$ in a $\mathrm{CO}_{2} /{ }^{i} \mathrm{PrOH}-$ cooled cold well for $30 \mathrm{~min}$. and methylmagnesium chloride in THF (c = 3.28 M, 93.0 $\mu \mathrm{L}, 0.304$ mmol, 2.00 equiv.) was added. The reaction vial was sealed with a Teflon-lined cap and the vial was removed from the cold well and shaken for 90 seconds to dissolve frozen droplets of the Grignard reagent. The suspension was stirred at $-45^{\circ} \mathrm{C}$ for 2 hours, then at $-30{ }^{\circ} \mathrm{C}$ in the glovebox freezer for 14 hours, at which point precipitation of a dark solid was observed. Filtration through glass wool afforded a clear colorless solution which was concentrated in vacuo to yield the title compound as a colorless solid (103 mg) containing the product and aggregated $\mathrm{MgCl}_{2}(\mathrm{THF})_{2}$. The product could not be purified by recrystallization due to the high solubility of $\mathbf{3 7}$ in organic solvents and the high lattice energy of $\mathrm{MgCl}_{2}(\mathrm{THF})_{2}$. Attempts to purify $\mathbf{3 7}$ by crystallization yielded decomposition of $\mathbf{3 7}$ and crystalline magnesium salts. The tendency of 37 to aggregate with $\mathrm{MgCl}_{2}(\mathrm{THF})_{2}$ and its resistance to further purification by recrystallization prevented us from obtaining satisfactory results from elemental microanalysis. Reaction yield and purity were determined in a separate experiment from ${ }^{1} \mathrm{H}$ NMR integrations relative to an internal standard. 


\section{Yield determination:}

In a dry, $\mathrm{N}_{2}$-filled glovebox, platinum complex 41 (31.3 mg, $48.9 \mu \mathrm{mol}, 1.00$ equiv.), toluene- $d_{8}(0.5 \mathrm{~mL})$, and a Teflon-coated magnetic stirring bar were added to a $4 \mathrm{~mL}$ scintillation vial at $23{ }^{\circ} \mathrm{C}$. The suspension was stirred at $-45{ }^{\circ} \mathrm{C}$ in a $\mathrm{CO}_{2} /{ }^{i} \mathrm{PrOH}$-cooled cold well for $30 \mathrm{~min}$. and methylmagnesium chloride in THF (c = 3.33 M, $29.4 \mu \mathrm{L}, 97.8 \mu \mathrm{mol}, 2.00$ equiv.) was added. The reaction vial was sealed with a Teflon-lined cap and the vial was shaken for 90 seconds at $23{ }^{\circ} \mathrm{C}$ to dissolve frozen droplets of Grignard reagent. The resulting suspension was stirred at $-45{ }^{\circ} \mathrm{C}$ for 2 hours, then at $-30{ }^{\circ} \mathrm{C}$ for 8 hours in the glovebox freezer. The vial was unsealed and 1,5-cyclooctadiene (internal standard, $10.0 \mu \mathrm{L}, 8.82 \mathrm{mg}$, $81.0 \mu \mathrm{mol}, 1.66$ equiv.) was added. The suspension was thoroughly mixed and transferred to an NMR tube which was sealed with a plastic cap and electrical tape. ${ }^{1} \mathrm{H}$ NMR analysis showed the title compound, 37, in $97 \%$ yield relative to the internal standard.

NMR Spectroscopy: ${ }^{1} \mathrm{H}$ NMR $\left(400 \mathrm{MHz}, \mathrm{CD}_{2} \mathrm{Cl}_{2}, 25^{\circ} \mathrm{C}, \delta\right): 4.02(\mathrm{~s}, \mathrm{br}, 8 \mathrm{H}), 1.93(\mathrm{~s}, \mathrm{br}, 8 \mathrm{H}) 1.81\left(\mathrm{~d},{ }^{2} J_{\mathrm{P}-}\right.$ $\mathrm{H}=7.70 \mathrm{~Hz}, 2 \mathrm{H}), 1.26\left(\mathrm{~d},{ }^{3} J_{\mathrm{P}-\mathrm{H}}=12.1 \mathrm{~Hz}, 18 \mathrm{H}\right), 1.20-0.92(\mathrm{~m}, 8 \mathrm{H}), 0.42\left(\mathrm{~d},{ }^{3} J_{\mathrm{P}-\mathrm{H}}=3.91 \mathrm{~Hz},{ }^{2} J_{\mathrm{Pt}-\mathrm{H}}=43.3\right.$ $\mathrm{Hz}, 3 \mathrm{H}), 0.19\left(\mathrm{~d},{ }^{2} J_{\mathrm{Pt}-\mathrm{H}}=28.0 \mathrm{~Hz}, 3 \mathrm{H}\right) .{ }^{13} \mathrm{C} \mathrm{NMR}\left(100 \mathrm{MHz}, \mathrm{CD}_{2} \mathrm{Cl}_{2}, 25{ }^{\circ} \mathrm{C}, \delta\right): 70.2,49.9\left(\mathrm{~d}, J_{\mathrm{Pt}-\mathrm{C}}=570\right.$ $\mathrm{Hz}), 43.7\left(\mathrm{~d}, J_{\mathrm{P}-\mathrm{C}}=14.6 \mathrm{~Hz}\right), 41.2\left(\mathrm{~d}, J_{\mathrm{P}-\mathrm{C}}=26.3 \mathrm{~Hz}\right), 35.0\left(\mathrm{~d}, J_{\mathrm{P}-\mathrm{C}}=6.10 \mathrm{~Hz}\right), 30.3\left(\mathrm{~d}, J_{\mathrm{P}-\mathrm{C}}=6.48 \mathrm{~Hz}\right)$, $25.8,-3.47\left(J_{\mathrm{P}-\mathrm{C}}=106 \mathrm{~Hz}\right),-13.9 .{ }^{31} \mathrm{P} \mathrm{NMR}\left(162 \mathrm{MHz}, \mathrm{CD}_{2} \mathrm{Cl}_{2}, 25^{\circ} \mathrm{C}, \delta\right): 70.8\left({ }^{1} J_{\mathrm{P}-\mathrm{Pt}}=2412 \mathrm{~Hz}\right)$.

\section{$\left[\left(\kappa^{2}-\left({ }^{t} \mathrm{Bu}\right)_{2} \mathrm{PCMe}_{2} \mathrm{CH}_{2}\right) \mathrm{Pt}(\mathrm{NCMe})_{2}\right](44)$}

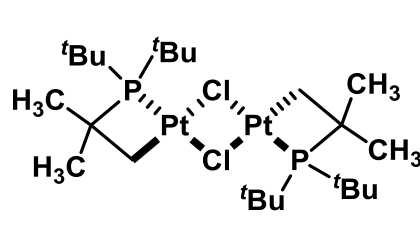

0.5 equiv. 16

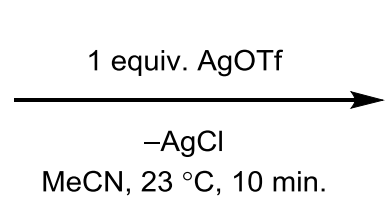

$96 \%$ yield

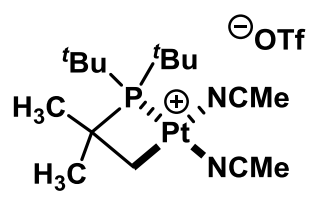

44

In a dry, $\mathrm{N}_{2}$-filled glovebox, platinum complex 16 (0.500 g, 0.579 mmol, 1.00 equiv.), AgOTf (0.297 g, $1.16 \mathrm{mmol}, 2.00$ equiv.), a Teflon-coated magnetic stirring bar, and acetonitrile $(10 \mathrm{~mL})$ were added to a $20 \mathrm{~mL}$ scintillation vial at $23{ }^{\circ} \mathrm{C}$ to form a white suspension. After 10 minutes, the suspension was filtered 
through glass wool to yield a clear, colorless filtrate. Concentration of the filtrate yielded the title compound as a colorless crystalline solid $(0.701 \mathrm{~g}, 1.12 \mathrm{mmol}, 96 \%)$. The title compound was used as obtained, but could be further purified by recrystallization. A saturated solution of 44 in $\mathrm{MeCN}$ at $23{ }^{\circ} \mathrm{C}$ was layered with $\mathrm{Et}_{2} \mathrm{O}$ and cooled at $-30{ }^{\circ} \mathrm{C}$ for 24 hours to yield colorless crystals.

NMR Spectroscopy: ${ }^{1} \mathrm{H}$ NMR $\left(600 \mathrm{MHz}, \mathrm{CD}_{2} \mathrm{Cl}_{2}, 25{ }^{\circ} \mathrm{C}, \delta\right): 2.38(\mathrm{~s}, 3 \mathrm{H}), 2.34(\mathrm{~s}, 3 \mathrm{H}), 1.48\left(\mathrm{~d},{ }^{3} J_{\mathrm{P}-\mathrm{H}}=\right.$ 14.1 Hz, 18H), $1.47\left(\mathrm{~d},{ }^{3} \mathrm{~J}_{\mathrm{P}-\mathrm{H}}=14.7 \mathrm{~Hz}, 6 \mathrm{H}\right), 1.37\left(\mathrm{~d},{ }^{3} \mathrm{~J}_{\mathrm{P}-\mathrm{H}}=8.2 \mathrm{~Hz}, 2 \mathrm{H}\right) .{ }^{13} \mathrm{C} \mathrm{NMR}\left(100 \mathrm{MHz}, \mathrm{CD}_{2} \mathrm{Cl}_{2}\right.$, $\left.25{ }^{\circ} \mathrm{C}, \delta\right): 122.8,120.1,119.2,119.1,37.5\left(\mathrm{~d}, J_{\mathrm{P}-\mathrm{C}}=18.4 \mathrm{~Hz}\right), 31.7,31.1,3.1\left(\mathrm{~d}, J_{\mathrm{P}-\mathrm{C}}=9.2 \mathrm{~Hz}\right), 3.0,3.3$ $\left(\mathrm{d},{ }^{2} J_{\mathrm{P}-\mathrm{C}}=26.1 \mathrm{~Hz}\right)$. One resonance was not observed. ${ }^{31} \mathrm{P}$ NMR $\left(162 \mathrm{MHz}, \mathrm{CD}_{2} \mathrm{Cl}_{2}, 25{ }^{\circ} \mathrm{C}, \delta\right):-17.5\left({ }^{1} J_{\mathrm{P}-}\right.$ $\mathrm{Pt}=3451 \mathrm{~Hz}) .{ }^{19} \mathrm{~F}$ NMR $\left(376 \mathrm{MHz}, \mathrm{CD}_{2} \mathrm{Cl}_{2}, 25^{\circ} \mathrm{C}, \delta\right):-78.9$. Anal: calcd for $\mathrm{C}_{17} \mathrm{H}_{32} \mathrm{~F}_{3} \mathrm{~N}_{2} \mathrm{O}_{3} \mathrm{PPtS}$ : C, 32.54; H, 5.14; N, 4.46; found: C, 32.63; H, 5.01; N, 4.48 .

\section{$\left[\left(\kappa^{2}-\left({ }^{t} \mathrm{Bu}\right)_{2} \mathrm{PCMe}_{2} \mathrm{CH}_{2}\right) \mathrm{PtCH}_{3}(\mathrm{NCMe})\right](45)$}

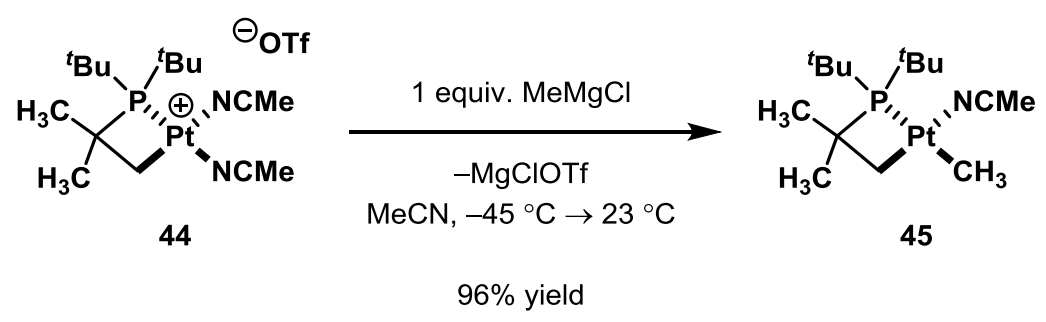

In a dry, $\mathrm{N}_{2}$-filled glovebox, platinum complex 44 (50.0 mg, $80.0 \mu \mathrm{mol}, 1.00$ equiv.), a Teflon-coated magnetic stirring bar, and acetonitrile $(1.0 \mathrm{~mL})$ were added to a $20 \mathrm{~mL}$ scintillation vial at $23{ }^{\circ} \mathrm{C}$ to form a clear, colorless solution. The solution was cooled to $-40{ }^{\circ} \mathrm{C}$ in a $\mathrm{CO}_{2} /{ }^{i} \mathrm{PrOH}-$ cooled cold well for $30 \mathrm{~min}$. and methylmagnesium chloride in THF ( $\mathrm{c}=3.25 \mathrm{M}, 24.6 \mu \mathrm{L}, 0.080 \mathrm{mmol}, 1.00$ equiv.) was added. The reaction vial was sealed with a polyethylene-lined cap and shaken for 90 seconds at $23{ }^{\circ} \mathrm{C}$ to dissolve frozen droplets of Grignard reagent. The resulting solution was stirred at $-40{ }^{\circ} \mathrm{C}$ for 15 minutes, then at $23{ }^{\circ} \mathrm{C}$ for $15 \mathrm{~min}$. The vial was opened and the solution diluted with diethyl ether $(6.0 \mathrm{~mL})$, then pentane $(6.0 \mathrm{~mL})$, at which time a white precipitate was observed. Filtration through glass wool yielded a clear, 
colorless solution. Evaporation of the solvent in vacuo afforded the title compound as a colorless crystalline solid (34.6 mg, 96\% yield).

NMR Spectroscopy: ${ }^{1} \mathrm{H}$ NMR (400 MHz, $\left.\mathrm{CD}_{3} \mathrm{CN}, 23{ }^{\circ} \mathrm{C}, \delta\right): 1.96(\mathrm{~s}, 3 \mathrm{H}), 1.45-1.37(\mathrm{~m}, 24 \mathrm{H}), 1.14(\mathrm{~d}$, $\left.{ }^{3} J_{\mathrm{P}-\mathrm{H}}=14.4 \mathrm{~Hz},{ }^{2} J_{\mathrm{Pt}-\mathrm{H}}=98.3 \mathrm{~Hz}, 2 \mathrm{H}\right),-0.01\left(\mathrm{~d},{ }^{3} J_{\mathrm{P}-\mathrm{H}}=7.4 \mathrm{~Hz},{ }^{2} J_{\mathrm{Pt}-\mathrm{H}}=71.4 \mathrm{~Hz}, 3 \mathrm{H}\right) .{ }^{13} \mathrm{C} \mathrm{NMR}(125$ $\left.\mathrm{MHz}, \mathrm{CD}_{3} \mathrm{CN}, 25^{\circ} \mathrm{C}, \delta\right): 55.2\left(\mathrm{~d}, J_{\mathrm{P}-\mathrm{C}}=20.7 \mathrm{~Hz}\right), 37.0,33.6\left(\mathrm{~s}, J_{\mathrm{Pt}-\mathrm{C}}=60.9 \mathrm{~Hz}\right), 32.1\left(\mathrm{~d}, J_{\mathrm{P}-\mathrm{C}}=4.6 \mathrm{~Hz}\right)$, $4.6\left(\mathrm{~d}, J_{\mathrm{P}-\mathrm{C}}=17.8 \mathrm{~Hz}\right),-2.3\left(\mathrm{~d}, J_{\mathrm{P}-\mathrm{C}}=105.2 \mathrm{~Hz}\right) .{ }^{31} \mathrm{P} \mathrm{NMR}\left(162 \mathrm{MHz}, \mathrm{CD}_{3} \mathrm{CN}, 25{ }^{\circ} \mathrm{C}, \delta\right): 21.8\left({ }^{1} J_{\mathrm{P}-\mathrm{Pt}}=\right.$ $1359 \mathrm{~Hz}$ ). Anal: calcd for $\mathrm{C}_{15} \mathrm{H}_{32} \mathrm{NPPt}$ C, 39.82; H, 7.13; N, 3.10; found: C, 39.58; H, 6.87; N, 3.05 .

\section{$\left[\left(\kappa^{2}-\left({ }^{t} \mathrm{Bu}\right)_{2} \mathrm{PCMe} 2 \mathrm{CH}_{2}\right) \operatorname{Pt}\left(\mathrm{Si}(\mathrm{OEt})_{3}\right)(\mathrm{NCMe})\right](42)$}
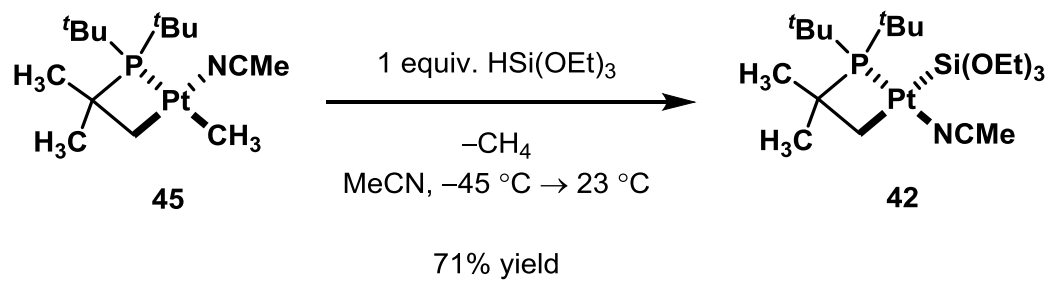

In a dry, $\mathrm{N}_{2}$-filled glovebox, platinum complex 45 (100.0 mg, $0.2210 \mathrm{mmol}, 1.000$ equiv.), a Tefloncoated magnetic stirring bar, and acetonitrile $(2.0 \mathrm{~mL})$ were added to a $20 \mathrm{~mL}$ scintillation vial at $23{ }^{\circ} \mathrm{C}$. The clear, colorless solution was cooled at $-40{ }^{\circ} \mathrm{C}$ in a $\mathrm{CO}_{2} / \mathrm{PrOH}$-cooled cold well for $30 \mathrm{~min}$. Triethoxysilane ( $40.8 \mu \mathrm{L}, 36.3 \mathrm{mg}, 0.221 \mathrm{mmol}, 1.00$ equiv.) was added and the solution was stirred at 23 ${ }^{\circ} \mathrm{C}$ for 1 hour, then cooled to $-30{ }^{\circ} \mathrm{C}$ for 14 hours in the glovebox freezer, at which time a tan-colored crystalline precipitate was observed. The supernatant was decanted and the crystals were washed with cold MeCN $(2 \times 0.2 \mathrm{~mL})$ and dried in vacuo to yield the title compound as a tan-colored crystalline solid (97.4 mg, $0.162 \mathrm{mmol}, 73 \%$ ). After solvent removal, continued exposure of the product to vacuum resulted in discoloration and decomposition (observed by ${ }^{1} \mathrm{H}$ and ${ }^{31} \mathrm{P}$ NMR). Although obtained as a single isomer, the title compound isomerizes rapidly in solution at $23{ }^{\circ} \mathrm{C}$ to form the diastereomer in which the silicon ligand is trans to the phosphine. 
NMR Spectroscopy: ${ }^{1} \mathrm{H}$ NMR (600 MHz, $\left.\mathrm{CD}_{3} \mathrm{CN}, 23{ }^{\circ} \mathrm{C}, \delta\right): 3.74$ (q, $\left.J=48.1 \mathrm{~Hz}, 6 \mathrm{H}\right), 1.96(\mathrm{~s}, 3 \mathrm{H}), 1.46$ $\left(\mathrm{d},{ }^{3} J_{\mathrm{P}-\mathrm{H}}=13.5 \mathrm{~Hz}, 18 \mathrm{H}\right), 1.42\left(\mathrm{~d},{ }^{3} J_{\mathrm{P}-\mathrm{H}}=13.5 \mathrm{~Hz}, 6 \mathrm{H}\right), 1.11(\mathrm{t}, J=6.8 \mathrm{~Hz}, 9 \mathrm{H}), 0.58\left(\mathrm{~d},{ }^{3} J_{\mathrm{P}-\mathrm{H}}=10.6 \mathrm{~Hz}\right.$, $\left.{ }^{2} J_{\mathrm{Pt}-\mathrm{H}}=48.1 \mathrm{~Hz}, 2 \mathrm{H}\right) .{ }^{13} \mathrm{C} \mathrm{NMR}\left(125 \mathrm{MHz}, \mathrm{CD}_{3} \mathrm{CN}, 25{ }^{\circ} \mathrm{C}, \delta\right): 57.0,54.6\left(\mathrm{~d}, J_{\mathrm{P}-\mathrm{C}}=35.0 \mathrm{~Hz}\right), 37.1\left(\mathrm{~d}, J_{\mathrm{P}-\mathrm{C}}\right.$ $=14.9 \mathrm{~Hz}), 35.9\left(\mathrm{~s}, J_{\mathrm{Pt}-\mathrm{C}}=45.5 \mathrm{~Hz}\right), 31.8,28.1\left(\mathrm{~s}, J_{\mathrm{P}-\mathrm{C}}=28.8 \mathrm{~Hz}\right), 19.4\left(\mathrm{~d}, J_{\mathrm{P}-\mathrm{C}}=22.0 \mathrm{~Hz}\right)$. One signal not observed. ${ }^{31} \mathrm{P}$ NMR $\left(162 \mathrm{MHz}, \mathrm{CD}_{3} \mathrm{CN}, 25{ }^{\circ} \mathrm{C}, \delta\right):-3.4\left({ }^{1} J_{\mathrm{P}-\mathrm{Pt}}=3578 \mathrm{~Hz}\right)$. Anal: calcd for $\mathrm{C}_{20} \mathrm{H}_{44} \mathrm{NO}_{3} \mathrm{PPtSi}: \mathrm{C}, 39.99 ; \mathrm{H}, 7.38 ; \mathrm{N}, 2.33$; found: $\mathrm{C}, 39.89 ; \mathrm{H}, 7.12 ; \mathrm{N}, 2.33$.

\section{$\left[\left(\kappa^{2}-\left({ }^{t} \mathrm{Bu}\right)_{2} \mathrm{PCMe}_{2} \mathrm{CH}_{2}\right) \operatorname{Pt}\left(\mathrm{Si}(\mathrm{OEt})_{3}\right)\left(\mathrm{CH}_{2} \mathrm{CH}_{2} \mathrm{CH}=\mathrm{CH}_{2}\right)\right](36)$}
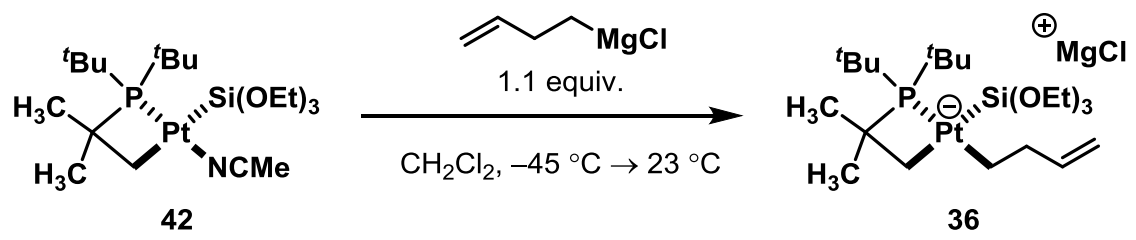

In a dry, $\mathrm{N}_{2}$-filled glovebox, platinum complex $42(25.0 \mathrm{mg}, 55.0 \mu \mathrm{mol}, 1.00$ equiv.) was added to a $4 \mathrm{~mL}$ scintillation vial and cooled at $-45{ }^{\circ} \mathrm{C}$ in a $\mathrm{CO}_{2} /{ }^{i} \mathrm{PrOH}-$ cooled cold well for $30 \mathrm{~min}$. Pre-cooled dichloromethane- $d_{2} \quad(0.5 \mathrm{~mL})$ was added at $-45{ }^{\circ} \mathrm{C}$ to form a pale yellow solution. 3Butenylmagnesiumchloride in THF ( $c=1.15 \mathrm{M}, 48.0 \mu \mathrm{L}, 55.0 \mu \mathrm{mol}, 1.00$ equiv.) was added and the reaction vial was sealed with a Teflon-lined cap and shaken at $23{ }^{\circ} \mathrm{C}$ for 90 seconds to dissolve any frozen droplets of Grignard reagent. The pale yellow solution was stirred at $-45{ }^{\circ} \mathrm{C}$ for $30 \mathrm{~min}$., then at $23{ }^{\circ} \mathrm{C}$ for 30 min. The contents of the reaction vial were transferred to a screw-capped NMR tube for analysis. Attempts to isolate 36 by diluting the reaction solution with benzene $(3 \mathrm{~mL})$ then freezing the solution at $-45{ }^{\circ} \mathrm{C}$ and lyophilizing the resulting solid in vacuo resulted in partial decomposition. The title compound was used for catalysis as obtained, without isolation.

NMR Spectroscopy: ${ }^{1} \mathrm{H}$ NMR (400 MHz, $\left.\mathrm{CD}_{2} \mathrm{Cl}_{2}, 23{ }^{\circ} \mathrm{C}, \delta\right): 6.02-5.84(\mathrm{~m}, 1 \mathrm{H}), 4.75(\mathrm{~d}, 19.5 \mathrm{~Hz}, 1 \mathrm{H})$, $4.58(\mathrm{~d}, 8.58 \mathrm{~Hz}, 1 \mathrm{H}), 3.84\left(\mathrm{q},{ }^{3} J_{\mathrm{H}-\mathrm{H}}=7.0 \mathrm{~Hz}, 6 \mathrm{H}\right), 3.73(\mathrm{~s})^{*}, 2.392 .12(\mathrm{~m}, 2 \mathrm{H}), 1.63(\mathrm{~s})^{*}, 1.511 .35(\mathrm{~m}$, $24 \mathrm{H}), 1.22\left(\mathrm{t},{ }^{3} J_{\mathrm{H}-\mathrm{H}}=7.4 \mathrm{~Hz}, 9 \mathrm{H}\right), 0.68\left(\mathrm{~d},{ }^{3} J_{\mathrm{P}-\mathrm{H}}=14.8 \mathrm{~Hz},{ }^{2} J_{\mathrm{Pt}-\mathrm{H}}=55.4 \mathrm{~Hz}, 2 \mathrm{H}\right) .{ }^{13} \mathrm{C} \mathrm{NMR}(125 \mathrm{MHz}$, 
$\left.\mathrm{CD}_{2} \mathrm{Cl}_{2}, 23{ }^{\circ} \mathrm{C}, \delta\right): 109.1,68.7^{*}, 57.8,37.9,36.6,35.2,32.6,32.3,26.1^{*}, 18.5,18.2,2.27$. One signal not observed. ${ }^{31} \mathrm{P}$ NMR $\left(162 \mathrm{MHz}, \mathrm{CD}_{2} \mathrm{Cl}_{2}, 23{ }^{\circ} \mathrm{C}, \delta\right): 13.49\left(\mathrm{~s},{ }^{1} J_{\mathrm{Pt}-\mathrm{P}}=1300 \mathrm{~Hz}\right) .{ }^{*}$ Signals correspond to THF co-solvent from in situ generation of $\mathbf{3 6}$.

\section{$\left[\kappa^{2}-\left({ }^{t} \mathrm{Bu}\right)_{2} \mathrm{P}\left(\mathrm{C}_{6} \mathrm{H}_{4}-\text { ortho- } \mathrm{CH}_{2}\right) \mathrm{PtCl}\right]_{2}(46)$}

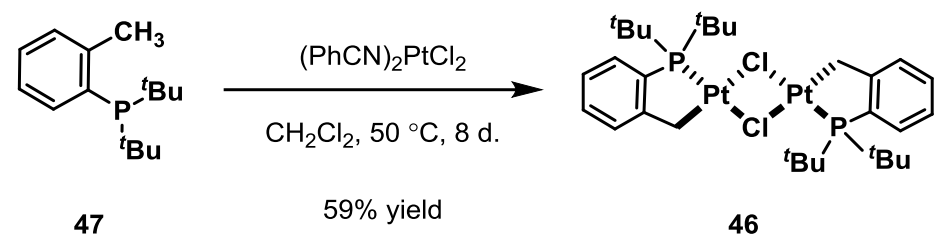

Complex 46 was synthesized by the method of Cheney and Shaw. ${ }^{12}$ In a dry, $\mathrm{N}_{2}$-filled glovebox, ligand 47 (250. mg, 1.06 mmol, 2.00 equiv.), ( $\mathrm{PhCN}_{2} \mathrm{PtCl}_{2}$ (250. mg, $0.529 \mathrm{mmol}, 1.00$ equiv.), dichloromethane $(2 \mathrm{~mL})$, and a Teflon-coated stirring bar were added to a $20 \mathrm{~mL}$ scintillation vial. The resulting solution was stirred at $23{ }^{\circ} \mathrm{C}$ for 4 days, then the vial was removed from the glovebox and heated at $50{ }^{\circ} \mathrm{C}$ in an aluminum heating block for 4 days, at which point a tan-colored solid had precipitated. The suspension was opened under ambient atmosphere, volatiles were removed under reduced pressure, and the residue was suspended in $\mathrm{EtOH}(4 \mathrm{~mL})$. Centrifugation, followed by decantation of the yellow supernatant, afforded the title compound as a tan solid which was washed with EtOH $(2 \times 10 \mathrm{~mL})$ and hexanes $(2 \times 10 \mathrm{~mL})$ and dried in vacuo $(146 \mathrm{mg}, 59 \%)$. The title compound was isolated as a 3:2 mixture of head-to-tail and head-to-head dimeric structures.

NMR Spectroscopy: ${ }^{1} \mathrm{H}$ NMR (400 MHz, $\left.\mathrm{CDCl}_{3}, 23{ }^{\circ} \mathrm{C}, \delta\right): 7.52(\mathrm{~m}, 2 \mathrm{H}), 7.34(\mathrm{~d}, J=7.8 \mathrm{~Hz}, 2 \mathrm{H}), 7.22$ $(\mathrm{t}, J=7.4 \mathrm{~Hz}, 2 \mathrm{H}), 7.07(\mathrm{t}, J=7.4 \mathrm{~Hz}, 2 \mathrm{H}), 3.45\left(\mathrm{~s},{ }^{2} J_{\mathrm{Pt}-\mathrm{H}}=114 \mathrm{~Hz}\right), 3.42\left(\mathrm{~s},{ }^{2} J_{\mathrm{Pt}-\mathrm{H}}=111 \mathrm{~Hz}\right), 1.44(\mathrm{~d}$, $\left.{ }^{3} J_{\mathrm{P}-\mathrm{H}}=14.4 \mathrm{~Hz}\right), 1.43\left(\mathrm{~d},{ }^{3} J_{\mathrm{P}-\mathrm{H}}=14.4 \mathrm{~Hz}\right) .{ }^{31} \mathrm{P} \mathrm{NMR}\left(162 \mathrm{MHz}, \mathrm{CDCl}_{3}, 23{ }^{\circ} \mathrm{C}, \delta\right): 71.6\left({ }^{1} \mathrm{~J}_{\mathrm{P}-\mathrm{Pt}}=5051 \mathrm{~Hz}\right)$, $71.5\left({ }^{1} J_{\mathrm{P}-\mathrm{Pt}}=5098 \mathrm{~Hz}\right)$. These data are consistent with previously reported spectral data.

\footnotetext{
${ }^{12}$ Cheney, A. J.; Shaw, B. L. J. Chem. Soc., Dalton Trans. 1972, 754.
} 
$\left[\kappa^{2}-\left({ }^{t} \mathrm{Bu}\right)_{2} \mathrm{P}\left(\mathrm{C}_{6} \mathrm{H}_{4}-\right.\right.$ ortho-CH2)Pt(NCMe)2][OTf] (48)

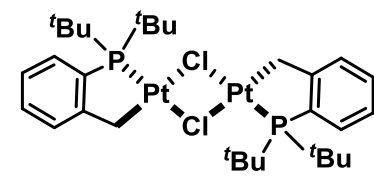

46

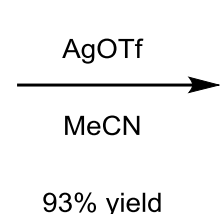

$93 \%$ yield

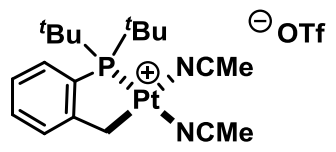

48

The synthesis of complex $\mathbf{4 8}$ was modified from the method of Cheney and Shaw. ${ }^{12}$ In a dry, $\mathrm{N}_{2}$-filled glovebox, platinum complex 46 (25.6 mg, $27.0 \mu \mathrm{mol}, 1.00$ equiv.), AgOTf (14.1 mg, $55.0 \mu \mathrm{mol}, 2.00$ equiv.), MeCN (1.0 mL), and a Teflon-coated magnetic stirring bar were added to a $20 \mathrm{~mL}$ scintillation vial. The suspension was stirred for 7 hours at $23{ }^{\circ} \mathrm{C}$, then DCM $(1.0 \mathrm{~mL})$ was added and the white suspension was filtered through a plug of glass wool. The colorless filtrate was concentrated under reduced pressure and the resulting colorless gel was triturated with $\mathrm{Et}_{2} \mathrm{O}$ and dried in vacuo to afford the title compound as a colorless solid (34.0 mg, 93\%).

NMR Spectroscopy: ${ }^{1} \mathrm{H}$ NMR (400 MHz, $\left.\mathrm{CDCl}_{3}, 23{ }^{\circ} \mathrm{C}, \delta\right): 7.55(\mathrm{t}, J=7.4 \mathrm{~Hz}, 1 \mathrm{H}), 7.38-7.30$ (m, 2H), $7.17(\mathrm{t}, J=7.4 \mathrm{~Hz}, 1 \mathrm{H}), 3.41\left(\mathrm{~s},{ }^{2} J_{\mathrm{Pt}-\mathrm{H}}=102 \mathrm{~Hz}, 2 \mathrm{H}\right), 2.47(\mathrm{~s}, 3 \mathrm{H}), 2.41(\mathrm{~s}, 3 \mathrm{H}), 1.37\left(\mathrm{~d},{ }^{2} J_{\mathrm{P}-\mathrm{H}}=14.8 \mathrm{~Hz}\right.$ 18H). ${ }^{31} \mathrm{P}$ NMR $\left(162 \mathrm{MHz}, \mathrm{CDCl}_{3}, 23{ }^{\circ} \mathrm{C}, \delta\right): 71.6\left({ }^{1} \mathrm{~J}_{\mathrm{P}-\mathrm{Pt}}=4695 \mathrm{~Hz}\right) .{ }^{19} \mathrm{~F}\left(376 \mathrm{MHz}, \mathrm{CD}_{2} \mathrm{Cl}_{3}, 23{ }^{\circ} \mathrm{C}, \delta\right)$ : -80.1 .

$\left[\kappa^{2}-\left({ }^{t} \mathrm{Bu}\right)_{2} \mathrm{P}\left(\mathrm{C}_{6} \mathrm{H}_{4}-\right.\right.$ ortho- $\left.\left.\mathrm{CH}_{2}\right) \mathrm{PtMe}_{2}\right][\mathrm{MgCl}](38)$

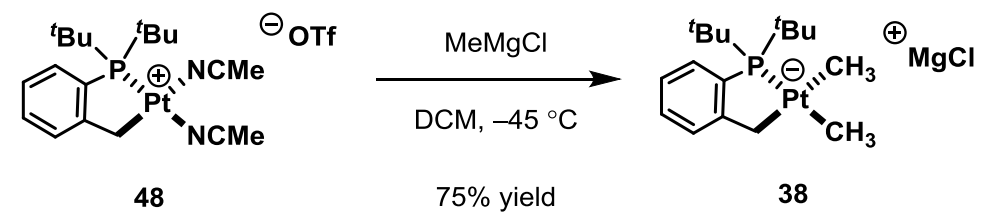

In a dry, $\mathrm{N}_{2}$-filled glovebox, platinum complex 48 (34.0 mg, $51.0 \mu \mathrm{mol}, 1.00$ equiv.), dichloromethane $(1.0 \mathrm{~mL})$, and a Teflon-coated magnetic stirring bar were added to a $4 \mathrm{~mL}$ glass scintillation vial at $23{ }^{\circ} \mathrm{C}$. The vial was chilled at $-45{ }^{\circ} \mathrm{C}$ in a $\mathrm{CO}_{2} / \mathrm{PrOH}-$ cooled cold well for $20 \mathrm{~min}$, then methylmagnesiumchloride in THF (c = 3.26 M, $32 \mu \mathrm{L}, 0.10 \mathrm{mmol}, 2.0$ equiv.) was added. After shaking 
the vial at $23{ }^{\circ} \mathrm{C}$ for 30 seconds to dissolve frozen droplets of the Grignard reagent, the vial was chilled at $-35{ }^{\circ} \mathrm{C}$ for 20 hours in a freezer. Three more portions of methylmagnesium chloride in THF $(\mathrm{c}=3.26 \mathrm{M}$, each: $16 \mu \mathrm{L}, 51 . \mu \mathrm{mol}, 1.0$ equiv.) were added at $-45^{\circ} \mathrm{C}$. Each addition was followed by 24 hours at -35 ${ }^{\circ} \mathrm{C}$. The reaction vial was warmed at $23{ }^{\circ} \mathrm{C}$ for $30 \mathrm{~min}$, at which point a white solid had precipitated. Filtration through glass wool and concentration of the filtrate under reduced pressure afforded a solid, which was triturated with $\mathrm{Et}_{2} \mathrm{O}(0.5 \mathrm{~mL})$ and dried in vacuo to afford the title compound $(25.4 \mathrm{mg}, 75 \%)$. NMR Spectroscopy: ${ }^{1} \mathrm{H}$ NMR (400 MHz, $\left.\mathrm{CD}_{2} \mathrm{Cl}_{2}, 23{ }^{\circ} \mathrm{C}, \delta\right): 7.61(\mathrm{t}, J=6.2 \mathrm{~Hz}, 1 \mathrm{H}), 7.36(\mathrm{~d}, J=7.4 \mathrm{~Hz}$, $1 \mathrm{H}), 7.18(\mathrm{t}, J=7.8 \mathrm{~Hz}, 1 \mathrm{H}), 7.02(\mathrm{t}, J=7.4 \mathrm{~Hz}, 1 \mathrm{H}), 3.98(\mathrm{~s}, \mathrm{br}, 24 \mathrm{H}), 3.00\left(\mathrm{~s},{ }^{2} J_{\mathrm{Pt}-\mathrm{H}}=100 \mathrm{~Hz}, 2 \mathrm{H}\right), 2.21$ $\left(\mathrm{s},{ }^{2} J_{\mathrm{Pt}-\mathrm{H}}=7.8 \mathrm{~Hz}, 3 \mathrm{H}\right), 1.93(\mathrm{~s}, \mathrm{br}, 24 \mathrm{H}), 1.33\left(\mathrm{~d},{ }^{3} J_{\mathrm{P}-\mathrm{H}}=12.5 \mathrm{~Hz}, 18 \mathrm{H}\right), 0.26\left(\mathrm{~d},{ }^{2} J_{\mathrm{Pt}-\mathrm{H}}=67 \mathrm{~Hz},{ }^{3} J_{\mathrm{P}-\mathrm{H}}=\right.$ $7.4 \mathrm{~Hz}, 3 \mathrm{H}) .{ }^{31} \mathrm{P}$ NMR $\left(162 \mathrm{MHz}, \mathrm{CD}_{2} \mathrm{Cl}_{2}, 23{ }^{\circ} \mathrm{C}, \delta\right): 74.51\left({ }^{1} \mathrm{~J}_{\mathrm{P}-\mathrm{Pt}}=2406 \mathrm{~Hz}\right)$.

\section{$\left[\kappa^{2}-(o-\mathrm{Tol})_{2} \mathrm{P}\left(\mathrm{C}_{6} \mathrm{H}_{4}-\text { ortho-CH2)PtCl}\right]_{2}(49)\right.$}

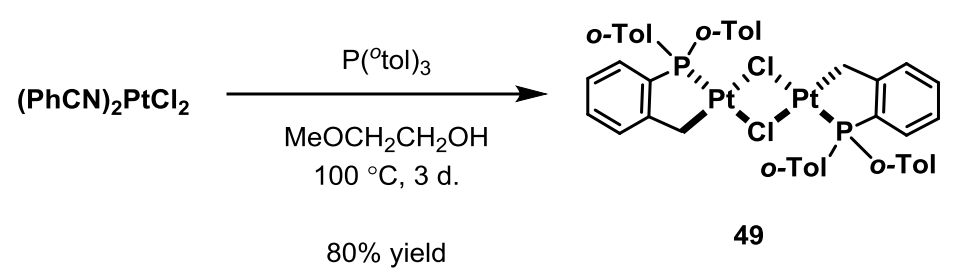

Complex 49 was synthesized by the method of Ferrer et al. ${ }^{13}$ Under ambient atmosphere, $(\mathrm{PhCN})_{2} \mathrm{PtCl}_{2}$ (81.1 mg, 0.172 mmol, 1.00 equiv.), $\mathrm{P}(o-T o l)_{3}(52.3 \mathrm{mg}, 0.172 \mathrm{mmol}, 1.00$ equiv.), degassed 2methoxyethanol $(1.0 \mathrm{~mL})$, and a Teflon-coated magnetic stirring bar were added to a $4 \mathrm{~mL}$ glass scintillation vial. The atmosphere of the vial was purged with dry $\mathrm{N}_{2}$, then the vial was sealed with a Teflon-lined cap and heated at $100{ }^{\circ} \mathrm{C}$ in an aluminum heating block for 2 hours. DIPEA (30 $\left.\mu \mathrm{L}\right)$ was added and the mixture turned black. The precipitate was collected by centrifugation and decantation of the solvent and washed with EtOH $(2 \times 1 \mathrm{~mL})$ and hexane $(2 \times 1 \mathrm{~mL})$, then dried in vacuo to afford the title

\footnotetext{
${ }^{13}$ Ferrer, C.; Raducan, M.; Nevado, C.; Claverie, C. K.; Echavarren, A. M. Tetrahedron 2007, 63, 6306.
} 
compound as a white solid (73.7 mg, 80\%). Due to insolubility in common organic solvents, this material was taken forward in the synthesis of $\mathbf{5 0}$ without spectroscopic characterization.

\section{$\left[\kappa^{2}-(o-\mathrm{Tol})_{2} \mathrm{P}\left(\mathrm{C}_{6} \mathrm{H}_{4}-\right.\right.$ ortho-CH$\left.) \mathrm{Pt}(\mathrm{NCMe})_{2}\right][\mathrm{OTf}](50)$}

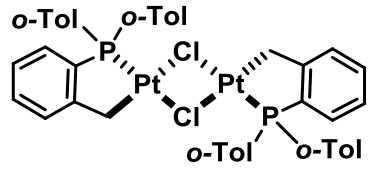

49

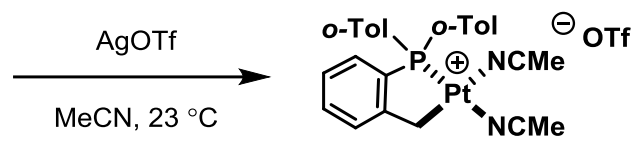

$78 \%$ yield

50

The method for synthesizing $\mathbf{5 0}$ was adapted from that of Ferrer et al. ${ }^{13}$ In a dry, $\mathrm{N}_{2}$-filled glovebox, platinum complex 49 (68.4 mg, $69.0 \mu \mathrm{mol}, 1.00$ equiv.), AgOTf (32.9 mg, $128 \mu \mathrm{mol}, 2.00$ equiv.), MeCN $(1.5 \mathrm{~mL})$, and a Teflon-coated stirring bar were added to a $20 \mathrm{~mL}$ scintillation vial. The suspension was stirred for 12 hours at $23{ }^{\circ} \mathrm{C}$, then DCM $(1.0 \mathrm{~mL})$ was added and the white suspension was filtered through a plug of glass wool. The colorless filtrate was concentrated under reduced pressure and the resulting colorless gel was triturated with $\mathrm{Et}_{2} \mathrm{O}$ and dried in vacuo to afford the title compound as a colorless solid (76.6 mg, 78\%).

NMR Spectroscopy: ${ }^{1} \mathrm{H}$ NMR (400 MHz, $\left.\mathrm{CD}_{2} \mathrm{Cl}_{2}, 23{ }^{\circ} \mathrm{C}, \delta\right): 7.55-6.90(\mathrm{~m}, 12 \mathrm{H}), 3.45\left(\mathrm{~s},{ }^{2} \mathrm{Jt}_{\mathrm{Pt}-\mathrm{H}}=99 \mathrm{~Hz}\right)$, $2.61(\mathrm{~s}, \mathrm{br}, 3 \mathrm{H}), 2.52(\mathrm{~s}, \mathrm{br}, 3 \mathrm{H}), 2.15(\mathrm{~s}, 6 \mathrm{H}) .{ }^{31} \mathrm{P} \mathrm{NMR}\left(162 \mathrm{MHz}, \mathrm{CD}_{2} \mathrm{Cl}_{2}, 23{ }^{\circ} \mathrm{C}, \delta\right): 17.0\left({ }^{1} J_{\mathrm{P}-\mathrm{Pt}}=4673\right.$ $\mathrm{Hz})$.

$\left[\kappa^{2}-(o-\mathrm{Tol})_{2} \mathrm{P}\left(\mathrm{C}_{6} \mathrm{H}_{4}-\right.\right.$ ortho-CH$\left.) \mathrm{PtMe}_{2}\right][\mathrm{MgCl}](39)$

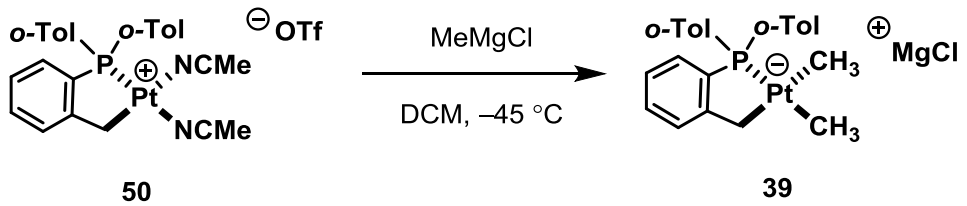

In a dry, $\mathrm{N}_{2}$-filled glovebox, platinum complex $50(76.6 \mathrm{mg}, 105 \mu \mathrm{mol}, 1.00$ equiv.), toluene (1.0 mL), and a Teflon-coated magnetic stirring bar were added to a $4 \mathrm{~mL}$ glass scintillation vial at $23{ }^{\circ} \mathrm{C}$. The vial was chilled at $-45{ }^{\circ} \mathrm{C}$ in a $\mathrm{CO}_{2} /{ }^{i} \mathrm{PrOH}-$ cooled cold well for $20 \mathrm{~min}$., then methylmagnesiumchloride in 
THF (c = $3.25 \mathrm{M}, 65 \mu \mathrm{L}, 0.21 \mathrm{mmol}, 2.0$ equiv.) was added. After shaking the vial at $23{ }^{\circ} \mathrm{C}$ for 30 seconds to dissolve frozen droplets of the Grignard reagent, the vial was chilled at $-35^{\circ} \mathrm{C}$ for 24 hours in a freezer. The reaction vial was warmed at $23{ }^{\circ} \mathrm{C}$ for $30 \mathrm{~min}$, at which point a white solid had precipitated. Filtration through glass wool and concentration of the filtrate under reduced pressure afforded a solid, which was triturated with $\mathrm{Et}_{2} \mathrm{O}(0.5 \mathrm{~mL})$ and dried in vacuo to afford the title compound.

NMR Spectroscopy: ${ }^{1} \mathrm{H}$ NMR (400 MHz, $\mathrm{CD}_{2} \mathrm{Cl}_{2}, 23{ }^{\circ} \mathrm{C}, \delta$ ): $7.50-6.65$ (m, 12H), 3.88 (s, br, 8H), 2.69 (s, br, 2H), $2.40(\mathrm{~s}, 6 \mathrm{H}), 1.85(\mathrm{~s}, \mathrm{br}, 8 \mathrm{H}), 0.70\left(\mathrm{~s}, \mathrm{br},{ }^{2} \mathrm{Jt}_{\mathrm{Pt}-\mathrm{H}}=42 \mathrm{~Hz}, 3 \mathrm{H}\right), 0.22\left(\mathrm{~s}\right.$, br, $\left.{ }^{2} J_{\mathrm{Pt}-\mathrm{H}}=35 \mathrm{~Hz}, 3 \mathrm{H}\right)$. ${ }^{31} \mathrm{P}$ NMR $\left(162 \mathrm{MHz}, \mathrm{CD}_{2} \mathrm{Cl}_{2}, 23{ }^{\circ} \mathrm{C}, \delta\right): 37.1\left({ }^{1} J_{\mathrm{P}-\mathrm{Pt}}=2254 \mathrm{~Hz}\right)$.

\section{$\left.\left[\left(\kappa^{2}-\left({ }^{t} \mathrm{Bu}\right)_{2} \mathrm{PCMe}_{2} \mathrm{CH}_{2}\right)\right) \mathrm{PtBn}_{2}\right][\mathrm{K}](51)$}
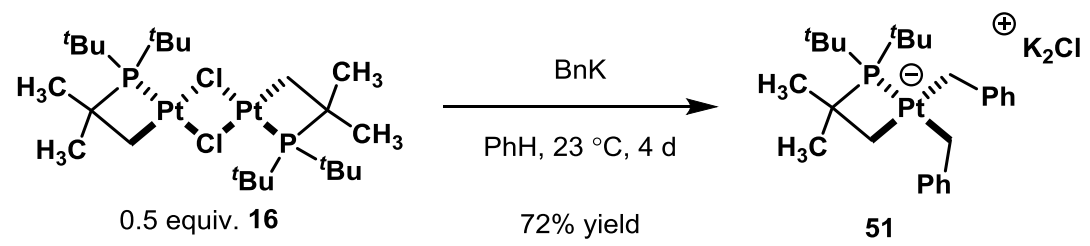

In a dry, $\mathrm{N}_{2}$-filled glovebox, Platinum dimer 16 (10.0 mg, $12.0 \mu \mathrm{mol}, 1.00$ equiv.), benzylpotassium (6.0 $\mathrm{mg}, 46.0 \mu \mathrm{mol}, 4.00$ equiv.), benzene ( $1 \mathrm{~mL})$, and a Teflon-coated magnetic stirring bar were added to a 4 $\mathrm{mL}$ glass scintillation vial. The resulting yellow solution was stirred at $23{ }^{\circ} \mathrm{C}$ for 4 days, then filtered through a plug of glass wool and concentrated under reduced pressure. The resulting yellow solid was recrystallized from benzene/hexanes to yield the title compound as a yellow crystalline solid (10.2 mg, $72 \%)$.

NMR Spectroscopy: ${ }^{1} \mathrm{H}$ NMR (400 MHz, $\left.\mathrm{C}_{6} \mathrm{D}_{6}, 23{ }^{\circ} \mathrm{C}, \delta\right): 7.25(\mathrm{~m}, 4 \mathrm{H}), 7.04(\mathrm{t}, J=7.4 \mathrm{~Hz}, 2 \mathrm{H}), 6.99$ (t, $J=7.4 \mathrm{~Hz}, 2 \mathrm{H}), 6.66(\mathrm{t}, J=6.8 \mathrm{~Hz}, 1 \mathrm{H}), 6.57(\mathrm{t}, J=7.0 \mathrm{~Hz}, 1 \mathrm{H}), 2.97\left(\mathrm{~d},{ }^{3} J_{\mathrm{P}-\mathrm{H}}=3.5 \mathrm{~Hz},{ }^{2} J_{\mathrm{Pt}-\mathrm{H}}=80.0 \mathrm{~Hz}\right.$, $2 \mathrm{H}), 2.69\left(\mathrm{~d},{ }^{3} J_{\mathrm{P}-\mathrm{H}}=10.5 \mathrm{~Hz},{ }^{2} J_{\mathrm{Pt}-\mathrm{H}}=97.1 \mathrm{~Hz}, 2 \mathrm{H}\right), 1.44(\mathrm{~d}, J=12.1 \mathrm{~Hz}, 6 \mathrm{H}), 1.39(\mathrm{~d}, J=10.9 \mathrm{~Hz}, 18 \mathrm{H})$, $0.97(\mathrm{~d}, J=13.3 \mathrm{~Hz}, 2 \mathrm{H}) .{ }^{31} \mathrm{P} \mathrm{NMR}\left(162 \mathrm{MHz}, \mathrm{CDCl}_{3}, 23{ }^{\circ} \mathrm{C}, \delta\right): 8.3\left({ }^{1} J_{\mathrm{P}-\mathrm{Pt}}=1738 \mathrm{~Hz}\right)$. 
$\left.\left[\left(\kappa^{2}-\left({ }^{t} \mathrm{Bu}\right)_{2} \mathrm{PCMe} \mathrm{CH}_{2}\right)\right) \operatorname{Pt}\left(\mathrm{CH}_{2} \mathrm{CH}_{2} \mathrm{CH}=\mathrm{CH}_{2}\right)_{2}\right][\mathrm{Li}](43)$

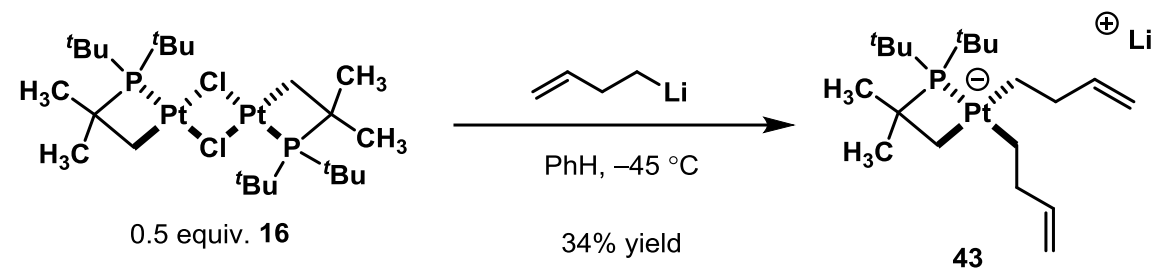

In a dry, $\mathrm{N}_{2}$-filled glovebox, platinum complex 16 (100. mg, $116 \mathrm{mmol}, 1.00$ equiv.), benzene (5.0 mL), and a Teflon-coated magnetic stirring bar were added to a $20 \mathrm{~mL}$ glass scintillation vial. The vial was cooled at $-45{ }^{\circ} \mathrm{C}$ for $30 \mathrm{~min}$. and butenyllithium in hexanes $(\mathrm{c}=0.26 \mathrm{M}, 2.3 \mathrm{~mL}, 0.58 \mathrm{mmol}, 5.0$ equiv.) was added. The vial was warmed at $23{ }^{\circ} \mathrm{C}$ for 2 hours, then chilled at $-45^{\circ} \mathrm{C}$. Triethoxysilane $(50, \mu \mathrm{L}, 45$ $\mathrm{mg}, 0.27 \mathrm{mmol}, 2.3$ equiv.) was added and the vial was warmed at $23{ }^{\circ} \mathrm{C}$. The resulting yellow suspension was filtered through a plug of glass wool, frozen at $-45^{\circ} \mathrm{C}$, and the frozen solution was lyophilized under reduced pressure to afford a gelatinous yellow solid. Trituration with hexanes $(2 \mathrm{~mL})$ afforded the product as an off-white solid (20.0 $\mathrm{mg}, 34 \%)$.

NMR Spectroscopy: ${ }^{1} \mathrm{H}$ NMR (400 MHz, $\left.\mathrm{CD}_{2} \mathrm{Cl}_{2}, 23{ }^{\circ} \mathrm{C}, \delta\right): 6.43$ (s, br) 6.16 (s, br), 5.1 (m, br), 4.9 (m, br), 2.6 (s, br). Other peaks obscured by solvent. ${ }^{31} \mathrm{P} \mathrm{NMR}\left(162 \mathrm{MHz}, \mathrm{CD}_{2} \mathrm{Cl}_{2}, 23{ }^{\circ} \mathrm{C}, \delta\right): 10.7\left({ }^{1} J_{\mathrm{P}-\mathrm{Pt}}=\right.$ $1601 \mathrm{~Hz})$.
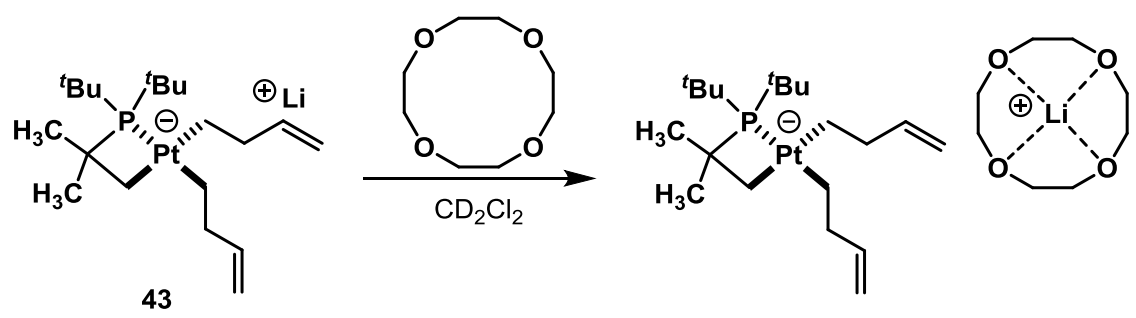

Addition of Lewis basic molecules that bind to $\mathrm{Li}^{+}$sharpened the ${ }^{1} \mathrm{H}$ NMR spectrum of the title compound and these spectral data are reported for addition of 12-crown-8: ${ }^{1} \mathrm{H} \mathrm{NMR}\left(400 \mathrm{MHz}, \mathrm{CD}_{2} \mathrm{Cl}_{2}\right.$, $\left.23{ }^{\circ} \mathrm{C}, \delta\right): 6.6(\mathrm{~m}, 1 \mathrm{H}), 5.26(\mathrm{~d}, J=16.8 \mathrm{~Hz}, 1 \mathrm{H}), 5.0(\mathrm{~d}, J=11.7 \mathrm{~Hz}, 1 \mathrm{H}), 3.52(\mathrm{t}, J=13.7 \mathrm{~Hz}, 2 \mathrm{H}), 3.3$ 
(s, 30H), 2.9 (m, br, 2H), $2.2(\mathrm{~d}, J=2.7 \mathrm{~Hz}, 2 \mathrm{H}), 2.1$ (d, $J=6.2 \mathrm{~Hz}, 4 \mathrm{H}), 1.8(\mathrm{~d}, J=11.3 \mathrm{~Hz}, 6 \mathrm{H}), 1.7$ (d, $J=10.5 \mathrm{~Hz}, 18 \mathrm{H}), 1.45-1.10(\mathrm{~m}, 8 \mathrm{H}), 0.95-0.78(\mathrm{~m}, 4 \mathrm{H})$.

\section{$\left[\left(\kappa^{2}-\left({ }^{t} \mathrm{Bu}\right)_{2} \mathrm{PCMe}_{2} \mathrm{CH}_{2}\right) \operatorname{Pt}(\mathrm{py})_{2}\right][\mathrm{OTf}](52)$}

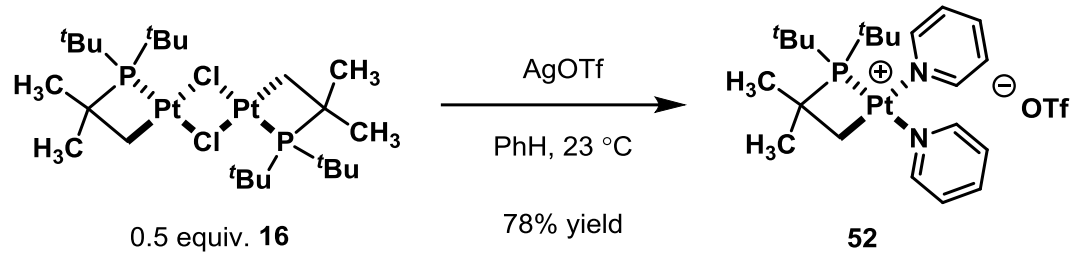

In a dry, $\mathrm{N}_{2}$-filled glovebox, platinum dimer 16 (0.100 g, $0.116 \mathrm{mmol}, 1.00$ equiv.), benzene ( $\left.3 \mathrm{~mL}\right)$, and a Teflon-coated stirring bar were added to a $20 \mathrm{~mL}$ glass scintillation vial at $23{ }^{\circ} \mathrm{C}$. A separate vial was charged with AgOTf (59.5 mg, $0.232 \mathrm{mmol}, 2.00$ equiv.) and acetonitrile (1 mL), forming a clear, colorless solution. The solution of AgOTf was added to the solution containing platinum dimer $\mathbf{1 6}$ while stirring, at which point a white solid precipitated. Pyridine (37 $\mu \mathrm{L}, 36.6 \mathrm{mg}, 0.463 \mathrm{mmol}, 4.0$ equiv.) was added and the white suspension was stirred for $5 \mathrm{~min}$. before it was filtered through glass wool. The clear, colorless filtrate was concentrated under reduced pressure to afford the title compound as a colorless solid $(0.128 \mathrm{~g}, 78 \%)$.

NMR Spectroscopy: ${ }^{1} \mathrm{H}$ NMR (400 MHz, $\left.\mathrm{CD}_{2} \mathrm{Cl}_{2}, 23{ }^{\circ} \mathrm{C}, \delta\right): 8.77(\mathrm{~d}, J=6.3 \mathrm{~Hz}, 2 \mathrm{H}), 8.45(\mathrm{~m}, 2 \mathrm{H}), 7.89$ $(\mathrm{t}, J=8.3 \mathrm{~Hz}, 2 \mathrm{H}), 7.52(\mathrm{t}, 7.6 \mathrm{~Hz}, 2 \mathrm{H}), 7.51(\mathrm{t}, J=6.6 \mathrm{~Hz}, 2 \mathrm{H}), 1.57(\mathrm{~d}, J=14.2 \mathrm{~Hz}, 6 \mathrm{H}), 1.46(\mathrm{~m}, 20 \mathrm{H})$. ${ }^{31} \mathrm{P}$ NMR $\left(162 \mathrm{MHz}, \mathrm{CD}_{2} \mathrm{Cl}_{2}, 23{ }^{\circ} \mathrm{C}, \delta\right):-13.8\left({ }^{1} \mathrm{~J}_{\mathrm{P}-\mathrm{Pt}}=3125 \mathrm{~Hz}\right) \cdot{ }^{19} \mathrm{~F}$ NMR: $\left(376 \mathrm{MHz}, \mathrm{CD}_{2} \mathrm{Cl}_{2}, 23{ }^{\circ} \mathrm{C}\right.$, $\delta):-78.9$.

\section{$\left(\kappa^{2}-\left({ }^{t} \mathrm{Bu}\right)_{2} \mathrm{PCMe}_{2} \mathrm{CH}_{2}\right) \operatorname{Pt}\left(\mathrm{CH}_{3}\right)(\mathrm{py})(53)$}

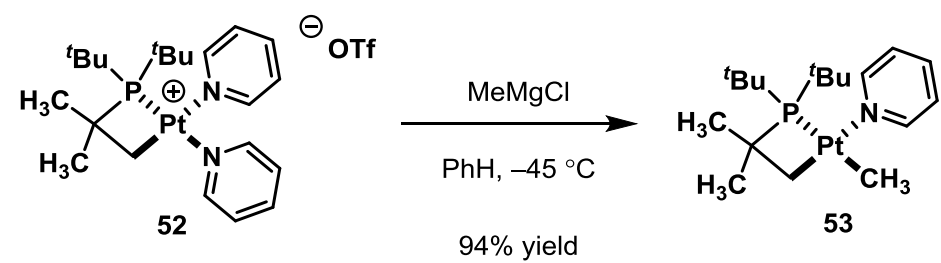


In a dry, $\mathrm{N}_{2}$-filled glovebox, platinum complex 52 (204 mg, $0.290 \mathrm{mmol}, 1.00$ equiv.), benzene (7 mL), and a Teflon-coated magnetic stirring bar were added to a $20 \mathrm{~mL}$ glass scintillation vial. The vial was chilled at $-45^{\circ} \mathrm{C}$ for $15 \mathrm{~min}$., then $\mathrm{MeMgCl}$ in THF (c = 3.25 M, $0.290 \mathrm{~mL}, 0.290 \mathrm{mmol}, 1.00$ equiv.) was added and the vial was warmed at $23{ }^{\circ} \mathrm{C}$ for $30 \mathrm{~min}$. The white suspension was filtered through celite and concentrated under reduced pressure to yield the title compound as a colorless solid (133.5 $\mathrm{mg}, 94 \%)$. NMR Spectroscopy: ${ }^{1} \mathrm{H}$ NMR $\left(400 \mathrm{MHz}, \mathrm{C}_{6} \mathrm{D}_{6}, 23{ }^{\circ} \mathrm{C}, \delta\right): 8.82(\mathrm{~d}, J=6.2 \mathrm{~Hz}, 2 \mathrm{H}), 6.67$ (t, $J=7.8 \mathrm{~Hz}$, $1 \mathrm{H}), 6.34(\mathrm{~m}, 2 \mathrm{H}), 2.01\left(\mathrm{~d},{ }^{2} J_{\mathrm{Pt}-\mathrm{H}}=92.4 \mathrm{~Hz},{ }^{3} J_{\mathrm{P}-\mathrm{H}}=15.2 \mathrm{~Hz}, 2 \mathrm{H}\right), 1.62(\mathrm{~d}, J=12.0 \mathrm{~Hz}, 6 \mathrm{H}), 1.35(\mathrm{~d}, 11.3$ $\mathrm{Hz}, 18 \mathrm{H}), 1.08\left(\mathrm{~d},{ }^{2} J_{\mathrm{Pt}-\mathrm{H}}=71.4 \mathrm{~Hz},{ }^{3} J_{\mathrm{P}-\mathrm{H}}=7.4 \mathrm{~Hz}, 3 \mathrm{H}\right) .{ }^{31} \mathrm{P} \mathrm{NMR}\left(162 \mathrm{MHz}, \mathrm{CD}_{2} \mathrm{Cl}_{2}, 23{ }^{\circ} \mathrm{C}, \delta\right): 26.4\left({ }^{1} J_{\mathrm{P}-}\right.$ $\mathrm{Pt}=1274 \mathrm{~Hz})$.

\section{$\left(\kappa^{2}-\left({ }^{t} \mathrm{Bu}\right)_{2} \mathrm{PCMe}_{2} \mathrm{CH}_{2}\right) \operatorname{Pt}\left(\mathrm{Si}(\mathrm{OEt})_{3}\right)(\mathrm{py})(54)$}
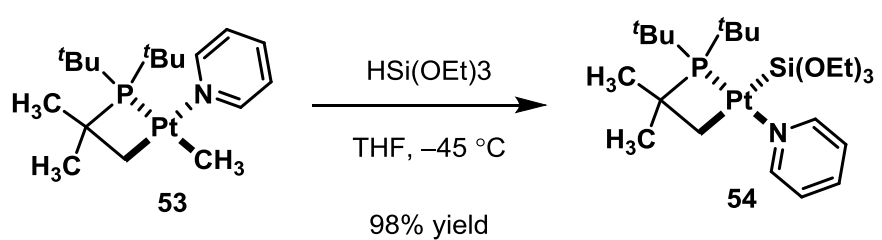

In a dry, $\mathrm{N}_{2}$-filled glovebox, platinum complex 53 (359 mg, $0.732 \mathrm{mmol}, 1.00$ equiv.), THF (0.5 mL), and a Teflon-coated magnetic stirring bar were added to a $20 \mathrm{~mL}$ glass scintillation vial. The vial was chilled at $-45{ }^{\circ} \mathrm{C}$ for $15 \mathrm{~min}$. and triethoxysilane (135 $\mu \mathrm{L}, 120 . \mathrm{mg}, 0.732 \mathrm{mmol}, 1.00$ equiv.) was added. The vial was warmed at $23{ }^{\circ} \mathrm{C}$ for 2 hours, then the clear colorless solution was concentrated under reduced pressure to yield the title compound as a white solid (459. $\mathrm{mg}, 98 \%$ ).

NMR Spectroscopy: ${ }^{1} \mathrm{H}$ NMR (400 MHz, $\left.\mathrm{C}_{6} \mathrm{D}_{6}, 23{ }^{\circ} \mathrm{C}, \delta\right): 8.99(\mathrm{~m}, 2 \mathrm{H}), 6.66(\mathrm{t}, J=7.8 \mathrm{~Hz}, 1 \mathrm{H}), 6.40(\mathrm{t}$, $J=6.4 \mathrm{~Hz}, 2 \mathrm{H}), 4.05(\mathrm{q}, J=7.0 \mathrm{~Hz}, 6 \mathrm{H}), 3.58(\mathrm{t}, J=6.6 \mathrm{~Hz}, 4 \mathrm{H}), 1.61(\mathrm{~d}, J=12.5 \mathrm{~Hz}, 18 \mathrm{H}), 1.56(\mathrm{~d}, J=$ $13.3 \mathrm{~Hz}, 6 \mathrm{H}), 1.28(\mathrm{t}, J=7.0 \mathrm{~Hz}, 9 \mathrm{H}), 0.83\left(\mathrm{~d},{ }^{2} J_{\mathrm{Pt}-\mathrm{H}}=48.4 \mathrm{~Hz},{ }^{3} J_{\mathrm{P}-\mathrm{H}}=10.1 \mathrm{~Hz}, 2 \mathrm{H}\right) .{ }^{31} \mathrm{P}$ NMR $(162$ $\left.\mathrm{MHz}, \mathrm{CD}_{2} \mathrm{Cl}_{2}, 23{ }^{\circ} \mathrm{C}, \delta\right):-5.28\left({ }^{1} J_{\mathrm{P}-\mathrm{Pt}}=3171 \mathrm{~Hz}\right)$. 


\section{$\left[\left(\kappa^{2}-\left({ }^{t} \mathrm{Bu}\right)_{2} \mathrm{PCMe} 2 \mathrm{CH}_{2}\right) \operatorname{Pt}\left(\mathrm{Si}(\mathrm{OEt}){ }_{3}\right)\left(\mathrm{CH}_{2} \mathrm{CH}_{2} \mathrm{CH}=\mathrm{CH}_{2}\right)\right][\mathrm{MgCl}](34)$}

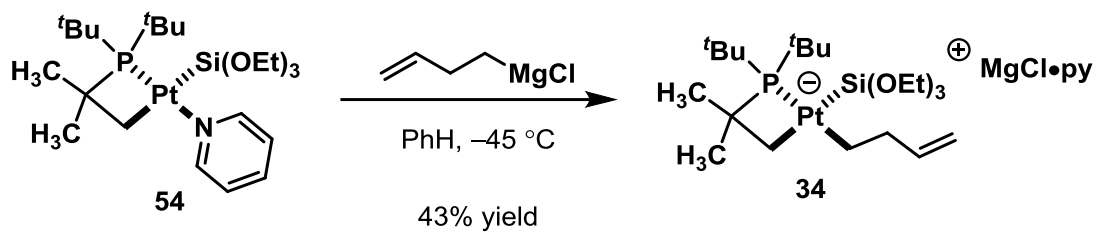

In a dry, $\mathrm{N}_{2}$-filled glovebox, platinum complex 54 (459. $\mathrm{mg}, 0.719 \mathrm{mmol}, 1.00$ equiv.), benzene (5 mL), and a Teflon-coated magnetic stirring bar were added to a $20 \mathrm{~mL}$ glass scintillation vial. The vial was chilled at $-45^{\circ} \mathrm{C}$ for $30 \mathrm{~min}$., then butenylmagnesium chloride in THF (c $=1.15 \mathrm{M}, 625 \mu \mathrm{L}, 0.719 \mathrm{mmol}$, 1.00 equiv.) was added and the vial was warmed at $23{ }^{\circ} \mathrm{C}$ for $30 \mathrm{~min}$. The clear red solution was frozen, then lyophilized under reduced pressure. The residue was triturated with pentane $(10 \mathrm{~mL})$ to afford the title compound as a white solid (542 mg., 43\%).

NMR Spectroscopy: ${ }^{1} \mathrm{H}$ NMR (400 MHz, $\left.\mathrm{C}_{6} \mathrm{D}_{6}, 23{ }^{\circ} \mathrm{C}, \delta\right): 9.0-8.6$ (s, br., 2H), 6.89-6.71 (s, br, 1H), 6.65-6.49 (s, br, 2H), 6.33-6.05 (m, br, 1H), 5.08-4.74 (m, 2H), 4.07-3.86 (m, br, 6H), 3.75-3.59 (s, br, 4H), 3.0-2.74 (m, br, 2H), 1.68 (d, $J=10.9 \mathrm{~Hz}, 6 \mathrm{H}), 1.56(\mathrm{~d}, J=10.5 \mathrm{~Hz}, 18 \mathrm{H}), 1.45-1.10$ (m), $0.95-$ 0.75 (m). ${ }^{31} \mathrm{P}$ NMR $\left(162 \mathrm{MHz}, \mathrm{CD}_{2} \mathrm{Cl}_{2}, 23{ }^{\circ} \mathrm{C}, \delta\right): 14.0\left({ }^{1} J_{\mathrm{P}-\mathrm{Pt}}=1262 \mathrm{~Hz}\right)$.

\section{$\left[\left(\kappa^{2}-\left({ }^{t} \mathrm{Bu}\right)_{2} \mathrm{PCMe} \mathrm{CH}_{2}\right) \mathrm{Pt}\left(\mathrm{Si}(\mathrm{OEt})_{3}\right)\left(\mathrm{CH}_{2} \mathrm{CH}_{2} \mathrm{CH}=\mathrm{CH}_{2}\right)\right]\left[\mathrm{NEt}_{4}\right](33)$}

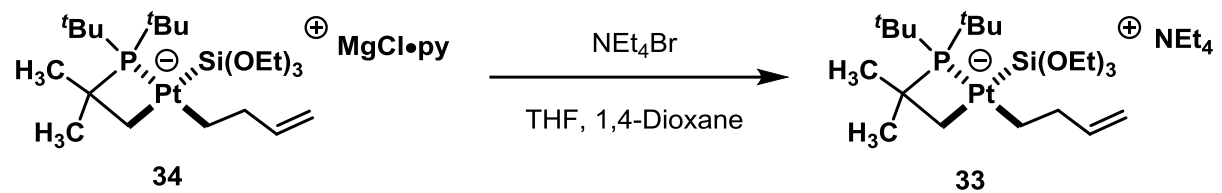

In a dry, $\mathrm{N}_{2}$-filled glovebox, platinum complex $34(10.0 \mathrm{mg}, 13.0 \mu \mathrm{mol}, 1.00$ equiv. $)$, tetrabutylammonium bromide (4.3 mg, $13 \mu \mathrm{mol}, 1.00$ equiv.), THF (0.5 mL), and a Teflon-coated magnetic stirring bar were added to a $4 \mathrm{~mL}$ glass scintillation vial to form a clear, colorless solution. Dioxane $(1.0 \mathrm{~mL})$ was added and the resulting white suspension was stirred at $23{ }^{\circ} \mathrm{C}$ for 12 hours, then 
filtered. The clear, colorless filtrate was concentrated under reduced pressure to afford the title compound as an orange residue. The title compound was used without further isolation or purification.

NMR Spectroscopy: ${ }^{1} \mathrm{H}$ NMR (400 MHz, $\left.\mathrm{C}_{6} \mathrm{D}_{6}, 23{ }^{\circ} \mathrm{C}, \delta\right): 6.64(\mathrm{~m}, 1 \mathrm{H}), 5.28(\mathrm{~d}, J=17.1 \mathrm{~Hz}, 1 \mathrm{H}) .5 .0(\mathrm{~d}$, $J=8.3 \mathrm{~Hz}, 1 \mathrm{H}), 4.5-4.3(\mathrm{~m}, 14 \mathrm{H}), 3.08(\mathrm{~s}, 8 \mathrm{H}), 2.10-2.25(\mathrm{~m}, 2 \mathrm{H}), 1.78(\mathrm{~d}, J=12.7 \mathrm{~Hz}, 6 \mathrm{H}), 1.68(\mathrm{~d}, J=$ $10.2 \mathrm{~Hz}, 18 \mathrm{H}), 1.53(\mathrm{t}, J=6.8 \mathrm{~Hz}, 9 \mathrm{H}), 1.45-1.25(\mathrm{~m}, 16 \mathrm{H}), 0.96(\mathrm{t}, J=7.8 \mathrm{~Hz}, 12 \mathrm{H}) .{ }^{31} \mathrm{P}$ NMR $(162$ $\left.\mathrm{MHz}, \mathrm{CD}_{2} \mathrm{Cl}_{2}, 23{ }^{\circ} \mathrm{C}, \delta\right): 11.3\left({ }^{1} J_{\mathrm{P}-\mathrm{Pt}}=1243 \mathrm{~Hz}\right)$.

$\left[\left(\kappa^{2}-\left({ }^{t} \mathrm{Bu}_{2} \mathrm{PCMe}_{2} \mathrm{CH}_{2}\right) \operatorname{Pt}\left(\mathrm{SMe}_{2}\right)_{2}\right][\mathrm{OTf}](55)\right.$

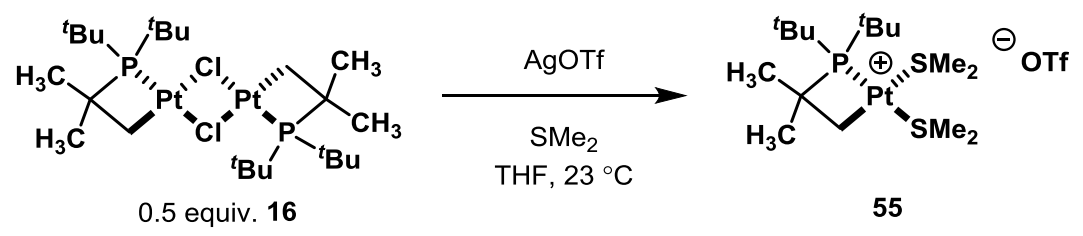

Under ambient atmosphere, platinum dimer 16 (320.4 mg, $0.371 \mathrm{mmol}, 1.00$ equiv.), THF (3 mL), and a Teflon-coated stirring bar were added to a $20 \mathrm{~mL}$ glass scintillation vial at $23{ }^{\circ} \mathrm{C}$. A separate vial was charged with AgOTf (191 mg, $0.742 \mathrm{mmol}, 2.00$ equiv.) and THF (1 mL), forming a clear, colorless solution. The solution of AgOTf was added to the solution containing platinum dimer $\mathbf{1 6}$ while stirring, at which point a white solid precipitated. The suspension was filtered through glass wool to afford a clear, colorless filtrate. Dimethylsulfide (272 $\mu \mathrm{L}, 230 \mathrm{mg}, 3.71 \mathrm{mmol}, 10.0$ equiv.) was added and the colorless solution was stirred for $5 \mathrm{~min}$. and concentrated under reduced pressure to afford the title compound as a colorless solid.

NMR Spectroscopy: ${ }^{1} \mathrm{H}$ NMR (400 MHz, $\left.\mathrm{C}_{6} \mathrm{D}_{6}, 23{ }^{\circ} \mathrm{C}, \delta\right): 2.48-2.32(\mathrm{~m}, 12 \mathrm{H}), 1.45-1.32(\mathrm{~m}, 26 \mathrm{H}) .{ }^{31} \mathrm{P}$ $\operatorname{NMR}\left(162 \mathrm{MHz}, \mathrm{CD}_{2} \mathrm{Cl}_{2}, 23{ }^{\circ} \mathrm{C}, \delta\right):-14.6\left({ }^{1} \mathrm{JP}_{\mathrm{Pt}}=2816 \mathrm{~Hz}\right)$. 
$\left(\kappa^{2}-\left({ }^{t} \mathrm{Bu}\right)_{2} \mathrm{PCMe}_{2} \mathrm{CH}_{2}\right) \mathrm{Pt}\left(\mathrm{CH}_{3}\right)\left(\mathrm{SMe}_{2}\right)(56)$

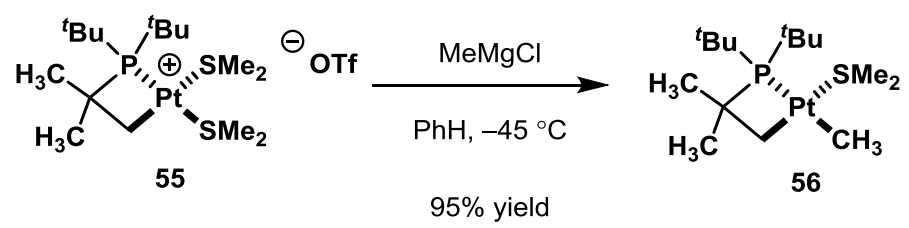

In a dry, $\mathrm{N}_{2}$-filled glovebox, platinum complex 55 (250.0 mg, $0.373 \mathrm{mmol}, 1.00$ equiv.), benzene (6 mL), and a Teflon-coated magnetic stirring bar were added to a $20 \mathrm{~mL}$ glass scintillation vial. The vial was chilled at $-45{ }^{\circ} \mathrm{C}$ for $15 \mathrm{~min}$., then $\mathrm{MeMgCl}$ in $\mathrm{THF}(\mathrm{c}=3.25 \mathrm{M}, 115 \mu \mathrm{L}, 0.373 \mathrm{mmol}, 1.00$ equiv.) was added and the vial was warmed at $23{ }^{\circ} \mathrm{C}$ for $30 \mathrm{~min}$. The white suspension was filtered through celite and concentrated under reduced pressure to yield the title compound as a colorless solid (177 mg, 95\%).

NMR Spectroscopy: ${ }^{1} \mathrm{H}$ NMR $\left(400 \mathrm{MHz}, \mathrm{C}_{6} \mathrm{D}_{6}, 23{ }^{\circ} \mathrm{C}, \delta\right): 2.01\left(\mathrm{~s},{ }^{3} J_{\mathrm{Pt}-\mathrm{H}}=24.2 \mathrm{~Hz}, 6 \mathrm{H}\right), 1.80\left(\mathrm{~d},{ }^{2} J_{\mathrm{Pt}-\mathrm{H}}=\right.$ $\left.90.5 \mathrm{~Hz},{ }^{3} J_{\mathrm{P}-\mathrm{H}}=14.4 \mathrm{~Hz}, 2 \mathrm{H}\right), 1.50(\mathrm{~d}, J=12 \mathrm{~Hz}, 6 \mathrm{H}), 1.35(\mathrm{~d}, J=11.3,18 \mathrm{H}), 0.98\left(\mathrm{~d},{ }^{2} J_{\mathrm{Pt}-\mathrm{H}}=70.6 \mathrm{~Hz}\right.$, $\left.{ }^{3} J_{\mathrm{P}-\mathrm{H}}=7.4 \mathrm{~Hz}, 3 \mathrm{H}\right) .{ }^{31} \mathrm{P} \mathrm{NMR}\left(162 \mathrm{MHz}, \mathrm{CD}_{2} \mathrm{Cl}_{2}, 23{ }^{\circ} \mathrm{C}, \delta\right): 22.8\left({ }^{1} J_{\mathrm{P}-\mathrm{Pt}}=1292 \mathrm{~Hz}\right)$.

$\left(\kappa^{2}-\left({ }^{t} \mathrm{Bu}\right)_{2} \mathrm{PCMe}_{2} \mathrm{CH}_{2}\right) \operatorname{Pt}\left(\mathrm{Si}(\mathrm{OEt})_{3}\right)\left(\mathrm{SMe}_{2}\right)(57)$
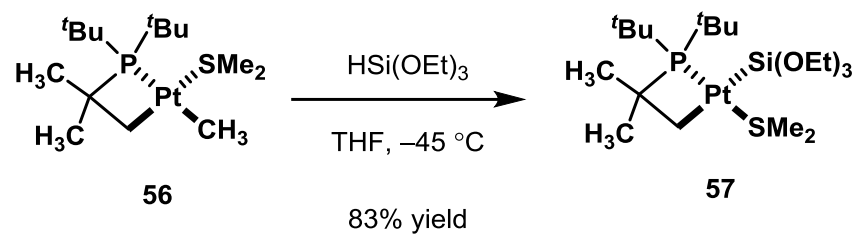

In a dry, $\mathrm{N}_{2}$-filled glovebox, platinum complex 57 (122 mg, $0.258 \mathrm{mmol}, 1.00$ equiv.), THF (6.0 mL), and a Teflon-coated magnetic stirring bar were added to a $20 \mathrm{~mL}$ glass scintillation vial. The vial was chilled at $-45^{\circ} \mathrm{C}$ for $15 \mathrm{~min}$. and triethoxysilane (48 $\mu \mathrm{L}, 42 \mathrm{mg}, 0.258 \mathrm{mmol}, 1.00$ equiv.) was added. The vial was warmed at $23{ }^{\circ} \mathrm{C}$ for 2 hours, then the clear colorless solution was concentrated under reduced pressure to yield the title compound as a white solid (133 $\mathrm{mg}, 83 \%)$. 
NMR Spectroscopy: ${ }^{1} \mathrm{H}$ NMR $\left(400 \mathrm{MHz}, \mathrm{C}_{6} \mathrm{D}_{6}, 23{ }^{\circ} \mathrm{C}, \delta\right): 4.15(\mathrm{q}, J=6.9 \mathrm{~Hz}, 6 \mathrm{H}), 2.42\left(\mathrm{~s},{ }^{2} J_{\mathrm{Pt}-\mathrm{H}}=29\right.$ $\mathrm{Hz}, 6 \mathrm{H}), 1.98\left(\mathrm{~d},{ }^{2} J_{\mathrm{Pt}-\mathrm{H}}=67 \mathrm{~Hz},{ }^{3} J_{\mathrm{P}-\mathrm{H}}=17 \mathrm{~Hz}, 2 \mathrm{H}\right), 1.61 .3(\mathrm{~m}, 33 \mathrm{H}) .{ }^{31} \mathrm{P}$ NMR $\left(162 \mathrm{MHz}, \mathrm{CD}_{2} \mathrm{Cl}_{2}, 23\right.$ $\left.{ }^{\circ} \mathrm{C}, \delta\right):-32.9\left({ }^{1} J_{\mathrm{P}-\mathrm{Pt}}=736 \mathrm{~Hz}\right)$.

\section{$\left[\left(\kappa^{2}-\left({ }^{t} \mathrm{Bu}\right)_{2} \mathrm{PCMe} \mathrm{CH}_{2}\right) \operatorname{Pt}\left(\mathrm{Si}(\mathrm{OEt})_{3}\right)\left(\mathrm{CH}_{2} \mathrm{CH}_{2} \mathrm{CH}=\mathrm{CH}_{2}\right)\right]\left[\mathrm{MgCl}\left(\mathrm{SMe}_{2}\right)\right](34)$}

Compound 34 was synthesized and used for hydrosilylation in situ without isolation. Attempts to isolate 34 resulted in isomerization and decomposition.

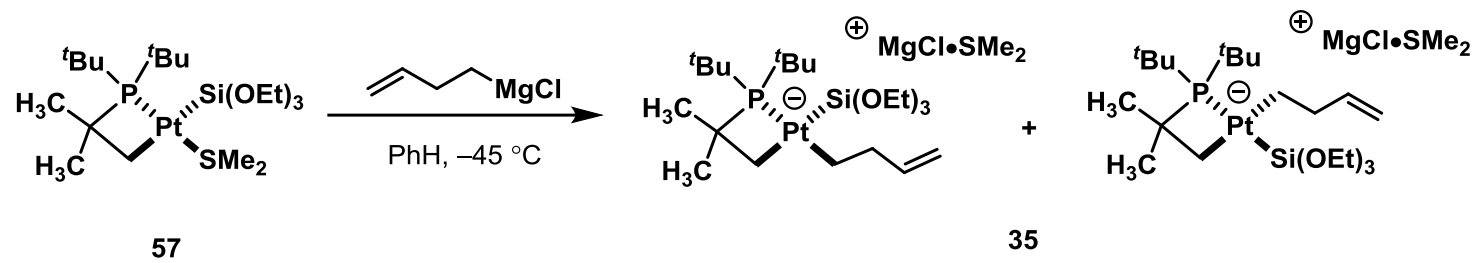

In a dry, $\mathrm{N}_{2}$-filled glovebox, platinum complex 57 (66 mg, $0.11 \mathrm{mmol}, 1.00$ equiv.), benzene (5 mL), and a Teflon-coated magnetic stirring bar were added to a $20 \mathrm{~mL}$ glass scintillation vial. The vial was chilled at $-45{ }^{\circ} \mathrm{C}$ for $30 \mathrm{~min}$., then butenylmagnesium chloride in THF $(\mathrm{c}=1.15 \mathrm{M}, 92 \mu \mathrm{L}, 0.11 \mathrm{mmol}, 1.00$ equiv.) was added and the vial was warmed at $23{ }^{\circ} \mathrm{C}$ for $30 \mathrm{~min}$. The product was analyzed by ${ }^{31} \mathrm{P}$ NMR, determined to contain a mixture of two isomers, and used in hydrosilylation without isolation. NMR Spectroscopy: ${ }^{31} \mathrm{P}$ NMR $\left(162 \mathrm{MHz}, \mathrm{C}_{6} \mathrm{D}_{6}, 23{ }^{\circ} \mathrm{C}, \delta\right): 23.3\left(\mathrm{~s},{ }^{1} \mathrm{~J}_{\mathrm{P}-\mathrm{Pt}}=1102 \mathrm{~Hz}\right), 9.5$ (s). 


\subsection{Synthesis of Butenylsilanes by Hydrosilylation}

\section{3-Butenyltriethoxysilane (18)}

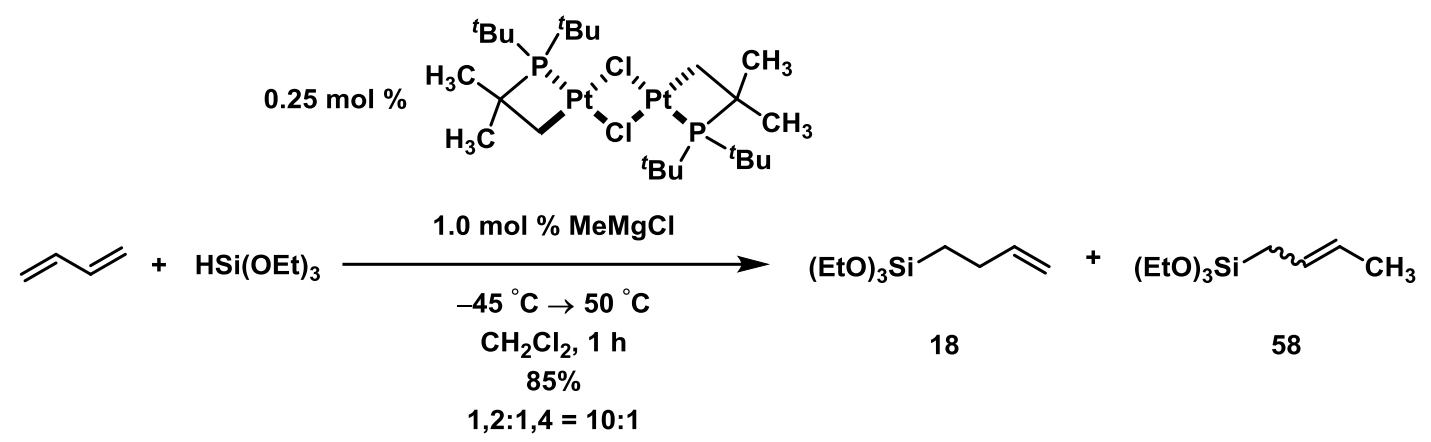

In a dry, $\mathrm{N}_{2}$-filled glovebox, platinum precatalyst $16(119.5 \mathrm{mg}, 0.1380 \mathrm{mmol}, 0.2500 \mathrm{~mol} \%)$, a Tefloncoated magnetic stirring bar, and dichloromethane $(15 \mathrm{~mL})$ were added to a $20 \mathrm{~mL}$ scintillation vial. After chilling at $-45{ }^{\circ} \mathrm{C}$ in a $\mathrm{CO}_{2} /{ }^{i} \mathrm{PrOH}$-cooled cold well for $30 \mathrm{~min}$., methylmagnesium chloride in THF was added ( $\mathrm{c}=3.28 \mathrm{M}, 168 \mu \mathrm{L}, 0.552 \mathrm{mmol}, 1.00 \mathrm{~mol} \%$ ) and the vial was sealed with a polyethylene-lined cap and shaken for 90 seconds at $23{ }^{\circ} \mathrm{C}$ to dissolve frozen droplets of Grignard reagent. The reaction mixture was stirred at $-45^{\circ} \mathrm{C}$ for 3 hours then at $23{ }^{\circ} \mathrm{C}$ for $30 \mathrm{~min}$., at which time precipitation of a white solid was observed. The vial was chilled at $-45{ }^{\circ} \mathrm{C}$ for $30 \mathrm{~min}$ then its contents were transferred to a precooled, $100 \mathrm{~mL}$ Schlenk vessel. A Teflon-coated magnetic stirring bar, butadiene (7.00 mL, $4.48 \mathrm{~g}, 83.0$ mmol, 1.50 equiv.), triethoxysilane (10.2 mL, $9.07 \mathrm{~g}, 55.2 \mathrm{mmol}, 1.00$ equiv.), and dichloromethane $(7.0 \mathrm{~mL})$ were added and the Schlenk vessel was sealed and removed from the glovebox. The reaction mixture was stirred at $50{ }^{\circ} \mathrm{C}$ in a pre-heated oil bath for one hour, then cooled at $23{ }^{\circ} \mathrm{C}$ for $5 \mathrm{~min}$. The tube was opened under ambient atmosphere and the contents decanted into a $50 \mathrm{~mL}$ round-bottom flask. Solvent was removed by rotary evaporation and the product was purified by bulb-to-bulb distillation ( 5 Torr, $\left.85^{\circ} \mathrm{C}\right)$ to yield butenyltriethoxysilane as a mixture of 1,2- and 1,4-addition products $(10.2 \mathrm{~g}, 10: 1$ 1,2-:1,4-addition, $85 \%$ ). Ratio of 1,2-:1,4-addition products determined to be $10: 1$ by ${ }^{1} \mathrm{H}$ NMR integration of signals corresponding to alkenyl protons (5.96-5.86 ppm for 3-butenyltriethoxysilane signal 
corresponds to $1 \mathrm{H} ; 5.49-5.37$ for 2-butenyltriethoxysilanes, overlapping signals for cis-and trans-isomers correspond to $2 \mathrm{H}$ ) and 91:9 by GC analysis $\left(\mathrm{HP}-5,6\right.$ psi, Program 1): $t_{\mathrm{R}}$ (major, 18) $=3.44$ min. $(90.6), t_{\mathrm{R}}$ $($ minor, 58) $=3.59 \min .(9.3)$.

NMR Spectroscopy: ${ }^{1} \mathrm{H}$ NMR $\left(600 \mathrm{MHz}, \mathrm{CD}_{2} \mathrm{Cl}_{2}, 25{ }^{\circ} \mathrm{C}, \delta\right): 5.92(\mathrm{~m}, 1 \mathrm{H}), 5.40-5.20(\mathrm{~m}) *, 5.0\left(\mathrm{dd},{ }^{3} J_{\mathrm{H}-\mathrm{H}}\right.$ $\left.=17.3 \mathrm{~Hz},{ }^{2} J_{\mathrm{H}-\mathrm{H}}=2.1 \mathrm{~Hz}, 1 \mathrm{H}\right), 4.90\left(\mathrm{dd},{ }^{3} J_{\mathrm{H}-\mathrm{H}}=10.0 \mathrm{~Hz},{ }^{2} J_{\mathrm{H}-\mathrm{H}}=1.8 \mathrm{~Hz}, 1 \mathrm{H}\right), 3.8\left(\mathrm{q},{ }^{3} J_{\mathrm{H}-\mathrm{H}}=7.0 \mathrm{~Hz}, 6 \mathrm{H}\right)$, $2.14(\mathrm{~m}, 2 \mathrm{H}), 1.2\left(\mathrm{t},{ }^{3} J_{\mathrm{H}-\mathrm{H}}=7.0 \mathrm{~Hz}, 9 \mathrm{H}\right), 1.63-1.72(\mathrm{~m})^{*}, 1.60-1.54(\mathrm{~m}) *, 0.7(\mathrm{~m}, 2 \mathrm{H}) .{ }^{13} \mathrm{C}$ NMR $(125$ $\left.\mathrm{MHz}, \mathrm{CD}_{2} \mathrm{Cl}_{2}, 25^{\circ} \mathrm{C}, \delta\right): 141.7,113.2,58.8,27.5,18.7,10.2$. HRMS-FIA (m/z): calcd for $\mathrm{C}_{10} \mathrm{H}_{22} \mathrm{O}_{3} \mathrm{SiNa}$ $[\mathrm{M}+\mathrm{Na}]^{+}, 241.1230$. Found, 241.1239. *Signals correspond to $c i s-$ and trans-triethoxysilylbut-2-ene (58).

\section{3-Methyl-3-butenyltriethoxysilane (20)}

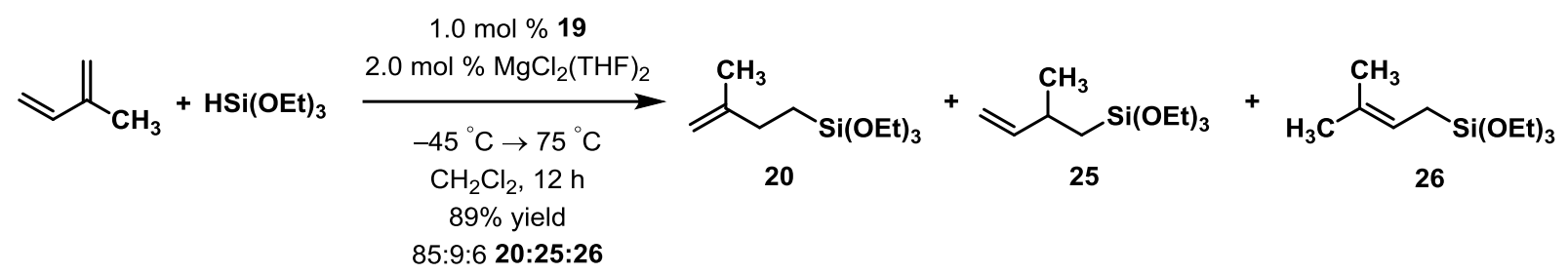

In a dry, $\mathrm{N}_{2}$ filled glovebox, platinum complex 19 (15.8 mg, $\left.24.0 \mu \mathrm{mol}, 1.00 \mathrm{~mol} \%\right), \mathrm{MgCl}_{2}(\mathrm{THF})_{2}(12.4$ $\mathrm{mg}, 51.8 \mu \mathrm{mol}, 2.16 \mathrm{~mol} \%)$, a Teflon-coated magnetic stirring bar, dichloromethane $(2 \mathrm{~mL})$, and tetrahydrofuran $(10 \mu \mathrm{L})$ were added to a $20 \mathrm{~mL}$ scintillation vial at $23{ }^{\circ} \mathrm{C}$ to form a white suspension. The reaction vial was chilled at $-45{ }^{\circ} \mathrm{C}$ in a $\mathrm{CO}_{2} / \mathrm{PrOH}$-cooled cold well for $30 \mathrm{~min}$. Isoprene $(240 \mu \mathrm{L}, 163$ mg, $2.40 \mathrm{mmol}, 1.00$ equiv.) and triethoxysilane (443 $\mu \mathrm{L}, 394 \mathrm{mg}, 2.40 \mathrm{mmol}, 1.00$ equiv.) were added and the reaction vial was capped with a polyethylene-lined cap and removed from the glovebox. The reaction mixture was stirred at $75{ }^{\circ} \mathrm{C}$ in a pre-heated aluminum heating block for $12 \mathrm{~h}$. The vial was opened under ambient atmosphere and the solvent was removed by rotary evaporation. The product was purified by bulb-to-bulb distillation $\left(145^{\circ} \mathrm{C}, 5\right.$ Torr) to afford the title compound as a colorless liquid (493 mg, $2.13 \mathrm{mmol}, 89 \%$ yield). Ratio of $\mathbf{2 0 : 2 5 : 2 6}$ determined to be 15:2:1 by GC analysis (HP-5, 6 psi, Program 2): $t_{\mathrm{R}}\left(\right.$ minor, 25) $=12.15(8.7), t_{\mathrm{R}}\left(\right.$ minor, 26) $=14.1(5.8), t_{\mathrm{R}}($ major, 20 $)=14.4(85.5)$. 
NMR Spectroscopy: ${ }^{1} \mathrm{H}$ NMR $\left(600 \mathrm{MHz}, \mathrm{CDCl}_{3}, 25{ }^{\circ} \mathrm{C}, \delta\right): 5.90-5.80(\mathrm{~m}) *, 5.21-5.12\left(\mathrm{t},{ }^{3} J_{\mathrm{H}-\mathrm{H}}=7.6\right.$ $\mathrm{Hz})^{* *}, 4.97\left(\mathrm{~d}, J_{\mathrm{H}-\mathrm{H}}=17.0 \mathrm{~Hz}\right)^{*}, 4.85\left(\mathrm{~d}, J_{\mathrm{H}-\mathrm{H}}=8.8 \mathrm{~Hz}\right)^{*}, 4.70\left(\mathrm{~d}, J_{\mathrm{H}-\mathrm{H}}=18.8 \mathrm{~Hz}, 1 \mathrm{H}\right), 3.83\left(\mathrm{q},{ }^{3} J_{\mathrm{H}-\mathrm{H}}=\right.$ $7.0 \mathrm{~Hz}, 6 \mathrm{H}), 2.42\left(\mathrm{sept},{ }^{3} J_{\mathrm{H}-\mathrm{H}}=6.5 \mathrm{~Hz}\right)^{*}, 2.17-2.03(\mathrm{~m}, 2 \mathrm{H}), 1.74(\mathrm{~s}, 3 \mathrm{H}), 1.70(\mathrm{~s})^{* *}, 1.62(\mathrm{~s})^{* *}, 1.55(\mathrm{~d}$, $\left.\left.{ }^{3} J_{\mathrm{H}-\mathrm{H}}=8.2 \mathrm{~Hz}\right)^{* *}, 1.24\left(\mathrm{t},{ }^{3} J_{\mathrm{H}-\mathrm{H}}=8.8 \mathrm{~Hz}\right), 9 \mathrm{H}\right), 1.09\left(\mathrm{~d},{ }^{3} J_{\mathrm{H}-\mathrm{H}}=7.0 \mathrm{~Hz}\right)^{*}, 0.83-0.74(\mathrm{~m}, 2 \mathrm{H}), 0.69\left(\mathrm{~d},{ }^{3} J_{H-}\right.$ $\mathrm{H}=8.2 \mathrm{~Hz})^{*}, 0.65\left(\mathrm{~d},{ }^{3} J_{\mathrm{H}-\mathrm{H}}=7.6 \mathrm{~Hz}\right) * .{ }^{13} \mathrm{C} \mathrm{NMR}\left(125 \mathrm{MHz}, \mathrm{CDCl}_{3}, 25{ }^{\circ} \mathrm{C}, \delta\right): 148.1,108.6,58.5,30.7$, 22.4, 18.5, 8.8. HRMS-FIA(m/z) calcd for $\mathrm{C}_{11} \mathrm{H}_{24} \mathrm{O}_{3} \mathrm{SiNa}[\mathrm{M}+\mathrm{Na}]^{+}, 255.1387$; found, 255.1381. *Signals correspond to minor isomer triethoxy(2-methylbut-3-en-1-yl)silane (25). **Signals correspond to minor isomer triethoxy(3-methylbut-2-en-1-yl)silane (26).

\section{Catalysis using precatalyst 16}

In a dry, $\mathrm{N}_{2}$-filled glovebox, platinum precatalyst $16(10.4 \mathrm{mg}, 12.0 \mu \mathrm{mol}, 0.50 \mathrm{~mol} \%)$, a Teflon-coated magnetic stirring bar, and dichloromethane $(1.0 \mathrm{~mL})$ were added to a $4 \mathrm{~mL}$ scintillation vial at $-45^{\circ} \mathrm{C}$ in a $\mathrm{CO}_{2} /{ }^{i} \mathrm{PrOH}$-cooled cold well to form a clear, colorless solution. After chilling at $-45{ }^{\circ} \mathrm{C}$ for 30 min., methylmagnesium chloride in THF ( $=3.28 \mathrm{M}, 14.6 \mu \mathrm{L}, 48.0 \mu \mathrm{mol}, 2.00 \mathrm{~mol} \%)$ was added and the reaction vessel was sealed with a Teflon-lined cap and shaken for 90 seconds at $23{ }^{\circ} \mathrm{C}$ to dissolve frozen droplets of Grignard reagent. The vial was cooled at $-45^{\circ} \mathrm{C}$ for 2.5 hours, warmed at $23{ }^{\circ} \mathrm{C}$ for 30 min., and cooled at $-45^{\circ} \mathrm{C}$ for $20 \mathrm{~min}$. Isoprene $(241 \mu \mathrm{L}, 166 \mathrm{mg}, 2.40 \mathrm{mmol}, 1.00$ equiv. $)$ and triethoxysilane (443 $\mu \mathrm{L}, 394 \mathrm{mg}, 2.40 \mathrm{mmol}, 1.00$ equiv.) were added. The reaction vessel was resealed, removed from the glovebox, and heated to $75{ }^{\circ} \mathrm{C}$ in a pre-heated aluminum heating block for 12 hours. The vial was opened under ambient atmosphere and the solvent was removed by rotary evaporation. The product was purified by bulb-to-bulb distillation $\left(150{ }^{\circ} \mathrm{C}, 5\right.$ Torr $)$ to afford the title compound $(\mathbf{2 0}+\mathbf{2 5}+\mathbf{2 6}$ only, 363 $\mathrm{mg}, 1.56 \mathrm{mmol}, 65 \%$ yield) as a mixture with residual triethoxysilane (12 w/w \%). Ratio of 20:25:26 determined to be $17: 1: 1$ by ${ }^{1} \mathrm{H}$ NMR integration of peaks in the alkenyl region $(5.18-5.14 \mathrm{ppm}$ for triethoxy(3-methylbut-2-en-1-yl)silane (26) signal corresponds to $1 \mathrm{H} ; 4.97-4.94$ for triethoxy(2methylbut-3-en-1-yl)silane (25) signal corresponds to $1 \mathrm{H} ; \quad 4.71-4.67$ for 3-methyl-3butenyltriethoxysilane (20) signal corresponds to $2 \mathrm{H}$ ). 


\section{Triethoxy(7-methyl-3-methyleneoct-6-en-1-yl)silane (21)}

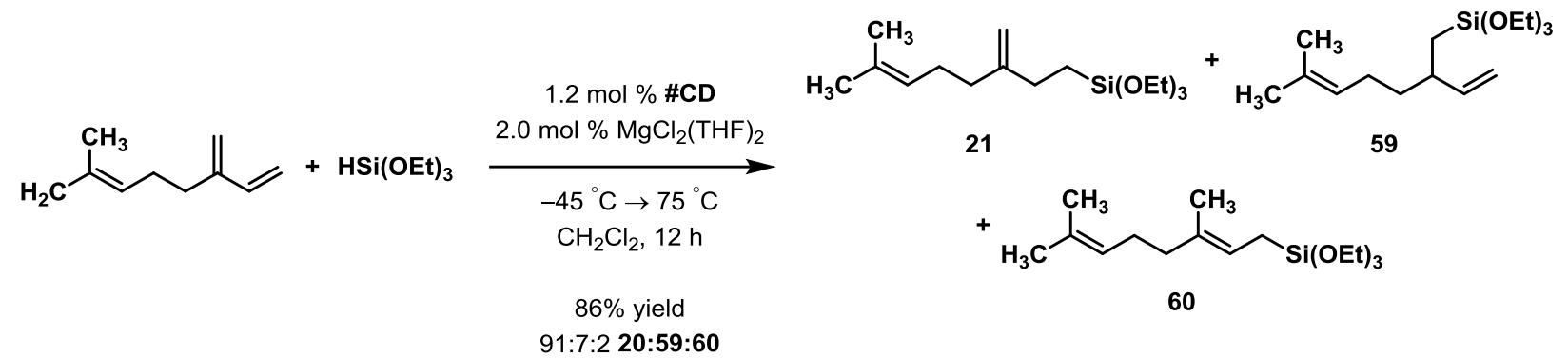

In a dry, $\mathrm{N}_{2}$ filled glovebox, platinum complex $19(16.1 \mathrm{mg}, 24.4 \mu \mathrm{mol}, 1.02 \mathrm{~mol} \%), \mathrm{MgCl}_{2}(\mathrm{THF})_{2}(12.2$ mg, $51.0 \mu \mathrm{mol}, 2.13 \mathrm{~mol} \%$ ), a Teflon-coated magnetic stirring bar, dichloromethane (2 $\mathrm{mL})$, and tetrahydrofuran $(10 \mu \mathrm{L})$ were added to a $20 \mathrm{~mL}$ scintillation vial at $23{ }^{\circ} \mathrm{C}$. The reaction vial was chilled at $-45{ }^{\circ} \mathrm{C}$ in a $\mathrm{CO}_{2} / \mathrm{PrOH}-$ cooled cold well for $30 \mathrm{~min}$. Myrcene $(414 \mu \mathrm{L}$ (total), $329 \mathrm{mg}, 2.10 \mathrm{mmol}$ (myrcene only), 1.00 equiv.) and triethoxysilane (443 $\mu \mathrm{L}, 2.40 \mathrm{mmol}, 1.00$ equiv.) were added. The reaction vial was sealed with a polyethylene-lined cap and removed from the glovebox. The reaction mixture was stirred at $75^{\circ} \mathrm{C}$ in a pre-heated aluminum heating block for 12 hours. The vial was opened under ambient atmosphere and the solvent was removed by rotary evaporation. Purification by bulb-tobulb distillation $\left(200^{\circ} \mathrm{C}, 5\right.$ Torr) afforded the title compound as a colorless liquid (543 $\mathrm{mg}, 1.81 \mathrm{mmol}$, $89 \%$ yield). The commercial myrcene used in this experiment contains $15 \%$ other terpene isomers with identical mass and similar boiling point. Yield and catalyst loading are based on myrcene content of starting material only. Ratio of 21:59:60 determined to be 53:4:1 by GC analysis (HP-5, 6 psi, Program $2): t_{\mathrm{R}}\left(\right.$ minor, 59) $=6.23(7.6), t_{\mathrm{R}}($ major, 21 $)=6.67(90.7), t_{\mathrm{R}}($ minor, 60 $)=8.78(1.7)$.

NMR Spectroscopy: ${ }^{1} \mathrm{H}$ NMR (600 MHz, $\left.\mathrm{CDCl}_{3}, 25^{\circ} \mathrm{C}, \delta\right): 5.70-5.62(\mathrm{~m}) *, 5.20(\mathrm{td}, J=8.0,1.0 \mathrm{~Hz})^{* *}$, 5.15-5.07 (m, 1H), 5.04-4.91 (m)*, $4.76(\mathrm{~s}, 1 \mathrm{H}), 4.70(\mathrm{~s}, 1 \mathrm{H}), 3.81\left(\mathrm{q},{ }^{3} J_{\mathrm{H}-\mathrm{H}}=7.0 \mathrm{~Hz}, 6 \mathrm{H}\right), 2.28-2.16$ $(\mathrm{m})^{*}, 2.14-2.02(\mathrm{~m}, 6 \mathrm{H}), 1.98-2.01(\mathrm{~m})^{* *}, 1.68(\mathrm{~s}, 3 \mathrm{H}), 1.60(\mathrm{~s}, 3 \mathrm{H}), 1.55(\mathrm{~d}, J=8.0 \mathrm{~Hz})^{* *}, 1.23\left(\mathrm{t},{ }^{3} J_{\mathrm{H}-}\right.$ н $=7.0 \mathrm{~Hz}), 0.82-0.77(\mathrm{~m}, 2 \mathrm{H}), 0.76-0.72(\mathrm{~m}) * .{ }^{13} \mathrm{C} \mathrm{NMR}\left(125 \mathrm{MHz}, \mathrm{CDCl}_{3}, 25{ }^{\circ} \mathrm{C}, \delta\right): 151.8,131.6$, $124.4,107.7,58.5,36.0,29.1,26.7,25.8,18.4,17.8,8.6$. HRMS-FIA(m/z) calcd for $\mathrm{C}_{16} \mathrm{H}_{32} \mathrm{O}_{3} \mathrm{SiNa}$ 
$[\mathrm{M}+\mathrm{Na}]^{+}$, 323.2022; found, 323.2013. *Signals correspond to minor isomer triethoxy(6-methyl-2vinylhept-5-en-1-yl)silane (59). **Signals correspond to minor isomer (E)-(3,7-dimethylocta-2,6-dien-1yl)triethoxysilane (60).

\section{(2,3-Dimethylbut-3-en-1-yl)triethoxysilane (22)}

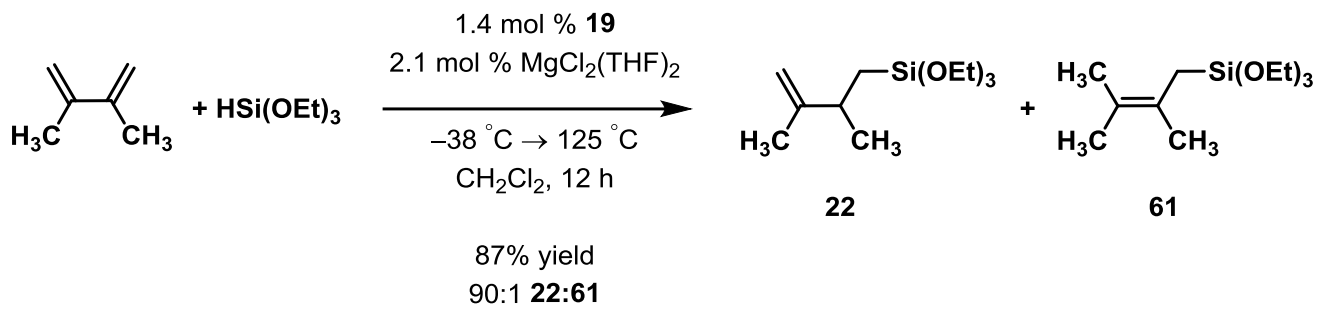

In a dry, $\mathrm{N}_{2}$ filled glovebox, platinum complex $19(21.4 \mathrm{mg}, 32.4 \mu \mathrm{mol}, 1.35 \mathrm{~mol} \%), \mathrm{MgCl}_{2}(\mathrm{THF})_{2}(12.1$ mg, $50.5 \mu \mathrm{mol}, 2.10 \mathrm{~mol} \%)$, a Teflon-coated magnetic stirring bar, dichloroethane (2 mL), and tetrahydrofuran $(10 \mu \mathrm{L})$ were added to a $20 \mathrm{~mL}$ scintillation vial at $23{ }^{\circ} \mathrm{C}$. The reaction vial was chilled at $-38{ }^{\circ} \mathrm{C}$ in a $\mathrm{CO}_{2} / \mathrm{iPrOH}-$ cooled cold well for $30 \mathrm{~min}$. 2,3-Dimethylbutadiene $(274 \mu \mathrm{L}, 198 \mathrm{mg}, 2.40$ mmol, 1.00 equiv.) and triethoxysilane (443 $\mu \mathrm{L}, 394 \mathrm{mg}, 2.40 \mathrm{mmol}, 1.00$ equiv.) were added. The reaction vial was sealed with a polyethylene-lined cap and removed from the glovebox. The reaction mixture was stirred at $125^{\circ} \mathrm{C}$ in a pre-heated aluminum heating block for 12 hours. The vial was opened under ambient atmosphere and the solvent was removed by rotary evaporation. Purification by bulb-tobulb distillation $\left(110^{\circ} \mathrm{C}, 5\right.$ Torr) afforded the title compound as a colorless liquid $(512 \mathrm{mg}, 2.08 \mathrm{mmol}$, 87\%). Ratio of 1,2-:1,4-addition determined to be $90: 1$ by GC analysis (HP-5, 6 psi, Program 2): $t_{\mathrm{R}}$ $\left(\right.$ major, 22) $=4.29(98.9), t_{\mathrm{R}}($ minor, 61) $=4.48(1.1)$.

NMR Spectroscopy: ${ }^{1} \mathrm{H}$ NMR $\left(600 \mathrm{MHz}, \mathrm{CDCl}_{3}, 25{ }^{\circ} \mathrm{C}, \delta\right): 4.72(\mathrm{~s}, 1 \mathrm{H}), 4.61(\mathrm{~s}, 1 \mathrm{H}), 3.82\left(\mathrm{q},{ }^{3} J_{\mathrm{H}-\mathrm{H}}=7.0\right.$ $\mathrm{Hz}, 6 \mathrm{H}), 2.46-2.39(\mathrm{~m}, 2 \mathrm{H}), 1.70(\mathrm{~s}, 3 \mathrm{H}), 1.23\left(\mathrm{t},{ }^{3} \mathrm{~J}_{\mathrm{H}-\mathrm{H}}=7.0 \mathrm{~Hz}, 9 \mathrm{H}\right), 0.88-0.64(\mathrm{~m}, 2 \mathrm{H}) .{ }^{13} \mathrm{C}$ NMR $(125$ $\left.\mathrm{MHz}, \mathrm{CDCl}_{3}, 25{ }^{\circ} \mathrm{C}, \delta\right): 152.6,108.0,58.4,36.1,22.0,19.2,18.4,17.5$. HRMS-FIA(m/z) calcd for $\mathrm{C}_{12} \mathrm{H}_{26} \mathrm{O}_{3} \mathrm{SiNa}[\mathrm{M}+\mathrm{Na}]^{+}, 269.1543$; found, 269.1537. 


\section{Benzyl 4-methylene-6-(triethoxysilyl)hexanoate (23)}

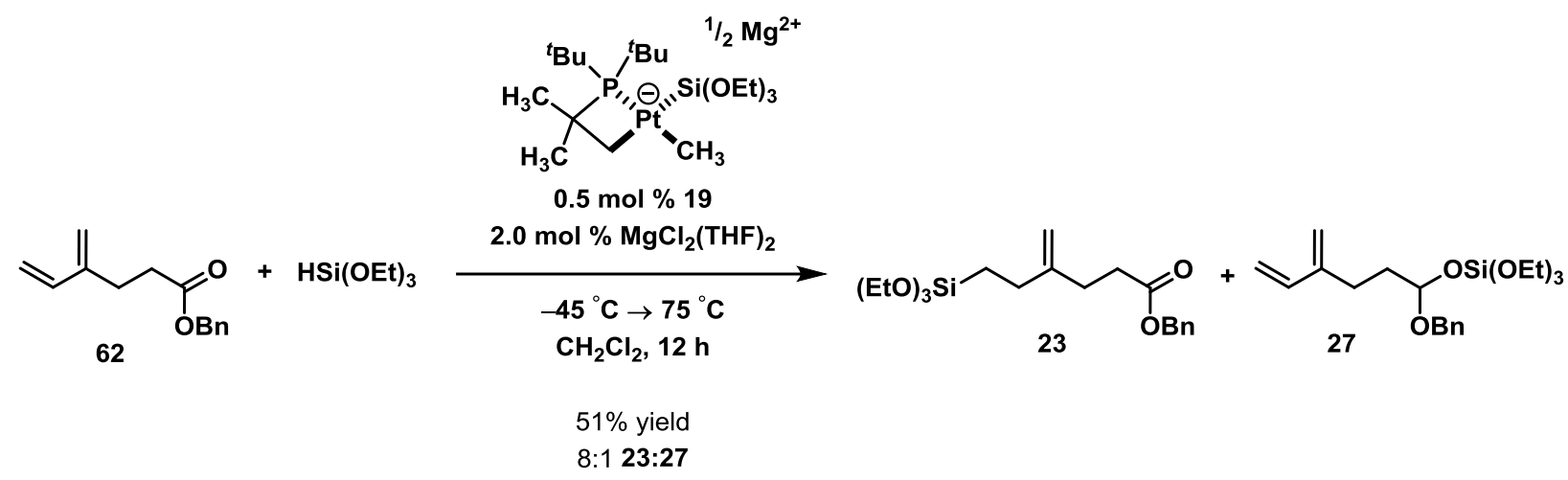

In a dry, $\mathrm{N}_{2}$ filled glovebox, platinum complex 19 (15.8 mg, $\left.12.0 \mu \mathrm{mol}, 0.500 \mathrm{~mol} \%\right), \mathrm{MgCl}_{2}(\mathrm{THF})_{2}$ (11.5 mg, $48.0 \mu \mathrm{mol}, 2.00 \mathrm{~mol} \%)$, dichloromethane $(2 \mathrm{~mL})$, THF $(10 \mu \mathrm{L})$, and a Teflon-coated stirring bar were added to a $20 \mathrm{~mL}$ glass scintillation vial. The vial was chilled at $-45^{\circ} \mathrm{C}$ in a $\mathrm{CO}_{2} /{ }^{i} \mathrm{PrOH}$-cooled cold well for $30 \mathrm{~min}$, then the mixture was transferred to a $20 \mathrm{~mL}$ scintillation vial containing benzyl 4methylenehex-5-enoate (519 mg, $2.40 \mathrm{mmol}, 1.00$ equiv.) and triethoxysilane (443 $\mu \mathrm{L}, 2.40 \mathrm{mmol}, 220$. mg, 1.00 equiv.) was added before the vial was sealed with a polyethylene-lined cap. The sealed reaction vial was removed from the glovebox and the mixture was stirred at $75{ }^{\circ} \mathrm{C}$ in a pre-heated aluminum heating block for $12 \mathrm{~h}$. The vial was opened under ambient atmosphere and the solvent was removed under reduced pressure. Purification by Kugelrohr distillation $\left(250{ }^{\circ} \mathrm{C}, 4\right.$ Torr $)$ afforded the title compound as a colorless liquid (464 mg, 51\%).

NMR Spectroscopy: ${ }^{1} \mathrm{H}$ NMR (600 MHz, $\left.\mathrm{CDCl}_{3}, 23{ }^{\circ} \mathrm{C}, \delta\right): 7.38-7.31(\mathrm{~m}, 5 \mathrm{H}), 5.12(\mathrm{~s}, 2 \mathrm{H}), 4.74(\mathrm{~d}$, $J=58.4 \mathrm{~Hz}, 1 \mathrm{H}), 3.82(\mathrm{q}, J=6.98 \mathrm{~Hz}, 6 \mathrm{H}), 2.53-2.33(\mathrm{~m}, 4 \mathrm{H}), 2.13-2.10(\mathrm{~m}, 2 \mathrm{H}), 1.23$ (t, $J=7.01 \mathrm{~Hz}$, $9 \mathrm{H}), 0.78-0.75(\mathrm{~m}, 2 \mathrm{H})$. 


\section{(1R,4R)-bicyclo[2.2.1]hept-5-en-2S-yltriethoxysilane (24)}

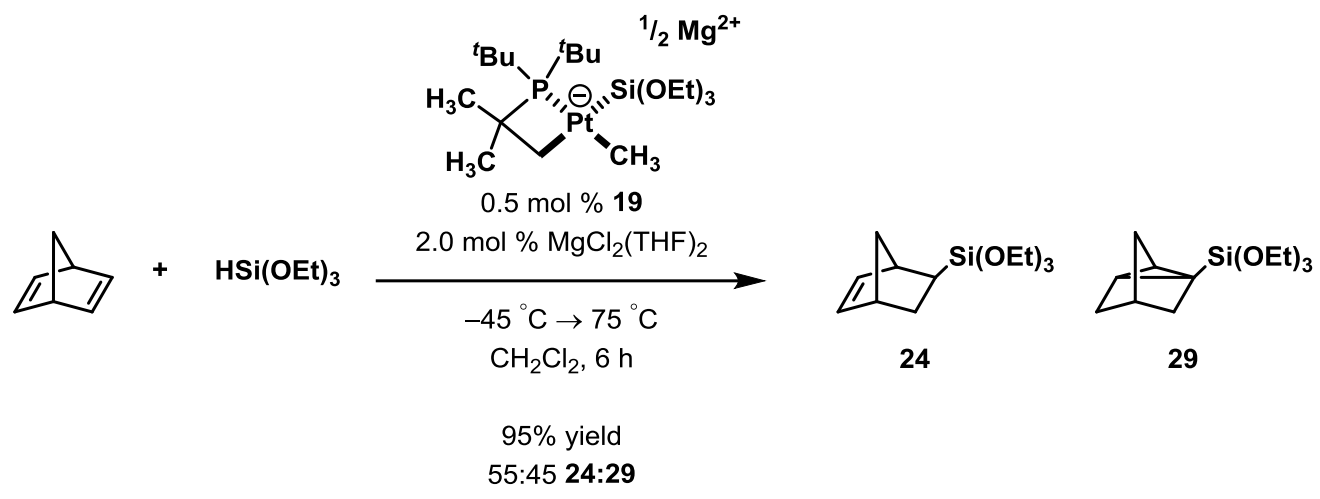

In a dry, $\mathrm{N}_{2}$ filled glovebox, platinum complex $19(16.7 \mathrm{mg}, 12.7 \mu \mathrm{mol}, 0.529 \mathrm{~mol} \%), \mathrm{MgCl}_{2}(\mathrm{THF})_{2}$ (12.1 mg, $50.5 \mu \mathrm{mol}, 2.10 \mathrm{~mol} \%$ ), a Teflon-coated stirring bar, dichloromethane $(2 \mathrm{~mL})$, and THF (10 $\mu \mathrm{L}$ ) were added to a $20 \mathrm{~mL}$ glass scintillation vial to form a pale yellow suspension. The vial was chilled at $-45{ }^{\circ} \mathrm{C}$ in a $\mathrm{CO}_{2} /{ }^{i} \mathrm{PrOH}$-cooled cold well for $10 \mathrm{~min}$., then norbornadiene (243 $\mu \mathrm{L}, 2.40 \mathrm{mmol}, 220$. mg, 1.00 equiv.) and triethoxysilane (443 $\mu \mathrm{L}, 2.40 \mathrm{mmol}, 394 \mathrm{mg}, 1.00$ equiv.) were added. The vial was sealed with a polyethylene-lined cap and removed from the glovebox, then the mixture was stirred at $75^{\circ}$ $\mathrm{C}$ in a pre-heated aluminum heating block for $6 \mathrm{~h}$. The vial was opened under ambient atmosphere and the solvent was removed under reduced pressure. Purification by Kugelrohr distillation $\left(140{ }^{\circ} \mathrm{C}, 5\right.$ Torr $)$ afforded the title compound as a colorless liquid (581 mg, $2.27 \mathrm{mmol}, 95 \%)$.

NMR Spectroscopy: ${ }^{1} \mathrm{H}$ NMR $\left(600 \mathrm{MHz}, \mathrm{CDCl}_{3}, 23{ }^{\circ} \mathrm{C}, \delta\right): 6.03-5.99(\mathrm{~m}, 4 \mathrm{H}) 3.84(\mathrm{q}, J=7.00 \mathrm{~Hz}, 6 \mathrm{H})$, $3.78(\mathrm{q}, J=7.02 \mathrm{~Hz}, 6 \mathrm{H}), 3.03-3.01(\mathrm{~m}, 1 \mathrm{H}), 2.89-2.86(\mathrm{~m}, 1 \mathrm{H}), 1.87(\mathrm{ddd}, J=11.0,9.71,3.74 \mathrm{~Hz}$, 1H), 1.56-1.54 (m, 1H), 1.27-1.25 (m, 1H), $1.23(\mathrm{t}, J=6.98 \mathrm{~Hz}, 9 \mathrm{H}), 1.20(\mathrm{t}, J=7.00 \mathrm{~Hz}, 9 \mathrm{H}), 1.16-$ $1.01(\mathrm{~m}, 6 \mathrm{H}), 0.81-0.79(\mathrm{~m}, 1 \mathrm{H}) .{ }^{13} \mathrm{C} \mathrm{NMR}\left(125 \mathrm{MHz}, \mathrm{CDCl}_{3}, 25{ }^{\circ} \mathrm{C}, \delta\right): 135.4,134.8,58.5,58.4,51.0$ $44.3,42.3,35.9,31.7,31.6,28.8,27.1,20.9,18.4,12.1,10.4,10.4$. HRMS-FIA(m/z) calcd for 
$\mathrm{C}_{13} \mathrm{H}_{24} \mathrm{O}_{3} \mathrm{SiNa}[\mathrm{M}+\mathrm{Na}]+, 279.1387$; found, 279.1382. Spectroscopic data shows the title compound as a 55:45 mixture of 28:29, which correspond to previously reported spectral data. ${ }^{14}$

\section{(E)-Triethoxy(pent-2-en-1-yl)silane (30)}

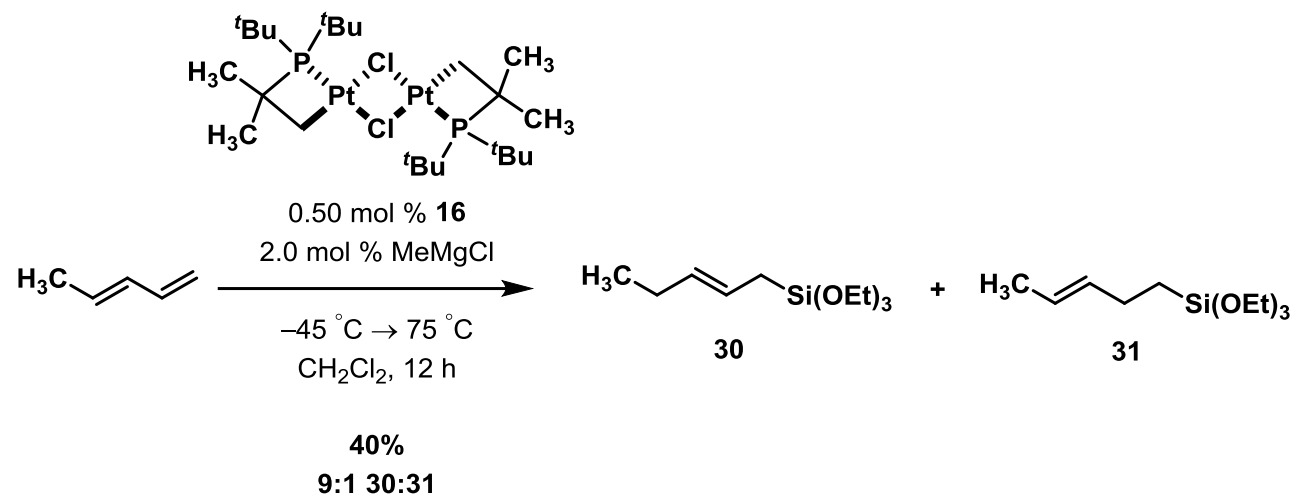

In a dry, $\mathrm{N}_{2}$-filled glovebox, platinum precatalyst $16(10.4 \mathrm{mg}, 12.0 \mu \mathrm{mol}, 0.50 \mathrm{~mol} \%)$, a Teflon-coated magnetic stirring bar, and dichloromethane $(1.0 \mathrm{~mL})$ were added to a $4 \mathrm{~mL}$ scintillation vial at $-45^{\circ} \mathrm{C}$ in a $\mathrm{CO}_{2} /{ }^{i} \mathrm{PrOH}$-cooled cold well to form a clear, colorless solution. After chilling at $-45{ }^{\circ} \mathrm{C}$ for 30 min., methylmagnesium chloride in THF ( $\mathrm{c}=3.28 \mathrm{M}, 14.6 \mu \mathrm{L}, 48.0 \mu \mathrm{mol}, 2.00 \mathrm{~mol} \%)$ was added and the reaction vessel was sealed with a Teflon-lined cap and shaken for 90 seconds at $23{ }^{\circ} \mathrm{C}$ to dissolve frozen droplets of Grignard reagent. The vial was cooled at $-45^{\circ} \mathrm{C}$ for 2.5 hours, warmed at $23{ }^{\circ} \mathrm{C}$ for 30 min., and cooled at $-45^{\circ} \mathrm{C}$ for $20 \mathrm{~min}$. 1,3-trans-Pentadiene (240 $\mu \mathrm{L}, 164 \mathrm{mg}, 2.40 \mathrm{mmol}, 1.00$ equiv.) and triethoxysilane (443 $\mu \mathrm{L}, 394 \mathrm{mg}, 2.40 \mathrm{mmol}, 1.00$ equiv.) were added. The reaction vessel was resealed, removed from the glovebox, and heated to $50{ }^{\circ} \mathrm{C}$ in a pre-heated aluminum heating block for 12 hours. The vial was opened under ambient atmosphere and the solvent was removed by rotary evaporation $(0.215 \mathrm{~g}, 39 \%)$. The product was analyzed by ${ }^{1} \mathrm{H}$ NMR; ratio of $\mathbf{3 0 : 3 1}$ determined to be $9: 1$ by ${ }^{1} \mathrm{H}$ NMR integration of peaks in the alkyl region. These data correspond to reported spectral data.

\footnotetext{
${ }^{14}$ Kuivila, H. G.; Warner, C. R. J. Org. Chem. 1964, 29, 2845.
} 
NMR Spectroscopy: ${ }^{1} \mathrm{H}$ NMR (400 MHz, $\left.\mathrm{CDCl}_{3}, 23{ }^{\circ} \mathrm{C}, \delta\right): 5.5-5.3(\mathrm{~m}, 2 \mathrm{H}), 3.95-3.75$ (q, $J=7.8 \mathrm{~Hz}$, 6H), 2.06 (quint., $J=7.8 \mathrm{~Hz}, 2 \mathrm{H}), 1.62$ (t, $J=7.8 \mathrm{~Hz}, 2 \mathrm{H}), 1.23(\mathrm{t}, J=6.3 \mathrm{~Hz}, 9 \mathrm{H}), 0.97$ (t, $J=7.0 \mathrm{~Hz}$, $3 \mathrm{H})$.

\subsection{Synthesis of Reagents, Ligands, and Substrates}

\section{2-Methyl-6-methyleneoct-7-ene-2,3-diol (63)}

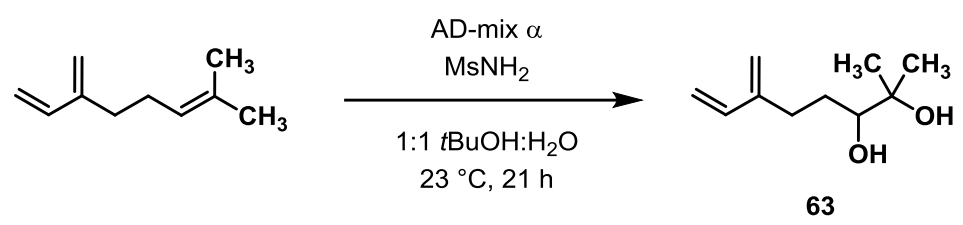

The synthesis of $\mathbf{6 3}$ was adapted from the method of Wu et al. ${ }^{15}$ To AD-mix $(50.3 \mathrm{~g}, 0.685 \mathrm{~g} / \mathrm{mmol}$ myrcene $)$ in $1.2: 1 \mathrm{v} / \mathrm{v}{ }^{t} \mathrm{BuOH} / \mathrm{H}_{2} \mathrm{O}(550 \mathrm{~mL})$ at $23{ }^{\circ} \mathrm{C}$ was added methanesulfonamide $(7.19 \mathrm{~g}, 76.0 \mathrm{mmol}$, 1.03 equiv.). The reaction mixture was stirred vigorously until the mixture became homogeneous, then myrcene (10.0 g, $12.59 \mathrm{~mL}, 73.4 \mathrm{mmol}, 1.00$ equiv.) was added and the reaction mixture was stirred at 23 ${ }^{\circ} \mathrm{C}$ for $21.5 \mathrm{~h}$. To the reaction mixture was added $\mathrm{Na}_{2} \mathrm{~S}_{2} \mathrm{O}_{3} \cdot 5 \mathrm{H}_{2} \mathrm{O}(155 \mathrm{~g}, 624$ mmol, 8.50 equiv. $)$ and the reaction mixture was stirred for $6 \mathrm{~h}$ at $23{ }^{\circ} \mathrm{C}$. Water $(100 \mathrm{~mL})$ was added and the aqueous layer was extracted with EtOAc $(3 \times 100 \mathrm{~mL})$. The combined organic layers were washed with brine $(100 \mathrm{~mL})$, dried over $\mathrm{Na}_{2} \mathrm{SO}_{4}$, filtered, and concentrated under reduced pressure. The residue was used for the synthesis of $\mathbf{6 3}$ without further purification.

\footnotetext{
${ }^{15}$ Wu, J. Y.; Stanzl, B. N.; Ritter, T. J. Am. Chem. Soc. 2010, 132, 13214.
} 


\section{4-Methylenehex-5-enal (64)}

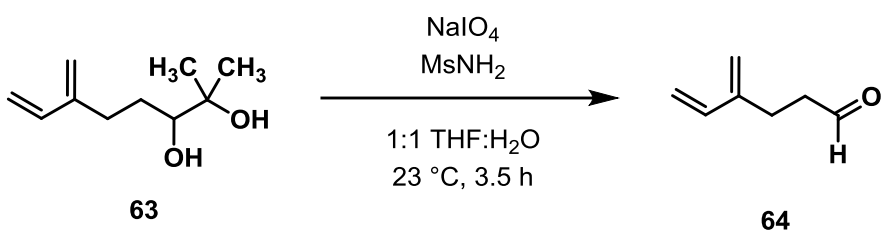

The synthesis of $\mathbf{6 4}$ was adapted from the method of Wu et al. ${ }^{15}$ To the residue of $\mathbf{6 3}$ carried through from dihydroxylation (11.3 g, $66.4 \mathrm{mmol}, 1.00$ equiv.) in $1: 1 \mathrm{v} / \mathrm{v}$ THF/ $\mathrm{H}_{2} \mathrm{O}(644 \mathrm{~mL})$ was added $\mathrm{NaIO}_{4}(42.6 \mathrm{~g}$, 199 mmol, 3.00 equiv.) and the reaction mixture was stirred for $3.5 \mathrm{~h}$ at $23{ }^{\circ} \mathrm{C}$. Water $(100 \mathrm{~mL})$ was added and the aqueous layer was extracted with $\mathrm{Et}_{2} \mathrm{O}(3 \times 150 \mathrm{~mL})$. The combined organic layers were washed with brine $(1 \times 60 \mathrm{~mL})$, dried over $\mathrm{Na}_{2} \mathrm{SO}_{4}$, filtered, and concentrated carefully under reduced pressure. The residue was purified by chromatography on silica gel, eluting with $5 \% \mathrm{v} / \mathrm{v}$ EtOAc/hexanes to give the title compound as a colorless oil (1.70 g, $15.4 \mathrm{mmol}, 21 \%$ over two steps).

NMR Spectroscopy: ${ }^{1} \mathrm{H}$ NMR (400 MHz, CDCl3, $\left.25{ }^{\circ} \mathrm{C}\right): 9.79$ (t, $\left.J=1.53,1 \mathrm{H}\right), 6.37$ (dd, $J=17.7,10.8$ $\mathrm{Hz}, 1 \mathrm{H}), 5.22(\mathrm{~d}, J=17.6 \mathrm{~Hz}, 1 \mathrm{H}), 5.10(\mathrm{~d}, J=10.9 \mathrm{~Hz}, 1 \mathrm{H}), 5.06-5.05(\mathrm{~m}, 1 \mathrm{H}), 5.00-4.99(\mathrm{~m}, 1 \mathrm{H})$ 2.67-2.53 $(\mathrm{m}, 4 \mathrm{H})$. These spectroscopic data correspond to previously reported data.

\section{4-Methylenehex-5-enoic Acid (65)}

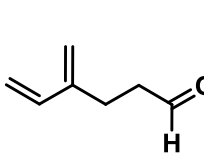

64

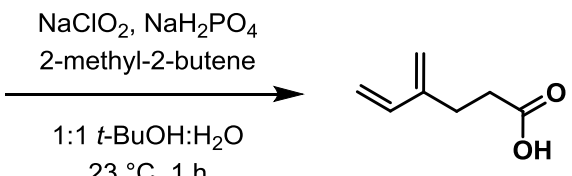

65

The synthesis of $\mathbf{6 5}$ was adapted from the method of $\mathrm{Wu}$ et al. ${ }^{15}$ In air, $\mathrm{NaH}_{2} \mathrm{PO}_{4} \cdot \mathrm{H}_{2} \mathrm{O}(2.26 \mathrm{~g}, 16.4$ mmol, 1.50 equiv.), 2-methyl-2-butene (2.32 g, $3.52 \mathrm{~mL}, 33.1 \mathrm{mmol}, 3.04$ equiv.), and $\mathrm{NaClO}_{2}(0.990 \mathrm{~g}$, 10.9 mmol, 1.00 equiv.) were added to a solution of 4-methylenehex-5-enal (64) (1.20 g, 10.9 mmol, 1.00 equiv.) in $1: 1 \mathrm{v} / \mathrm{v}{ }^{t} \mathrm{BuOH} / \mathrm{H}_{2} \mathrm{O}(22 \mathrm{~mL})$. The reaction was stirred at $23{ }^{\circ} \mathrm{C}$ for $1.5 \mathrm{~h}$, diluted with sat. 
$\mathrm{Na}_{2} \mathrm{CO}_{3}$-solution $(15 \mathrm{~mL})$ and the aqueous layer was extracted with $\mathrm{Et}_{2} \mathrm{O}(2 \times 10 \mathrm{~mL})$ and $\mathrm{EtOAc}(1 \times 10$ $\mathrm{mL}$ ). Hydrochloric acid ( $3 \mathrm{M}$ in $\mathrm{H}_{2} \mathrm{O}, 15 \mathrm{~mL}$ ) was added to the aqueous layer and it was extracted with dichloromethane $(3 \times 10 \mathrm{~mL})$. The combined organic layers were dried over $\mathrm{Na}_{2} \mathrm{SO}_{4}$. Evaporation of the solvent afforded the title compound as a yellow oil (648 mg, $5.14 \mathrm{mmol}, 47 \%)$.

NMR Spectroscopy: ${ }^{1} \mathrm{H}$ NMR $\left(400 \mathrm{MHz}, \mathrm{CDCl} 3,25^{\circ} \mathrm{C}\right): 6.38(\mathrm{dd}, J=17.7,10.8 \mathrm{~Hz}, 1 \mathrm{H}), 5.25(\mathrm{~d}, J=$ $17.7 \mathrm{~Hz}, 1 \mathrm{H}), 5.10(\mathrm{~d}, J=10.9 \mathrm{~Hz}, 1 \mathrm{H}), 5.07(\mathrm{~s}, 1 \mathrm{H}), 5.03(\mathrm{~s}, 1 \mathrm{H}) 1 \mathrm{H}), 2.58-2.55(\mathrm{~m}, 4 \mathrm{H})$. These spectroscopic data correspond to previously reported data. ${ }^{15}$

\section{Benzyl 4-methylenehex-5-enoate (62)}

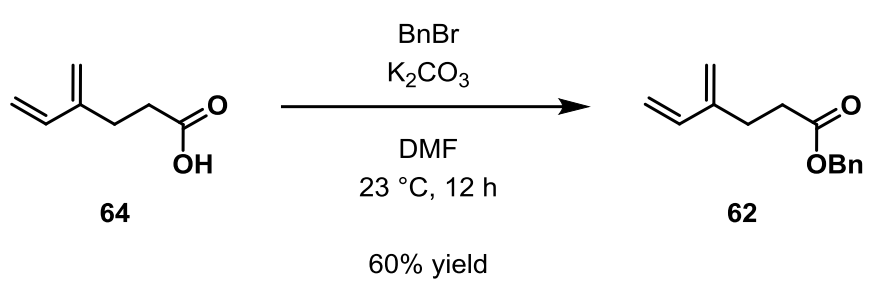

4-Methylenehex-5-enoic acid (64, $647.8 \mathrm{mg}, 5.14 \mathrm{mmol}, 1.00$ equiv.) was dissolved in DMF (5.0 mL). Benzyl bromide $(0.730 \mathrm{~mL}, 1.06 \mathrm{mmol}, 1.20$ equiv.) and potassium carbonate $(1.28 \mathrm{~g}, 9.23 \mathrm{mmol}, 1.50$ equiv.) were added and the reaction mixture was stirred at $23{ }^{\circ} \mathrm{C}$ for $12 \mathrm{~h}$. Ethyl acetate $(10 \mathrm{~mL})$ and $\mathrm{H}_{2} \mathrm{O}$ $(5 \mathrm{~mL})$ were added. The aqueous layer was extracted with ethyl acetate $(3 \times 20 \mathrm{~mL})$. The combined organic layers were washed with $\mathrm{H}_{2} \mathrm{O}(3 \times 10 \mathrm{~mL})$ and brine $(10 \mathrm{~mL})$, dried over $\mathrm{Na}_{2} \mathrm{SO}_{4}$ and the solvent was removed under reduced pressure. Purification by column chromatography on silica gel 5\% v/v EtOAc/hexanes afforded the title compound as colorless liquid (0.668 g, $3.09 \mathrm{mmol}, 60 \%)$.

TLC (5\% v/v EtOAc/hexanes): $R_{\mathrm{f}}=0.30$. NMR Spectroscopy: ${ }^{1} \mathrm{H}$ NMR $\left(400 \mathrm{MHz}, \mathrm{CDCl}_{3}, 25{ }^{\circ} \mathrm{C}\right): 7.37$ (m, 5H), $6.37(\mathrm{dd}, J=17.6,10.9 \mathrm{~Hz}, 1 \mathrm{H}), 5.25(\mathrm{~d}, J=17.7 \mathrm{~Hz}, 1 \mathrm{H}), 5.09$ (d, $J=10.9 \mathrm{~Hz}, 1 \mathrm{H}), 5.04(\mathrm{~s}$, 1H), $5.00(\mathrm{~s}, 1 \mathrm{H}), 2.60-2.57(\mathrm{~m}, 4 \mathrm{H}) .{ }^{13} \mathrm{C} \mathrm{NMR}\left(125 \mathrm{MHz}, \mathrm{CDCl}_{3}, 25{ }^{\circ} \mathrm{C}\right): 173.1,144.7,138.5,136.1$, $128.7,128.4,116.3,113.7,66.4,33.1,26.5$. 


\section{Di-tert-butyl-ortho-tolylphosphine (47)}

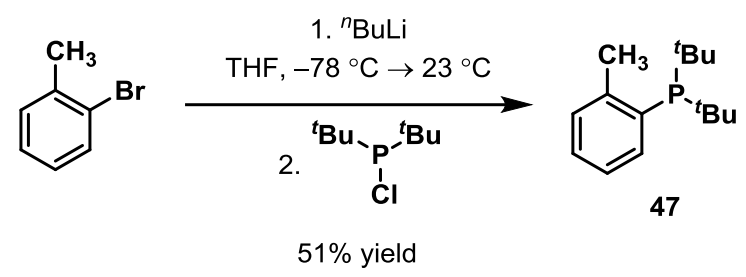

The preparation of $\mathbf{4 7}$ was adapted from the procedure used by Cheney and Shaw. ${ }^{12}$ Under an atmosphere of dry $\mathrm{N}_{2}$, 2-Bromotoluene (3.00 mL, $4.27 \mathrm{~g}, 24.9$ mmol, 1.20 equiv.), $\mathrm{Et}_{2} \mathrm{O}$ (100 mL), and a Tefloncoated magnetic stirring bar were added to an oven-dried three-necked $250 \mathrm{~mL}$ flask. The flask was cooled in a $\mathrm{CO}_{2} /$ acetone bath at $-78{ }^{\circ} \mathrm{C}$ and ${ }^{n} \mathrm{BuLi}$ in hexanes $(\mathrm{c}=2.5 \mathrm{M}, 10.4 \mathrm{~mL}, 26.0 \mathrm{mmol}, 1.25$ equiv.) was added dropwise over $35 \mathrm{~min}$. The clear yellow solution was stirred at $-78{ }^{\circ} \mathrm{C}$ for 1.5 hours, then warmed at $0{ }^{\circ} \mathrm{C}$ for $30 \mathrm{~min}$. in an ice bath, then warmed at $23{ }^{\circ} \mathrm{C}$ for $30 \mathrm{~min}$, at which point the solution was observed to be clear and colorless. The solution was cooled to $0{ }^{\circ} \mathrm{C}$, then di-tertbutylchlorophosphine (3.95 mL, $3.75 \mathrm{~g}, 20.8 \mathrm{mmol}, 1.00$ equiv.) was added over $5 \mathrm{~min}$. The clear solution was stirred at $23{ }^{\circ} \mathrm{C}$ for 14 hours, at which point a white solid had precipitated. The suspension was filtered under $\mathrm{N}_{2}$ via cannula and the yellow filtrate concentrated to a total volume of $10 \mathrm{~mL}$, at which point a white solid precipitated. Addition of water $(2 \mathrm{~mL})$ resulted in a clear biphasic mixture. The organic layer was transferred to a $25 \mathrm{~mL}$ round-bottomed flask and distilled under reduced pressure $(0.25$ Torr, $\left.68^{\circ} \mathrm{C}\right)$ to afford the title compound as a colorless liquid $(4.91 \mathrm{~g}, 51 \%)$.

NMR Spectroscopy: ${ }^{1} \mathrm{H}$ NMR (400 MHz, $\left.\mathrm{C}_{6} \mathrm{D}_{6}, 23{ }^{\circ} \mathrm{C}, \delta\right): 7.66(\mathrm{~d}, J=8.1 \mathrm{~Hz}, 2 \mathrm{H}), 7.08-6.98(\mathrm{~m}, 3 \mathrm{H})$, $2.65(\mathrm{~s}, 3 \mathrm{H}), 1.14\left(\mathrm{~d}, J_{\mathrm{P}-\mathrm{H}}=11.7 \mathrm{~Hz}, 18 \mathrm{H}\right) .{ }^{31} \mathrm{P} \mathrm{NMR}\left(400 \mathrm{MHz}, \mathrm{C}_{6} \mathrm{D}_{6}, 23{ }^{\circ} \mathrm{C}, \delta\right): 16.0$. These spectroscopic data correspond to previously reported data. 


\section{4-Chloro-1-butene (66)}

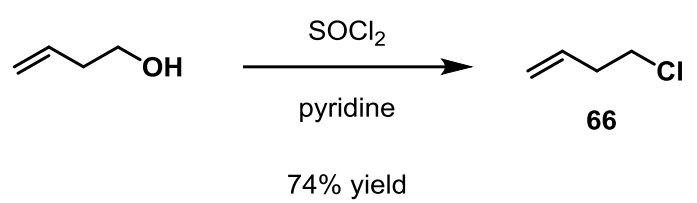

Compound 66 was synthesized by the method of Roberts and Mazur. ${ }^{16}$ Under an atmosphere of dry $\mathrm{N}_{2}$, But-3-ene-1-ol (17.9 mL, 15.0 g, 0.208 mol, 1.00 equiv.), pyridine (0.841 mL, $0.823 \mathrm{~g}, 10.4 \mathrm{mmol}, 5.0$ mol \%), and a Teflon-coated magnetic stirring bar were added to a $100 \mathrm{~mL}$ two-necked flask equipped with a reflux condenser. The flask was chilled in an ice/water bath and thionyl chloride $(15.18 \mathrm{~mL}, 24.75$ g, 0.208 mol, 1.00 equiv.) was added dropwise over $20 \mathrm{~min}$. The resulting yellow solution was stirred cold for $30 \mathrm{~min}$. before heating the solution at reflux for $45 \mathrm{~min}$. The resulting orange biphasic mixture was transferred to a $50 \mathrm{~mL}$ round-bottomed flask under $\mathrm{N}_{2}$ and distilled to yield the title compound as a colorless liquid (13.94 g, 74\%).

NMR Spectroscopy: ${ }^{1} \mathrm{H}$ NMR (400 MHz, $\left.\mathrm{C}_{6} \mathrm{D}_{6}, 23{ }^{\circ} \mathrm{C}, \delta\right): 5.83(\mathrm{~m}, 1 \mathrm{H}), 5.17$ (dq, $J=12.9 \mathrm{~Hz}, J=1.6$ $\mathrm{Hz}, 1 \mathrm{H}), 5.13(\mathrm{dq}, J=6.25 \mathrm{~Hz}, J=1.6 \mathrm{~Hz}), 3.57$ (t, $J=7.0 \mathrm{~Hz} ., 2 \mathrm{H}), 2.53$ (qt, $J=6.8 \mathrm{~Hz}, J=1.5 \mathrm{~Hz}$, 2H). These spectroscopic data correspond to previously reported data.

\section{3-Butenylmagnesium Chloride (67)}

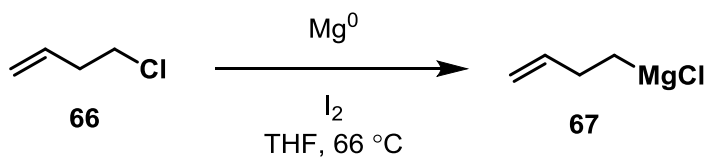

Under an atmosphere of dry $\mathrm{N}_{2}, \mathrm{Mg}$ turnings (4.03 g, $0.166 \mathrm{~mol}, 1.50$ equiv.), THF (20 mL), and a Teflon-coated magnetic stirring bar were added to a $250 \mathrm{~mL}$ 2-neck round-bottomed flask. A portion of 3chloro-1-butene (2.0 g of total: $10.0 \mathrm{~g}, 0.110 \mathrm{mmol}, 1.00$ equiv.) and a few crystals of iodine were added

\footnotetext{
${ }^{16}$ Roberts, J. D.; Mazur, R. H. J. Am. Chem. Soc. 1951, 73, 2509.
} 
as a solid and the mixture was stirred at $23{ }^{\circ} \mathrm{C}$. Once the reaction had initiated, the remainder of the 3 chloro-1-butene was added at a rate that maintained a steady reflux of the reaction mixture. The reaction mixture was diluted with THF (50 mL) and heated to reflux for 14 hours. After cooling the reaction flask at $23{ }^{\circ} \mathrm{C}$, the resulting Grignard solution was filtered by cannula and titrated $(1.15 \mathrm{M})$.

\section{4-Iodo-1-butene (68)}

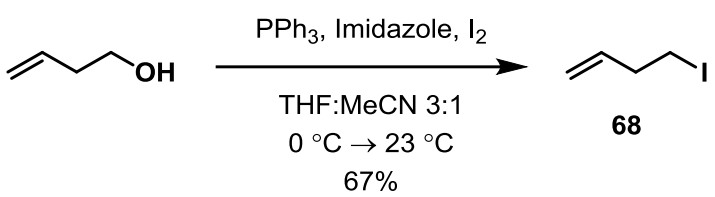

4-Iodo-1-butene was prepared according to the method of Liu and Heathcock. ${ }^{17}$ But-3-en-1-ol (5.90 mL, $4.94 \mathrm{~g}, 68.6 \mathrm{mmol}, 1.00$ equiv.), triphenylphosphine (27.0 g, $103 \mathrm{mmol}, 1.50$ equiv.), imidazole (9.34 g, 137 mmol, 2.0 equiv.), THF (100 mL), MeCN (33 mL) and a Teflon-coated stirring bar were added to a $250 \mathrm{~mL}$ round-bottomed flask to form a clear yellow solution at $23{ }^{\circ} \mathrm{C}$. The solution was chilled at $0{ }^{\circ} \mathrm{C}$ in an ice/water bath and iodine (29.6 g, $117 \mathrm{mmol}, 1.70$ equiv.) was added as a solid, in portions, over 5 min. The solution was allowed to stir for $30 \mathrm{~min}$. at $23{ }^{\circ} \mathrm{C}$. The solution was poured into a separatory funnel containing pentane $\left(150 \mathrm{~mL}\right.$ ) and $0.7 \mathrm{M} \mathrm{Na}_{2} \mathrm{~S}_{2} \mathrm{O}_{3}$ (aq., $150 \mathrm{~mL}$ ). The organic layer was collected and the aqueous layer extracted with pentane $(3 \times 150 \mathrm{~mL})$. The organic layers were combined, washed with saturated $\mathrm{NaCl}$ (aq., $150 \mathrm{~mL}$ ), and dried over $\mathrm{Na}_{2} \mathrm{SO}_{4}$. The clear solution was passed through a silica plug, eluting with pentane $(500 \mathrm{~mL})$, and the resulting solution was concentrated under reduced pressure to afford the product as a red oil $(8.4 \mathrm{~g}, 67 \%)$.

NMR Spectroscopy: ${ }^{1} \mathrm{H}$ NMR (400 MHz, $\left.\mathrm{C}_{6} \mathrm{D}_{6}, 23{ }^{\circ} \mathrm{C}, \delta\right): 5.65-5.52(\mathrm{~m}, 1 \mathrm{H}), 5.08(\mathrm{~d}, J=10.2 \mathrm{~Hz}, 1 \mathrm{H})$, $5.00(\mathrm{~d}, J=17.2 \mathrm{~Hz}, 1 \mathrm{H}), 2.79(\mathrm{t}, J=7.03,2 \mathrm{H}), 2.32(\mathrm{q}, J=6.6 \mathrm{~Hz}, 2 \mathrm{H})$. These spectroscopic data correspond to previously reported data.

${ }^{17}$ Liu, J. F.; Heathcock, C. H. J. Org. Chem. 1999, 64, 8263. 


\section{3-Butenyllithium (69)}

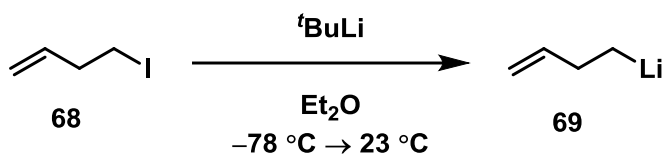

3-Butenyllithium was prepared by the method of Liu and Heathcock. ${ }^{17}$ 4-Iodo-1-butene $(6.11 \mathrm{~g}, 33.6$ mmol, 1.00 equiv.), $\mathrm{Et}_{2} \mathrm{O}(50 \mathrm{~mL})$, and a Teflon-coated stirring bar were added to an oven-dried 2-neck $100 \mathrm{~mL}$ flask under $\mathrm{N}_{2}$. The pale yellow solution was chilled at $-78{ }^{\circ} \mathrm{C}$ in a $\mathrm{CO}_{2} /$ acetone bath, then ${ }^{t} \mathrm{BuLi}$ in pentane (c = $1.5 \mathrm{M}, 44.0 \mathrm{~mL}, 66.0 \mathrm{mmol}, 1.97$ equiv.) was added in portions over $30 \mathrm{~min}$. The solution was stirred at $23{ }^{\circ} \mathrm{C}$ for 14 hours, then concentrated under reduced pressure to a volume of $5 \mathrm{~mL}$. Hexanes $(50 \mathrm{~mL})$ was added and the suspension was stirred for 2 hours at $23{ }^{\circ} \mathrm{C}$, then filtered to afford a clear orange solution of butenyllithium $(\mathrm{c}=1.0 \mathrm{M})$.

NMR Spectroscopy: ${ }^{1} \mathrm{H}$ NMR $\left(400 \mathrm{MHz}, 23{ }^{\circ} \mathrm{C}, \delta\right): 5.78-5.64(\mathrm{~m}, 1 \mathrm{H}), 4.71(\mathrm{~d}, J=17 \mathrm{~Hz}, 1 \mathrm{H}), 4.49(\mathrm{~d}$, $J=10 \mathrm{~Hz}, 1 \mathrm{H}),-1.06(\mathrm{t}, J=8.2 \mathrm{~Hz}, 2 \mathrm{H})$. One signal not observed due to overlap with hexane solvent signals. These spectroscopic data correspond to previously reported data.

\subsection{Optimization of Hydrosilylation Using Precatalysts 16 and 19}

\subsubsection{Comparison of Reductants}

The use of Grignard reagent reductants is necessary for catalysis of hydrosilylation by precatalyst $\mathbf{1 6}$. To elucidate the role of the reductant and especially the counterion, the rate and selectivity of hydrosilylation with 16 is compared using different alkyl metal reductants to activate the precatalyst.

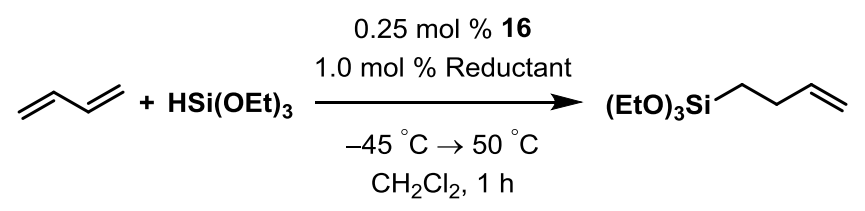




\section{Methyllithium}

In a dry, $\mathrm{N}_{2}$-filled glovebox, platinum precatalyst $16(2.6 \mathrm{mg}, 3.0 \mu \mathrm{mol}, 0.25 \mathrm{~mol} \%)$ and dichloromethane $(0.5 \mathrm{~mL})$ were added to a $4 \mathrm{~mL}$ scintillation vial to form a clear, colorless solution. After chilling at -45 ${ }^{\circ} \mathrm{C}$ in a $\mathrm{CO}_{2} /{ }^{i} \mathrm{PrOH}-$ cooled cold well for 30 min., methyllithium in $\mathrm{Et}_{2} \mathrm{O}(\mathrm{c}=0.76 \mathrm{M}, 16 \mu \mathrm{L}, 12 \mu \mathrm{mol}, 1.0$ mol \%) was added and the reaction tube was sealed and shaken for 90 seconds at $23{ }^{\circ} \mathrm{C}$. The vial was cooled at $-45{ }^{\circ} \mathrm{C}$ for 3 hours, warmed at $23{ }^{\circ} \mathrm{C}$ for $30 \mathrm{~min}$., and cooled at $-45{ }^{\circ} \mathrm{C}$ for $30 \mathrm{~min}$. Butadiene (100. $\mu \mathrm{L}, 64.0 \mathrm{mg}, 1.18 \mathrm{mmol}, 1.00$ equiv.) and triethoxysilane (218 $\mu \mathrm{L}, 194 \mathrm{mg}, 1.18 \mathrm{mmol}, 1.00$ equiv.) were added. The reaction tube was resealed, removed from the glovebox, and heated to $50{ }^{\circ} \mathrm{C}$ in a preheated aluminum heating block for $25 \mathrm{~min}$. The reaction vial was opened under ambient atmosphere and $\mathrm{TMS}_{2} \mathrm{O}(10 \mu \mathrm{L}, 7.6 \mathrm{mg}, 47 \mu \mathrm{mol}, 4.0 \mathrm{~mol} \%)$ and benzene- $d_{6}(100 \mu \mathrm{L})$ were added. The contents of the reaction vial were mixed thoroughly and transferred to an NMR tube. Ratio of 1,2-:1,4-addition determined to be $1.1: 1$ by ${ }^{1} \mathrm{H}$ NMR integration of signals corresponding to alkenyl protons (5.96-5.86 ppm for 3-butenyltriethoxysilane signal corresponds to $1 \mathrm{H}$; 5.49-5.37 for 2-butenyltriethoxysilanes, overlapping signals correspond to $2 \mathrm{H}$ ). Yield determined as $5 \%$ by addition of ${ }^{1} \mathrm{H}$ NMR signal integrations for butenylsilane products (full integration for 3-butenyltriethoxysilane $+1 / 2$ integration for 2 butenyltriethoxysilanes) in comparison to the $\mathrm{TMS}_{2} \mathrm{O}$ signal at $0 \mathrm{ppm}$ (integral set to 71.5 to show integrals as molar percents).

\section{Benzylpotassium}

In a dry, $\mathrm{N}_{2}$-filled glovebox, platinum precatalyst $16(2.6 \mathrm{mg}, 3.0 \mu \mathrm{mol}, 0.25 \mathrm{~mol} \%)$ and benzylpotassium (1.5 mg, $12 \mu \mathrm{mol}, 1.0 \mathrm{~mol} \%$ ) were added to a $4 \mathrm{~mL}$ scintillation vial. After chilling at $-45{ }^{\circ} \mathrm{C}$ in a $\mathrm{CO}_{2} / \mathrm{i} \mathrm{PrOH}$-cooled cold well for $30 \mathrm{~min}$., dichloromethane $(0.5 \mathrm{~mL})$ was added and the reaction tube was sealed and shaken for 90 seconds at $23{ }^{\circ} \mathrm{C}$. The vial was cooled at $-45{ }^{\circ} \mathrm{C}$ for 3 hours, warmed at $23{ }^{\circ} \mathrm{C}$ for $30 \mathrm{~min}$., and cooled at $-45{ }^{\circ} \mathrm{C}$ for $30 \mathrm{~min}$. Butadiene (100. $\mu \mathrm{L}, 64.0 \mathrm{mg}, 1.18 \mathrm{mmol}, 1.00$ equiv.) and triethoxysilane ( $218 \mu \mathrm{L}, 194 \mathrm{mg}, 1.18 \mathrm{mmol}, 1.00$ equiv.) were added. The reaction tube was resealed, 
removed from the glovebox, and heated to $50{ }^{\circ} \mathrm{C}$ in a pre-heated aluminum heating block for $25 \mathrm{~min}$. The reaction vial was opened under ambient atmosphere and $\mathrm{TMS}_{2} \mathrm{O}(10 \mu \mathrm{L}, 7.6 \mathrm{mg}, 47 \mu \mathrm{mol}, 4.0 \mathrm{~mol} \%)$ and benzene- $d_{6}(100 \mu \mathrm{L})$ were added. The contents of the reaction vial were mixed thoroughly and transferred to an NMR tube. Ratio of 1,2-:1,4-addition determined to be 1.3:1 by ${ }^{1} \mathrm{H}$ NMR integration of signals corresponding to alkenyl protons (5.96-5.86 ppm for 3-butenyltriethoxysilane signal corresponds to $1 \mathrm{H} ; 5.49-5.37$ for 2-butenyltriethoxysilanes, overlapping signals correspond to $2 \mathrm{H}$ ). Yield determined as $19 \%$ by addition of ${ }^{1} \mathrm{H}$ NMR signal integrations for butenylsilane products (full integration for 3butenyltriethoxysilane $+1 / 2$ integration for 2-butenyltriethoxysilanes) in comparison to the $\mathrm{TMS}_{2} \mathrm{O}$ signal at $0 \mathrm{ppm}$ (integral set to 71.5 to show integrals as molar percents).

\section{Ethylmagnesium Chloride}

In a dry, $\mathrm{N}_{2}$-filled glovebox, platinum precatalyst $16(2.6 \mathrm{mg}, 3.0 \mu \mathrm{mol}, 0.25 \mathrm{~mol} \%)$ and dichloromethane $(0.5 \mathrm{~mL})$ were added to a $4 \mathrm{~mL}$ scintillation vial to form a clear, colorless solution. After chilling at -45 ${ }^{\circ} \mathrm{C}$ in a $\mathrm{CO}_{2} /{ }^{i} \mathrm{PrOH}-$ cooled cold well for $30 \mathrm{~min}$., ethylmagnesium chloride in THF (c = 2.0 M, $5.9 \mu \mathrm{L}, 12$ $\mu \mathrm{mol}, 1.0 \mathrm{~mol} \%$ ) was added and the reaction tube was sealed and shaken for 90 seconds at $23{ }^{\circ} \mathrm{C}$ to dissolve frozen droplets of the Grignard solution. The vial was cooled at $-45{ }^{\circ} \mathrm{C}$ for 3 hours, warmed at $23{ }^{\circ} \mathrm{C}$ for $30 \mathrm{~min}$., and cooled at $-45{ }^{\circ} \mathrm{C}$ for $30 \mathrm{~min}$. Butadiene (100. $\mu \mathrm{L}, 64.0 \mathrm{mg}, 1.18 \mathrm{mmol}, 1.00$ equiv.) and triethoxysilane ( $218 \mu \mathrm{L}, 194 \mathrm{mg}, 1.18 \mathrm{mmol}, 1.00$ equiv.) were added. The reaction tube was resealed, removed from the glovebox, and heated to $50{ }^{\circ} \mathrm{C}$ in a pre-heated aluminum heating block for 25 min. The reaction vial was opened under ambient atmosphere and $\mathrm{TMS}_{2} \mathrm{O}(10 \mu \mathrm{L}, 7.6 \mathrm{mg}, 47 \mu \mathrm{mol}, 4.0$ mol $\%)$ and benzene- $d_{6}(100 \mu \mathrm{L})$ were added. The contents of the reaction vial were mixed thoroughly and transferred to an NMR tube. Ratio of 1,2-:1,4-addition determined to be 9:1 by ${ }^{1} \mathrm{H}$ NMR integration of signals corresponding to alkenyl protons (5.96-5.86 ppm for 3-butenyltriethoxysilane signal corresponds to $1 \mathrm{H} ; 5.49-5.37$ for 2-butenyltriethoxysilanes, overlapping signals correspond to $2 \mathrm{H}$ ). Yield determined as $57 \%$ by addition of ${ }^{1} \mathrm{H}$ NMR signal integrations for butenylsilane products (full integration 
for 3-butenyltriethoxysilane $+1 / 2$ integration for 2-butenyltriethoxysilanes) in comparison to the $\mathrm{TMS}_{2} \mathrm{O}$ signal at $0 \mathrm{ppm}$ (integral set to 71.5 to show integrals as molar percents).

\section{Phenylmagnesium Chloride}

In a dry, $\mathrm{N}_{2}$-filled glovebox, platinum precatalyst $16(2.6 \mathrm{mg}, 3.0 \mu \mathrm{mol}, 0.25 \mathrm{~mol} \%)$ and dichloromethane $(0.5 \mathrm{~mL})$ were added to a $4 \mathrm{~mL}$ scintillation vial to form a clear, colorless solution. After chilling at -45 ${ }^{\circ} \mathrm{C}$ in a $\mathrm{CO}_{2} / \mathrm{PrOH}$-cooled cold well for $30 \mathrm{~min}$., phenylmagnesium chloride in $\mathrm{THF}(\mathrm{c}=2.0 \mathrm{M}, 5.9 \mu \mathrm{L}$, $12 \mu \mathrm{mol}, 1.0 \mathrm{~mol} \%$ ) was added and the reaction tube was sealed and shaken for 90 seconds at $23{ }^{\circ} \mathrm{C}$ to dissolve frozen droplets of the Grignard solution. The vial was cooled at $-45{ }^{\circ} \mathrm{C}$ for 3 hours, warmed at $23{ }^{\circ} \mathrm{C}$ for $30 \mathrm{~min}$., and cooled at $-45{ }^{\circ} \mathrm{C}$ for $30 \mathrm{~min}$. Butadiene $(100 . \mu \mathrm{L}, 64.0 \mathrm{mg}, 1.18 \mathrm{mmol}, 1.00$ equiv.) and triethoxysilane (218 $\mu \mathrm{L}, 194 \mathrm{mg}, 1.18 \mathrm{mmol}, 1.00$ equiv.) were added. The reaction tube was resealed, removed from the glovebox, and heated to $50{ }^{\circ} \mathrm{C}$ in a pre-heated aluminum heating block for 25 min. The reaction vial was opened under ambient atmosphere and $\mathrm{TMS}_{2} \mathrm{O}(10 \mu \mathrm{L}, 7.6 \mathrm{mg}, 47 \mu \mathrm{mol}, 4.0$ mol \%) and benzene- $d_{6}(100 \mu \mathrm{L})$ were added. The contents of the reaction vial were mixed thoroughly and transferred to an NMR tube. Ratio of 1,2-:1,4-addition determined to be 9.4:1 by ${ }^{1} \mathrm{H}$ NMR integration of signals corresponding to alkenyl protons (5.96-5.86 ppm for 3-butenyltriethoxysilane signal corresponds to $1 \mathrm{H} ; 5.49-5.37$ for 2-butenyltriethoxysilanes, overlapping signals correspond to $2 \mathrm{H}$ ). Yield determined as $55 \%$ by addition of ${ }^{1} \mathrm{H}$ NMR signal integrations for butenylsilane products (full integration for 3-butenyltriethoxysilane $+1 / 2$ integration for 2-butenyltriethoxysilanes) in comparison to the $\mathrm{TMS}_{2} \mathrm{O}$ signal at $0 \mathrm{ppm}$ (integral set to 71.5 to show integrals as molar percents).

\subsubsection{Comparison of Solvents}

Activation of precatalyst $\mathbf{1 6}$ by Grignard reagents requires optimization for efficient activation in solvents other than dichloromethane. To probe the response of hydrosilylation yield and selectivity to different solvents, we have used precatalyst $\mathbf{1 9}$ which has already been partially activated. 


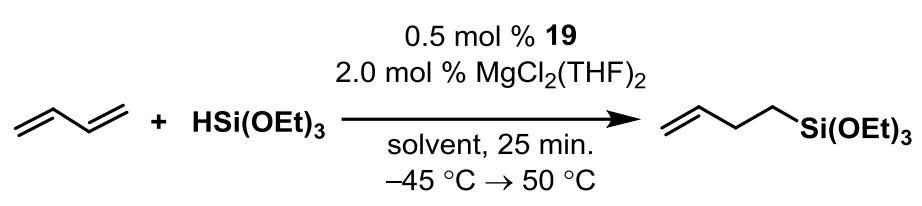

\section{General Procedure}

In a dry, $\mathrm{N}_{2}$-filled glovebox, platinum precatalyst 19 (3.9 mg, $\left.5.9 \mu \mathrm{mol}, 0.5 \mathrm{~mol} \%\right), \mathrm{MgCl}_{2}(\mathrm{THF})_{2}(5.6$ $\mathrm{mg}, 24 \mu \mathrm{mol}, 2.0 \mathrm{~mol} \%)$, solvent $(0.5 \mathrm{~mL})$, and tetrahydrofuran $(10 \mu \mathrm{L})$ were added to a $4 \mathrm{~mL}$ scintillation vial at $23{ }^{\circ} \mathrm{C}$. The vial was chilled at $-45{ }^{\circ} \mathrm{C}$ in a $\mathrm{CO}_{2} / i \mathrm{PrOH}$-cooled cold well for 30 min. Butadiene (100. $\mu \mathrm{L}, 64.0 \mathrm{mg}, 1.18 \mathrm{mmol}, 1.00$ equiv.) and triethoxysilane (218 $\mu \mathrm{L}, 194 \mathrm{mg}, 1.18 \mathrm{mmol}$, 1.00 equiv.) were added and the reaction vial was sealed with a Teflon-lined cap, removed from the glovebox, and heated at $50{ }^{\circ} \mathrm{C}$ in a pre-heated aluminum heating block for $25 \mathrm{~min}$. The reaction vial was opened under ambient atmosphere and $\mathrm{TMS}_{2} \mathrm{O}(10 \mu \mathrm{L}, 47 \mu \mathrm{mol}, 4.0 \mathrm{~mol} \%)$ and benzene- $d_{6}(100 \mu \mathrm{L})$ were added. The contents of the reaction vial were mixed thoroughly and transferred to an NMR tube. Ratio of 1,2-:1,4-addition determined by ${ }^{1} \mathrm{H}$ NMR integration of signals corresponding to alkenyl protons (5.96-5.86 ppm for 3-butenyltriethoxysilane signal corresponds to $1 \mathrm{H} ; \quad 5.49-5.37$ for 2butenyltriethoxysilanes, overlapping signals correspond to $2 \mathrm{H}$ ). Yield determined by addition of ${ }^{1} \mathrm{H}$ NMR signal integrations for butenylsilane products (full integration for 3-butenyltriethoxysilane $+1 / 2$ integration for 2-butenyltriethoxysilanes) in comparison to the $\mathrm{TMS}_{2} \mathrm{O}$ signal at $0 \mathrm{ppm}$ (integral set to 71.5 to show integrals as molar percents). 
Table 15. Solvent Optimization

\begin{tabular}{ccc} 
Solvent & Yield & $\mathbf{1 , 2 - : 1 , 4 - a d d i t i o n ~}$ \\
\hline Dichloromethane & $86 \%$ & $10: 1$ \\
\hline Tetrahydrofuran & $9 \%$ & $2: 1$ \\
\hline Diethyl Ether & $31 \%$ & $6: 1$ \\
\hline Acetonitrile & $6 \%$ & $6: 1$ \\
\hline Benzonitrile & $28 \%$ & $8: 1$ \\
\hline Benzene & $43 \%$ & $7: 1$ \\
\hline Toluene & $37 \%$ & $5: 1$ \\
\hline Pentane & $9 \%$ & $3: 1$
\end{tabular}

\subsubsection{Comparison of Silanes}

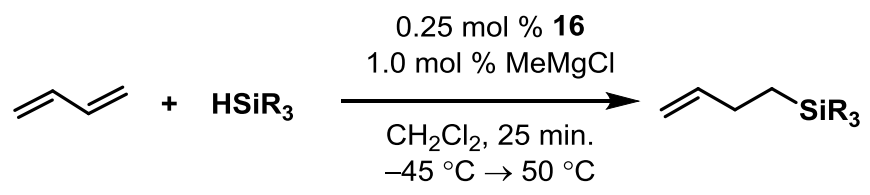

\section{General Procedure}

In a dry, $\mathrm{N}_{2}$-filled glovebox, platinum precatalyst $16(2.6 \mathrm{mg}, 3.0 \mu \mathrm{mol}, 0.25 \mathrm{~mol} \%)$ and dichloromethane $(0.5 \mathrm{~mL})$ were added to a $4 \mathrm{~mL}$ scintillation vial to form a clear, colorless solution. After chilling at -45 ${ }^{\circ} \mathrm{C}$ in a $\mathrm{CO}_{2} /{ }^{i} \mathrm{PrOH}$-cooled cold well for 30 min., methylmagnesium chloride in THF $(\mathrm{c}=3.2 \mathrm{M}, 3.6 \mu \mathrm{L}$, $12 \mu \mathrm{mol}, 1.0 \mathrm{~mol} \%$ ) was added and the reaction tube was sealed and shaken for 90 seconds at $23{ }^{\circ} \mathrm{C}$. The vial was cooled at $-45^{\circ} \mathrm{C}$ for 3 hours, warmed at $23{ }^{\circ} \mathrm{C}$ for $30 \mathrm{~min}$., and cooled at $-45{ }^{\circ} \mathrm{C}$ for $30 \mathrm{~min}$. Butadiene (100. $\mu \mathrm{L}, 64.0 \mathrm{mg}, 1.18 \mathrm{mmol}, 1.00$ equiv.) and silane (1.00 equiv.) were added. The reaction tube was resealed, removed from the glovebox, and heated to $50{ }^{\circ} \mathrm{C}$ in a pre-heated aluminum heating block for $25 \mathrm{~min}$. The reaction vial was opened under ambient atmosphere and $\mathrm{TMS}_{2} \mathrm{O}(10 \mu \mathrm{L}, 7.6 \mathrm{mg}$, $47 \mu \mathrm{mol}, 4.0 \mathrm{~mol} \%)$ and benzene- $d_{6}(100 \mu \mathrm{L})$ were added. The contents of the reaction vial were mixed 
thoroughly and transferred to an NMR tube. Ratio of 1,2-:1,4-addition determined to be $1.1: 1$ by ${ }^{1} \mathrm{H}$ NMR integration of signals corresponding to alkenyl protons $(5.96-5.86 \mathrm{ppm}$ for 3-butenylsilane signal corresponds to $1 \mathrm{H} ; 5.49-5.37$ for 2-butenylsilanes, overlapping signals correspond to $2 \mathrm{H}$ ). Yield determined as $5 \%$ by addition of ${ }^{1} \mathrm{H}$ NMR signal integrations for butenylsilane products (full integration for 3-butenylsilane $+1 / 2$ integration for 2-butenylsilanes) in comparison to the $\mathrm{TMS}_{2} \mathrm{O}$ signal at $0 \mathrm{ppm}$ (integral set to 71.5 to show integrals as molar percents).

Table 16. Comparison of Silanes

\begin{tabular}{ccc} 
Silane & Yield & $\mathbf{1 , 2 - : 1 , 4 - a d d i t i o n}$ \\
\hline Triethoxysilane & $86 \%$ & $10: 1$ \\
\hline Triethylsilane & $2 \%$ & $0.3: 1$ \\
\hline Dimethylphenylsilane & $10 \%$ & $0.4: 1$ \\
\hline Trichlorosilane & $19 \%$ & $0.5: 1$
\end{tabular}

\subsubsection{Room Temperature Reaction Initiation}

Low-temperature activation of precatalyst 16, although effective and manageable on a laboratory scale, cannot be effectively used in industrial reactions. Activation of precatalyst $\mathbf{1 6}$ requires low temperatures, but pre-formed platinates 19 and 32 should not exhibit dependence on reaction initiation temperature as they do not require activation by Grignard reagents. In this experiment, we initiate hydrosilylation at 23 ${ }^{\circ} \mathrm{C}$ using precatalyst $\mathbf{1 9}$ to show that the reaction outcome is the same as reactions initiated at low temperature.

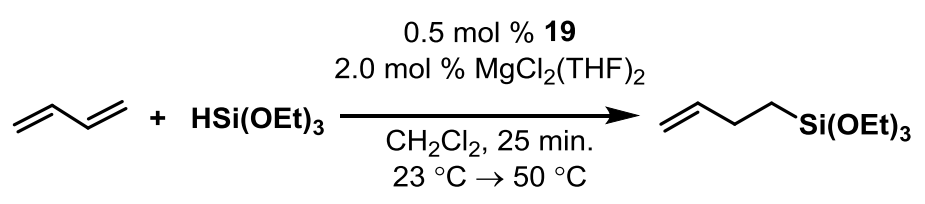




\section{Procedure for hydrosilylation starting at $23^{\circ} \mathrm{C}$}

In a dry, $\mathrm{N}_{2}$-filled glovebox, platinum precatalyst $19(3.9 \mathrm{mg}, 5.9 \mu \mathrm{mol}, 0.5 \mathrm{~mol} \%)$, dichloromethane $(0.5$ $\mathrm{mL})$, and tetrahydrofuran $(10 \mu \mathrm{L})$ were added to a $4 \mathrm{~mL}$ scintillation vial at $23{ }^{\circ} \mathrm{C}$. A second $4 \mathrm{~mL}$ scintillation vial was charged with $\mathrm{MgCl}_{2}(\mathrm{THF})_{2}(5.6 \mathrm{mg}, 24 \mu \mathrm{mol}, 2.0 \mathrm{~mol} \%$ ) and a Teflon-coated stirring bar and chilled at $-45^{\circ} \mathrm{C}$ in a $\mathrm{CO}_{2} /{ }^{i} \mathrm{PrOH}-$ cooled cold well for $15 \mathrm{~min}$. Butadiene $(100 . \mu \mathrm{L}, 64.0$ mg, 1.18 mmol, 1.00 equiv.) and triethoxysilane (218 $\mu \mathrm{L}, 194 \mathrm{mg}, 1.18 \mathrm{mmol}, 1.00$ equiv.) were added to the vial containing $\mathrm{MgCl}_{2}$ (THF) $)_{2}$ and this vial was sealed with a mininert cap and allowed to warm at 23 ${ }^{\circ} \mathrm{C}$ for 15 min. with the mininert cap in the "closed" position. The solution of precatalyst 6 was added quickly to the suspension of $\mathrm{MgCl}_{2}(\mathrm{THF})_{2}$ in the following manner: the solution of precatalyst 6 was taken up into a $1 \mathrm{~mL}$ syringe, the mininert cap on the Mg-containing vial was switched to the "open" position, the contents of the syringe were added to the Mg-containing vial, and the mininert cap was resealed in the "closed" position. The reaction vial was removed from the glovebox and heated at $50{ }^{\circ} \mathrm{C}$ in a pre-heated aluminum heating block for $25 \mathrm{~min}$. The reaction vial was opened under ambient atmosphere and $\mathrm{TMS}_{2} \mathrm{O}(10 \mu \mathrm{L}, 47 \mu \mathrm{mol}, 4.0 \mathrm{~mol} \%)$ and benzene- $d_{6}(100 \mu \mathrm{L})$ were added. The contents of the reaction vial were mixed thoroughly and transferred to an NMR tube. Ratio of 1,2-:1,4-addition determined to be $9: 1$ by ${ }^{1} \mathrm{H}$ NMR integration of signals corresponding to alkenyl protons (5.96-5.86 ppm for 3-butenyltriethoxysilane signal corresponds to $1 \mathrm{H} ; 5.49-5.37$ for 2-butenyltriethoxysilanes, overlapping signals correspond to $2 \mathrm{H}$ ). Yield determined to be $71.7 \%$ by addition of ${ }^{1} \mathrm{H}$ NMR signal integrations for butenylsilane products (full integration for 3-butenyltriethoxysilane $+1 / 2$ integration for 2 butenyltriethoxysilanes) in comparison to the $\mathrm{TMS}_{2} \mathrm{O}$ signal at $0 \mathrm{ppm}$ (integral set to 71.5 to show integrals as molar percents). 


\subsection{Mechanism Elucidation}

\subsubsection{Reaction of 32 with Oxidants}

\section{General Procedure}

In a dry, $\mathrm{N}_{2}$-filled glovebox, platinum precatalyst $16(10.0 \mathrm{mg}, 12.0 \mu \mathrm{mol}, 1.0$ equiv.) and dichloromethane- $d_{2}(0.5 \mathrm{~mL})$ were added to a screw-cap NMR tube to form a clear, colorless solution. After chilling at $-45{ }^{\circ} \mathrm{C}$ in a $\mathrm{CO}_{2} /{ }^{i} \mathrm{PrOH}$-cooled cold well for 30 min., methylmagnesium chloride in THF (c $=3.0 \mathrm{M}, 16 \mu \mathrm{L}, 46 \mu \mathrm{mol}, 4.0$ equiv.) was added and the reaction tube was sealed and shaken for 90 seconds at $23{ }^{\circ} \mathrm{C}$ to dissolve frozen droplets of Grignard reagent. The tube was cooled at $-45{ }^{\circ} \mathrm{C}$ for 3 hours, warmed at $23{ }^{\circ} \mathrm{C}$ for $30 \mathrm{~min}$., and cooled at $-45{ }^{\circ} \mathrm{C}$ for $30 \mathrm{~min}$. Butadiene $(10 \mu \mathrm{L})$ and oxidant $(2.0$ equiv.) were added and the tube was sealed with a Teflon-lined cap. The reaction tube was removed from the glovebox and heated to $50{ }^{\circ} \mathrm{C}$ in a $400 \mathrm{MHz}$ NMR spectrometer. ${ }^{31} \mathrm{P}$ and ${ }^{1} \mathrm{H}$ NMR spectra were collected to determine the reaction outcome.

Table 17. Reactions of Platinum Complex 32 with Oxidants

\begin{tabular}{cc} 
Oxidant & Products \\
\hline $\mathrm{HSi}(\mathrm{OEt})_{3}$ & $\mathbf{1 9}$ \\
\hline $\mathrm{HSiEt}_{3}$ & $\mathbf{3 2}($ no reaction $)$ \\
\hline $\mathrm{HSiPhMe}_{2}$ & $\mathbf{3 2}($ no reaction $)$ \\
\hline $\mathrm{HSiPh}_{3}$ & $\mathbf{3 2}($ no reaction $)$ \\
\hline $\mathrm{HSiCl}_{3}$ & $\mathrm{P}\left({ }^{t} \mathrm{Bu}\right)_{3}($ decomposition $)$ \\
\hline$(\mathrm{PinB})_{2}$ & $\begin{array}{c}\mathrm{P}\left({ }^{t} \mathrm{Bu}\right)_{3}+\mathbf{3 2} \\
(\text { partial decomposition })\end{array}$
\end{tabular}

\subsubsection{Observation of Catalyst/Ligand Products after Hydrosilylation}

In order to support our hypothesis regarding the decomposition pathways prevalent for precatalysts $\mathbf{1}$ and 5-8, we observed the ${ }^{31} \mathrm{P}$ NMR spectrum of hydrosilylation using precatalyst 1 after the reaction was complete. Because the ligand is the only ${ }^{31} \mathrm{P}$-containing species in the reaction, we can reasonably assume 
that signals observed in the ${ }^{31} \mathrm{P}$ NMR spectrum are derived from the ligand of the precatalyst used in hydrosilylation. We have identified the major product in the ${ }^{31} \mathrm{P}$ NMR spectrum as $\mathrm{P}\left({ }^{t} \mathrm{Bu}\right)_{3}$ by spiking in an authentic sample of $\mathrm{P}\left({ }^{t} \mathrm{Bu}\right)_{3}$ after the initial analysis and observing that no new signals are present in the ${ }^{31} \mathrm{P}$ NMR spectrum.

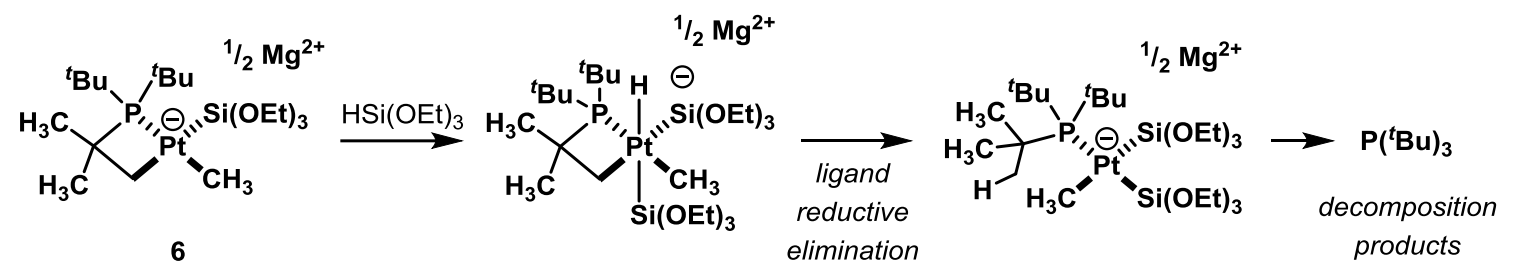

Scheme 28. Proposed Catalyst Decomposition Pathway With Formation of $\mathrm{P}\left({ }^{t} \mathrm{Bu}\right)_{3}$

\section{Procedure:}

In a dry, $\mathrm{N}_{2}$-filled glovebox, platinum precatalyst $1(3.1 \mathrm{mg}, 3.6 \mu \mathrm{mol}, 0.30 \mathrm{~mol} \%)$ and dichloromethane$d_{2}(0.5 \mathrm{~mL})$ were added to a J. Young NMR tube to form a clear, colorless solution. After chilling at -45 ${ }^{\circ} \mathrm{C}$ in a $\mathrm{CO}_{2} /{ }^{i} \mathrm{PrOH}-$ cooled cold well for $30 \mathrm{~min}$., methylmagnesium chloride in THF $(\mathrm{c}=3.3 \mathrm{M}, 4.3 \mu \mathrm{L}$, $14 \mu \mathrm{mol}, 1.2 \mathrm{~mol} \%$ ) was added and the reaction tube was sealed and shaken for 90 seconds at $23{ }^{\circ} \mathrm{C}$ to dissolve frozen droplets of Grignard reagent. The tube was cooled at $-45{ }^{\circ} \mathrm{C}$ for 3 hours, warmed at $23{ }^{\circ} \mathrm{C}$ for $30 \mathrm{~min}$., and cooled at $-45{ }^{\circ} \mathrm{C}$ for $30 \mathrm{~min}$. Butadiene (100. $\mu \mathrm{L}, 64.0 \mathrm{mg}, 1.18 \mathrm{mmol}, 1.00$ equiv.) and triethoxysilane ( $218 \mu \mathrm{L}, 194 \mathrm{mg}, 1.18 \mathrm{mmol}, 1.00$ equiv.) were added. The reaction tube was resealed, removed from the glovebox, and heated to $50{ }^{\circ} \mathrm{C}$ in a $400 \mathrm{MHz}$ NMR spectrometer for $25 \mathrm{~min}$. The ${ }^{31} \mathrm{P}$ NMR spectrum of the reaction tube was recorded, showing one tall peak at $65 \mathrm{ppm}$ and one much shorter peak at $73 \mathrm{ppm}$. The reaction tube was cooled at $23{ }^{\circ} \mathrm{C}$ and brought into the glovebox. Tri-tertbutylphosphine was added $(5.0 \mathrm{mg})$ and the reaction tube was resealed, brought out of the glovebox, and the ${ }^{31} \mathrm{P}$ NMR spectrum was measured again, showing no additional signals, but an increase in relative size of the peak at $65 \mathrm{ppm}$. 


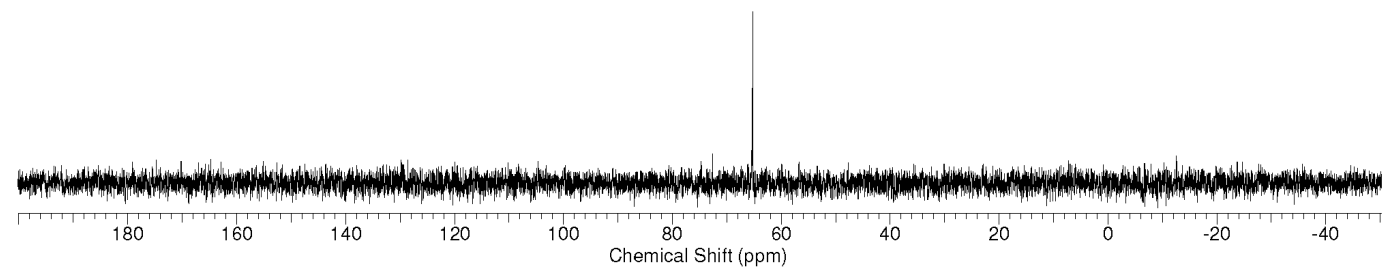

Figure 8. ${ }^{31} \mathrm{P}$ NMR Spectrum of Reaction Products

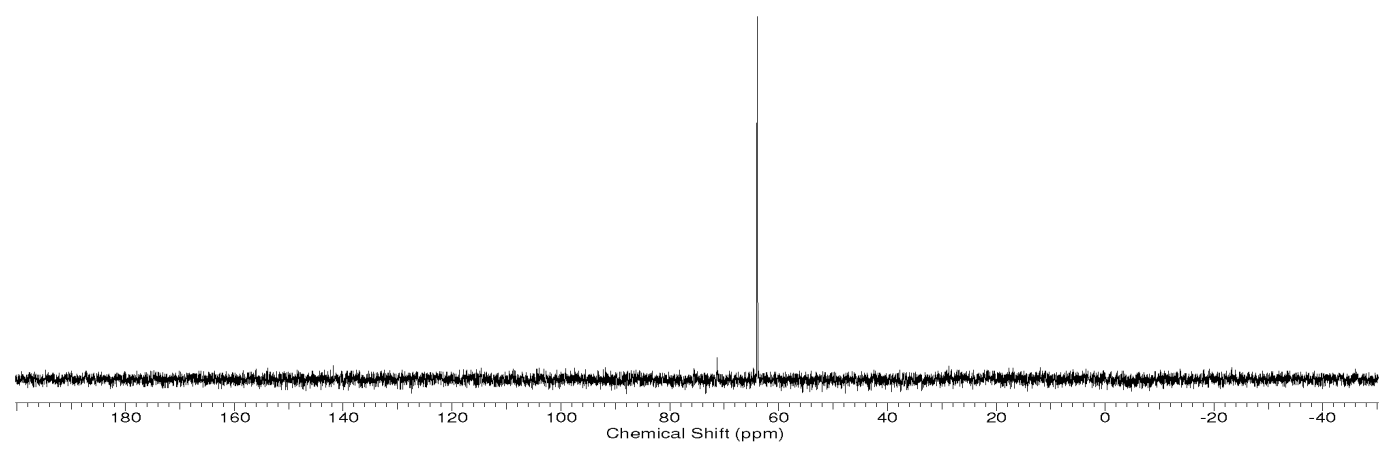

Figure 9. ${ }^{31} \mathrm{P}$ NMR Spectrum of Reaction Products $+\mathrm{P}\left({ }^{\mathrm{t}} \mathrm{Bu}\right)_{3}$

\subsubsection{Detection of Methane During Catalyst Activation}

In the activation pathway of precatalyst $\mathbf{1 9}$ for catalysis we propose oxidative addition of triethoxysilane followed by reductive elimination of methane to form the active catalyst (A). To provide support for this activation mechanism, we mixed $\mathbf{1 9}$ with triethoxysilane and sampled the headspace of the reaction to analyze the methane content by GCMS. 
Because gases cannot be easily separated by the GCMS instrument available to us, all gases present in the headspace elute as a single peak. Such gases typically include $\mathrm{N}_{2}, \mathrm{O}_{2}, \mathrm{H}_{2} \mathrm{O}$, and other naturally occurring atmospheric gases. The quantity of a single component can be analyzed by integrating the spectrum observed at a single $\mathrm{m} / \mathrm{z}$ value. For methane, we measure the intensity of the $\mathrm{m} / \mathrm{z}=16$ peak in the GCMS spectrum. Oxygen can also give rise to $\mathrm{m} / \mathrm{z}=16$ when ionized to $\mathrm{O}_{2}{ }^{2+}$, but this ionization state is much less abundant than $\mathrm{O}_{2}{ }^{+}$, which shows $\mathrm{m} / \mathrm{z}=32$. Methane can be detected by comparing the integration of $\mathrm{m} / \mathrm{z}=16$ to $\mathrm{m} / \mathrm{z}=32$ in the sample compared to a background atmospheric measurement. If methane is present, an enrichment in $\mathrm{m} / \mathrm{z}=16$ in the headspace of the reaction compared to that of naturally occurring atmospheric $\mathrm{O}_{2}$ is observed.

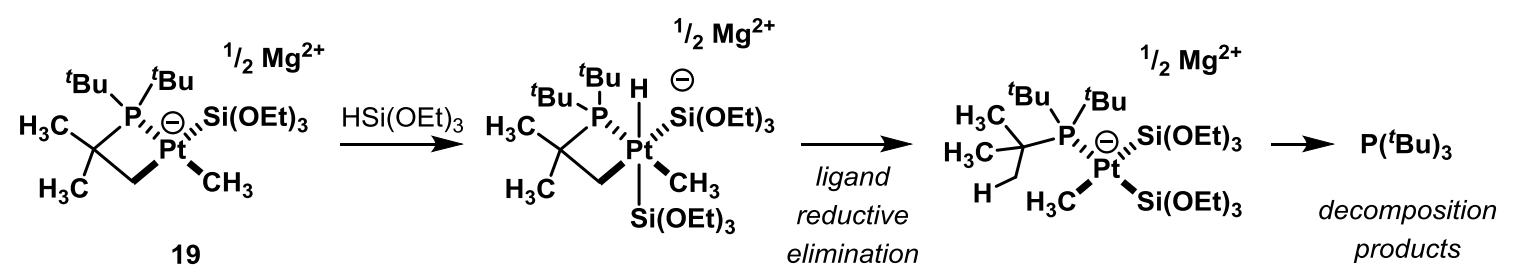

Scheme 29. Proposed Synthesis of Active Catalyst A with Release of Methane

\section{Background Spectrum Analysis}

In a dry, $\mathrm{N}_{2}$-filled glovebox a $4 \mathrm{~mL}$ scintillation vial was sealed with a cap containing a Teflon-lined rubber septum. The vial was removed from the glovebox and a sample of the gas from this vial was analyzed by GCMS (m/z 16/32 = 0.051).

\section{Reaction Headspace Analysis}

In a dry, $\mathrm{N}_{2}$-filled glovebox precatalyst 19 (22.5 mg, $26.0 \mu \mathrm{mol}, 1.00$ equiv.), a Teflon-coated magnetic stirring bar, and dichloromethane- $d_{2}(0.5 \mathrm{~mL})$ were added to a $4 \mathrm{~mL}$ scintillation vial to form a clear, pale yellow solution. The reaction vial was cooled for $30 \mathrm{~min}$. at $-45{ }^{\circ} \mathrm{C}$ in a $\mathrm{CO}_{2} /{ }^{i} \mathrm{PrOH}$-cooled cold well before adding triethoxysilane ( $20 \mu \mathrm{L}, 18 \mathrm{mg}, 0.11 \mathrm{mmol}, 4.1$ equiv.). The reaction vial was sealed with a mininert cap containing a butyl rubber septum, removed from the glovebox, and heated at $50{ }^{\circ} \mathrm{C}$ in a pre- 
heated aluminum heating block for $45 \mathrm{~min}$. After cooling the reaction vial at $23{ }^{\circ} \mathrm{C}$ for $30 \mathrm{~min}$., a $50 \mu \mathrm{L}$ gastight syringe was flushed with the headspace atmosphere three times before $3.5 \mu \mathrm{L}$ of gas from the headspace was analyzed by GCMS (m/z 16/32=0.94).

\section{Table 18. Detection of $\mathrm{CH}_{4}$ by GCMS}

\begin{tabular}{cc} 
Sample & $\mathbf{m} / \mathbf{z}=\mathbf{1 6} / \mathbf{3 2}$ \\
\hline Headspace of $\mathbf{1 9}+\mathrm{HSi}(\mathrm{OEt})_{3}$ & 0.94 \\
\hline $\mathrm{N}_{2}($ Control $)$ & 0.051
\end{tabular}

\subsubsection{Test of Butenylsilane Reductive Elimination from Precatalyst 36}

To further support our claim that precatalyst $\mathbf{3 6}$ is not an intermediate on the catalytic cycle, we tested precatalyst $\mathbf{3 6}$ to see if reductive elimination of butenylsilane is facile under conditions that mimic those present during hydrosilylation. Although some decomposition of precatalyst $\mathbf{3 6}$ was observed, the bulk of the material was stable in solution at $50{ }^{\circ} \mathrm{C}$, indicating that the rate of reductive elimination from $\mathbf{3 6}$ is much slower than the rate of catalysis. From these data, as well as those presented for precatalyst $\mathbf{3 6}$ in hydrosilylation in Table S1, we conclude that precatalyst $\mathbf{3 6}$ cannot be an intermediate on the catalytic cycle.

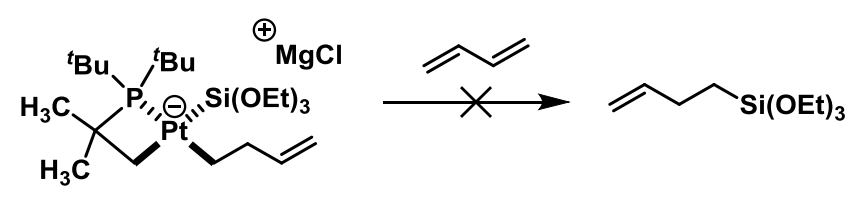

In a dry, $\mathrm{N}_{2}$-filled glovebox, platinum complex $42(10.2 \mathrm{mg}, 17.0 \mu \mathrm{mol}, 1.0$ equiv.) was added to a $4 \mathrm{~mL}$ scintillation vial and chilled for $30 \mathrm{~min}$. at $-45{ }^{\circ} \mathrm{C}$ in a $\mathrm{CO}_{2}{ }^{i} \mathrm{PrOH}-$ cooled cold well. Pre-cooled dichloromethane- $d_{2}(0.5 \mathrm{~mL})$ was added to the vial to form a pale yellow solution. But-3-enylmagnesium chloride in THF ( $\mathrm{c}=1.36 \mathrm{M}, 13 \mu \mathrm{L}, 18 \mu \mathrm{mol}, 1.04$ equiv.) was added and the vial sealed with a Teflonlined cap and shaken for 90 seconds at $23{ }^{\circ} \mathrm{C}$ to dissolve frozen droplets of Grignard reagent. The solution 
was stirred for $30 \mathrm{~min}$ at $-45^{\circ} \mathrm{C}$, at $23{ }^{\circ} \mathrm{C}$ for $30 \mathrm{~min}$., then cooled at $-45^{\circ} \mathrm{C}$ for $30 \mathrm{~min}$. Butadiene was added $(20 \mu \mathrm{L})$ and the solution was transferred to a pre-cooled NMR tube which was sealed with a Teflon-lined cap and removed from the glovebox. The NMR tube was heated to $50{ }^{\circ} \mathrm{C}$ in a $400 \mathrm{MHz}$ NMR spectrometer and the ${ }^{31} \mathrm{P}$ NMR spectrum was measured after 5 min. showing precatalyst $\mathbf{3 6}$ as the major species.

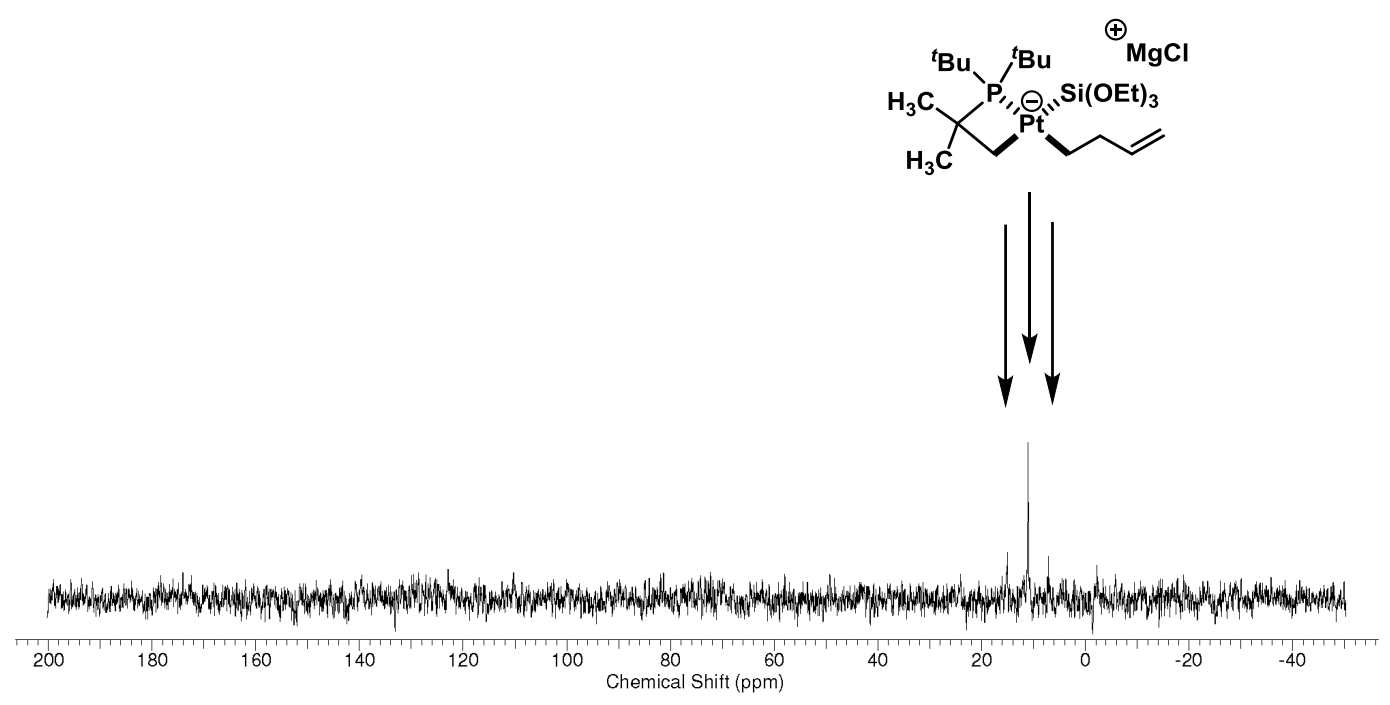

Figure 10. Room temperature ${ }^{31} \mathrm{P}$ NMR Spectrum of $\mathbf{3 6}+$ butadiene: 

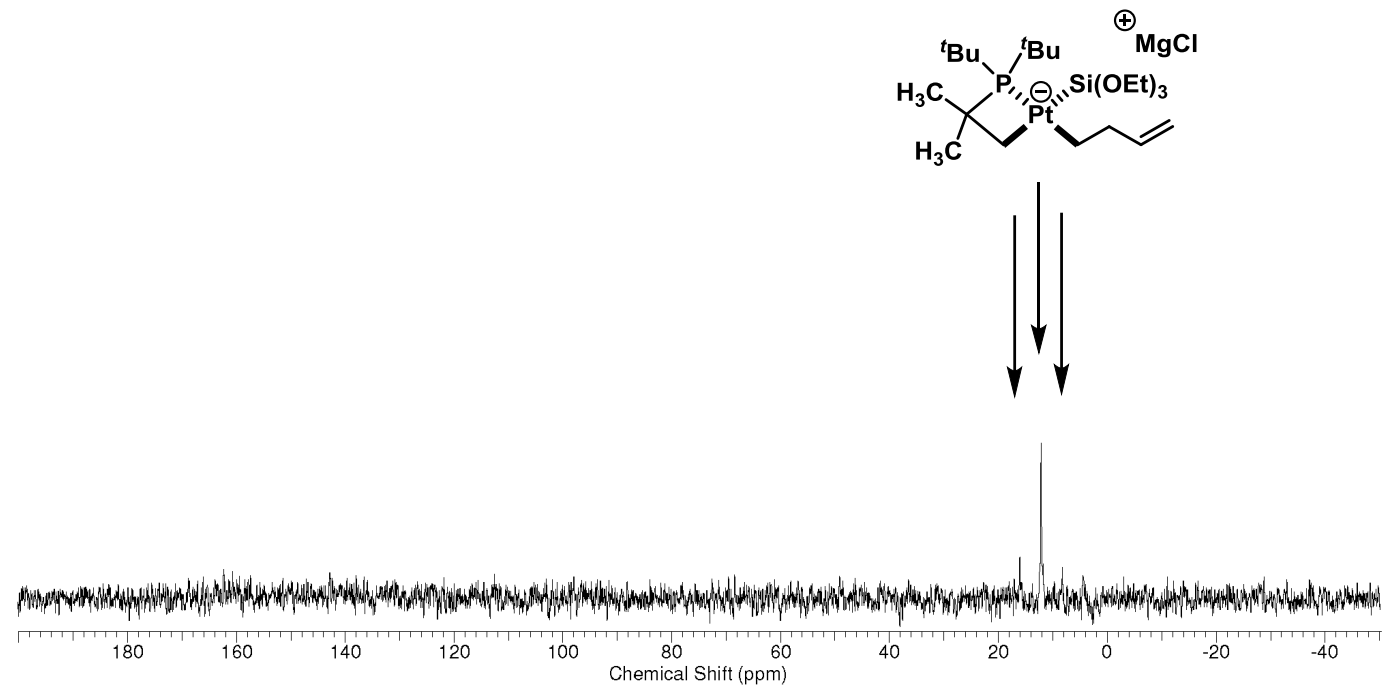

Figure 11. Spectrum of $\mathbf{3 6}+$ butadiene after 5 minutes at $50{ }^{\circ} \mathrm{C}$

\subsubsection{Comparison of Platinum Pre-Catalysts}

Because precatalysts 32, 19, 37, and 36 exist as aggregates with $\mathrm{MgCl}_{2}(\mathrm{THF})_{2}$, the exact catalyst loading for the following experiments cannot be precisely calculated. For the purpose of these experiments, we assumed the smallest possible molecular weight for each complex (calculated with $0.5 \mathrm{Mg}^{2+}$ as the counter ion and the number of THF molecules observed in the ${ }^{1} \mathrm{H}$ NMR spectrum of the isolated complex). As a consequence, the catalyst loadings reported below correspond to the upper limit of possible catalyst loadings.

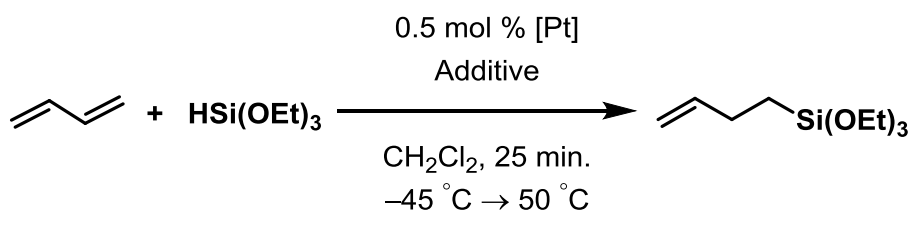

\section{Precatalyst 16}

In a dry, $\mathrm{N}_{2}$-filled glovebox, platinum precatalyst $16(2.6 \mathrm{mg}, 3.0 \mu \mathrm{mol}, 0.25 \mathrm{~mol} \%)$ and dichloromethane- $d_{2}(0.5 \mathrm{~mL})$ were added to a J. Young NMR tube to form a clear, colorless solution. 
After chilling at $-45{ }^{\circ} \mathrm{C}$ in a $\mathrm{CO}_{2} /{ }^{i} \mathrm{PrOH}$-cooled cold well for 30 min., methylmagnesium chloride in $\mathrm{THF}$ $(\mathrm{c}=3.28 \mathrm{M}, 3.6 \mu \mathrm{L}, 10 \mu \mathrm{mol}, 1.0 \mathrm{~mol} \%$ ) was added and the reaction tube was sealed and shaken for 90 seconds at $23{ }^{\circ} \mathrm{C}$ to dissolve frozen droplets of Grignard reagent. The tube was cooled at $-45{ }^{\circ} \mathrm{C}$ for 3 hours, warmed at $23{ }^{\circ} \mathrm{C}$ for $30 \mathrm{~min}$, and cooled at $-45^{\circ} \mathrm{C}$ for $30 \mathrm{~min}$. Butadiene (100. $\mu \mathrm{L}, 64.0 \mathrm{mg}, 1.18$ mmol, 1.00 equiv.) and triethoxysilane (218 $\mu \mathrm{L}, 194 \mathrm{mg}, 1.18 \mathrm{mmol}, 1.00$ equiv.) were added. The reaction tube was resealed, removed from the glovebox, and heated to $50{ }^{\circ} \mathrm{C}$ in a pre-heated oil bath for $25 \mathrm{~min}$. before the reaction progress was measured by ${ }^{1} \mathrm{H}$ NMR. By ${ }^{1} \mathrm{H}$ NMR integration of alkenyl signals, $89 \%$ conversion to butenylsilane products was observed. The reaction tube was unsealed and N,N'-dimethylethylenediamine $(10 \mu \mathrm{L})$ was added. The contents of the reaction tube were decanted into a $20 \mathrm{~mL}$ scintillation vial and the solvent was removed by rotary evaporation. The product was purified by column chromatography on silica gel $\left(10 \% \mathrm{v} / \mathrm{v}\right.$ EtOAc/hexanes, $\left.R_{\mathrm{f}}=0.5\right)$ to yield a colorless oil $(0.222 \mathrm{~g}$, $1.02 \mathrm{mmol}, 86 \%$ yield). Ratio of 1,2-:1,4-addition determined to be $10: 1$ by ${ }^{1} \mathrm{H}$ NMR integration of signals corresponding to alkenyl protons (5.96-5.86 ppm for 3-butenyltriethoxysilane signal corresponds to $1 \mathrm{H}$; 5.49-5.37 for 2-butenyltriethoxysilanes, overlapping signals correspond to $2 \mathrm{H}$ ).

\section{Precatalyst 32}

In a dry, $\mathrm{N}_{2}$-filled glovebox, platinum precatalyst $32(3.2 \mathrm{mg}, 5.9 \mu \mathrm{mol}, 0.50 \mathrm{~mol} \%), \mathrm{MgCl}_{2}(\mathrm{THF})_{2}(2.8$ mg, $24 \mu \mathrm{mol}, 2.0 \mathrm{~mol} \%)$, a Teflon-coated magnetic stirring bar, dichloromethane $(0.5 \mathrm{~mL})$, and tetrahydrofuran $(10 \mu \mathrm{L})$ were added to a $4 \mathrm{~mL}$ scintillation vial at $23{ }^{\circ} \mathrm{C}$. The vial was chilled at $-45{ }^{\circ} \mathrm{C}$ in a $\mathrm{CO}_{2} /{ }^{i} \mathrm{PrOH}-$ cooled cold well for $30 \mathrm{~min}$. Butadiene (100. $\mu \mathrm{L}, 64.0 \mathrm{mg}, 1.18 \mathrm{mmol}, 1.00$ equiv.) and triethoxysilane (218 $\mu \mathrm{L}, 194 \mathrm{mg}, 1.18 \mathrm{mmol}, 1.00$ equiv.) were added and the reaction vial was sealed with a Teflon-lined cap, removed from the glovebox, and heated to $50{ }^{\circ} \mathrm{C}$ in a pre-heated aluminum heating block for $25 \mathrm{~min}$. The vial was opened under ambient atmosphere and $\mathrm{TMS}_{2} \mathrm{O}(10 \mu \mathrm{L}, 7.6 \mathrm{mg}, 47$ $\mu \mathrm{mol}, 4.0 \mathrm{~mol} \%)$ and benzene- $d_{6}(100 \mu \mathrm{L})$ were added. The contents of the reaction vial were mixed thoroughly and transferred to an NMR tube. Ratio of 1,2-:1,4-addition determined to be $10: 1$ by ${ }^{1} \mathrm{H}$ NMR 
integration of signals corresponding to alkenyl protons $(5.96-5.86 \mathrm{ppm}$ for 3-butenyltriethoxysilane signal corresponds to $1 \mathrm{H} ; 5.49-5.37$ for 2-butenyltriethoxysilanes, overlapping signals correspond to $2 \mathrm{H}$ ). Yield determined by addition of ${ }^{1} \mathrm{H}$ NMR signal integrations for butenylsilane products (full integration for 3-butenyltriethoxysilane $+1 / 2$ integration for 2-butenyltriethoxysilanes) in comparison to the $\mathrm{TMS}_{2} \mathrm{O}$ signal at $0 \mathrm{ppm}$ (integral set to 71.5 to show integrals as molar percents).

\section{Precatalyst 19}

In a dry, $\mathrm{N}_{2}$-filled glovebox, platinum precatalyst $19(3.9 \mathrm{mg}, 5.9 \mu \mathrm{mol}, 0.5 \mathrm{~mol} \%), \mathrm{MgCl}_{2}(\mathrm{THF})_{2}(5.6$ mg, $24 \mu \mathrm{mol}, 2.0 \mathrm{~mol} \%)$, a Teflon-coated magnetic stirring bar, dichloromethane $(0.5 \mathrm{~mL})$, and tetrahydrofuran $(10 \mu \mathrm{L})$ were added to a $4 \mathrm{~mL}$ scintillation vial at $23{ }^{\circ} \mathrm{C}$. The vial was chilled at $-45{ }^{\circ} \mathrm{C}$ in a $\mathrm{CO}_{2} /{ }^{i} \mathrm{PrOH}$-cooled cold well for $30 \mathrm{~min}$. Butadiene (100. $\mu \mathrm{L}, 64.0 \mathrm{mg}, 1.18 \mathrm{mmol}, 1.00$ equiv.) and triethoxysilane ( $218 \mu \mathrm{L}, 194 \mathrm{mg}, 1.18 \mathrm{mmol}, 1.00$ equiv.) were added and the reaction vial was sealed with a Teflon-lined cap, removed from the glovebox, and heated to $50{ }^{\circ} \mathrm{C}$ in a pre-heated aluminum heating block for $25 \mathrm{~min}$. The reaction vial was opened under ambient atmosphere and $\mathrm{TMS}_{2} \mathrm{O}(10 \mu \mathrm{L}, 47$ $\mu \mathrm{mol}, 4.0 \mathrm{~mol} \%)$ and benzene- $d_{6}(100 \mu \mathrm{L})$ were added. The contents of the reaction vial were mixed thoroughly and transferred to an NMR tube. Ratio of 1,2-:1,4-addition determined to be $9: 1$ by ${ }^{1} \mathrm{H}$ NMR

integration of signals corresponding to alkenyl protons (5.96-5.86 ppm for 3-butenyltriethoxysilane signal corresponds to $1 \mathrm{H} ; 5.49-5.37$ for 2-butenyltriethoxysilanes, overlapping signals correspond to $2 \mathrm{H}$ ). Yield determined as $64 \%$ by addition of ${ }^{1} \mathrm{H}$ NMR signal integrations for butenylsilane products (full integration for 3-butenyltriethoxysilane $+1 / 2$ integration for 2-butenyltriethoxysilanes) in comparison to the $\mathrm{TMS}_{2} \mathrm{O}$ signal at $0 \mathrm{ppm}$ (integral set to 71.5 to show integrals as molar percents).

\section{Precatalyst 19, no additive}

In a dry, $\mathrm{N}_{2}$-filled glovebox, platinum precatalyst $19(3.9 \mathrm{mg}, 5.9 \mu \mathrm{mol}, 0.50 \mathrm{~mol} \%), \mathrm{MgCl}_{2}(\mathrm{THF})_{2}(5.6$ $\mathrm{mg}, 24 \mu \mathrm{mol}, 2.0 \mathrm{~mol} \%$ equiv. $)$, a Teflon-coated magnetic stirring bar, dichloromethane $(0.5 \mathrm{~mL})$, and tetrahydrofuran $(10 \mu \mathrm{L})$ were added to a $4 \mathrm{~mL}$ scintillation vial at $23{ }^{\circ} \mathrm{C}$. The vial was chilled at $-45^{\circ} \mathrm{C}$ in 
a $\mathrm{CO}_{2} /{ }^{i} \mathrm{PrOH}-$ cooled cold well for $30 \mathrm{~min}$. Butadiene (100. $\mu \mathrm{L}, 64.0 \mathrm{mg}, 1.18 \mathrm{mmol}, 1.00$ equiv.) and triethoxysilane (218 $\mu \mathrm{L}, 194 \mathrm{mg}, 1.18 \mathrm{mmol}, 1.00$ equiv.) were added and the reaction vial was sealed with a Teflon-lined cap, removed from the glovebox, and heated to $50{ }^{\circ} \mathrm{C}$ in a pre-heated aluminum heating block for $25 \mathrm{~min}$. The reaction vial was opened under ambient atmosphere and $\mathrm{TMS}_{2} \mathrm{O}(10 \mu \mathrm{L}$, $7.6 \mathrm{mg}, 47 \mu \mathrm{mol}, 4.0 \mathrm{~mol} \%)$ and benzene- $d_{6}(100 \mu \mathrm{L})$ were added. The contents of the reaction vial were mixed thoroughly and transferred to an NMR tube. Ratio of 1,2-:1,4-addition determined to be $9: 1$ by ${ }^{1} \mathrm{H}$ NMR integration of signals corresponding to alkenyl protons (5.96-5.86 ppm for 3-butenyltriethoxysilane signal corresponds to $1 \mathrm{H}$; 5.49-5.37 for 2-butenyltriethoxysilanes, overlapping signals correspond to $2 \mathrm{H}$ ). Yield determined as $10 \%$ by addition of ${ }^{1} \mathrm{H}$ NMR signal integrations for butenylsilane products (full integration for 3-butenyltriethoxysilane $+1 / 2$ integration for 2-butenyltriethoxysilanes) in comparison to the $\mathrm{TMS}_{2} \mathrm{O}$ signal at $0 \mathrm{ppm}$ (integral set to 71.5 to show integrals as molar percents).

\section{Precatalyst 19, 1,4-Dioxane added}

In a dry, $\mathrm{N}_{2}$-filled glovebox, platinum precatalyst $19(3.9 \mathrm{mg}, 5.9 \mu \mathrm{mol}, 0.50 \mathrm{~mol} \%), \mathrm{MgCl}_{2}(\mathrm{THF})_{2}(5.6$ mg, $24 \mu \mathrm{mol}, 2.0 \mathrm{~mol} \%)$, a Teflon-coated magnetic stirring bar, dichloromethane $(0.5 \mathrm{~mL})$, and 1,4dioxane (10 $\mathrm{L}, 10 \mathrm{mg}, 0.12 \mathrm{mmol}, 9.9 \mathrm{~mol} \%)$ were added to a $4 \mathrm{~mL}$ scintillation vial at $23{ }^{\circ} \mathrm{C}$. The reaction vial was chilled at $-45^{\circ} \mathrm{C}$ in a $\mathrm{CO}_{2} / \mathrm{PrOH}-$ cooled cold well for $30 \mathrm{~min}$. Butadiene $(100 . \mu \mathrm{L}, 64.0$ mg, 1.18 mmol, 1.00 equiv.) and triethoxysilane (218 $\mu \mathrm{L}, 194 \mathrm{mg}, 1.18 \mathrm{mmol}, 1.00$ equiv.) were added and the reaction vial was sealed with a Teflon-lined cap, removed from the glovebox, and heated to $50{ }^{\circ} \mathrm{C}$ in a pre-heated aluminum heating block for $25 \mathrm{~min}$. The reaction vial was opened under ambient atmosphere and $\mathrm{TMS}_{2} \mathrm{O}(10 \mu \mathrm{L}, 7.6 \mathrm{mg}, 47 \mu \mathrm{mol}, 4.0 \mathrm{~mol} \%)$ and benzene- $d_{6}(100 \mu \mathrm{L})$ were added. The contents of the reaction vial were mixed thoroughly and transferred to an NMR tube. Ratio of 1,2-:1,4addition determined to be $3: 1$ by ${ }^{1} \mathrm{H}$ NMR integration of signals corresponding to alkenyl protons (5.96$5.86 \mathrm{ppm}$ for 3-butenyltriethoxysilane signal corresponds to $1 \mathrm{H} ; 5.49-5.37$ for 2-butenyltriethoxysilanes, overlapping signals correspond to $2 \mathrm{H}$ ). Yield determined to be $2 \%$ by addition of ${ }^{1} \mathrm{H}$ NMR signal 
integrations for butenylsilane products (full integration for 3-butenyltriethoxysilane $+1 / 2$ integration for 2 butenyltriethoxysilanes) in comparison to the $\mathrm{TMS}_{2} \mathrm{O}$ signal at $0 \mathrm{ppm}$ (integral set to 71.5 to show integrals as molar percents).

\section{Precatalyst 19, Hg added}

In a dry, $\mathrm{N}_{2}$-filled glovebox, platinum precatalyst $19(3.9 \mathrm{mg}, 5.9 \mu \mathrm{mol}, 0.50 \mathrm{~mol} \%), \mathrm{MgCl}_{2}(\mathrm{THF})_{2}(5.6$ mg, $24 \mu \mathrm{mol}, 2.0 \mathrm{~mol} \%)$, a Teflon-coated magnetic stirring bar, dichloromethane $(0.5 \mathrm{~mL})$, and tetrahydrofuran $(10 \mu \mathrm{L})$ were added to a $4 \mathrm{~mL}$ scintillation vial at $23{ }^{\circ} \mathrm{C}$. The vial was chilled to $-45^{\circ} \mathrm{C}$ in a $\mathrm{CO}_{2} /{ }^{i} \mathrm{PrOH}-$ cooled cold well for $30 \mathrm{~min}$. Elemental mercury (5 drops) was added to the reaction vial. Butadiene (100. $\mu \mathrm{L}, 64.0 \mathrm{mg}, 1.18 \mathrm{mmol}, 1.00$ equiv.) and triethoxysilane (218 $\mu \mathrm{L}, 194 \mathrm{mg}, 1.18 \mathrm{mmol}$, 1.00 equiv.) were added and the reaction vial was sealed with a Teflon-lined cap, removed from the glovebox, and heated to $50{ }^{\circ} \mathrm{C}$ in a pre-heated aluminum heating block for $25 \mathrm{~min}$. The reaction vial was opened under ambient atmosphere and $\mathrm{TMS}_{2} \mathrm{O}(10 \mu \mathrm{L}, 7.6 \mathrm{mg}, 47 \mu \mathrm{mol}, 4.0 \mathrm{~mol} \%)$ and benzene- $d_{6}(100$ $\mu \mathrm{L}$ ) were added. The contents of the reaction vial were mixed thoroughly and transferred to an NMR tube. Ratio of 1,2-:1,4-addition determined to be $8: 1$ by ${ }^{1} \mathrm{H}$ NMR integration of signals corresponding to alkenyl protons (5.96-5.86 ppm for 3-butenyltriethoxysilane signal corresponds to $1 \mathrm{H} ; 5.49-5.37$ for 2butenyltriethoxysilanes, overlapping signals correspond to $2 \mathrm{H}$ ). Yield was determined as $52 \%$ by addition of ${ }^{1} \mathrm{H}$ NMR signal integrations for butenylsilane products (full integration for 3-butenyltriethoxysilane + $1 / 2$ integration for 2-butenyltriethoxysilanes) in comparison to the $\mathrm{TMS}_{2} \mathrm{O}$ signal at $0 \mathrm{ppm}$ (integral set to 71.5 to show integrals as molar percents).

\section{Precatalyst 37}

In a dry, $\mathrm{N}_{2}$-filled glovebox, platinum precatalyst 37 (3.9 mg, $\left.5.9 \mu \mathrm{mol}, 0.50 \mathrm{~mol} \%\right), \mathrm{MgCl}_{2}(\mathrm{THF})_{2}(5.6$ mg, $24 \mu \mathrm{mol}, 2.0 \mathrm{~mol} \%)$, a Teflon-coated magnetic stirring bar, dichloromethane $(0.5 \mathrm{~mL})$, and tetrahydrofuran $(10 \mu \mathrm{L})$ were added to a $4 \mathrm{~mL}$ scintillation vial at $23{ }^{\circ} \mathrm{C}$. The vial was chilled at $-45{ }^{\circ} \mathrm{C}$ in a $\mathrm{CO}_{2} /{ }^{i} \mathrm{PrOH}-$ cooled cold well for $30 \mathrm{~min}$. Butadiene (100. $\mu \mathrm{L}, 64.0 \mathrm{mg}, 1.18 \mathrm{mmol}, 1.00$ equiv.) and 
triethoxysilane $(218 \mu \mathrm{L}, 194 \mathrm{mg}, 1.18 \mathrm{mmol}, 1.00$ equiv.) were added and the reaction vial was sealed with a Teflon-lined cap, removed from the glovebox, and heated to $50{ }^{\circ} \mathrm{C}$ in a pre-heated aluminum heating block for $25 \mathrm{~min}$. The reaction vial was opened under ambient atmosphere and $\mathrm{TMS}_{2} \mathrm{O}(10 \mu \mathrm{L}$, $7.6 \mathrm{mg}, 47 \mu \mathrm{mol}, 4.0 \mathrm{~mol} \%)$ and benzene- $d_{6}(100 \mu \mathrm{L})$ were added. The contents of the reaction vial were mixed thoroughly and transferred to an NMR tube. Ratio of 1,2-:1,4-addition determined to be $9: 1$ by ${ }^{1} \mathrm{H}$ NMR integration of signals corresponding to alkenyl protons (5.96-5.86 ppm for 3-butenyltriethoxysilane signal corresponds to $1 \mathrm{H} ; 5.49-5.37$ for 2-butenyltriethoxysilanes, overlapping signals correspond to $2 \mathrm{H}$ ). Yield determined as $28 \%$ by addition of ${ }^{1} \mathrm{H}$ NMR signal integrations for butenylsilane products (full integration for 3-butenyltriethoxysilane $+1 / 2$ integration for 2-butenyltriethoxysilanes) in comparison to the $\mathrm{TMS}_{2} \mathrm{O}$ signal at $0 \mathrm{ppm}$ (integral set to 71.5 to show integrals as molar percents).

\section{Precatalyst 36}

Precatalyst 36 was formed from 42 and butenylmagnesium chloride in $\mathrm{CH}_{2} \mathrm{Cl}_{2}$, observed by ${ }^{1} \mathrm{H}$ and ${ }^{31} \mathrm{P}$ NMR, and used in catalysis without isolation.

\section{Formation of Precatalyst 36}

In a dry, $\mathrm{N}_{2}$-filled glovebox, platinum complex $42(3.6 \mathrm{mg}, 5.9 \mu \mathrm{mol}, 0.50 \mathrm{~mol} \%)$ was added to a $4 \mathrm{~mL}$ scintillation vial and chilled for $30 \mathrm{~min}$. at $-45{ }^{\circ} \mathrm{C}$ in a $\mathrm{CO}_{2} /{ }^{i} \mathrm{PrOH}-$ cooled cold well. Pre-cooled dichloromethane- $d_{2}(0.5 \mathrm{~mL})$ was added to the vial to form a pale yellow solution. But-3-enylmagnesium chloride in THF ( $\mathrm{c}=1.36 \mathrm{M}, 4.4 \mu \mathrm{L}, 5.9 \mu \mathrm{mol}, 0.50 \mathrm{~mol} \%$ ) was added and the vial sealed with a Teflonlined cap and shaken for 90 seconds at $23{ }^{\circ} \mathrm{C}$ to dissolve frozen droplets of Grignard reagent. The solution was stirred for $30 \mathrm{~min}$ at $-45{ }^{\circ} \mathrm{C}$ then transferred to a pre-cooled NMR tube which was sealed with a Teflon-lined cap and removed from the glovebox. ${ }^{1} \mathrm{H}$ and ${ }^{31} \mathrm{P}$ analysis confirmed formation of precatalyst 36. 


\section{Hydrosilylation of Butadiene with Precatalyst $\mathbf{3 6}$}

The NMR tube was brought into a dry, $\mathrm{N}_{2}$-filled glovebox and chilled for $30 \mathrm{~min}$. at $-45{ }^{\circ} \mathrm{C}$ in a $\mathrm{CO}_{2} /{ }^{i} \mathrm{PrOH}$-cooled cold well before butadiene (100. $\mu \mathrm{L}, 64.0 \mathrm{mg}, 1.18 \mathrm{mmol}, 1.00$ equiv.) and triethoxysilane (218 $\mu \mathrm{L}, 194 \mathrm{mg}, 1.18 \mathrm{mmol}, 1.00$ equiv.) were added. The reaction tube was sealed with a Teflon-lined cap, removed from the glovebox, and heated to $50{ }^{\circ} \mathrm{C}$ in a pre-heated oil bath for $25 \mathrm{~min}$. The reaction vial was opened under ambient atmosphere and $\mathrm{TMS}_{2} \mathrm{O}(10 \mu \mathrm{L}, 7.6 \mathrm{mg}, 47 \mu \mathrm{mol}, 4.0 \mathrm{~mol}$ $\%)$ and benzene- $d_{6}(100 \mu \mathrm{L})$ were added. Ratio of 1,2-:1,4-addition determined to be $10: 1$ by ${ }^{1} \mathrm{H}$ NMR integration of signals corresponding to alkenyl protons (5.96-5.86 ppm for 3-butenyltriethoxysilane signal corresponds to $1 \mathrm{H} ; 5.49-5.37$ for 2-butenyltriethoxysilanes, overlapping signals correspond to $2 \mathrm{H}$ ). Yield determined as $65 \%$ by addition of ${ }^{1} \mathrm{H}$ NMR signal integrations for butenylsilane products (full integration for 3-butenyltriethoxysilane $+1 / 2$ integration for 2-butenyltriethoxysilanes) in comparison to the $\mathrm{TMS}_{2} \mathrm{O}$ signal at $0 \mathrm{ppm}$ (integral set to 71.5 to show integrals as molar percents).

\section{Precatalyst 38}

In a dry, $\mathrm{N}_{2}$-filled glovebox, precatalyst $38(4.4 \mathrm{mg}, 6.0 \mu \mathrm{mol}, 0.5 \mathrm{~mol} \%)$, dichloromethane $(0.5 \mathrm{~mL})$, and a Teflon-coated magnetic stirring bar were added to a $4 \mathrm{~mL}$ glass scintillation vial. The vial was cooled at $-45{ }^{\circ} \mathrm{C}$ in a $\mathrm{CO}_{2} /{ }^{i} \mathrm{PrOH}$-cooled cold well for $30 \mathrm{~min}$., then butadiene (100. $\mu \mathrm{L}, 64.0 \mathrm{mg}, 1.18 \mathrm{mmol}, 1.00$ equiv.) and triethoxysilane (218 $\mu \mathrm{L}, 194 \mathrm{mg}, 1.18 \mathrm{mmol}, 1.00$ equiv.) were added and the vial was sealed with a Teflon-lined cap. The vial was removed from the glovebox and heated at $50{ }^{\circ} \mathrm{C}$ in a pre-heated aluminum heating block for $25 \mathrm{~min}$. The vial was opened under ambient atmosphere and $\mathrm{TMS}_{2} \mathrm{O}(10 \mu \mathrm{L})$ and $\mathrm{C}_{6} \mathrm{D}_{6}(100 \mu \mathrm{L})$ were added before the contents were mixed and transferred to an NMR tube. Ratio of 1,2-:1,4-addition determined to be 4:1 by ${ }^{1} \mathrm{H}$ NMR integration of signals corresponding to alkenyl protons (5.96-5.86 ppm for 3-butenyltriethoxysilane signal corresponds to $1 \mathrm{H} ; \quad 5.49-5.37$ for 2butenyltriethoxysilanes, overlapping signals correspond to $2 \mathrm{H}$ ). Yield determined as $11 \%$ by addition of ${ }^{1} \mathrm{H}$ NMR signal integrations for butenylsilane products (full integration for 3-butenyltriethoxysilane $+1 / 2$ 
integration for 2-butenyltriethoxysilanes) in comparison to the $\mathrm{TMS}_{2} \mathrm{O}$ signal at $0 \mathrm{ppm}$ (integral set to 71.5 to show integrals as molar percents).

\section{Precatalyst 39}

In a dry, $\mathrm{N}_{2}$-filled glovebox, precatalyst $39(9.5 \mathrm{mg}, 12 \mu \mathrm{mol}, 1.0 \mathrm{~mol} \%)$, dichloromethane $(0.5 \mathrm{~mL})$, and a Teflon-coated magnetic stirring bar were added to a $4 \mathrm{~mL}$ glass scintillation vial. The vial was cooled at $-45{ }^{\circ} \mathrm{C}$ in a $\mathrm{CO}_{2} /{ }^{i} \mathrm{PrOH}-$ cooled cold well for $30 \mathrm{~min}$., then butadiene (100. $\mu \mathrm{L}, 64.0 \mathrm{mg}, 1.18 \mathrm{mmol}, 1.00$ equiv.) and triethoxysilane ( $218 \mu \mathrm{L}, 194 \mathrm{mg}, 1.18 \mathrm{mmol}, 1.00$ equiv.) were added and the vial was sealed with a Teflon-lined cap. The vial was removed from the glovebox and heated at $50{ }^{\circ} \mathrm{C}$ in a pre-heated aluminum heating block for $25 \mathrm{~min}$. The vial was opened under ambient atmosphere and $\mathrm{TMS}_{2} \mathrm{O}(10 \mu \mathrm{L})$ and $\mathrm{C}_{6} \mathrm{D}_{6}(100 \mu \mathrm{L})$ were added before the contents were mixed and transferred to an NMR tube. Ratio of 1,2-:1,4-addition determined to be $6: 1$ by ${ }^{1} \mathrm{H}$ NMR integration of signals corresponding to alkenyl protons (5.96-5.86 ppm for 3-butenyltriethoxysilane signal corresponds to $1 \mathrm{H} ; \quad 5.49-5.37$ for 2butenyltriethoxysilanes, overlapping signals correspond to $2 \mathrm{H}$ ). Yield determined as $16 \%$ by addition of ${ }^{1} \mathrm{H}$ NMR signal integrations for butenylsilane products (full integration for 3-butenyltriethoxysilane $+1 / 2$ integration for 2-butenyltriethoxysilanes) in comparison to the $\mathrm{TMS}_{2} \mathrm{O}$ signal at $0 \mathrm{ppm}$ (integral set to 71.5 to show integrals as molar percents).

\section{Precatalyst 34}

In a dry, $\mathrm{N}_{2}$-filled glovebox, precatalyst $34(4.5 \mathrm{mg}, 5.9 \mu \mathrm{mol}, 0.50 \mathrm{~mol} \%)$, dichloromethane $(0.5 \mathrm{~mL})$, and a Teflon-coated magnetic stirring bar were added to a $4 \mathrm{~mL}$ glass scintillation vial. The vial was cooled at $-45{ }^{\circ} \mathrm{C}$ in a $\mathrm{CO}_{2} /{ }^{i} \mathrm{PrOH}-$ cooled cold well for $30 \mathrm{~min}$., then butadiene $(100 . \mu \mathrm{L}, 64.0 \mathrm{mg}, 1.18$ mmol, 1.00 equiv.) and triethoxysilane (218 $\mu \mathrm{L}, 194 \mathrm{mg}, 1.18 \mathrm{mmol}, 1.00$ equiv.) were added and the vial was sealed with a Teflon-lined cap. The vial was removed from the glovebox and heated at $50{ }^{\circ} \mathrm{C}$ in a pre-heated aluminum heating block for $25 \mathrm{~min}$. The vial was opened under ambient atmosphere and $\mathrm{TMS}_{2} \mathrm{O}(10 \mu \mathrm{L})$ and $\mathrm{C}_{6} \mathrm{D}_{6}(100 \mu \mathrm{L})$ were added before the contents were mixed and transferred to an 
NMR tube. Yield determined as $<1 \%$ by addition of ${ }^{1} \mathrm{H}$ NMR signal integrations for butenylsilane products (full integration for 3-butenyltriethoxysilane $+1 / 2$ integration for 2 -butenyltriethoxysilanes) in comparison to the $\mathrm{TMS}_{2} \mathrm{O}$ signal at $0 \mathrm{ppm}$ (integral set to 71.5 to show integrals as molar percents).

\section{Precatalyst 34, $\mathrm{MgCl}_{2}$ (THF) 2 Added}

In a dry, $\mathrm{N}_{2}$-filled glovebox, precatalyst $33(11.4 \mathrm{mg}, 13.0 \mu \mathrm{mol}, 1.1 \mathrm{~mol} \%), \mathrm{MgCl}_{2}(\mathrm{THF})_{2}(5.6 \mathrm{mg}, 24$ $\mu \mathrm{mol}, 2.0 \mathrm{~mol} \%)$, dichloromethane $(0.5 \mathrm{~mL})$, and a Teflon-coated magnetic stirring bar were added to a 4 $\mathrm{mL}$ glass scintillation vial. The vial was cooled at $-45^{\circ} \mathrm{C}$ in a $\mathrm{CO}_{2} /{ }^{i} \mathrm{PrOH}-$ cooled cold well for 30 min., then butadiene (100. $\mu \mathrm{L}, 64.0 \mathrm{mg}, 1.18 \mathrm{mmol}, 1.00$ equiv.) and triethoxysilane (218 $\mu \mathrm{L}, 194 \mathrm{mg}, 1.18$ mmol, 1.00 equiv.) were added and the vial was sealed with a Teflon-lined cap. The vial was removed from the glovebox and heated at $50{ }^{\circ} \mathrm{C}$ in a pre-heated aluminum heating block for $25 \mathrm{~min}$. The vial was opened under ambient atmosphere and $\mathrm{TMS}_{2} \mathrm{O}(10 \mu \mathrm{L})$ and $\mathrm{C}_{6} \mathrm{D}_{6}(100 \mu \mathrm{L})$ were added before the contents were mixed and transferred to an NMR tube. Ratio of 1,2-:1,4-addition determined to be 6:1 by ${ }^{1} \mathrm{H}$ NMR integration of signals corresponding to alkenyl protons (5.96-5.86 ppm for 3butenyltriethoxysilane signal corresponds to $1 \mathrm{H} ; 5.49-5.37$ for 2-butenyltriethoxysilanes, overlapping signals correspond to $2 \mathrm{H}$ ). Yield determined as $12 \%$ by addition of ${ }^{1} \mathrm{H}$ NMR signal integrations for butenylsilane products (full integration for 3 -butenyltriethoxysilane $+1 / 2$ integration for 2butenyltriethoxysilanes) in comparison to the $\mathrm{TMS}_{2} \mathrm{O}$ signal at $0 \mathrm{ppm}$ (integral set to 71.5 to show integrals as molar percents).

\section{Precatalyst 34}

In a dry, $\mathrm{N}_{2}$-filled glovebox, precatalyst $34(7.8 \mathrm{mg}, 0.11 \mathrm{mmol}, 9.0 \mathrm{~mol} \%)$, dichloromethane $(0.5 \mathrm{~mL})$, and a Teflon-coated magnetic stirring bar were added to a $4 \mathrm{~mL}$ glass scintillation vial. The vial was cooled at $-45{ }^{\circ} \mathrm{C}$ in a $\mathrm{CO}_{2} /{ }^{i} \mathrm{PrOH}-$ cooled cold well for $30 \mathrm{~min}$., then butadiene (100. $\mu \mathrm{L}, 64.0 \mathrm{mg}, 1.18$ mmol, 1.00 equiv.) and triethoxysilane (218 $\mu \mathrm{L}, 194 \mathrm{mg}, 1.18 \mathrm{mmol}, 1.00$ equiv.) were added and the vial was sealed with a Teflon-lined cap. The vial was removed from the glovebox and heated at $50{ }^{\circ} \mathrm{C}$ in a 
pre-heated aluminum heating block for $25 \mathrm{~min}$. The vial was opened under ambient atmosphere and $\mathrm{TMS}_{2} \mathrm{O}(10 \mu \mathrm{L})$ and $\mathrm{C}_{6} \mathrm{D}_{6}(100 \mu \mathrm{L})$ were added before the contents were mixed and transferred to an NMR tube. Yield determined as $<1 \%$ by addition of ${ }^{1} \mathrm{H}$ NMR signal integrations for butenylsilane products (full integration for 3-butenyltriethoxysilane $+1 / 2$ integration for 2-butenyltriethoxysilanes) in comparison to the $\mathrm{TMS}_{2} \mathrm{O}$ signal at $0 \mathrm{ppm}$ (integral set to 71.5 to show integrals as molar percents).

\section{Precatalyst 33}

In a dry, $\mathrm{N}_{2}$-filled glovebox, precatalyst $33(11.4 \mathrm{mg}, 13.0 \mu \mathrm{mol}, 1.1 \mathrm{~mol} \%)$, dichloromethane $(0.5 \mathrm{~mL})$, and a Teflon-coated magnetic stirring bar were added to a $4 \mathrm{~mL}$ glass scintillation vial. The vial was cooled at $-45{ }^{\circ} \mathrm{C}$ in a $\mathrm{CO}_{2} /{ }^{i} \mathrm{PrOH}$-cooled cold well for $30 \mathrm{~min}$., then butadiene $(100 . \mu \mathrm{L}, 64.0 \mathrm{mg}, 1.18$ mmol, 1.00 equiv.) and triethoxysilane (218 $\mu \mathrm{L}, 194 \mathrm{mg}, 1.18 \mathrm{mmol}, 1.00$ equiv.) were added and the vial was sealed with a Teflon-lined cap. The vial was removed from the glovebox and heated at $50{ }^{\circ} \mathrm{C}$ in a pre-heated aluminum heating block for $25 \mathrm{~min}$. The vial was opened under ambient atmosphere and $\mathrm{TMS}_{2} \mathrm{O}(10 \mu \mathrm{L})$ and $\mathrm{C}_{6} \mathrm{D}_{6}(100 \mu \mathrm{L})$ were added before the contents were mixed and transferred to an NMR tube. Ratio of 1,2-:1,4-addition determined to be $2: 1$ by ${ }^{1} \mathrm{H}$ NMR integration of signals corresponding to alkenyl protons (5.96-5.86 ppm for 3-butenyltriethoxysilane signal corresponds to $1 \mathrm{H}$; 5.49-5.37 for 2-butenyltriethoxysilanes, overlapping signals correspond to $2 \mathrm{H}$ ). Yield determined as 2\% by addition of ${ }^{1} \mathrm{H}$ NMR signal integrations for butenylsilane products (full integration for 3butenyltriethoxysilane $+1 / 2$ integration for 2-butenyltriethoxysilanes) in comparison to the $\mathrm{TMS}_{2} \mathrm{O}$ signal at $0 \mathrm{ppm}$ (integral set to 71.5 to show integrals as molar percents).

\section{Precatalyst 33, $\mathrm{MgCl}_{2}$ (THF)2 Added}

In a dry, $\mathrm{N}_{2}$-filled glovebox, precatalyst $33(11.4 \mathrm{mg}, 13.0 \mu \mathrm{mol}, 1.1 \mathrm{~mol} \%), \mathrm{MgCl}_{2}(\mathrm{THF})_{2}(5.6 \mathrm{mg}, 24$ $\mu \mathrm{mol}, 2.0 \mathrm{~mol} \%)$, dichloromethane $(0.5 \mathrm{~mL})$, and a Teflon-coated magnetic stirring bar were added to a 4 $\mathrm{mL}$ glass scintillation vial. The vial was cooled at $-45^{\circ} \mathrm{C}$ in a $\mathrm{CO}_{2} /{ }^{i} \mathrm{PrOH}-$ cooled cold well for 30 min., then butadiene (100. $\mu \mathrm{L}, 64.0 \mathrm{mg}, 1.18 \mathrm{mmol}, 1.00$ equiv.) and triethoxysilane (218 $\mu \mathrm{L}, 194 \mathrm{mg}, 1.18$ 
mmol, 1.00 equiv.) were added and the vial was sealed with a Teflon-lined cap. The vial was removed from the glovebox and heated at $50{ }^{\circ} \mathrm{C}$ in a pre-heated aluminum heating block for $25 \mathrm{~min}$. The vial was opened under ambient atmosphere and $\mathrm{TMS}_{2} \mathrm{O}(10 \mu \mathrm{L})$ and $\mathrm{C}_{6} \mathrm{D}_{6}(100 \mu \mathrm{L})$ were added before the contents were mixed and transferred to an NMR tube. Ratio of 1,2-:1,4-addition determined to be 7.4:1 by ${ }^{1} \mathrm{H}$ NMR integration of signals corresponding to alkenyl protons (5.96-5.86 ppm for 3butenyltriethoxysilane signal corresponds to $1 \mathrm{H} ; 5.49-5.37$ for 2-butenyltriethoxysilanes, overlapping signals correspond to $2 \mathrm{H}$ ). Yield determined as $30 \%$ by addition of ${ }^{1} \mathrm{H}$ NMR signal integrations for butenylsilane products (full integration for 3-butenyltriethoxysilane $+1 / 2$ integration for 2butenyltriethoxysilanes) in comparison to the $\mathrm{TMS}_{2} \mathrm{O}$ signal at $0 \mathrm{ppm}$ (integral set to 71.5 to show integrals as molar percents).

\section{Karstedt's Catalyst (2)}

In a dry, $\mathrm{N}_{2}$-filled glovebox, Karstedt's catalyst $(37.8 \mathrm{mg}, 3 \mathrm{w} / \mathrm{w} \% \mathrm{Pt}$ in vinyl-terminated polydimethylsiloxane, $5.90 \mu \mathrm{mol}, 0.500 \mathrm{~mol} \%$ ), a Teflon-coated magnetic stirring bar, dichloromethane $(0.5 \mathrm{~mL})$, and tetrahydrofuran $(10 \mu \mathrm{L})$ were added to a $4 \mathrm{~mL}$ scintillation vial at $23{ }^{\circ} \mathrm{C}$. The vial was chilled at $-45{ }^{\circ} \mathrm{C}$ in a $\mathrm{CO}_{2} /{ }^{i} \mathrm{PrOH}-$ cooled cold well for $30 \mathrm{~min}$. Butadiene (100. $\mu \mathrm{L}, 64.0 \mathrm{mg}, 1.18 \mathrm{mmol}$, 1.00 equiv.) and triethoxysilane (218 $\mu \mathrm{L}, 194 \mathrm{mg}, 1.18 \mathrm{mmol}, 1.00$ equiv.) were added and the reaction vial was sealed with a Teflon-lined cap, removed from the glovebox, and heated to $50{ }^{\circ} \mathrm{C}$ in a pre-heated aluminum heating block for $25 \mathrm{~min}$. The reaction vial was opened under ambient atmosphere and $\mathrm{TMS}_{2} \mathrm{O}$ (10 $\mu \mathrm{L}, 7.6 \mathrm{mg}, 47 \mu \mathrm{mol}, 4.0 \mathrm{~mol} \%)$ and benzene- $d_{6}(100 \mu \mathrm{L})$ were added. The contents of the reaction vial were mixed thoroughly and transferred to an NMR tube. Ratio of 1,2-:1,4-addition determined to be 1:10 by ${ }^{1} \mathrm{H}$ NMR integration of signals corresponding to alkenyl protons (5.96-5.86 ppm for 3butenyltriethoxysilane signal corresponds to $1 \mathrm{H} ; 5.49-5.37$ for 2-butenyltriethoxysilanes, overlapping signals correspond to $2 \mathrm{H}$ ). Yield determined as $10 \%$ by addition of ${ }^{1} \mathrm{H}$ NMR signal integrations for butenylsilane products (full integration for 3-butenyltriethoxysilane $+1 / 2$ integration for 2- 
butenyltriethoxysilanes) in comparison to the $\mathrm{TMS}_{2} \mathrm{O}$ signal at $0 \mathrm{ppm}$ (integral set to 71.5 to show integrals as molar percents).

\section{Karstedt's Catalyst (2), $\mathrm{P}\left({ }^{t} \mathrm{Bu}\right)_{3}$ added}

In a dry, $\mathrm{N}_{2}$-filled glovebox, Karstedt's Catalyst $(37.8 \mathrm{mg}, 3 \mathrm{w} / \mathrm{w} \% \mathrm{Pt}$ in vinyl-terminated siloxane polymer, $5.9 \mu \mathrm{mol}, 0.500 \mathrm{~mol} \%), \mathrm{P}\left({ }^{t} \mathrm{Bu}\right)_{3}(1.2 \mathrm{mg}, 5.9 \mu \mathrm{mol}, 0.50 \mathrm{~mol} \%)$, a Teflon-coated magnetic stirring bar, dichloromethane $(0.5 \mathrm{~mL})$, and tetrahydrofuran $(10 \mu \mathrm{L})$ were added to a $4 \mathrm{~mL}$ scintillation vial at $23{ }^{\circ} \mathrm{C}$. The vial was chilled at $-45^{\circ} \mathrm{C}$ in a $\mathrm{CO}_{2} /{ }^{i} \mathrm{PrOH}$-cooled cold well for $30 \mathrm{~min}$. Butadiene (100. $\mu \mathrm{L}, 64.0 \mathrm{mg}, 1.18 \mathrm{mmol}, 1.00$ equiv.) and triethoxysilane (218 $\mu \mathrm{L}, 194 \mathrm{mg}, 1.18 \mathrm{mmol}, 1.00$ equiv.) were added and the reaction vial was sealed with a Teflon-lined cap, removed from the glovebox, and heated to $50{ }^{\circ} \mathrm{C}$ in a pre-heated aluminum heating block for $25 \mathrm{~min}$. The reaction vial was opened under ambient atmosphere and $\mathrm{TMS}_{2} \mathrm{O}(10 \mu \mathrm{L}, 7.6 \mathrm{mg}, 47 \mu \mathrm{mol}, 4.0 \mathrm{~mol} \%)$ and benzene- $d_{6}(100 \mu \mathrm{L})$ were added. The contents of the reaction vial were mixed thoroughly and transferred to an NMR tube. Ratio of 1,2-:1,4addition determined to be $1: 8$ by ${ }^{1} \mathrm{H}$ NMR integration of signals corresponding to alkenyl protons (5.96$5.86 \mathrm{ppm}$ for 3-butenyltriethoxysilane signal corresponds to $1 \mathrm{H} ; 5.49-5.37$ for 2-butenyltriethoxysilanes, overlapping signals correspond to $2 \mathrm{H})$. Yield determined as $3 \%$ by addition of ${ }^{1} \mathrm{H}$ NMR signal integrations for butenylsilane products (full integration for 3-butenyltriethoxysilane $+1 / 2$ integration for 2butenyltriethoxysilanes) in comparison to the $\mathrm{TMS}_{2} \mathrm{O}$ signal at $0 \mathrm{ppm}$ (integral set to 71.5 to show integrals as molar percents).

\subsubsection{Isomerization of 3-Butenylsilane to 2-Butenylsilane}

After the hydrosilylation of butadiene using precatalyst $\mathbf{1 6}$ reaches full conversion, any excess of triethoxysilane induces isomerization of the desired 1,2-addition product (3-butenyltriethoxysilane) to the thermodynamically favored 1,4-addition product (2-cis- or 2-trans-butenyltriethoxysilane). At a 1:1 ratio of silane to diene, the volatile diene is always slightly depleted compared to the quantity of silane in the reaction mixture, which leads to rapid product isomerization. To demonstrate the isomerization of 3 - 
butenylsilane to 2-butenylsilane in situ, we recorded a timecourse for hydrosilylation using precatalyst $\mathbf{1 6}$ in which we allowed the reaction to run past full conversion.

\section{Procedure}

In a dry, $\mathrm{N}_{2}$-filled glovebox, platinum precatalyst $16(6.4 \mathrm{mg}, 7.4 \mu \mathrm{mol}, 0.63 \mathrm{~mol} \%)$, a Teflon-coated magnetic stirring bar, and PhMe- $d^{8}(0.5 \mathrm{~mL})$ were added to a screw-capped NMR tube. After chilling at $-45^{\circ} \mathrm{C}$ in a $\mathrm{CO}_{2} / \mathrm{PrOH}-$ cooled cold well for 30 min., methylmagnesium chloride in THF was added $(\mathrm{c}=$ $3.3 \mathrm{M}, 10 \mu \mathrm{L}, 30 \mu \mathrm{mol}, 2.6 \mathrm{~mol} \%$ ) and the vial was sealed with a Teflon-lined cap and shaken for 90 seconds at $23{ }^{\circ} \mathrm{C}$ to dissolve frozen droplets of Grignard reagent. The reaction mixture was chilled at -45 ${ }^{\circ} \mathrm{C}$ for 3 hours then warmed at $23{ }^{\circ} \mathrm{C}$ for $30 \mathrm{~min}$. The tube was chilled at $-45{ }^{\circ} \mathrm{C}$ for $30 \mathrm{~min}$. then butadiene (100. $\mu \mathrm{L}, 64.0 \mathrm{mg}, 1.18 \mathrm{mmol}, 1.00$ equiv.) and triethoxysilane (218 $\mu \mathrm{L}, 194 \mathrm{mg}, 1.18 \mathrm{mmol}$, 1.00 equiv.) were added. The NMR tube was sealed with a Teflon-lined cap, removed from the glovebox, and inserted into a pre-heated $400 \mathrm{MHz}$ NMR Spectrometer at $50{ }^{\circ} \mathrm{C} .{ }^{1} \mathrm{H}$ NMR spectra were measured periodically for $90 \mathrm{~min}$.

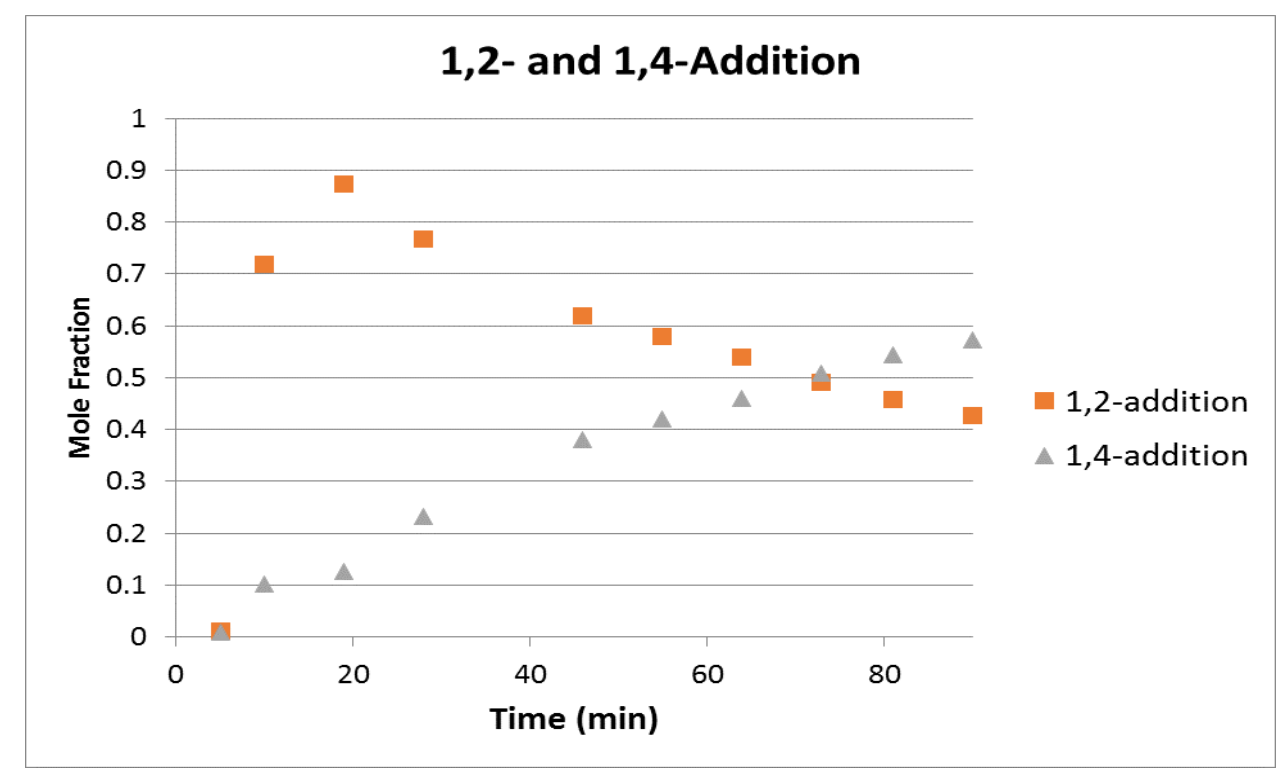

Figure 12. Isomerization of 3-Butenylsilane Using Precatalyst 16 


\subsubsection{Rate Comparison of Precatalysts $\mathbf{3 2}$ and $\mathbf{3 7}$}

To support our claim that precatalyst $\mathbf{3 2}$ exhibits a faster rate than precatalyst $\mathbf{3 7}$ in the hydrosilylation of butadiene, we measured the conversion vs. time using the two precatalysts under identical conditions.

\section{Precatalyst 32}

In a dry, $\mathrm{N}_{2}$-filled glovebox, platinum precatalyst $32(6.5 \mathrm{mg}, 12 \mu \mathrm{mol}, 1.0 \mathrm{~mol} \%), \mathrm{MgCl}_{2}\left(\mathrm{THF}_{2}(5.6\right.$ mg, $24 \mu \mathrm{mol}, 2.0 \mathrm{~mol} \%)$, dichloromethane- $d_{2}(0.5 \mathrm{~mL})$, tetrahydrofuran $(10 \mu \mathrm{L})$, and hexamethyldisiloxane (internal standard, $10 \mu \mathrm{L}, 7.6 \mathrm{mg}, 47 \mu \mathrm{mol}, 4.0 \mathrm{~mol} \%$ ) were added to a $4 \mathrm{~mL}$ scintillation vial at $23{ }^{\circ} \mathrm{C}$. The vial was chilled at $-45{ }^{\circ} \mathrm{C}$ in a $\mathrm{CO}_{2} / \mathrm{PrOH}$-cooled cold well for $30 \mathrm{~min}$. Butadiene (120. $\mu \mathrm{L}, 77.0 \mathrm{mg}, 1.42 \mathrm{mmol}, 1.20$ equiv.) and triethoxysilane (218 $\mu \mathrm{L}, 194 \mathrm{mg}, 1.18 \mathrm{mmol}$, 1.00 equiv.) were added and the reaction mixture was transferred to a pre-cooled J. Young NMR Tube and sealed with a Teflon cap. The tube was removed from the glovebox and cooled at $0{ }^{\circ} \mathrm{C}$ for approximately 5 minutes before inserting into a $600 \mathrm{MHz}$ NMR spectrometer pre-heated to $50{ }^{\circ} \mathrm{C}$. Insertion time corresponds to time $=0$ for kinetic measurements. ${ }^{1} \mathrm{H}$ NMR spectra were collected approximately each minute for 1 hour, at which point no triethoxysilane was observed in the ${ }^{1} \mathrm{H}$ NMR spectrum of the reaction. Integration of ${ }^{1} \mathrm{H}$ NMR signals in the alkenyl region (5.96-5.86 ppm for 3butenyltriethoxysilane signal corresponds to $1 \mathrm{H} ; 5.49-5.37$ for 2-butenyltriethoxysilanes, overlapping signals correspond to $2 \mathrm{H}$ ) were used to determine yield of 1,2- and 1,4-addition products, in comparison to the integration of the signal at $0 \mathrm{ppm}$ corresponding to $\mathrm{TMS}_{2} \mathrm{O}$. 


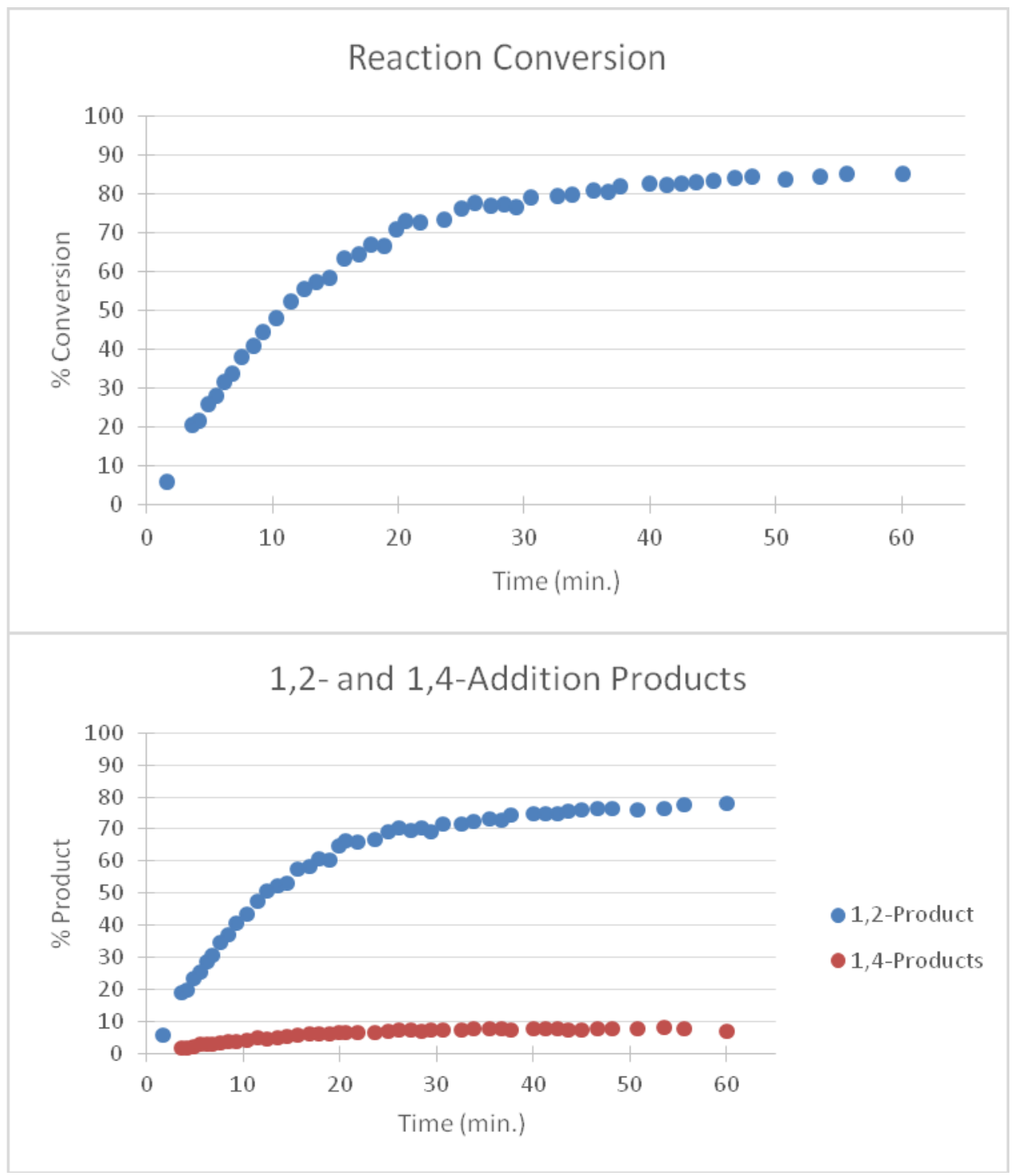

Figure 13. Hydrosilylation Timecourse Using Precatalyst 32

\section{Precatalyst 37}

In a dry, $\mathrm{N}_{2}$-filled glovebox, platinum precatalyst 37 (7.2 mg, $\left.12 \mu \mathrm{mol}, 1.0 \mathrm{~mol} \%\right), \mathrm{MgCl}_{2}(\mathrm{THF})_{2}$ (5.8 $\mathrm{mg}, 24 \mu \mathrm{mol}, 2.0 \mathrm{~mol} \%)$, dichloromethane- $d_{2}(0.5 \mathrm{~mL})$, and tetrahydrofuran $(10 \mu \mathrm{L})$ were added to a $4 \mathrm{~mL}$ scintillation vial at $23{ }^{\circ} \mathrm{C}$. The vial was cooled at $-45{ }^{\circ} \mathrm{C}$ in a $\mathrm{CO}_{2} /{ }^{i} \mathrm{PrOH}$-cooled cold well for 30 min. Butadiene (120. $\mu \mathrm{L}, 77.0 \mathrm{mg}, 1.42 \mathrm{mmol}, 1.20$ equiv.), triethoxysilane (218 $\mu \mathrm{L}, 194 \mathrm{mg}, 1.18 \mathrm{mmol}$, 1.00 equiv.) and hexamethyldisiloxane (internal standard, $10 \mu \mathrm{L}, 7.6 \mathrm{mg}, 47 \mu \mathrm{mol}, 4.0 \mathrm{~mol} \%$ ) were 
added and the reaction mixture was transferred to a pre-cooled NMR Tube that was sealed with a Teflonlined cap. The reaction tube was removed from the glovebox and inserted into a $600 \mathrm{MHz}$ NMR spectrometer pre-heated to $50{ }^{\circ} \mathrm{C}$. Insertion time corresponds to time $=0$ for kinetic measurements. ${ }^{1} \mathrm{H}$ NMR spectra were collected periodically for 16 hours, at which point $>90 \%$ conversion was observed in the ${ }^{1} \mathrm{H}$ NMR spectrum of the reaction. Integration of ${ }^{1} \mathrm{H}$ NMR signals in the alkenyl region (5.94-5.88 ppm for 3-butenyltriethoxysilane signal corresponds to $1 \mathrm{H}$; 5.48-5.38 for 2-butenyltriethoxysilanes, overlapping signals correspond to $2 \mathrm{H}$ ) were used to determine yield of 1,2- and 1,4-addition products, in comparison to the integration of the signal at $0 \mathrm{ppm}$ corresponding to $\mathrm{TMS}_{2} \mathrm{O}$. 


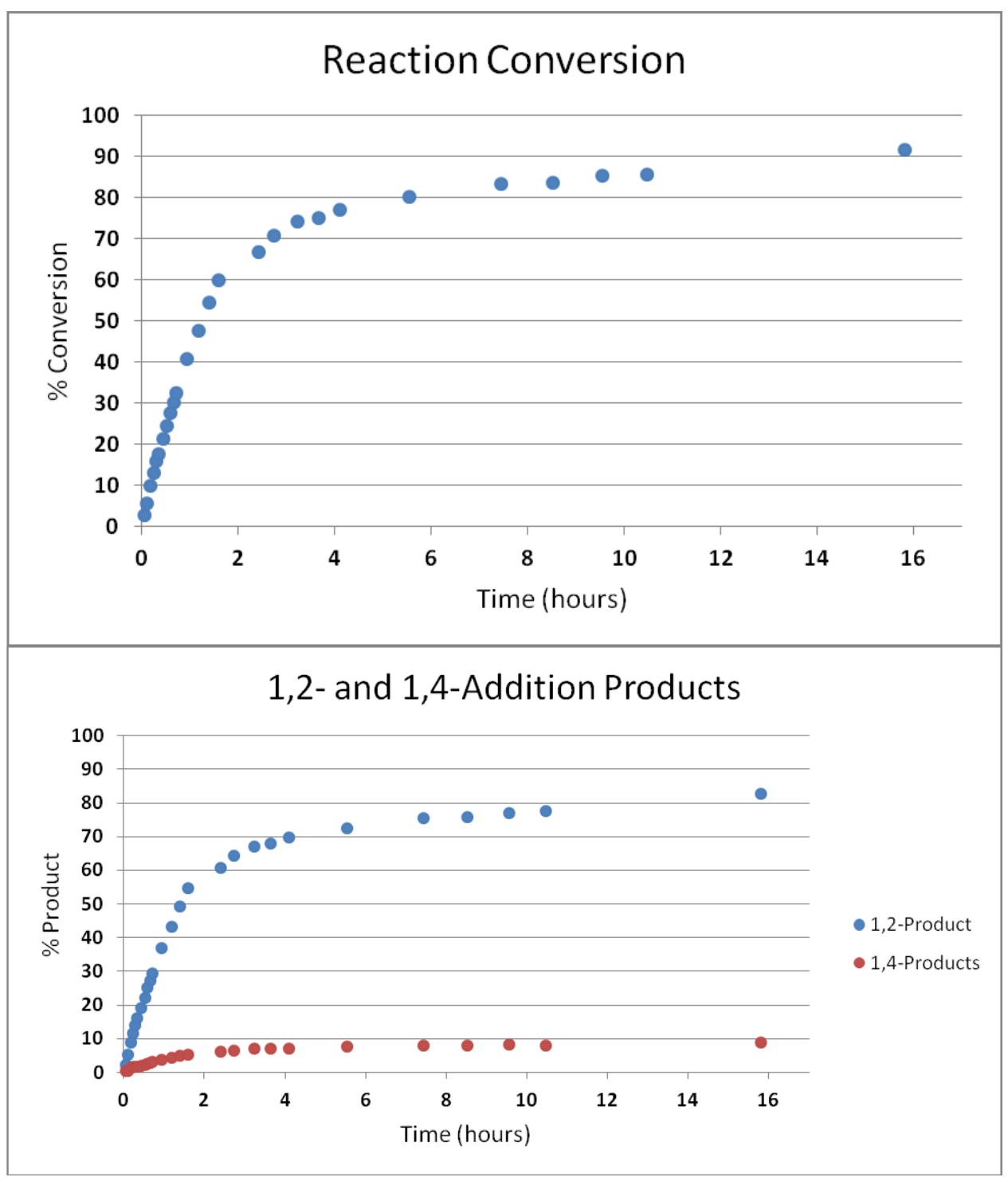

Figure 14. Hydrosilylation Timecourse Using Precatalyst 37

4.6.8. Reaction Timecourse for Hydrosilylation Catalyzed by 16, 19 and 36

\section{Precatalyst 16}

In a dry, $\mathrm{N}_{2}$-filled glovebox, platinum precatalyst $16(3.3 \mathrm{mg}, 3.8 \mu \mathrm{mol}, 0.33 \mathrm{~mol} \%)$, a Teflon-coated magnetic stirring bar, and PhMe- $d^{8}(0.5 \mathrm{~mL})$ were added to a screw-capped NMR tube. After chilling at $-45{ }^{\circ} \mathrm{C}$ in a $\mathrm{CO}_{2} /{ }^{i} \mathrm{PrOH}$-cooled cold well for $30 \mathrm{~min}$., methylmagnesium chloride in THF was added $(\mathrm{c}=$ 3.0 M, $4.6 \mu \mathrm{L}, 15 \mu \mathrm{mol}, 1.3 \mathrm{~mol} \%$ ) and the vial was sealed with a Teflon-lined cap and shaken for 90 
seconds at $23{ }^{\circ} \mathrm{C}$ to dissolve frozen droplets of Grignard reagent. The reaction mixture was chilled at -45 ${ }^{\circ} \mathrm{C}$ for 3 hours then warmed at $23{ }^{\circ} \mathrm{C}$ for $30 \mathrm{~min}$. The tube was chilled at $-45{ }^{\circ} \mathrm{C}$ for $30 \mathrm{~min}$. then butadiene (100. $\mu \mathrm{L}, 64.0 \mathrm{mg}, 1.18 \mathrm{mmol}, 1.00$ equiv.) and triethoxysilane (218. $\mu \mathrm{L}, 194 \mathrm{mg}, 1.18 \mathrm{mmol}$, 1.00 equiv.) were added. The NMR tube was sealed with a Teflon-lined cap, removed from the glovebox, and inserted into a pre-heated $400 \mathrm{MHz}$ NMR Spectrometer at $50{ }^{\circ} \mathrm{C} .{ }^{1} \mathrm{H}$ NMR spectra were measured periodically for $90 \mathrm{~min}$. until the reaction reached full conversion.

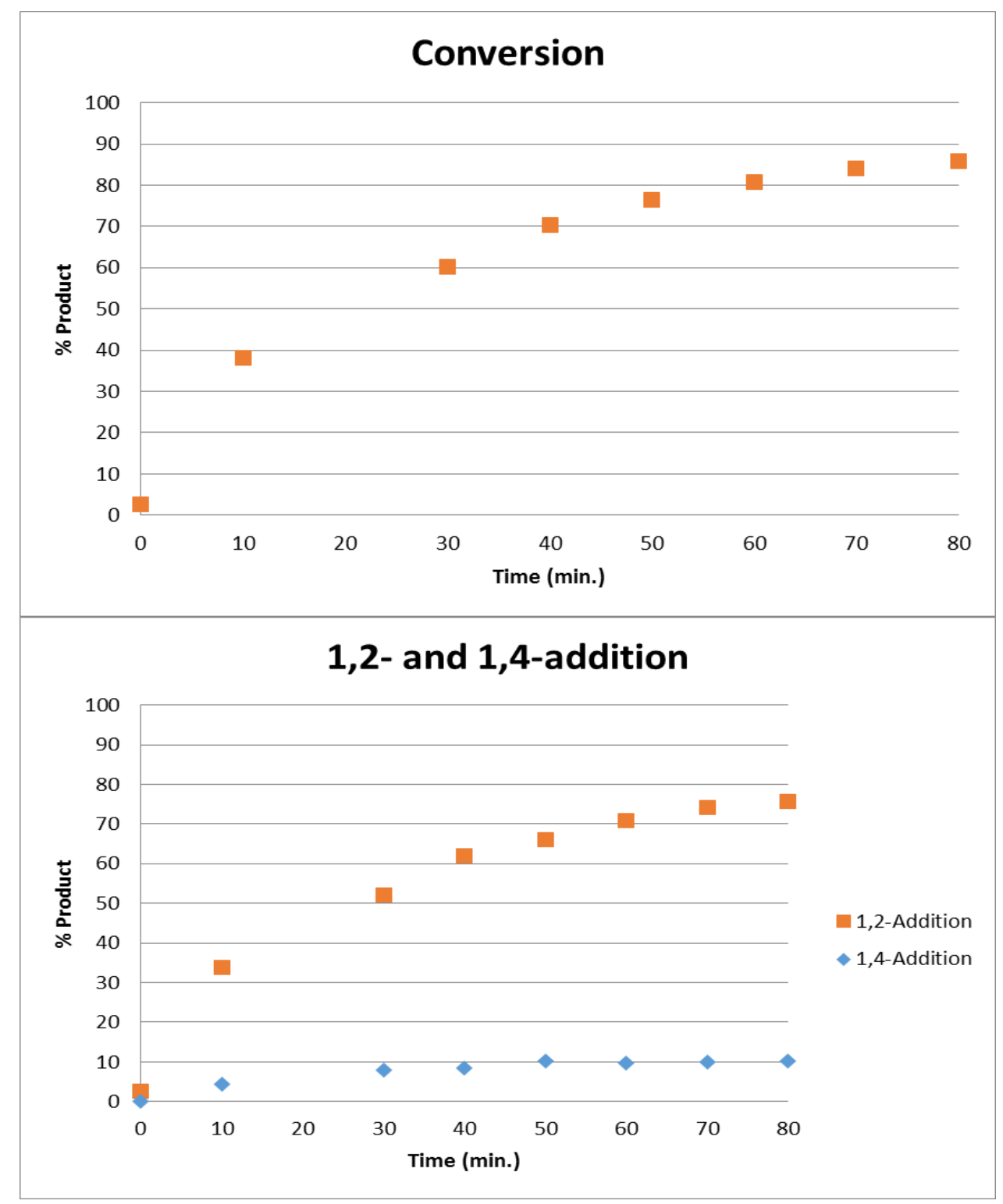

Figure 15. Hydrosilylation Timecourse Using Precatalyst 16 


\section{Precatalyst 19}

In a dry, $\mathrm{N}_{2}$-filled glovebox, platinum precatalyst $19(3.9 \mathrm{mg}, 5.9 \mu \mathrm{mol}, 0.5 \mathrm{~mol} \%), \mathrm{MgCl}_{2}(\mathrm{THF})_{2}(2.8$ mg, $12 \mu \mathrm{mol}, \quad 1.0 \mathrm{~mol} \%)$, dichloromethane- $d_{2}(0.5 \mathrm{~mL})$, tetrahydrofuran $(10 \mu \mathrm{L})$, and hexamethyldisiloxane (internal standard, $10 \mu \mathrm{L}, 7.6 \mathrm{mg}, 47 \mu \mathrm{mol}, 4.0 \mathrm{~mol} \%$ ) were added to a $4 \mathrm{~mL}$ scintillation vial at $23{ }^{\circ} \mathrm{C}$. The vial was chilled at $-45{ }^{\circ} \mathrm{C}$ in a $\mathrm{CO}_{2} /{ }^{i} \mathrm{PrOH}$-cooled cold well for 30 min. Butadiene (150. $\mu \mathrm{L}, 96.0 \mathrm{mg}, 1.77 \mathrm{mmol}, 1.50$ equiv.) and triethoxysilane (218 $\mu \mathrm{L}, 194 \mathrm{mg}, 1.18 \mathrm{mmol}$, 1.00 equiv.) were added and the reaction mixture was transferred to a pre-cooled screw-capped NMR Tube and sealed with a Teflon-lined cap. The tube was removed from the glovebox and warmed at $23{ }^{\circ} \mathrm{C}$ for approximately 5 minutes before inserting into a $500 \mathrm{MHz}$ NMR spectrometer pre-heated to $50{ }^{\circ} \mathrm{C}$. Insertion time corresponds to time $=0$ for kinetic measurements. ${ }^{1} \mathrm{H}$ NMR spectra were collected periodically for 1.5 hours, at which point no triethoxysilane was observed in the ${ }^{1} \mathrm{H}$ NMR spectrum of the reaction. Integration of ${ }^{1} \mathrm{H}$ NMR signals in the alkenyl region (5.96-5.86 ppm for 3butenyltriethoxysilane signal corresponds to $1 \mathrm{H} ; 5.49-5.37$ for 2-butenyltriethoxysilanes, overlapping signals correspond to $2 \mathrm{H}$ ) were used to determine yield of 1,2- and 1,4-addition products, in comparison to the integration of the signal at 0 ppm corresponding to $\mathrm{TMS}_{2} \mathrm{O}$. 


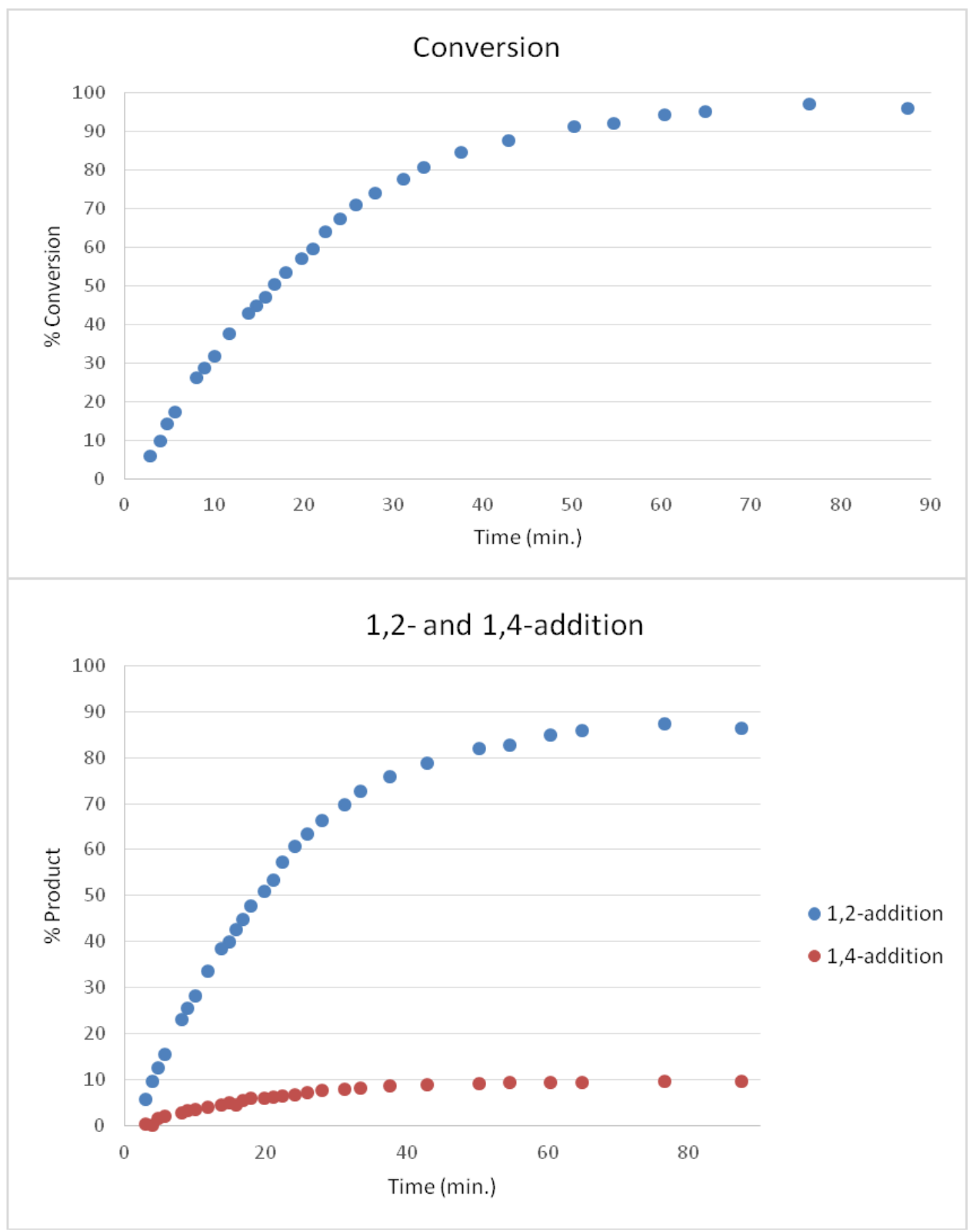

Figure 16. Hydrosilylation Timecourse Using Precatalyst 19

\section{Precatalyst 36}

In a dry, $\mathrm{N}_{2}$-filled glovebox, platinum complex $42(3.6 \mathrm{mg}, 5.9 \mu \mathrm{mol}, 0.50 \mathrm{~mol} \%)$ was added to a $4 \mathrm{~mL}$ scintillation vial and chilled for $30 \mathrm{~min}$. at $-45{ }^{\circ} \mathrm{C}$ in a $\mathrm{CO}_{2} /{ }^{i} \mathrm{PrOH}-$ cooled cold well. Pre-cooled dichloromethane- $d_{2}(0.5 \mathrm{~mL})$ was added to the vial to form a pale yellow solution. But-3-enylmagnesium chloride in THF ( $\mathrm{c}=1.36 \mathrm{M}, 4.4 \mu \mathrm{L}, 5.9 \mu \mathrm{mol}, 0.50 \mathrm{~mol} \%$ ) was added and the vial sealed with a Teflon- 
lined cap and shaken for 90 seconds at $23{ }^{\circ} \mathrm{C}$ to dissolve frozen droplets of Grignard reagent. The solution was stirred for $15 \mathrm{~min}$ at $-45{ }^{\circ} \mathrm{C}$ then warmed at $23{ }^{\circ} \mathrm{C}$ for $15 \mathrm{~min}$ and cooled at $-45{ }^{\circ} \mathrm{C}$ for $30 \mathrm{~min}$. Butadiene (150. $\mu \mathrm{L}, 96.0 \mathrm{mg}, 1.77 \mathrm{mmol}, 1.50$ equiv.), triethoxysilane (218 $\mu \mathrm{L}, 194 \mathrm{mg}, 1.18 \mathrm{mmol}, 1.00$ equiv.), and $\mathrm{TMS}_{2} \mathrm{O}$ (internal standard, $10 \mu \mathrm{L}, 7.6 \mathrm{mg}, 47 \mu \mathrm{mol}, 4.0 \mathrm{~mol} \%$ ) were added. The reaction mixture was transferred to a pre-cooled screw-capped NMR Tube and sealed with a Teflon-lined cap. The tube was removed from the glovebox and warmed at $23{ }^{\circ} \mathrm{C}$ for approximately 5 minutes before inserting into a $500 \mathrm{MHz}$ NMR spectrometer pre-heated to $50{ }^{\circ} \mathrm{C}$. Insertion time corresponds to time $=0$ for kinetic measurements. ${ }^{1} \mathrm{H}$ NMR spectra were collected periodically for 2 hours, at which point no triethoxysilane was observed in the ${ }^{1} \mathrm{H}$ NMR spectrum of the reaction. Integration of ${ }^{1} \mathrm{H}$ NMR signals in the alkenyl region (5.96-5.86 ppm for 3-butenyltriethoxysilane signal corresponds to $1 \mathrm{H} ; 5.49-5.37$ for 2butenyltriethoxysilanes, overlapping signals correspond to $2 \mathrm{H}$ ) were used to determine yield of 1,2- and 1,4-addition products, in comparison to the integration of the signal at $0 \mathrm{ppm}$ corresponding to $\mathrm{TMS}_{2} \mathrm{O}$. 


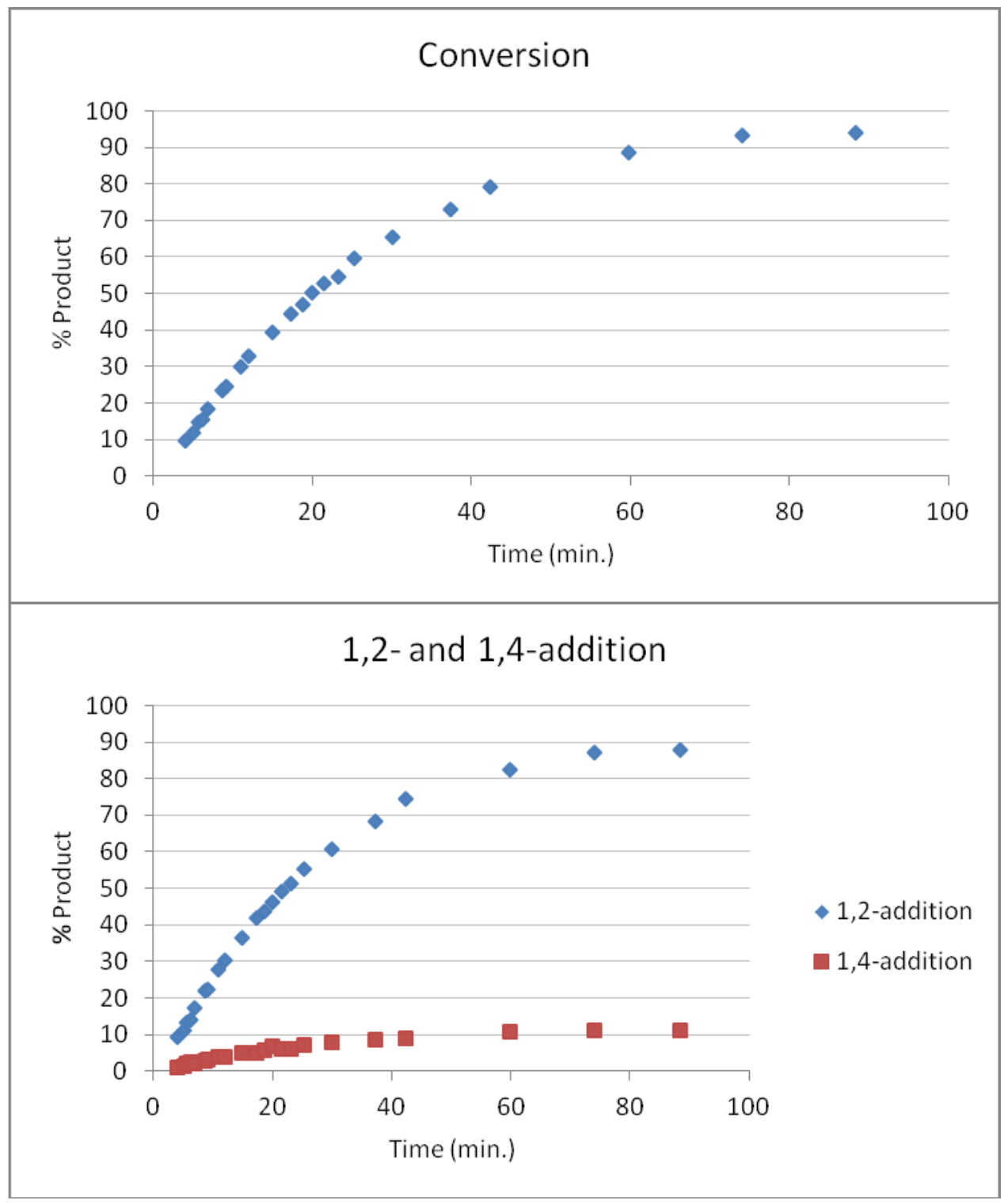

Figure 17. Hydrosilylation Timecourse Using Precatalyst 36 